\title{
Clinical outcomes after innovative lamellar corneal transplantation surgery
}

Citation for published version (APA):

Cheng, Y. Y. Y. (2015). Clinical outcomes after innovative lamellar corneal transplantation surgery.

[Doctoral Thesis, Maastricht University]. Maastricht University. https://doi.org/10.26481/dis.20151221yc

Document status and date:

Published: 01/01/2015

DOI:

10.26481/dis.20151221yc

Document Version:

Publisher's PDF, also known as Version of record

\section{Please check the document version of this publication:}

- A submitted manuscript is the version of the article upon submission and before peer-review. There can be important differences between the submitted version and the official published version of record.

People interested in the research are advised to contact the author for the final version of the publication, or visit the DOI to the publisher's website.

- The final author version and the galley proof are versions of the publication after peer review.

- The final published version features the final layout of the paper including the volume, issue and page numbers.

Link to publication

\footnotetext{
General rights rights.

- You may freely distribute the URL identifying the publication in the public portal. please follow below link for the End User Agreement:

www.umlib.nl/taverne-license

Take down policy

If you believe that this document breaches copyright please contact us at:

repository@maastrichtuniversity.nl

providing details and we will investigate your claim.
}

Copyright and moral rights for the publications made accessible in the public portal are retained by the authors and/or other copyright owners and it is a condition of accessing publications that users recognise and abide by the legal requirements associated with these

- Users may download and print one copy of any publication from the public portal for the purpose of private study or research.

- You may not further distribute the material or use it for any profit-making activity or commercial gain

If the publication is distributed under the terms of Article $25 \mathrm{fa}$ of the Dutch Copyright Act, indicated by the "Taverne" license above, 
Clinical Outcomes After Innovative Lamellar Corneal Transplantation Surgery 
Clinical Outcomes After Innovative Lamellar Corneal Transplantation Surgery Yanny Y.Y. Cheng

ISBN: 978-94-6169-759-2

Printing and lay out: Optima grafische communicatie Rotterdam, the Netherlands Cover photograph: scanning electron microscopy of the corneal stroma Illustrations chapter 1: Rogier Trompert Medical art Illustrations chapter 2 and 6: Geertjan van Zonneveld

Additional financial support for this thesis was kindly provided by: University Eye Clinic Maastricht from the Medical University Center Maastricht (c) 2015, Yanny Y.Y. Cheng

All right reserved. No part of this thesis may be reproduced or transmitted in any form or by any means, electronic or mechanical, including Photocopying, recording of any information storage or retrieval system, without permission in writing from the author, or when appropriate, from the publishers or the publications. 


\title{
Clinical Outcomes After Innovative Lamellar Corneal Transplantation Surgery
}

\author{
PROEFSCHRIFT
}

ter verkrijging van de graad van doctor aan de Universiteit Maastricht, op gezag van de Rector Magnificus, Prof. dr. L.L.G. Soete, volgens het besluit van het College van Decanen, in het openbaar te verdedigen op maandag 21 december 2015 om 14.00 uur

door

Yanny Ying-Yee Cheng 


\section{Promotor}

Prof. dr. R.M.M.A. Nuijts

\section{Copromotor}

Dr. J.S.A.G. Schouten

\section{Beoordelingscommissie}

Prof. dr. H.W.M. Steinbusch (voorzitter)

Prof. dr. S.M. Imhof (Universitair Medisch Centrum Utrecht)

Prof. dr. G.P.M. Luyten (Leids Universitair Medisch Centrum)

Prof. dr. P.M. Steijlen

Prof. dr. M.G.J. Tilanus

The studies in this thesis are supported by a grant from "ZonMw - The Netherlands Organisation for Health Research and Development", "Rotterdamse Vereniging Blindenbelangen", "Algemene Nederlandse Vereniging ter Voorkoming van Blindheid", "board of directors of Maastricht University Medical Center, "Landelijke Stichting voor Blinden en Slechtzienden", and "Stichting Blindenhulp". 
Voor mijn ouders 



\section{CONTENTS}

List of Abbreviations

$\begin{array}{lll}\text { Chapter } 1 \text { General introduction } & 13\end{array}$

Part $1 \quad$ Femtosecond laser-assisted keratoplasty

Chapter 2 Histologic evaluation of human posterior lamellar discs 35

for femtosecond laser Descemet's stripping endothelial

keratoplasty

Cornea. 2009 Jan;28(1):73-79

Chapter 3 Corneal endothelial viability after femtosecond laser

preparation of posterior lamellar discs for Descemet-stripping

endothelial keratoplasty

Cornea. 2007 Oct;26(9):1118-22

Chapter 4 Femtosecond-laser-assisted Descemet's stripping endothelial keratoplasty

J Cataract Refract Surg. 2007 Jan;33(1):152-55

Chapter 5 Preliminary results of femtosecond laser-assisted Descemet

stripping endothelial keratoplasty

Arch Ophthalmol. 2008 Oct;126(10):1351-56

Chapter 6 Efficacy and safety of femtosecond laser-assisted corneal

endothelial keratoplasty: a randomized multicenter clinical trial Transplantation. 2009 Dec 15;88(11):1294-302

Chapter 7 Quality of vision after femtosecond laser-assisted Descemet stripping endothelial keratoplasty and penetrating keratoplasty: a randomized, multicenter clinical trial Am J Ophthalmol. 2011 Oct;152(4):556-66

Chapter 8 Economic evaluation of endothelial keratoplasty techniques and penetrating keratoplasty in the Netherlands Am J Ophthalmol. 2012 Aug;154(2):272-281

Chapter 9 Femtosecond laser-assisted inverted mushroom keratoplasty 


\section{Part 2 Deep anterior lamellar keratoplasty}

Chapter 10 Endothelial cell loss and visual outcome of deep anterior lamellar keratoplasty versus penetrating keratoplasty: a randomized multicenter clinical trial

Ophthalmology. $2011 \mathrm{Feb}$;118(2):302-9

Chapter 11 Quality of vision after deep anterior lamellar keratoplasty versus penetrating keratoplasty: a randomized, multicenter clinical trial

Submitted

Chapter 12 Economic evaluation of deep anterior lamellar keratoplasty 207 versus penetrating keratoplasty in The Netherlands Am J Ophthalmol. 2011 Mar;151(3):449-59

Chapter 13 General discussion 229

Chapter 14 Valorization addendum 237

Summary/Samenvatting 245

Dankwoord

Curriculum vitae 263

List of publications 


\section{LIST OF ABBREVIATIONS}

\begin{tabular}{|c|c|}
\hline$A B K$ & aphakic bullous keratopathy \\
\hline ABKP & aphakic bullous keratopathy \\
\hline AC-IOL & anterior chamber intraocular lens \\
\hline AMD & age-related macular degeneration \\
\hline BCVA & best corrected visual acuity \\
\hline BIS & bio implant services \\
\hline BSCVA & best spectacle-corrected visual acuity \\
\hline CEACs & cost-effectiveness acceptability curves \\
\hline $\mathrm{Cl}$ & confidence interval \\
\hline CME & cystoid macular edema \\
\hline $\mathrm{D}$ & diopters \\
\hline DLCTS & Dutch lamellar corneal transplantation study \\
\hline DLEK & deep lamellar endothelial keratoplasty \\
\hline DLK & deep lamellar keratoplasty \\
\hline DMAEK & Descemet's membrane automated endothelial keratoplasty \\
\hline DSAEK & Descemet's stripping automated endothelial keratoplasty \\
\hline DSEK & Descemet's stripping endothelial keratoplasty \\
\hline EC & endothelial cell \\
\hline ECD & endothelial cell density \\
\hline EK & endothelial keratoplasty \\
\hline ERM & epiretinal membrane \\
\hline ETDRS & early treatment of diabetic retinopathy study \\
\hline FLEK & femtosecond laser-assisted endothelial keratoplasty \\
\hline FS & femtosecond laser \\
\hline FS-DSEK & femtosecond laser-assisted Descemet's stripping endothelial keratoplasty \\
\hline Fuchs & Fuchs endothelial dystrophy \\
\hline ICER & incremental cost-effectiveness ratio \\
\hline IOL & intraocular lens \\
\hline IOP & intraocular pressure \\
\hline LASIK & laser in situ keratoileusis \\
\hline $\log (c)$ & logarithm of contrast sensitivity \\
\hline $\log (s)$ & logarithmic intraocular straylight value \\
\hline LogMAR & logarithm of the minimum angle of resolution \\
\hline MUMC & Maastricht university medical center \\
\hline NA & not applicable \\
\hline NEI VFQ-25 & national eye institute visual functioning questionnaire- 25 \\
\hline NS & not significant \\
\hline
\end{tabular}


PBK

pseudophakic bullous keratopathy

PK penetrating keratoplasty

PKP penetrating keratoplasty

PLD posterior lamellar disc

PLK posterior lamellar keratoplasty

QALYs quality-adjusted life years

RCT randomized clinical trial

$\mathrm{RD}$ retinal detachment

RPE retinal pigment epithelium

SD standard deviation

SEM scanning electron microscopy

TEM transmission electron microscopy

UCVA uncorrected visual acuity

UT ultrathin 



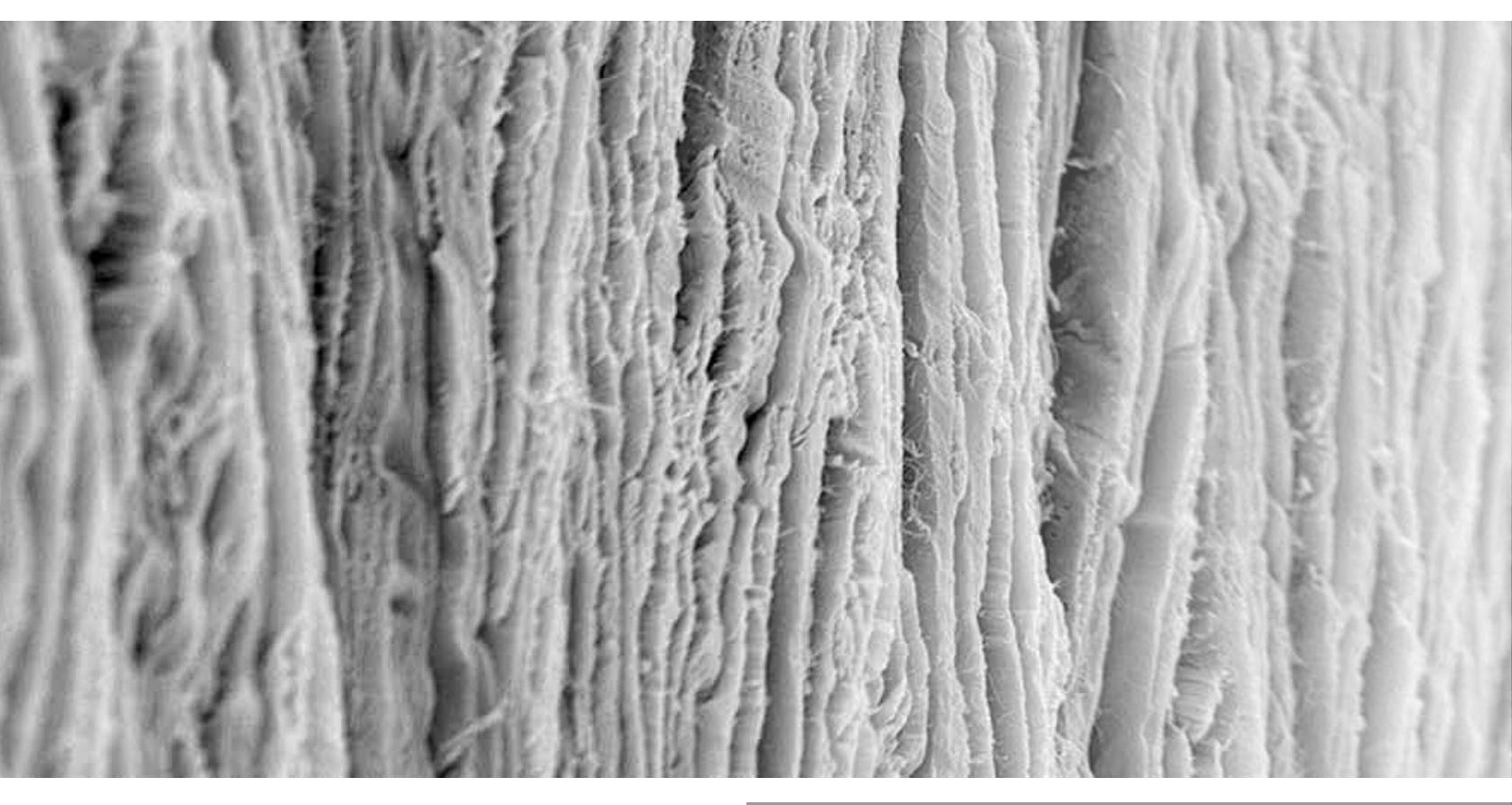


CHAPTER 1

General Introduction 



\section{ANATOMY OF THE HUMAN CORNEA}

The cornea is a transparent, avascular tissue and serves as a crystal clear "window" of the eye that allows the entry of light. The size of an adult human cornea is 11 to 12 $\mathrm{mm}$ horizontally and 9 to $10 \mathrm{~mm}$ vertically. Central corneal thickness is about $0.5 \mathrm{~mm}$, increasing slightly toward the periphery, where it is about $0.7 \mathrm{~mm}{ }^{1,2}$ The cornea consists of three different cellular layers and two interfaces in between, from anterior to posterior: epithelium, Bowman's layer, stroma, Descemet's membrane, and the endothelium (Figure 1). Recently, a novel acellular pre-Descemet's layer has been reported (Dua's layer). ${ }^{3}$

Optimal corneal optics requires a smooth surface with healthy tear film and epithelium. The corneal transparency is mainly dependent on the stroma and endothelium. The stroma forms the largest portion, about $90 \%$, of the thickness of the cornea. The stroma is covered at the anterior side by epithelium, and the interface between epithelium and stroma is the Bowman's layer. The Bowman's layer does not regenerate after damage, and the function of this layer remains uncertain. The stroma consists of an uniform arrangement of collagen fibers, which is essential for corneal transparency. ${ }^{2}$ The posterior side of the stroma is covered by Descemet's membrane and endothelium. Descemet's membrane becomes thicker throughout life; it is $3 \mu \mathrm{m}$ at birth and increases to $10 \mu \mathrm{m}$ in adulthood. ${ }^{4}$ Corneal endothelium is a single layer and covers the posterior surface

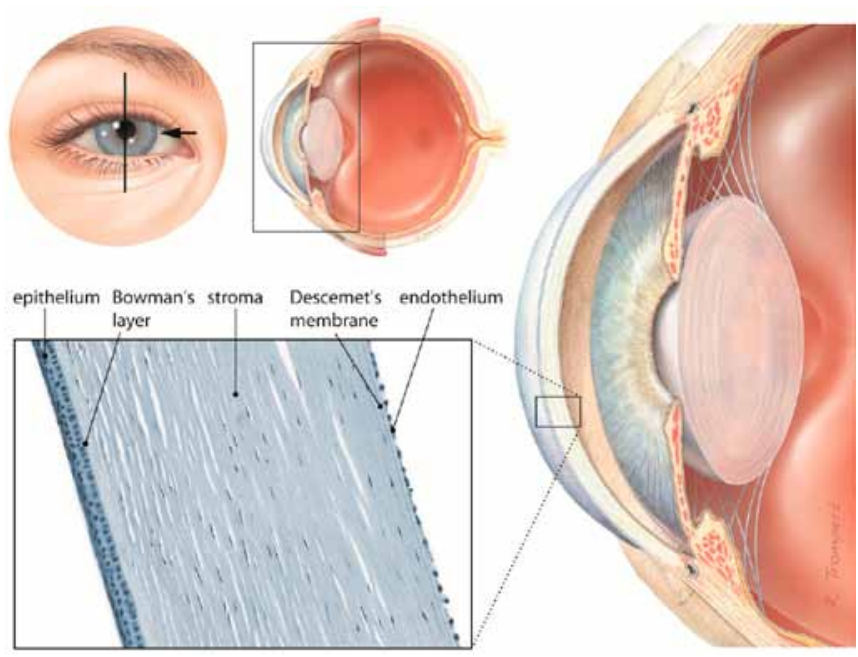

Figure 1. Anatomy of the Human Cornea 
of Descemet's membrane. Normal endothelium consists primarily of hexagonal cells, and after loss or damage to the endothelium, the hexogonality value decreases and variability of cell area increases. Endothelial cell density is about 3500 to 4000 cells $/ \mathrm{mm}^{2}$ at birth and decreases with a physiologic rate of $0.6 \%$ per year to 2500 to 3000 cells $/ \mathrm{mm}^{2}$ in older age. ${ }^{5,6}$ Further, an intact corneal endothelium is important for maintenance of stromal transparency, since the endothelium regulates corneal hydration. Endothelial function becomes impaired when cell numbers decline to $300-600$ cells $/ \mathrm{mm}^{2,7}$

\section{CORNEAL TRANSPLANTATION}

\subsection{History of corneal transplantation}

In 1824 it was Franz Riesinger who was the first to suggest replacing human opaque cornea with transparent animal cornea, and used the term "keratoplasty". ${ }^{8}$ Unfortunately, none of the corneas remained clear. After several decades of experiment and development, finally the first successful human penetrating corneal transplantation was performed by Dr. Eduard Zirm in 1906. ${ }^{9}$ He had suggested a few important points for a successful keratoplasty: use of young and healthy human corneas, use of the von Hippel trephine, anesthesia, protection of the graft, and use of overlay sutures. ${ }^{10}$

Since this first successful report of penetrating corneal transplantation (Figure 2A), various clinical and laboratory research had been performed to develop new trephine models, suturing techniques, and surgical instruments." The introduction of topical

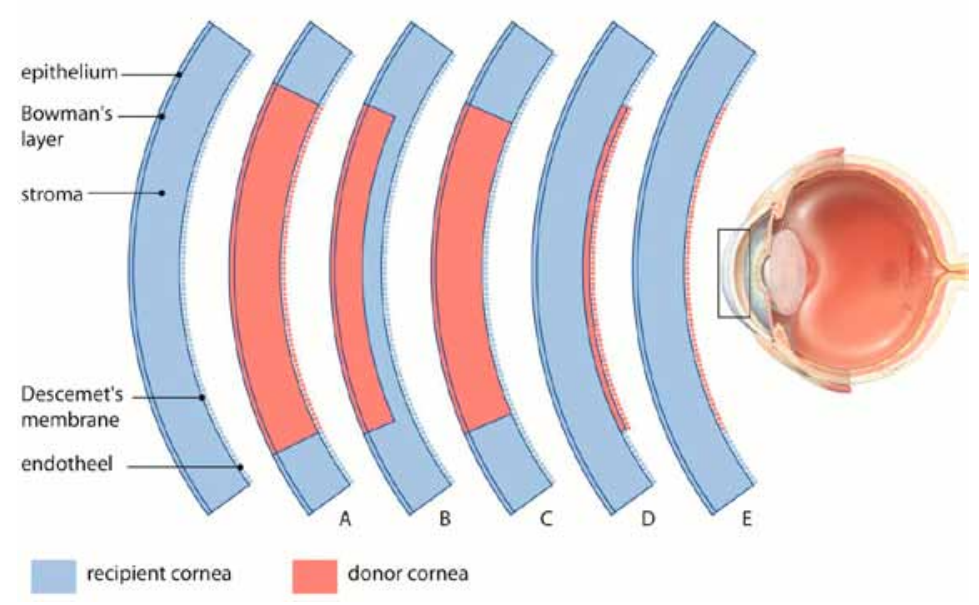

Figure 2. Different Types of Cornea Transplantation

A = Penetrating Keratoplasty, B = Anterior Lamellar Keratoplasty, C = Deep Anterior Lamellar Keratoplasty , D = Descemet's Stripping Automated Endothelial Keratoplasty, E = Descemet's Membrane Endothelial Keratoplasty 
antibiotics and corticosteroids had a profound impact on the success rate of corneal graft survival. Worldwide, human corneas are the most commonly transplanted tissue, and corneal transplantations are the most successful type of tissue transplantation.

During the last decade, different types of lamellar corneal transplantation surgery have been described (such as anterior lamellar keratoplasty and endothelial keratoplasty), which selectively replace only the diseased layers of the cornea (Figure 2). These lamellar techniques have replaced a great part of the standard penetrating techniques. In 2012, 46102 corneal transplantations were performed in the United States. ${ }^{12}$ The number of penetrating keratoplasty (PK) had decreased from 42063 in 2005 to 21422 in 2012, and the number of endothelial keratoplasties increased from 1398 in 2005 to 23049 in 2012, which was the most performed type of lamellar keratoplasty in 2012. Anterior lamellar keratoplasty showed a slight increase of 641 in 2005 to 883 in 2012, but it remains lower in comparison with PK. In the Netherlands, the reported number of corneal transplantations in 2012 increased from 1290 to1401 (9\%). ${ }^{13}$

\section{PENETRATING KERATOPLASTY}

\subsection{Indications for penetrating keratoplasty}

Until the last century, full thickness PK was the gold standard treatment for various corneal diseases. In PK, the recipient full-thickness corneal tissue is replaced with fullthickness donor corneal tissue. The goals of PK were visual rehabilitation, tectonic support, reduction of pain, prevention of infection and cosmetic restoration. The leading indications were keratoconus, pseudophakic bullous keratopathy, Fuchs' endothelial dystrophy and regrafts. Other less common indications were post-infectious scarring, diverse corneal dystrophies, perforating corneal injury or chemical burns. ${ }^{12,}{ }^{14-20}$ PK has been shown to be a successful treatment for restoration of vision in eyes with corneal disease. In general, PK results in a clear corneal graft with a graft survival rate of $90 \%$ at 1 year follow-up and up to $72 \%$ after 5 years follow-up. ${ }^{14,21}$ Few PK studies have reported up to 10-years follow-up, and the 10-year graft survival rate has been reported to be $50 \%$ to over $90 \%$. $^{22-27}$

\subsection{Clinical outcomes and complications of penetrating keratoplasty}

Over the last century, several technological improvements have substantially reduced the incidence of early postoperative complications, but adverse effects including high irregular astigmatism, suture-related events, wound healing problems, allograft rejection, graft failure and raised intraocular pressure still remain challenging. ${ }^{14,21,28}$ 


\section{Astigmatism}

By the late 1800 's, von Hippel designed a circular mechanical trephine to remove the donor and recipient corneas. His principle is still widely used today. In PK, the recipient cornea is trephined in the vertical direction and the donor cornea is sutured into the recipient bed. Depending on the strength, length or depth, sutures may result in high and/or irregular postoperative astigmatism, and limit postoperative useful vision for patients. A variety of suturing techniques, such as interrupted sutures, a running suture, a combined interrupted and running sutures, and double running sutures have been described. However, no single suturing technique has proven superior in order to control final suture-out astigmatism. ${ }^{29-31}$

Other factors that may also influence postoperative PK astigmatism include graft size, $^{32,}{ }^{33}$ donor-recipient disparity, ${ }^{29}$ oval or eccentric trephination, ${ }^{34,}{ }^{35}$ and timing of suture removal or adjustment. ${ }^{36}$ Larger corneal grafts tend to be related with lower astigmatism and higher rejection rates. In contrast, a smaller corneal graft results in a lower rejection rate and a higher degree of topographic irregularity. ${ }^{33}$

Post-PK astigmatism greater than 3.0 diopters has been reported in more than $40 \%$ of eyes, leading to anisometropia and image distortion, and are not correctable with spectacles. ${ }^{21,37,38}$ Both postoperative selective suture removal and suture adjustment can be used to reduce astigmatism, but astigmatism after suture removal is very variable. Other treatment options for postoperative irregular astigmatism and ametropia are rigid gas permeable contact lenses, arcuate corneal relaxing incisions, ${ }^{39}$ corneal laser refractive surgery, ${ }^{40}$ and implantation of toric intraocular lenses. ${ }^{41}$

\section{Suture-related problems}

Sutures are needed to keep the donor cornea in place, and different suture-related complications have been reported. Breaking or loosening of a suture may result in pain, excess mucus production, filamentary keratitis, corneal ulceration, sterile infiltrates, and wound dehiscence. ${ }^{42}$ Loose sutures increase the risk of inflammation and allograft rejection, ${ }^{43}$ and therefore, broken or loose sutures should be removed immediately.

\section{Wound dehiscence}

Wound dehiscence may appear after suture removal or traumatic accident. ${ }^{44}$ Different graft configurations have been described to achieve a stronger corneal wound. In 1951, Francheschetti described the first mushroom configuration graft, ${ }^{45}$ and later Busin popularized the inverted mushroom configuration (later coined top-hat configuration) by using a mechanical trephination technique. ${ }^{46}$ The idea was to combine the advantages of a clear optical center of the donor graft and the increased wound healing of a lamellar graft in the periphery. However, the manual trephination technique has not gained widespread use, mainly because of the surgical difficulties involved with the 
manual dissection. Seitz et al. were the first to report the feasibility of an industrial femtosecond laser to create an inverse mushroom shape in polymethylmethacrylate blocks and porcine corneas. ${ }^{47}$ After the introduction of femtosecond laser it became possible to create different trephination profiles. Using femtosecond laser technology, precise corneal incisions with customized graft edges and lamellar planes for both donor and recipient corneas, such as top-hat, mushroom, zigzag and Christmas tree configurations can be constructed. It has been suggested that these trephination profiles offer a better donor-recipient fit and increase donor-host junction surface contact, resulting in faster wound healing with a stronger wound, earlier suture removal, and lower postoperative astigmatism. ${ }^{48-52}$ However, there are no randomized clinical trial studies demonstrating a superior clinical outcome in comparison to PK.

\section{Allograft rejection}

Corneal transplantation is the oldest and most successful human tissue transplantation with a high percentage of graft survival (up to $90 \%$ at 1 year). ${ }^{14,21}$ It has been observed that donor corneas were rejected less frequently than other transplanted tissue. This led to the concept that the cornea is an immune privileged tissue with a reduced incidence in immune corneal allograft rejection. ${ }^{53}$ However, corneal allograft rejection is one of the most important causes of corneas graft failure. ${ }^{23,54,55}$

The three layers of the cornea (epithelium, stroma, and endothelium) may show allograft rejection either individually or simultaneously. Epithelial rejection has been reported at a rate of $10 \%$ of patients with rejection and it is usually seen in the early postoperative period (1-13 months). ${ }^{56}$ It appears as an elevated, linear epithelial ridge from the periphery towards the center of the graft. Distinct subepithelial infiltrates in the central of the cornea may manifest as corneal graft rejection. Stromal rejection is uncommon and it may visible as stromal infiltrates and neovascularisation. The characteristic signs of endothelial rejection are a linear line from the peripheral cornea towards the center (Khodadoust line) or randomly fine endothelial keratic precipitates on the endothelial surface. ${ }^{57}$ The most important and serious allograft rejection is endothelial rejection, because the endothelial cells can be damaged and are not able to restore. It is very important to recognize the early signs of allograft rejection and to start aggressive therapy, because most of the allograft rejection can be reserved.

\section{Graft failure}

The important factors of corneal graft failure are endothelial rejection and late endothelial failure, which represent more than $50 \%$ of graft failures. ${ }^{23,55}$ The late endothelial failure was defined as a gradual decrease in graft transparency without evident cause, unresponsive to corticosteroid therapy, and not related to a recent episode of graft rejection. Endothelial cell density is known to decrease over time with a physiologic 
rate of $0.6 \%$ per year after age $18,{ }^{5}$ and endothelial cell loss after PK is faster than the physiologic rate. ${ }^{23}$ The reported endothelial cell loss with a follow-up of 1 year, 5 years, 10 years and 15 years are 34\%,59\%,67\% and $71 \%$, respectively. ${ }^{55}$ Furthermore, another important risk factor for graft failure is glaucoma, since elevated intraocular pressure may result in endothelial cell damage. ${ }^{58,59}$

\section{ANTERIOR LAMELLAR KERATOPLASTY}

\subsection{History and indications of anterior lamellar keratoplasty}

The concept for anterior lamellar keratoplasty was first described in 1840 by Franz Mühlbauer. ${ }^{60}$ Whereas in PK all five layers of the cornea are transplanted, in anterior lamellar keratoplasty the recipient corneal stroma is partially or totally removed to preserve Descemet's membrane and the endothelium, and the corneal stroma is replaced with a lamellar donor cornea button. In the 1970s, there was an increased interest in anterior lamellar keratoplasty, but due to the technical difficulty of the procedure and the reduced postoperative acuity, PK remained the standard procedure. ${ }^{61}$ Over the past 10 years, several innovative surgical techniques have been described which allow lamellar dissection of recipient and donor cornea. This procedure has been described as deep lamellar keratoplasty (DLK) and deep anterior lamellar keratoplasty (DALK), but the term DLK has widely used as "diffuse lamellar keratitis" after laser in situ keratomileusis surgery, so DLK is no longer used in anterior lamellar keratoplasty. ${ }^{62,} 63$

The surgical challenge of anterior lamellar keratoplasty is separating the anterior stromal layers from Descemet's membrane and endothelium. Various techniques have been described to achieve baring of Descemet's membrane, including intrastromal air injection, ${ }^{64}$ hydrodelamination, ${ }^{63}$ viscoelastic dissection using an air-to-endothelium interface, ${ }^{65}$ blunt spatula delamination, ${ }^{66}$ femtosecond laser-assisted, ${ }^{67,68}$ and the most widely used technique is the "big-bubble" of Anwar and Teichmann. ${ }^{66}$ Recently, anterior segment optical coherence tomography-guided big-bubble technique has also been described. ${ }^{69,70}$

Indications for DALK are corneal pathologies without involvement of the endothelium, such as keratoconus, corneal scarring related to infectious keratitis, and stromal corneal dystrophies. $^{71}$

\subsection{Advantages of anterior lamellar keratoplasty}

DALK has several advantages over PK. The main advantage of DALK is preservation of the recipient's own endothelium, while replacing epithelium and corneal stroma. This eliminates the risk of endothelial allograft rejection, which is the major cause of graft failure in PK surgery. ${ }^{55}$ Furthermore, long-term endothelial cell loss is lower and the pre- 
dicted graft survival is higher after DALK than after PK. ${ }^{72}$ Other proposed advantages of DALK include shorter postoperative steroid therapy, reduced risk of intraocular infection due to a "closed eye" procedure, better wound stability, and earlier suture removal. ${ }^{73-75}$

\subsection{Clinical outcomes of anterior lamellar keratoplasty}

The two main outcome parameters of corneal grafting are visual outcome and graft survival. A significantly better best-corrected visual acuity (BCVA) after DALK has been reported, ${ }^{76}$ but the majority of comparative studies show no significant difference in the postoperative best spectacle-corrected visual acuity (BSCVA) between DALK and PK. ${ }^{62}$, 73, 77-83 Only some studies show a significant better BSCVA after PK compared to DALK. ${ }^{74,}$ ${ }^{84}$ Visual outcomes of DALK may be limited compared with PK if baring of Descemet's membrane is incomplete or interface haze occurs. It has been suggested that the main parameter for good visual outcome after DALK is the thickness of residual recipient stromal bed. ${ }^{84,85}$ An eye with DALK with a residual stromal bed of less than $20 \mu \mathrm{m}$ has been shown to give similar visual results as an eye after PK. Deep dissection up to the level of Descemet's membrane is thought to lead to better visual outcomes because of the absence of stromal scarring at the level of the graft-host stromal interface. ${ }^{63}$

There is no significant difference in postoperative astigmatism and spherical equivalent for DALK versus PK in several published studies. ${ }^{75}{ }^{83}$ Other outcomes than standard visual outcomes, such as glare testing, contrast sensitivity, intraocular straylight, and wavefront analysis have been reported by several studies. ${ }^{62,73,80,84,86-89}$

Graft survival is generally determined in the majority of cases by endothelial rejection or late failure. As mentioned above, the advantage of DALK is preservation of the own endothelium, which eliminates the risk of endothelial allograft rejection. Randomized and non-randomized clinical trials have compared postoperative endothelial cell loss between DALK and PK, and showed a significantly higher endothelial cell loss after PK. ${ }^{72}$ 75,83 Studies have also shown a trend in which most of endothelial cell loss occurs in the first 6 months after a DALK procedure. ${ }^{75}$ Borderie et al were the first who used a joint regression model to predict long-term graft survival of DALK versus PK, and showed a higher median predicted graft survival in the DALK group. ${ }^{72}$ This means that a young patient undergoing DALK should not require regrafting in the long term. On the other hand, regrafting is likely to be necessary in the long term for a young patient undergoing PK.

Immune-mediated endothelium rejection cannot occur after DALK, but epithelial rejection and stromal rejection may still occur following DALK. ${ }^{90}$ This can result in significant corneal scarring with decreased graft clarity and decreased visual outcome. 


\subsection{Complications unique to anterior lamellar keratoplasty}

The main complication of DALK procedure is intraoperative perforation of the Descemet's membrane, which occurs in approximately $4 \%$ to $39 \% .{ }^{75}$ Large perforations (macroperforations) require convertion to a PK procedure intraoperatively (2.0 to $18 \%){ }^{75,83}$ Small perforations (microperforations) can usually be managed with air or gas injection into the anterior chamber. The occurrence of a double anterior chamber postoperatively is generally related to a perforation of Descemet's membrane. Although spontaneous attachment of Descemet's membrane might occur, usually air injection in the anterior chamber is required. Other uncommon interface related complications after DALK are debris, hemorrhage, vascularization, infection, and epithelial ingrowth. ${ }^{75}$

\section{ENDOTHELIAL KERATOPLASTY}

\subsection{History and indications of endothelial keratoplasty}

The concept of selective tissue replacement for the posterior cornea has been described for many decades. The first successful endothelial keratoplasty was reported by Charles Tillet in 1956. ${ }^{91}$ Although Tillet's endothelial keratoplasty was successful, no further cases were reported for decades. In 1998, Melles et al described a revised technique of inserting a donor tissue through a limbal incision, and injection of an air bubble in the anterior chamber to initiate self-adherence of the donor tissue without the need for sutures. ${ }^{92}$ This technique was first described by Melles as posterior lamellar keratoplasty (PLK). In 2000, Terry et al modified this technique, and called it deep lamellar endothelial keratoplasty (DLEK). ${ }^{93}$ Despite the improvement in the two techniques, PLK and DLEK were not widely practiced due to the technical challenges. In both techniques, it requires manual lamellar dissection for both the posterior recipient cornea and the preparation of the donor lenticule. Continued development of newer techniques, such as stripping of the Descemet's membrane from the recipient, "Descemetorrhexis", resulted in a smoother recipient interface. ${ }^{94}$ This procedure has been popularized as Descemets's stripping endothelial keratoplasty (DSEK). ${ }^{95}$ The use of an automated microkeratome has replaced the difficult hand-dissection donor cornea preparation and this provides a smooth deep lamellar cut in the donor cornea. ${ }^{96}$ This procedure was described as Descemet's stripping automated endothelial keratoplasty (DSAEK) by Gorovoy. ${ }^{96}$ Afterwards, preparation of the donor cornea for endothelial keratoplasty with femtosecond laser has also been described. ${ }^{97}$ Recently, transplantation of only Descemet's membrane with endothelium has been named Descemet's membrane endothelial keratoplasty (DMEK) by Melles, ${ }^{98}$ and Descemet's membrane automated endothelial keratoplasty (DMAEK) by McCauley et al. ${ }^{99}$ 
Indications for endothelial keratoplasty are corneas with endothelial cell dysfunction and a healthy anterior recipient cornea. Currently in the United States, endothelial dystrophy (e.g. Fuchs endothelial dystrophy) and pseudophakic bullous keratopathy are the two most important indications for endothelial keratoplasty. ${ }^{12}$ Other less common indications are posterior polymorphous dystrophy, iridocorneal endothelial syndrome, endothelial decompensation due to trauma, glaucoma devices, chronic iritis, or failed PK. ${ }^{100,101}$

According to the Eye bank of America Association 2012 statistical report, DSEK/DSAEK ( 23049 cases) is at present the most performed procedure of endothelial keratoplasty in the United States, and DMEK has been increased from 344 cases in 2011 to 748 cases in 2012. ${ }^{12}$

\subsection{Advantages of endothelial keratoplasty}

The major advantage of all currently used endothelial keratoplasty procedures are that no sutures are required to keep the donor tissue in place, thereby preventing the occurrence of suture- or incision-induced high and irregular astigmatism, resulting in faster visual rehabilitation, more predictable postoperative corneal power, better wound stability, minimal suture-related complications. ${ }^{102}$ It is a closed eye surgery which also reduces the risk of preoperative expulsive hemorrhage. Further, the reported Kaplan-Meier cumulative probability of immunologic rejection at 1 year follow-up was $1 \%, 8 \%$ and $14 \%$ for DMEK, DSEK and PK, respectively. ${ }^{103}$ The immunologic rejection rate is significantly reduced in thinner graft, but the rejection rate between Ultrathin-DSAEK and DMEK at 1 year is comparable. ${ }^{104}$

\subsection{Clinical outcomes of endothelial keratoplasty}

Endothelial keratoplasty has reported faster visual rehabilitation in comparison with PK. Despite the advantages of endothelial keratoplasty, visual acuity of the first large report of DLEK is limited to $20 / 46$ due to the graft-host interface. ${ }^{105}$ After introduction of Descemetorhexis and microkeratome, the interface smoothness improved significantly, and this resulted in a substantial improvement in quality of vision and rapidity of visual recovery. Numerous studies have reported better visual recovery after DMEK in comparison with DSAEK. Six months after DSAEK, $69-92 \%$ of eyes have a BCVA of $\geq 20 / 40$ and 11 $20 \%$ can obtain $\geq 20 / 20{ }^{106-109}$ In studies after DMEK, the percentage of eyes with BCVA $\geq 20 / 40$ and $\geq 20 / 20$ are $88-95 \%$ and $36-50 \%$ at 6 months follow-up, respectively. ${ }^{110-112}$

Another advantage of endothelial keratoplasty over PK is the amount of postoperative astigmatism. PK frequently results in unpredictable topography and high irregular astigmatism; this has changed considerably with endothelial keratoplasty. The average surgically induced astigmatism in a several large series of DSAEK was less than 0.25 diopters. ${ }^{106,107,}{ }^{109}$ Further, due to the minimal change in corneal topography, the anterior cor- 
nea curvature remained moderately unchanged, thereby causing only minimal change in spherical equivalent. Several endothelial keratoplasty procedures have reported a mild hyperopic shift. ${ }^{102,}{ }^{105,}{ }^{109}$ This results from the change in posterior curvature, since the donor lenticule is thinner in the center and thicker at the edges. Hyperopic shift has also been reported after DMEK, but this may result from corneal deturgescence instead of posterior corneal curvature change. ${ }^{113,114}$ Therefore, when performing cataract surgery in combination with endothelial keratoplasty or cataract surgery prior to endothelial keratoplasty, the power of the intraocular lens must be targeted at mild myopia of -1.0 to -1.25 diopters.

The main concern of both endothelial keratoplasty and PK is to maintain a high endothelial cell density, as it is a crucial factor for long-term graft survival. Mean endothelial cell loss after DSAEK ranges widely, from $13 \%$ to $50 \%$ at 6 months, $42 \%$ to $44 \%$ at 3 years, and $54 \%$ at 5 years. ${ }^{73,115-120121,122}$ After DMEK, the endothelial cell loss has been reported between $15 \%$ and $41 \%$ at 6 months, and between $39 \%$ and $55 \%$ at 5 years. ${ }^{111-113,}{ }^{123-125}$

\subsection{Complications unique to endothelial keratoplasty}

The most frequent complication of endothelial keratoplasty is dislocation of donor tissue. Dislocation rates are between $0 \%$ and $82 \%$, with an average of $15 \% .{ }^{126}$ Various technical modifications have evolved in order to reduce the incidence of donor dislocation, such as surface sweeping to remove interface fluid, longer postoperative supine positioning, 3 to 4 corneal stab incisions into the interface to release small amounts of interface fluid, and gentle scraping of the stripped surface of the peripheral recipient bed to expose the peripheral stromal fibrils and promote donor edge adhesion. ${ }^{127,}{ }^{128}$ Primary graft failure after DSEK has been reported to be higher than after PK, although this higher rate may be associated with the surgical learning curve in endothelial keratoplasty. ${ }^{126,129}$ Pupillary block is a rare complication that can develop after endothelial keratoplasty when an excessively large air bubble has been left in the anterior chamber. ${ }^{96,130}$ Finally, interface deposits and epithelial ingrowth have also been reported. ${ }^{131,132}$

\section{AIM AND OUTLINE OF THE THESIS}

The aim of this thesis is to compare the outcome of classical PK to anterior and posterior lamellar keratoplasty in randomized clinical studies and to evaluate the role of the femtosecond laser in performing lamellar transplantation surgery.

Chapter $\mathbf{2}$ and $\mathbf{3}$ evaluate the histologic changes in corneal structure and corneal endothelial viability after femtosecond laser preparation of posterior lamellar discs.

Chapter 4 describes the first case of femtosecond laser-assisted Descemet's stripping endothelial keratoplasty. 
Chapter $\mathbf{5}$ presents the preliminary visual results of patients after femtosecond laserassisted Descemet's stripping endothelial keratoplasty.

Chapter 6 discusses the visual outcomes, endothelial cell loss and complications of femtosecond laser-assisted Descemet's stripping endothelial keratoplasty versus penetrating keratoplasty in a randomized multicenter clinical trial.

Chapter $\mathbf{7}$ compares the straylight and contrast sensitivity after femtosecond laserassisted Descemet's stripping endothelial keratoplasty versus penetrating keratoplasty in a randomized multicenter clinical trial.

Chapter 8 evaluates the cost-effectiveness of penetrating keratoplasty, femtosecond laser-assisted Descemet stripping endothelial keratoplasty, and Descemet stripping automated endothelial keratoplasty.

Chapter 9 evaluates the 1-year visual outcomes and endothelial cell density of a case series of patients with femtosecond laser-assisted inverted mushroom keratoplasty.

Chapter 10 and 11 both discuss the results of endothelial cell loss, visual outcomes and quality of vision after deep anterior lamellar keratoplasty versus penetrating keratoplasty in a randomized multicenter clinical trial.

Chapter 12 evaluates the cost-effectiveness of deep anterior lamellar keratoplasty versus penetrating keratoplasty in a randomized multicenter clinical trial. 


\section{REFERENCES}

1. Mishima S. Corneal thickness. Surv Ophthalmol 1968;13(2):57-96.

2. Freegard TJ. The physical basis of transparency of the normal cornea. Eye (Lond) 1997;11 ( Pt 4):465-471.

3. Dua HS, Faraj LA, Said DG, Gray T, Lowe J. Human corneal anatomy redefined: a novel pre-Descemet's layer (Dua's layer). Ophthalmology 2013;120(9):1778-1785.

4. Johnson DH, Bourne WM, Campbell RJ. The ultrastructure of Descemet's membrane. I. Changes with age in normal corneas. Arch Ophthalmol 1982;100(12):1942-1947.

5. Bourne WM, Nelson LR, Hodge DO. Central corneal endothelial cell changes over a ten-year period. Invest Ophthalmol Vis Sci 1997;38(3):779-782.

6. Yee RW, Matsuda M, Schultz RO, Edelhauser HF. Changes in the normal corneal endothelial cellular pattern as a function of age. Current eye research 1985;4(6):671-678.

7. Hoffer KJ. Corneal decomposition after corneal endothelium cell count. Am J Ophthalmol 1979;87(2):252-253.

8. Reisinger F. Die Keratoplastik: ein Versuch zur Erweiterung der Augenheilkunst. Bayerische Annalen 1824;1:207-215.

9. Zirm EK. Eine erfolgreiche totale Keratoplastik (A successful total keratoplasty). 1906. Refract Corneal Surg 1989;5(4):258-261.

10. Moffatt SL, Cartwright VA, StumpfTH. Centennial review of corneal transplantation. Clin Experiment Ophthalmol 2005;33(6):642-657.

11. Castroviejo R. Instrumentation and techniques of keratoplasty. Trans Am Acad Ophthalmol Otolaryngol 1960;64:775-785.

12. 2012 Eye Banking Statistical Report Eye Bank Association of America. www.restoresight.org

13. Nederlandse Transplantatie Stichting. Jaarverslag 2011. www.transplantatiestichting.nl/cijfersweefsels.

14. Williams KA, Esterman AJ, Bartlett C, Holland H, Hornsby NB, Coster DJ. How effective is penetrating corneal transplantation? Factors influencing long-term outcome in multivariate analysis. Transplantation 2006;81(6):896-901.

15. Beckingsale P, Mavrikakis I, Al-Yousuf N, Mavrikakis E, Daya SM. Penetrating keratoplasty: outcomes from a corneal unit compared to national data. Br J Ophthalmol 2006;90(6):728-731.

16. Claesson M, Armitage WJ, Fagerholm P, Stenevi U. Visual outcome in corneal grafts: a preliminary analysis of the Swedish Corneal Transplant Register. Br J Ophthalmol 2002;86(2):174-180.

17. Mannis MJ, Holland EJ, Beck RW, et al. Clinical profile and early surgical complications in the Cornea Donor Study. Cornea 2006;25(2):164-170.

18. Sony P, Sharma N, Sen S, Vajpayee RB. Indications of penetrating keratoplasty in northern India. Cornea 2005;24(8):989-991.

19. Fasolo A, Frigo AC, Bohm E, et al. The CORTES study: corneal transplant indications and graft survival in an Italian cohort of patients. Cornea 2006;25(5):507-515.

20. Tan DT, Janardhanan P, Zhou H, et al. Penetrating keratoplasty in Asian eyes: the Singapore Corneal Transplant Study. Ophthalmology 2008;115(6):975-982 e971.

21. Williams KA, Muehlberg SM, Lewis RF, Coster DJ. How successful is corneal transplantation? A report from the Australian Corneal Graft Register. Eye (Lond) 1995;9 ( Pt 2):219-227.

22. Thompson RW, Jr., Price MO, Bowers PJ, Price FW, Jr. Long-term graft survival after penetrating keratoplasty. Ophthalmology 2003;110(7):1396-1402. 
23. Ing JJ, Ing HH, Nelson LR, Hodge DO, Bourne WM. Ten-year postoperative results of penetrating keratoplasty. Ophthalmology 1998;105(10):1855-1865.

24. Muraine M, Sanchez C, Watt L, Retout A, Brasseur G. Long-term results of penetrating keratoplasty. A 10-year-plus retrospective study. Graefes Arch Clin Exp Ophthalmol 2003;241(7):571-576.

25. Fukuoka S, Honda N, Ono K, Mimura T, Usui T, Amano S. Extended long-term results of penetrating keratoplasty for keratoconus. Cornea 2010;29(5):528-530.

26. Kelly TL, Williams KA, Coster DJ, Australian Corneal Graft R. Corneal transplantation for keratoconus: a registry study. Arch Ophthalmol 2011;129(6):691-697.

27. Niziol LM, Musch DC, Gillespie BW, Marcotte LM, Sugar A. Long-term outcomes in patients who received a corneal graft for keratoconus between 1980 and 1986. Am J Ophthalmol 2013;155(2):213219 e213.

28. Nagra PK, Hammersmith KM, Rapuano CJ, Laibson PR, Cohen EJ. Wound dehiscence after penetrating keratoplasty. Cornea 2006;25(2):132-135.

29. Karabatsas CH, Cook SD, Figueiredo FC, Diamond JP, Easty DL. Combined interrupted and continuous versus single continuous adjustable suturing in penetrating keratoplasty: a prospective, randomized study of induced astigmatism during the first postoperative year. Ophthalmology 1998;105(11):1991-1998.

30. Spadea L, Cifariello F, Bianco G, Balestrazzi E. Long-term results of penetrating keratoplasty using a single or double running suture technique. Graefes Arch Clin Exp Ophthalmol 2002;240(5):415419.

31. Javadi MA, Naderi M, Zare M, Jenaban A, Rabei HM, Anissian A. Comparison of the effect of three suturing techniques on postkeratoplasty astigmatism in keratoconus. Cornea 2006;25(9):10291033.

32. Vail A, Gore SM, Bradley BA, Easty DL, Rogers CA, Armitate WJ. Clinical and surgical factors influencing corneal graft survival, visual acuity, and astigmatism. Corneal Transplant Follow-up Study Collaborators. Ophthalmology 1996;103(1):41-49.

33. Seitz B, Langenbucher A, Kuchle M, Naumann GO. Impact of graft diameter on corneal power and the regularity of postkeratoplasty astigmatism before and after suture removal. Ophthalmology 2003;110(11):2162-2167.

34. Riddle HK, Jr., Parker DA, Price FW, Jr. Management of postkeratoplasty astigmatism. Curr Opin Ophthalmol 1998;9(4):15-28.

35. Cohen KL, Holman RE, Tripoli NK, Kupper LL. Effect of trephine tilt on corneal button dimensions. Am J Ophthalmol 1986;101(6):722-725.

36. Burk LL, Waring GO, 3rd, Radjee B, Stulting RD. The effect of selective suture removal on astigmatism following penetrating keratoplasty. Ophthalmic Surg 1988;19(12):849-854.

37. Frost NA, Wu J, Lai TF, Coster DJ. A review of randomized controlled trials of penetrating keratoplasty techniques. Ophthalmology 2006;113(6):942-949.

38. Brooks SE, Johnson D, Fischer N. Anisometropia and binocularity. Ophthalmology 1996;103(7):11391143.

39. Geggel HS. Arcuate relaxing incisions guided by corneal topography for postkeratoplasty astigmatism: vector and topographic analysis. Cornea 2006;25(5):545-557.

40. Vajpayee RB, Sharma N, Sinha R, Bhartiya P, Titiyal JS, Tandon R. Laser in-situ keratomileusis after penetrating keratoplasty. Surv Ophthalmol 2003;48(5):503-514.

41. Nuijts RM, Abhilakh Missier KA, Nabar VA, Japing WJ. Artisan toric lens implantation for correction of postkeratoplasty astigmatism. Ophthalmology 2004;111(6):1086-1094. 
42. Christo CG, van Rooij J, Geerards AJ, Remeijer L, Beekhuis WH. Suture-related complications following keratoplasty: a 5-year retrospective study. Cornea 2001;20(8):816-819.

43. Abou-Jaoude ES, Brooks M, Katz DG, Van Meter WS. Spontaneous wound dehiscence after removal of single continuous penetrating keratoplasty suture. Ophthalmology 2002;109(7):1291-1296; discussion 1297.

44. Williams MA, Gawley SD, Jackson AJ, Frazer DG. Traumatic graft dehiscence after penetrating keratoplasty. Ophthalmology 2008;115(2):276-278 e271.

45. Franceschetti A. [Combined lamellar and perforant keratoplasty (mushroom graft)]. Bull Schweiz Akad Med Wiss 1951;7(2):134-145.

46. Busin M. A new lamellar wound configuration for penetrating keratoplasty surgery. Arch Ophthalmol 2003;121(2):260-265

47. Seitz B, Brunner H, Viestenz A, et al. Inverse mushroom-shaped nonmechanical penetrating keratoplasty using a femtosecond laser. Am J Ophthalmol 2005;139(5):941-944.

48. Buratto $L$, Bohm E. The use of the femtosecond laser in penetrating keratoplasty. Am J Ophthalmol 2007;143(5):737-742.

49. Farid M, Kim M, Steinert RF. Results of penetrating keratoplasty performed with a femtosecond laser zigzag incision initial report. Ophthalmology 2007;114(12):2208-2212.

50. Price FW, Jr., Price MO. Femtosecond laser shaped penetrating keratoplasty: one-year results utilizing a top-hat configuration. Am J Ophthalmol 2008;145(2):210-214.

51. Bahar I, Kaiserman I, Lange AP, et al. Femtosecond laser versus manual dissection for top hat penetrating keratoplasty. Br J Ophthalmol 2009;93(1):73-78.

52. Bahar I, Kaiserman I, McAllum P, Rootman D. Femtosecond laser-assisted penetrating keratoplasty: stability evaluation of different wound configurations. Cornea 2008;27(2):209-211.

53. Niederkorn JY. Corneal transplantation and immune privilege. International reviews of immunology 2013;32(1):57-67.

54. Khodadoust AA. The allograft rejection reaction: the leading cause of late failure of clinical corneal grafts. In: Corneal graft failure CIBA symposium Elsevier: Amsterdam 1973:151-167.

55. Patel SV, Hodge DO, Bourne WM. Corneal endothelium and postoperative outcomes 15 years after penetrating keratoplasty. Am J Ophthalmol 2005;139(2):311-319.

56. Alldredge $\mathrm{OC}$, Krachmer JH. Clinical types of corneal transplant rejection. Their manifestations, frequency, preoperative correlates, and treatment. Arch Ophthalmol 1981;99(4):599-604.

57. Khodadoust AA, Silverstein AM. Transplantation and rejection of individual cell layers of the cornea. Invest Ophthalmol 1969;8(2):180-195.

58. Reinhard T, Kallmann C, Cepin A, Godehardt E, Sundmacher R. The influence of glaucoma history on graft survival after penetrating keratoplasty. Graefes Arch Clin Exp Ophthalmol 1997;235(9):553557.

59. Price MO, Thompson RW, Jr., Price FW, Jr. Risk factors for various causes of failure in initial corneal grafts. Arch Ophthalmol 2003;121(8):1087-1092.

60. Geerling G, Duncker GI, Krumeich J, Melles GR. [Lamellar keratoplasty. Back to the future?!]. Der Ophthalmologe : Zeitschrift der Deutschen Ophthalmologischen Gesellschaft 2005;102(12):11401148, 1150-1141.

61. Richard JM, Paton D, Gasset AR. A comparison of penetrating keratoplasty and lamellar keratoplasty in the surgical management of keratoconus. Am J Ophthalmol 1978;86(6):807-811.

62. Shimazaki J, Shimmura S, Ishioka M, Tsubota K. Randomized clinical trial of deep lamellar keratoplasty vs penetrating keratoplasty. Am J Ophthalmol 2002;134(2):159-165. 
63. Sugita J, Kondo J. Deep lamellar keratoplasty with complete removal of pathological stroma for vision improvement. Br J Ophthalmol 1997;81(3):184-188.

64. Archila EA. Deep lamellar keratoplasty dissection of host tissue with intrastromal air injection. Cornea 1984;3(3):217-218.

65. Melles GR, Lander F, Rietveld FJ, Remeijer L, Beekhuis WH, Binder PS. A new surgical technique for deep stromal, anterior lamellar keratoplasty. Br J Ophthalmol 1999;83(3):327-333.

66. Anwar M, Teichmann KD. Deep lamellar keratoplasty: surgical techniques for anterior lamellar keratoplasty with and without baring of Descemet's membrane. Cornea 2002;21(4):374-383.

67. Mosca L, Fasciani R, Tamburelli C, et al. Femtosecond laser-assisted lamellar keratoplasty: early results. Cornea 2008;27(6):668-672.

68. Buzzonetti L, Laborante A, Petrocelli G. Standardized big-bubble technique in deep anterior lamellar keratoplasty assisted by the femtosecond laser. J Cataract Refract Surg 2010;36(10):1631-1636.

69. Scorcia V, Busin M, Lucisano A, Beltz J, Carta A, Scorcia G. Anterior Segment Optical Coherence Tomography-Guided Big-Bubble Technique. Ophthalmology 2013;120(3):471-476.

70. De Benito-Llopis L, Mehta JS, Angunawela RI, Ang M, Tan DT. Intraoperative anterior segment optical coherence tomography: a novel assessment tool during deep anterior lamellar keratoplasty. Am J Ophthalmol 2014;157(2):334-341 e333.

71. Muraine M, Toubeau D, Gueudry J, Brasseur G. Impact of new lamellar techniques of keratoplasty on eye bank activity. Graefes Arch Clin Exp Ophthalmol 2007;245(1):32-38.

72. Borderie VM, Sandali O, Bullet J, Gaujoux T, Touzeau O, Laroche L. Long-term results of deep anterior lamellar versus penetrating keratoplasty. Ophthalmology 2012;119(2):249-255.

73. Bahar I, Kaiserman I, Srinivasan S, Ya-Ping J, Slomovic AR, Rootman DS. Comparison of three different techniques of corneal transplantation for keratoconus. Am J Ophthalmol 2008;146(6):905-912 e901.

74. Funnell CL, Ball J, Noble BA. Comparative cohort study of the outcomes of deep lamellar keratoplasty and penetrating keratoplasty for keratoconus. Eye (Lond) 2006;20(5):527-532.

75. Reinhart WJ, Musch DC, Jacobs DS, Lee WB, Kaufman SC, Shtein RM. Deep anterior lamellar keratoplasty as an alternative to penetrating keratoplasty a report by the american academy of ophthalmology. Ophthalmology 2011;118(1):209-218.

76. Anshu A, Parthasarathy A, Mehta JS, Htoon HM, Tan DT. Outcomes of therapeutic deep lamellar keratoplasty and penetrating keratoplasty for advanced infectious keratitis: a comparative study. Ophthalmology 2009;116(4):615-623.

77. Panda A, Bageshwar LM, Ray M, Singh JP, Kumar A. Deep lamellar keratoplasty versus penetrating keratoplasty for corneal lesions. Cornea 1999;18(2):172-175.

78. Krumeich JH, Knulle A, Krumeich BM. [Deep anterior lamellar (DALK) vs. penetrating keratoplasty (PKP): a clinical and statistical analysis]. Klin Monbl Augenheilkd 2008;225(7):637-648.

79. Kawashima M, Kawakita T, Den S, Shimmura S, Tsubota K, Shimazaki J. Comparison of deep lamellar keratoplasty and penetrating keratoplasty for lattice and macular corneal dystrophies. Am J Ophthalmol 2006;142(2):304-309.

80. Silva CA, Schweitzer de Oliveira E, Souza de Sena Junior MP, Barbosa de Sousa L. Contrast sensitivity in deep anterior lamellar keratoplasty versus penetrating keratoplasty. Clinics (Sao Paulo) 2007;62(6):705-708.

81. Watson SL, Ramsay A, Dart JK, Bunce C, Craig E. Comparison of deep lamellar keratoplasty and penetrating keratoplasty in patients with keratoconus. Ophthalmology 2004;111(9):1676-1682.

82. Han DC, Mehta JS, Por YM, Htoon HM, Tan DT. Comparison of outcomes of lamellar keratoplasty and penetrating keratoplasty in keratoconus. Am J Ophthalmol 2009;148(5):744-751 e741. 
83. Cheng YY, Visser N, Schouten JS, et al. Endothelial cell loss and visual outcome of deep anterior lamellar keratoplasty versus penetrating keratoplasty: a randomized multicenter clinical trial. Ophthalmology 2011;118(2):302-309.

84. Ardjomand N, Hau S, McAlister JC, et al. Quality of vision and graft thickness in deep anterior lamellar and penetrating corneal allografts. Am J Ophthalmol 2007;143(2):228-235.

85. Alio JL. Visual improvement after late debridement of residual stroma after anterior lamellar keratoplasty. Cornea 2008;27(8):871-873.

86. Fontana L, Parente G, Sincich A, Tassinari G. Influence of graft-host interface on the quality of vision after deep anterior lamellar keratoplasty in patients with keratoconus. Cornea 2011;30(5):497-502.

87. Javadi MA, Feizi S, Yazdani S, Mirbabaee F. Deep anterior lamellar keratoplasty versus penetrating keratoplasty for keratoconus: a clinical trial. Cornea 2010;29(4):365-371.

88. Akdemir MO, Kandemir B, Sayman IB, Selvi C, Kamil Dogan O. Comparison of contrast sensitivity and visual acuity between deep anterior lamellar keratoplasty and penetrating keratoplasty in patients with keratoconus. International journal of ophthalmology 2012;5(6):737-741.

89. Baradaran-Rafii A, Eslani M, Sadoughi MM, Esfandiari H, Karimian F. Anwar versus Melles deep anterior lamellar keratoplasty for keratoconus: a prospective randomized clinical trial. Ophthalmology 2013;120(2):252-259.

90. Watson SL, Tuft SJ, Dart JK. Patterns of rejection after deep lamellar keratoplasty. Ophthalmology 2006;113(4):556-560.

91. Tillett CW. Posterior lamellar keratoplasty. Am J Ophthalmol 1956;41(3):530-533.

92. Melles GR, Eggink FA, Lander F, et al. A surgical technique for posterior lamellar keratoplasty. Cornea 1998;17(6):618-626.

93. Terry MA, Ousley PJ. Deep lamellar endothelial keratoplasty in the first United States patients: early clinical results. Cornea 2001;20(3):239-243.

94. Melles GR, Wijdh RH, Nieuwendaal CP. A technique to excise the descemet membrane from a recipient cornea (descemetorhexis). Cornea 2004;23(3):286-288.

95. Price FW, Jr., Price MO. Descemet's stripping with endothelial keratoplasty in 50 eyes: a refractive neutral corneal transplant. J Refract Surg 2005;21(4):339-345.

96. Gorovoy MS. Descemet-stripping automated endothelial keratoplasty. Cornea 2006;25(8):886-889.

97. Cheng YY, Pels E, Nuijts RM. Femtosecond-laser-assisted Descemet's stripping endothelial keratoplasty. J Cataract Refract Surg 2007;33(1):152-155.

98. Melles GR, Ong TS, Ververs B, van der Wees J. Descemet membrane endothelial keratoplasty (DMEK). Cornea 2006;25(8):987-990.

99. McCauley MB, Price FW, Jr., Price MO. Descemet membrane automated endothelial keratoplasty: hybrid technique combining DSAEK stability with DMEK visual results. J Cataract Refract Surg 2009;35(10):1659-1664.

100. Price MO, Price FW. Descemet's stripping endothelial keratoplasty. Curr Opin Ophthalmol 2007;18(4):290-294.

101. Price FW, Jr., Price MO. Endothelial keratoplasty to restore clarity to a failed penetrating graft. Cornea 2006;25(8):895-899.

102. Anshu A, Price MO, Tan DT, Price FW, Jr. Endothelial keratoplasty: a revolution in evolution. Surv Ophthalmol 2012;57(3):236-252.

103. Anshu A, Price MO, Price FW, Jr. Risk of corneal transplant rejection significantly reduced with Descemet's membrane endothelial keratoplasty. Ophthalmology 2012;119(3):536-540.

104. Busin M, Albe E. Does thickness matter: ultrathin Descemet stripping automated endothelial keratoplasty. Curr Opin Ophthalmol 2014;25(4):312-318. 
105. Terry MA, Ousley PJ. Deep lamellar endothelial keratoplasty visual acuity, astigmatism, and endothelial survival in a large prospective series. Ophthalmology 2005;112(9):1541-1548.

106. Price MO, Price FW, Jr. Descemet's stripping with endothelial keratoplasty: comparative outcomes with microkeratome-dissected and manually dissected donor tissue. Ophthalmology 2006;113(11):1936-1942.

107. Terry MA, Shamie N, Chen ES, Phillips PM, Hoar KL, Friend DJ. Precut tissue for Descemet's stripping automated endothelial keratoplasty: vision, astigmatism, and endothelial survival. Ophthalmology 2009;116(2):248-256.

108. Terry MA, Shamie N, Chen ES, et al. Endothelial keratoplasty for Fuchs' dystrophy with cataract: complications and clinical results with the new triple procedure. Ophthalmology 2009;116(4):631639.

109. Chen ES, Terry MA, Shamie N, Hoar KL, Friend DJ. Descemet-stripping automated endothelial keratoplasty: six-month results in a prospective study of 100 eyes. Cornea 2008;27(5):514-520.

110. Dirisamer M, Ham L, Dapena I, et al. Efficacy of descemet membrane endothelial keratoplasty: clinical outcome of 200 consecutive cases after a learning curve of 25 cases. Arch Ophthalmol 2011;129(11):1435-1443.

111. Tourtas T, Laaser K, Bachmann BO, Cursiefen C, Kruse FE. Descemet membrane endothelial keratoplasty versus descemet stripping automated endothelial keratoplasty. Am J Ophthalmol 2012;153(6):1082-1090 e1082.

112. Rodriguez-Calvo-de-Mora M, Quilendrino R, Ham L, et al. Clinical outcome of 500 consecutive cases undergoing Descemet's membrane endothelial keratoplasty. Ophthalmology 2015;122(3):464470.

113. Price MO, Giebel AW, Fairchild KM, Price FW, Jr. Descemet's membrane endothelial keratoplasty: prospective multicenter study of visual and refractive outcomes and endothelial survival. Ophthalmology 2009;116(12):2361-2368.

114. Ham L, Dapena I, van Luijk C, van der Wees J, Melles GR. Descemet membrane endothelial keratoplasty (DMEK) for Fuchs endothelial dystrophy: review of the first 50 consecutive cases. Eye (Lond) 2009;23(10):1990-1998.

115. Khor WB, Mehta JS, Tan DT. Descemet stripping automated endothelial keratoplasty with a graft insertion device: surgical technique and early clinical results. Am J Ophthalmol 2011;151(2):223232 e222.

116. Koenig SB, Covert DJ, Dupps WJ, Jr., Meisler DM. Visual acuity, refractive error, and endothelial cell density six months after Descemet stripping and automated endothelial keratoplasty (DSAEK). Cornea 2007;26(6):670-674.

117. Price MO, Price FW, Jr. Endothelial cell loss after descemet stripping with endothelial keratoplasty influencing factors and 2-year trend. Ophthalmology 2008;115(5):857-865.

118. Li JY, Terry MA, Goshe J, Shamie N, Davis-Boozer D. Graft rejection after Descemet's stripping automated endothelial keratoplasty: graft survival and endothelial cell loss. Ophthalmology 2012;119(1):90-94.

119. Busin M, Madi S, Santorum P, Scorcia V, Beltz J. Ultrathin descemet's stripping automated endothelial keratoplasty with the microkeratome double-pass technique: two-year outcomes. Ophthalmology 2013;120(6):1186-1194.

120. Terry MA, Straiko MD, Goshe JM, et al. Endothelial Keratoplasty: Prospective, Randomized, Masked Clinical Trial Comparing an Injector With Forceps for Tissue Insertion. Am J Ophthalmol 2013. 
121. Price MO, Gorovoy M, Price FW, Jr., Benetz BA, Menegay HJ, Lass JH. Descemet's stripping automated endothelial keratoplasty: three-year graft and endothelial cell survival compared with penetrating keratoplasty. Ophthalmology 2013;120(2):246-251.

122. Price MO, Fairchild KM, Price DA, Price FW, Jr. Descemet's stripping endothelial keratoplasty fiveyear graft survival and endothelial cell loss. Ophthalmology 2011;118(4):725-729.

123. Goldich Y, Showail M, Avni-Zauberman N, et al. Contralateral eye comparison of descemet membrane endothelial keratoplasty and descemet stripping automated endothelial keratoplasty. Am J Ophthalmol 2015;159(1):155-159 e151.

124. Feng MT, Price MO, Miller JM, Price FW, Jr. Air reinjection and endothelial cell density in Descemet membrane endothelial keratoplasty: five-year follow-up. J Cataract Refract Surg 2014;40(7):11161121.

125. Baydoun L, Tong CM, Tse WW, et al. Endothelial cell density after descemet membrane endothelial keratoplasty: 1 to 5-year follow-up. Am J Ophthalmol 2012;154(4):762-763.

126. Lee WB, Jacobs DS, Musch DC, Kaufman SC, Reinhart WJ, Shtein RM. Descemet's stripping endothelial keratoplasty: safety and outcomes: a report by the American Academy of Ophthalmology. Ophthalmology 2009;116(9):1818-1830.

127. Price FW, Jr., Price MO. Descemet's stripping with endothelial keratoplasty in 200 eyes: Early challenges and techniques to enhance donor adherence. J Cataract Refract Surg 2006;32(3):411-418.

128. Terry MA, Hoar KL, Wall J, Ousley P. Histology of dislocations in endothelial keratoplasty (DSEK and DLEK): a laboratory-based, surgical solution to dislocation in 100 consecutive DSEK cases. Cornea 2006;25(8):926-932.

129. Patel SV. Graft survival and endothelial outcomes in the new era of endothelial keratoplasty. Experimental eye research 2012;95(1):40-47.

130. Terry MA, Shamie N, Chen ES, Hoar KL, Friend DJ. Endothelial keratoplasty a simplified technique to minimize graft dislocation, iatrogenic graft failure, and pupillary block. Ophthalmology 2008;115(7):1179-1186.

131. Suh LH, Yoo SH, Deobhakta A, et al. Complications of Descemet's stripping with automated endothelial keratoplasty: survey of 118 eyes at One Institute. Ophthalmology 2008;115(9):1517-1524.

132. Cheng $Y Y$, Hendrikse F, Pels $E$, et al. Preliminary results of femtosecond laser-assisted descemet stripping endothelial keratoplasty. Arch Ophthalmol 2008;126(10):1351-1356. 



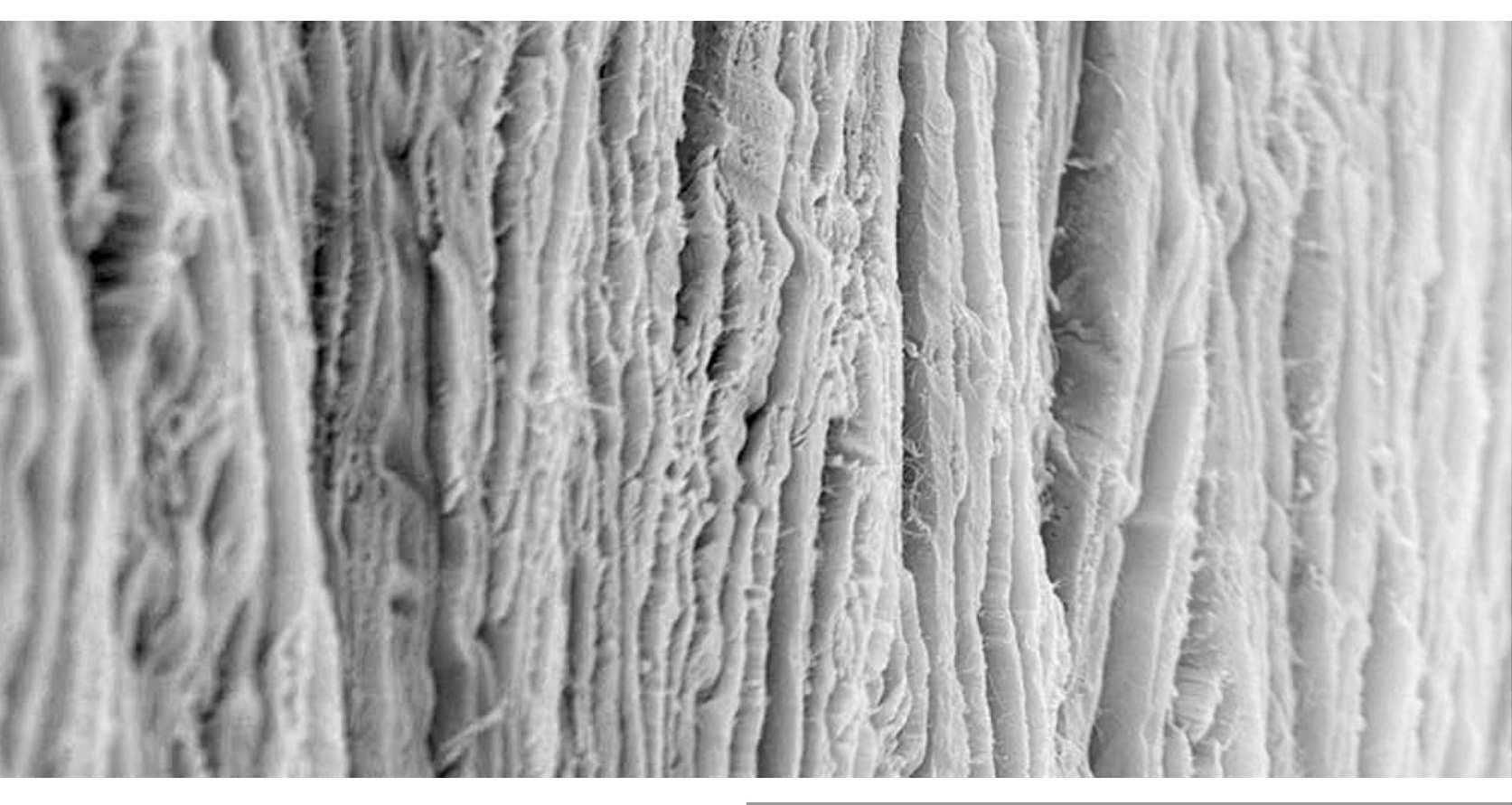




\title{
CHAPTER 2
}

Histologic Evaluation of Human

Posterior Lamellar Discs for

Femtosecond Laser Descemet's

Stripping Endothelial Keratoplasty

\author{
Yanny Y.Y. Cheng \\ Shin J. Kang \\ Hans E. Grossniklaus \\ Elisabeth Pels \\ Hans J.Q. Duimel \\ Peter M. Frederik \\ Fred Hendrikse \\ Rudy M.M.A. Nuijts
}

Cornea. 2009 Jan;28(1):73-79 


\section{ABSTRACT}

Purpose: To evaluate the histologic changes in corneal structure after femtosecond laser preparation of posterior lamellar discs, more specifically, the smoothness of the stromal bed and the accuracy of the predicted depth of the horizontal lamellar cut.

Materials and methods: Nineteen human donor eyes unsuitable for transplantation were used. Femtosecond laser was used to prepare a horizontal lamellar cut in donor corneas at a depth of $400 \mu \mathrm{m}$. Transmission electron microscopy images were used to evaluate the changes in the corneal structure and to measure the damage zone. Scanning electron microscopy images were used to determine the relative depth of the horizontal lamellar cut, and the stromal bed was examined to determine the smoothness of the surface.

Results: Transmission electron microscopy images showed a mean damage zone of 6.8 $\pm 3.1 \mu \mathrm{m}$, which consisted of irregularly oriented collagen fibrils and electron-dense granular material. The collagen lamellae, both anteriorly and posteriorly of the damaged zone, showed a regular parallel configuration. The relative depth of the horizontal lamellar cut as percentage of the total corneal thickness in the center and periphery were $70.4 \pm 4.5 \%$ and $55.6 \pm 5.9 \%$. Scanning electron microscopy images of the stromal bed showed a relatively smooth surface.

Conclusion: The femtosecond laser is effective to prepare a deep horizontal lamellar cut in a standardized method. The stromal bed is smooth and without extensive adjacent tissue damage. The posterior lamellar disc is thinner in the center and thicker at the edges, which may produce a mild hyperopic shift after femtosecond laser-assisted Descemet's stripping endothelial keratoplasty. 


\section{INTRODUCTION}

Corneal endothelial disease has traditionally been treated with penetrating keratoplasty, but in recent years posterior lamellar keratoplasty or deep lamellar endothelial keratoplasty (DLEK) surgical techniques have become increasingly popular. These surgical techniques are designed to replace the diseased corneal endothelial layer without manipulation of the healthy anterior part of the recipient cornea in pseudophakic or aphakic bullous keratopathy and in Fuchs endothelial dystrophy. ${ }^{1,2}$

Posterior lamellar keratoplasty and DLEK were not widely practiced due to the technical challenges, but this has been changed after introduction of Descemet's stripping endothelial keratoplasty (DSEK) and Descemet's stripping automated endothelial keratoplasty (DSAEK)..$^{3-7}$ DSAEK allows to remove the dysfunctional corneal endothelium in the recipient eye in a standardized fashion and to prepare the posterior lamellar disc (PLD) in a standardized method by using an artificial anterior chamber system with a mechanical microkeratome.

We performed a study to examine the feasibility of a femtosecond laser to prepare a PLD in human donor corneas for a DSEK procedure.We evaluated the changes in the corneal structure, the accuracy of the predicted depth of a horizontal lamellar cut in swollen corneas of whole eyes, and the smoothness of the stromal bed.

\section{MATERIALS \& METHODS}

\section{Donor tissue}

Nineteen human donor eyes unsuitable for transplantation because of corneal scarring $(n=18)$ and pterygium ( $n=1)$, were obtained from the Cornea Bank Amsterdam (Netherlands Institute for Neuroscience, Amsterdam, The Netherlands) and transported to the University Hospital Maastricht (Maastricht, The Netherlands) in a moist chamber at $4^{\circ} \mathrm{C}$.

\section{Femtosecond laser surgical procedure}

The eyes were immobilized using an eye holder with a suction ring (D.O.R.C. International, Zuidland, The Netherlands). Slit-lamp microscopy and corneal thickness measurements (Orbtek Orbscan II, version 3.10.31, Bausch \& Lomb, Munich, Germany) were performed before the femtosecond laser procedure (AMO-Intralase Corp., Irvine, CA). The epithelium was removed with a cellulose spear and a surgical beaver blade. The pressure was measured using a Barraquer tonometer (Bausch and Lomb, Munich, Germany). The intraocular pressure was maintained between a range of $40 \mathrm{mmHg}$ and $60 \mathrm{mmHg}$. A deep horizontal lamellar cut in the cornea was prepared using a $30-\mathrm{kHz}$ femtosecond 
laser. The cornea was applanated with a flat interface lens. The horizontal lamellar cut was set at a depth of $400 \mu \mathrm{m}$ with a diameter of $9.5 \mathrm{~mm}$ and the mean energy level was $1.07 \mu \mathrm{J}$ (range $0.70-1.40 \mu \mathrm{J}$ ) using a raster pattern. After the femtosecond laser procedure the whole donor eyes were decontaminated and stored in organ culture for two weeks in the Cornea Bank Amsterdam. After organ culture storage, 6 corneoscleral buttons with a horizontal lamellar cut (without dissection of the PLD) were fixed in $2.5 \%$ glutaraldehyde in $0.1 \mathrm{M}$ phosphate buffer or $4 \%$ formalin for histologic and electron microscopic examination. Additionally, 13 corneoscleral buttons with a horizontal lamellar cut were punched from the endothelial side with an 8.0- $\mathrm{mm}$ trephine (Medical Workshop, Groningen, the Netherlands). The PLD was separated from the anterior stroma by injection of sterile balanced salt solution in the horizontal lamellar interface to create an intralamellar dissection plane. After dissection of the PLD all specimens were fixed in $2.5 \%$ glutaraldehyde in $0.1 \mathrm{M}$ phosphate buffer or $4 \%$ formalin for histologic and electron microscopic examination.

\section{Histology and electron microscopy}

For transmission electron microscopy (TEM), the corneas were postfixed in $1 \%$ osmium tetroxide, en bloc stained with $2 \%$ uranyl acetate, serially dehydrated in graded ethanols followed by a 1:1 mixture of $100 \%$ ethanol and propylene oxide overnight. They were transferred into pure LX-112 resin (Ladd Research Industries, Vt) and after several hours were oriented in silicone molds with fresh resin and cured at $50^{\circ} \mathrm{C}$ for 2 days. Resin blocks were trimmed, $1.5-\mu \mathrm{m}$-thick sections were cut and stained with $1 \%$ toluidine blue to determine the area of interest for thin sectioning and for light microscopy. Ultrathin sections $(80 \mathrm{~nm}$ ) were cut with a Diatome diamond knife on a Leica Ultracut ultramicrotome and picked up on uncoated 300 mesh grids. Sections were stained with uranyl acetate and lead citrate and viewed in a Jeol 100CS-II TEM at $60 \mathrm{kv}$. Images were captured on Kodak EM film \#4489 and developed in Kodak D-19.

TEM images of non-dissected corneoscleral buttons were used to evaluate the corneal structure, anteriorly and posteriorly of the horizontal lamellar cut, and to measure the extent of corneal stromal damage induced by the femtosecond laser. The measurements were performed using the ImageJ 1.35s software (available at http://rsb.info.nih.gov/ij; developed by Wayne Rasband, National Institute of Health, Bethesda, MD).

For scanning electron microscopy (SEM), the corneas were dehydrated, critical point dried, mounted on specimen tubs, and coated with gold. SEM images of corneoscleral buttons were used to measure the relative depth of the horizontal lamellar cut. The corneoscleral buttons were hemi-dissected in a anterior-posterior direction from epithelium to endothelium. SEM images were made facing the corneal stromal surface in the epithelial-endothelial direction. Analysis of the relative depth of the horizontal lamellar cut in the cornea was performed using the ImageJ 1.35 s software. 
The anterior lamellar discs and the PLDs of the dissected corneoscleral buttons were processed to evaluate the smoothness of the stromal side of the discs. The surface of the anterior lamellar discs and posterior lamellar discs were examined with SEM (Philips XL 30 Scanning Electron Microscopy, Eindhoven, Netherlands) at 10 kV. SEM images were subjectively graded by 3 masked independent observers. The smoothness of the stromal bed of the discs were graded as follows: 1 = very smooth, $2=$ smooth, $3=$ rough; and $4=$ very rough.

\section{Statistical analysis}

The difference of smoothness between the anterior lamellar disc and the PLD was compared by the Wilcoxon matched-pair test. A $P$-value of $\leq 0.05$ was considered statistically significant. All data analysis was performed using the SPSS statistical software package (SPSS for Windows version 12.0, SPSS Inc., Chicago, IL).

\section{RESULTS}

\section{Corneal stroma collagen and keratocytes}

On light microscopy, there was a smooth, straight horizontal lamellar cut in the cornea (Fig. 1).TEM images in the same area showed a mean damage zone of $6.8 \pm 3.1 \mu \mathrm{m}$ (range, 2.2-14.3 $\mu \mathrm{m}$ ), which consisted of irregularly oriented collagen fibrils, and electron dense

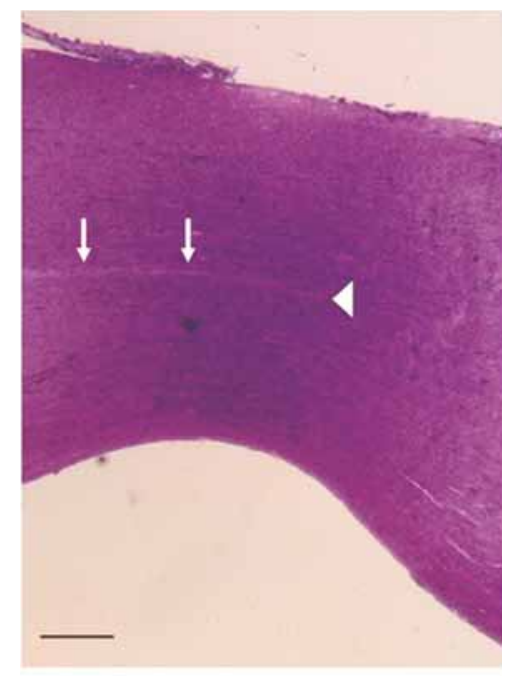

Figure 1. Light microscopic, cross-sectional histology (toluidine blue) of the corneoscleral rim with a horizontal lameller cut. The toluidine blue sections of the corneoscleral rim showed a horizontal lamellar cut (arrows) in a human cornea using the femtosecond laser. The large arrowhead showed the end of the horizontal lamellar cut at the corneal periphery. Scale bar, $100 \mu \mathrm{m}$. 

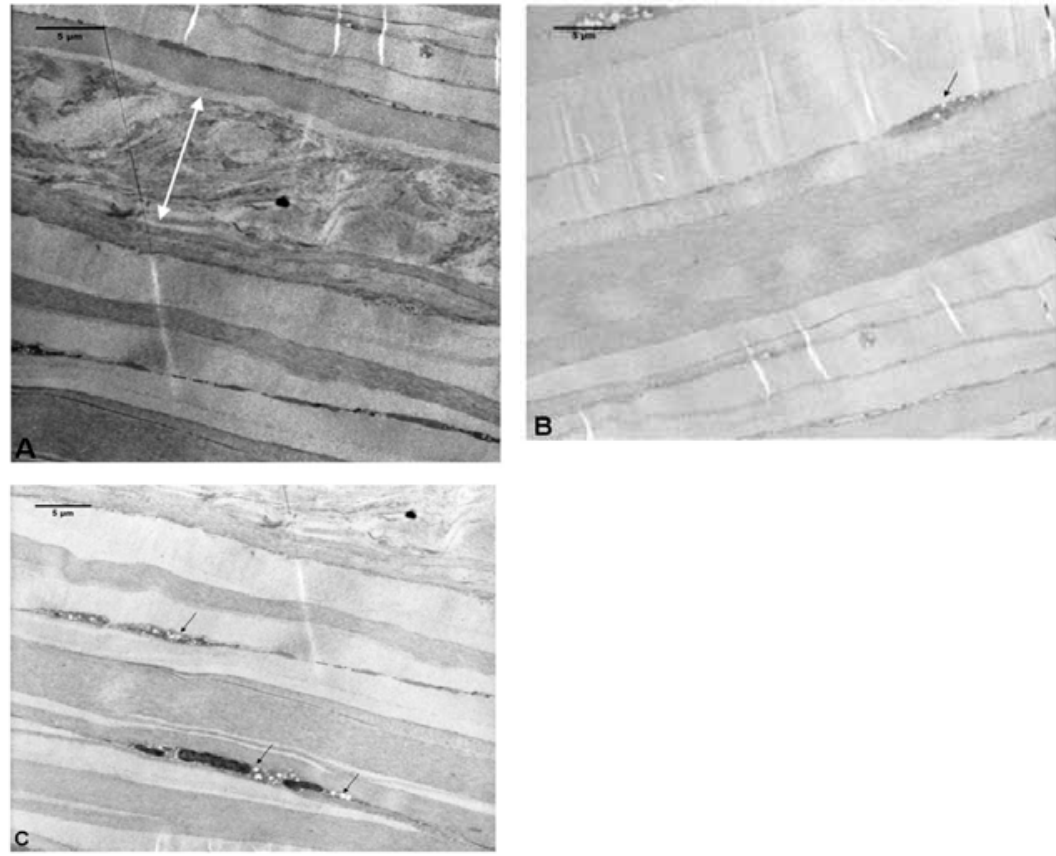

Figure 2. Transmission electron micrographs of the cornea after horizontal lamellar cut using the $30-\mathrm{kHz}$ femtosecond laser. Transmission electron micrograph showed a mean damage area of $6.8 \pm 3.1 \mu \mathrm{m}$ (A, between arrows). The collagen lamellae above (B) and below (C) the horizontal lamellar cut showed a regular parallel-oriented configuration and. After 2 weeks organ culture storage with Dextran, numerous vacuoles were detected in the keratocytes ( $B$ and $C$, arrows)

granular material (Fig. 2A). The collagen lamellae, both anteriorly and posteriorly of the damaged zone, showed a regular parallel lamellar configuration (Fig. 2B, 2C). Thermal alteration of adjacent tissue was not detected with light microscopy and TEM. The keratocytes found just adjacent to the damage zone, both anteriorly and posteriorly, contained numerous vacuoles and looked similar to keratocytes after corneal storage with 5\% Dextran T500 (Pharmacia, Amsterdam, The Netherlands) supplement.

\section{Depth of the horizontal lamellar cut}

The mean central corneal thickness and peripheral corneal thickness ( $4 \mathrm{~mm}$ from the center) before the femtosecond laser procedure was $820.0 \pm 51.2 \mu \mathrm{m}$ and $951.5 \pm 51.5$ $\mu \mathrm{m}$, respectively. On light microscopy and SEM, the mean relative depth of the horizontal lamellar cut as percentage of the total corneal thickness in the center and periphery were $66.4 \% \pm 4.3 \%$ versus $57.6 \% \pm 3.0 \%$ (Fig. 3 ), and $70.4 \% \pm 4.5 \%$ versus $55.6 \% \pm 5.9 \%$, respectively (Fig. 4). 


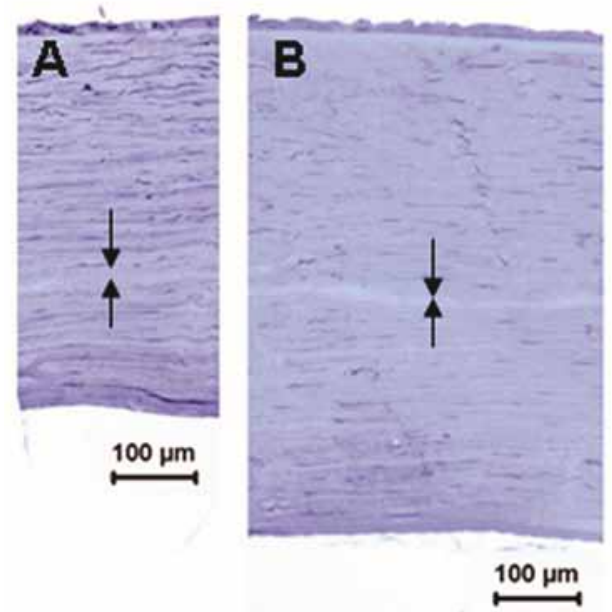

Figure 3. Light microscopic, cross-sectional histology of the cornea in the center and in the peripheral (toluidine blue). The toluidine blue sections showed that the horizontal lamellar cut was located at $66.4 \%$ of the total corneal thickness in the center ( $\mathrm{A}$, between arrows), and $57.6 \%$ of the total corneal thickness in the peripheral ( $\mathrm{B}$, between arrows).
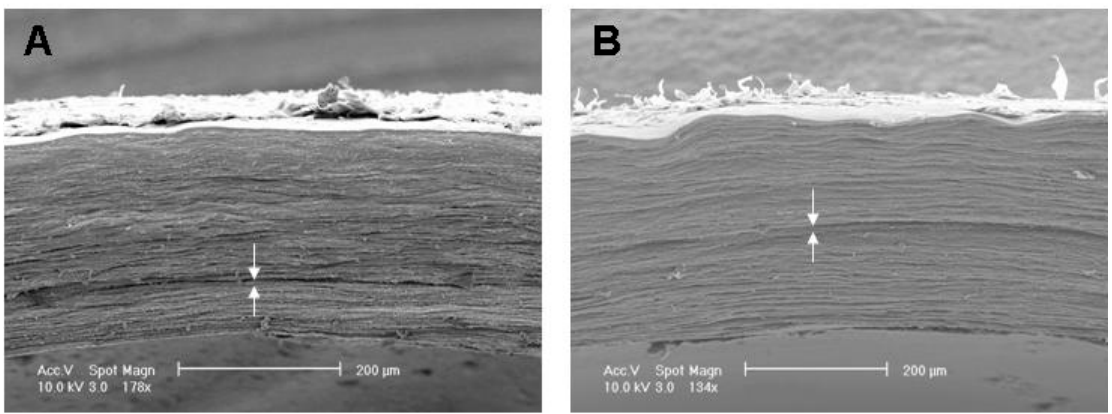

Figure 4. Scanning electron micrographs of human cornea showing the horizontal lamellar cut. Scanning electron micrographs showed in the cornea a horizontal lamellar cut produced with the $30-\mathrm{kHz}$ femtosecond laser. In the center of the cornea, the mean relative depth of the horizontal lamellar cut is located at $70.4 \%$ of the total corneal thickness $(A$, between arrows), and in the periphery it is located at $55.6 \%$ of the total corneal thickness (B, between arrows).

\section{Quality of the stromal bed}

After 2 weeks in organ culture storage, the cornea was trephined and all PLDs were separated by blunt dissection. The histology of the anterior lamellar disc and the PLD demonstrated a relatively smooth surface. Figure 5 shows the SEM images of the four gradings of smoothness. The mean smoothness score of SEM for the stromal bed of the anterior lamellar disc and PLD was $1.5 \pm 0.4$ and $2.8 \pm 0.9$, respectively $(P=0.027$; Fig. 6). 

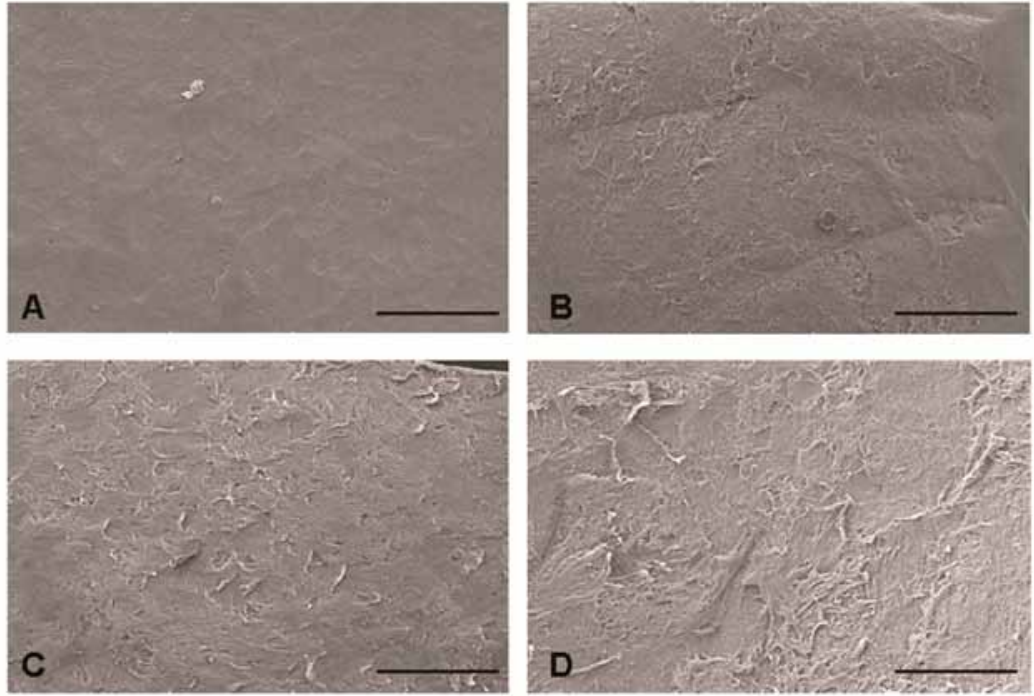

Figure 5. The four gradings of the stromal smoothness. SEM images showed a very smooth stromal bed (A), smooth stromal bed (B), rough stromal bed (C), and very rough stromal bed (D). Scale bar, $500 \mu \mathrm{m}$.
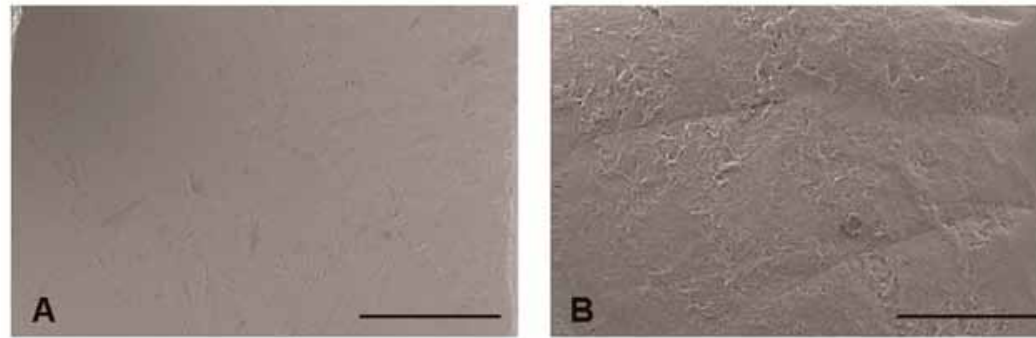

Figure 6. Scanning electron micrographs of the stromal bed after dissection. Two representative SEM images for the average smoothness of the anterior lamellar disc ( 1.3 score in the this case; $A$ ) and the PLD ( 2.7 score in this case; B) are shown. Scale bar, $500 \mu \mathrm{m}$.

\section{DISCUSSION}

The introduction of more standardized techniques like DSEK and DSAEK have led to renewed interest in endothelial keratoplasty for replacement of the diseased corneal endothelial layer and to a dramatic increase in the percentage of donor tissue used for endothelial keratoplasty. ${ }^{4,8,9}$ Several in vivo and in vitro studies have described different techniques to prepare a PLD from whole donor eyes or corneoscleral buttons using mechanical microkeratomes or femtosecond lasers. ${ }^{5,710-14}$

Currently, there are three femtosecond lasers available for corneal surgical applications: Intralase (AMO-Intralase Corp.), Femtec (20/10 Perfect Vision, Heidelberg, Germany), 
and Ziemer Ophthalmic Systems AG (FEMTO LDV, Port, Switzerland). Experimental and clinical applications of femtosecond lasers include laser in situ keratomileusis (LASIK) flap creation, ${ }^{15,16}$ tunnel incision for corneal rings, ${ }^{17,18}$ wedge-shaped resection in correction of high residual astigmatism, ${ }^{19}$ lamellar and full-thickness keratoplasty techniques $^{7,10,12,14,20-22}$ and shaped full-thickness keratoplasty with different donor-recipient patterns. ${ }^{23-27}$ Recently, the feasiblity of the femtosecond laser for preparing PLDs for DSEK was described. ${ }^{14}$

The challenge for all lamellar keratoplasty procedures including endothelial keratoplasty is to achieve a high level of postoperative quality of vision. It is suggested that the lack of stromal smoothness of the PLDs is a major factor in the pathogenesis of interface opacification that may be responsible for some loss of the potential best-corrected visual acuity. ${ }^{28}$ Although the stromal bed of the recipient cornea after Descemet's stripping is very smooth, the stromal bed of the PLD prepared by either manual dissection or by an automated microkeratome is not as smooth as the bed of the recipient. ${ }^{29}$ Several studies have shown that a femtosecond laser and automated microkeratome are effective in preparing a PLD, but the smoothness of the stromal bed of a deep horizontal lamellar cut appears to be inferior to the smoothness of the stromal bed of a superficial LASIK flap cut by a femtosecond laser., ${ }^{710-13}$

Recently, the smoothness of the corneal cuts made by a femtosecond laser have been related to the type of laser and laser pattern used, the frequency and energy of the laser and the type of applanation lens, being flat or curved. When using the FEMTEC femtosecond laser, a smooth stromal bed with minor remaining tissue bridges of PLDs may be visible ${ }^{10}$ and after full-thickness trephination with this laser electron microscopy reveals more tissue bridges in thicker edematous corneas than in thinner corneas. ${ }^{21}$ PLDs prepared by the Intralase femtosecond laser showed an excellent side-cut quality and a smooth lamellar bed. ${ }^{12}$ However, when using a spiral laser pattern SEM showed a stromal bed with very mild concentric circular ridges and a stucco-like texture. Sarayba et $\mathrm{al}^{30}$ further evaluated the quality of the stromal bed using a spiral or raster pattern and demonstrated that a raster pattern provides a smoother stromal bed than a spiral pattern. It has been suggested that the circular concentric circular ridges will disappear by the application of a curved interface ${ }^{12}$ and that lower energy levels may result in a smoother stromal bed. Indeed, for superficial LASIK flaps it has been shown that a 30$\mathrm{kHz}$ femtosecond laser with tighter spot/line separation and lower energy level creates a smoother stromal bed in comparison with a $15-\mathrm{kHz}$ femtosecond laser and a mechanical microkeratome. ${ }^{31}$ For the moment it is not clear what the effect of higher frequency femtosecond lasers up to $60 \mathrm{kHz}$ on the smoothness of the stromal bed may be when making deeper stromal lamellar cuts for PLDs. The effect of the hydrated corneas (mean corneal thickness $820 \mu \mathrm{m}$ before the horizontal lamellar cut) on the smoothness of the cut is not clear. One could speculate that posterior interface cuts tend to be more irregu- 
lar because of a looser stromal fibrillar arrangement and density and more laser scatter from going through more tissue. However, in an environment of extensive hydration as in these fresh donor eyes, the number of fibrils cut by the laser per square millimeter is lower. It is unclear if this might result in less roughness at the interface as compared with cutting in a normally hydrated posterior corneal stroma.

In the present study, we have used a raster pattern instead of a spiral pattern, and concentric circular ridges were not seen in the SEM photographs. We found that the smoothness of the stromal bed of the PLD appears to be comparable to the results published by Terry et al. ${ }^{11}$ However, the stromal bed of the anterior lamellar disc appears significantly smoother as compared to the stromal bed of the PLD of the same donor. At present we have no explanantion for this finding. Trephination side cuts were not made by the femtosecond laser because the maximum cutting depth of the femtosecond laser was $400 \mu \mathrm{m}$ at the time of the study, and we could not exclude that epithelial cells can migrate into the trephination side cuts during the 2 weeks of organ culture. In addition, we believe that the dissection of the PLD form the anterior part will be more traumatic for the endothelial cells when we need to manually dissect along the trephination side cuts. Further experiments are needed to study these hypotheses.

Recent reports showed that predictability of flap thickness after a superficial LASIK flap was significantly better when using a femtosecond laser [standard deviation (SD) of $14 \mu \mathrm{m}$ ] as compared to the Carriazo-Barraquer (Moria, Inc., Antony, France) or Hansatome microkeratome (Baush \& Lomb, Inc.) (SD of 26 and $29 \mu \mathrm{m}$, respectively). ${ }^{16,32}$ In vitro studies in cloudy and edematous corneas showed a mean SD between 9 and 61 $\mu \mathrm{m}$ from the intended thickness for PLDs prepared by a femtosecond laser. ${ }^{12,30}$ When using a mechanical microkeratome with a 350- $\mu \mathrm{m}$ microkeratome head, the SD of the anterior lamellar disc was within $\pm 75 \mu \mathrm{m} .{ }^{33}$ Behrens et al ${ }^{34}$ showed that the accuracy of the intended thickness of the anterior lamellar discs decreased, when the head thickness of the automated microkeratome increased. When the intra chamber pressure was increased the mean SD of the thickness of the anterior lamellar discs decreased. ${ }^{35}$

In our study the PLD was not dissected immediately after the femtosecond laser procedure, so we could only measure the relative thickness of the posterior lamellar disc after the histologic fixation procedures. Both light microscopy and SEM revealed that the horizontal lamellar cut was deeper in the center than in the periphery. This means that the Intralase femtosecond laser will provide a meniscus-shaped PLD that is thinner in the center and thicker at the edges. A meniscus-shaped PLD can produce a mild hyperopic shift, as has been shown in a previous study. ${ }^{36}$ With the current laser technology, the depth of the horizontal lamellar cut is calculated from the anterior surface of the donor cornea. When it is possible to calculate the depth of the horizontal lamellar cut from the endothelial side of the cornea, the PLD will have a more planar shape, which may avoid the hyperopic shift. 
Obviously, during the fixation procedure for electron microscopy the corneas were dehydrated, which could result in a shift of the level of the horizontal lamellar cut, that is, the ratio of stroma anteriorly versus posteriorly of the lamellar cut as compared with total corneal thickness. We cannot exclude that these factors could lead to an inaccuracy of the measurements of the depth of the horizontal lamellar cut. In our study an area of loose debris of the collagen fibrils of the order of $6.8 \pm 3.1 \mu \mathrm{m}$ was detected with TEM. The collagen lamellae above and below the damaged area had a normal ultrastructure. This indicates that the femtosecond laser produced a small area of disruption of collagen fibrils without adjacent thermal damage. However, other in vitro studies showed no thermal damage in the adjacent tissue when performing corneal trephination with the femtosecond laser. ${ }^{10,21}$ Keratocyte activation in a LASIK flap prepared with the microkeratome or femtosecond laser and after photorefractive keratectomy has been detected. ${ }^{37-40}$ After DLEK, activated keratocytes have been found just adjacent to the interface line. ${ }^{41}$ In vivo, a correlation between keratocyte activation and clinical corneal haze has been detected using confocal microscopy. ${ }^{39,40,42}$ Hindman et al $^{43}$ have used a scatterometer and found a higher scattering index in a DLEK group, which indicates an increased subclinical corneal haze that may limit best-corrected visual acuity after DLEK. It has been suggested that lower energy and smaller step size when using a raster pattern will minimize keratocyte activation and the inadvertent sequelae like interface opacification. ${ }^{44}$

In the present study the femtosecond laser is effective in creating a deep horizontal lamellar cut in a swollen cornea from a whole donor eye. Corneas with corneal scarring and healthy endothelial cells can now be predissected and used for endothelial keratoplasty, which may lead to more donor corneas available for grafting. We have found that preparing of the PLD with a femtosecond laser does not result in a significant amount of endothelial cell loss. ${ }^{14}$ The advantage of this procedure is that corneas with a femtosecond laser prepared horizontal lamellar can be stored in organ culture for up to 2 weeks before corneal transplantation. This allows time for serological screening of the donor, microbiological testing and human leucocyte antigen-typing of the tissue, and for quality assessment of the endothelial cel layer. ${ }^{45}$ In addition, precutting the tissue with a femtosecond laser could have cost-effective advantages. In contrary, when the PLD is cut in the surgical theatre by a microkeratome, no information is available on the endothelial status after the preparation.

In summary, femtosecond laser-assisted preparing of PLDs results in a standardized method of preparing PLD with a smooth surface and without extensive adjacent tissue damage. In a previous report we have shown that femtosecond laser-assisted DSEK in a patient with pseudophakic bullous keratoplasty is feasible. ${ }^{46} \mathrm{~A}$ multicenter randomized clinical study is in progress to compare the visual results and endothelial cell density 
of femtosecond laser-assisted DSEK with the results of the conventional penetrating keratoplasty. 


\section{REFERENCES}

1. Melles GR, Lander F, Beekhuis WH, et al. Posterior lamellar keratoplasty for a case of pseudophakic bullous keratopathy. Am J Ophthalmol 1999;127:340-341.

2. Terry MA, Ousley PJ. Deep lamellar endothelial keratoplasty in the first United States patients: early clinical results. Cornea 2001;20:239-243.

3. Price FW, Jr., Price MO. Descemet's stripping with endothelial keratoplasty in 200 eyes: Early challenges and techniques to enhance donor adherence. J Cataract Refract Surg 2006;32:411-418.

4. Gorovoy MS. Descemet-stripping automated endothelial keratoplasty. Cornea 2006;25:886-889.

5. Melles GR, Eggink FA, Lander F, et al. A surgical technique for posterior lamellar keratoplasty. Cornea 1998;17:618-626.

6. Terry MA, Ousley PJ. Replacing the endothelium without corneal surface incisions or sutures: the first United States clinical series using the deep lamellar endothelial keratoplasty procedure. Ophthalmology 2003;110:755-764; discussion 764.

7. Suwan-Apichon O, Reyes JM, Griffin NB, et al. Microkeratome Versus Femtosecond Laser Predissection of Corneal Grafts for Anterior and Posterior Lamellar Keratoplasty. Cornea 2006;25:966-968.

8. Price MO, Price FW. Descemet's stripping endothelial keratoplasty. Curr Opin Ophthalmol 2007; 18:290-294.

9. Tan DT, Mehta JS. Future directions in lamellar corneal transplantation. Cornea 2007;26:S21-28.

10. Seitz B, Langenbucher A, Hofmann-Rummelt C, et al. Nonmechanical posterior lamellar keratoplasty using the femtosecond laser (femto-plak) for corneal endothelial decompensation. Am J Ophthalmol 2003;136:769-772.

11. Terry MA, Ousley PJ, Will B. A practical femtosecond laser procedure for DLEK endothelial transplantation: cadaver eye histology and topography. Cornea 2005;24:453-459.

12. Soong HK, Mian S, Abbasi O, et al. Femtosecond laser-assisted posterior lamellar keratoplasty: initial studies of surgical technique in eye bank eyes. Ophthalmology 2005;112:44-49.

13. Sarayba MA, Juhasz T, Chuck RS, et al. Femtosecond laser posterior lamellar keratoplasty: a laboratory model. Cornea 2005;24:328-333.

14. Cheng YY, Pels E, Cleutjens JP, et al. Corneal endothelial viability after femtosecond laser preparation of posterior lamellar discs for Descemet-stripping endothelial keratoplasty. Cornea 2007;26:1118-1122.

15. Durrie DS, Stahl J. Randomized comparison of custom laser in situ keratomileusis with the Alcon CustomCornea and the Bausch \& Lomb Zyoptix systems: one-month results. J Refract Surg 2004;20:S614-618.

16. Patel SV, Maguire LJ, McLaren JW, et al. Femtosecond laser versus mechanical microkeratome for LASIK: a randomized controlled study. Ophthalmology 2007;114:1482-1490.

17. Rabinowitz YS, Li X, Ignacio TS, et al. INTACS inserts using the femtosecond laser compared to the mechanical spreader in the treatment of keratoconus. J Refract Surg 2006;22:764-771.

18. Ertan A, Kamburoglu G, Bahadir M. Intacs insertion with the femtosecond laser for the management of keratoconus: one-year results. J Cataract Refract Surg 2006;32:2039-2042.

19. Ghanem RC, Azar DT. Femtosecond-laser arcuate wedge-shaped resection to correct high residual astigmatism after penetrating keratoplasty. J Cataract Refract Surg 2006;32:1415-1419.

20. Mian SI, Soong HK, Patel SV, et al. In vivo femtosecond laser-assisted posterior lamellar keratoplasty in rabbits. Cornea 2006;25:1205-1209. 
21. Meltendorf C, Schroeter J, Bug R, et al. Corneal trephination with the femtosecond laser. Cornea 2006;25:1090-1092.

22. Holzer MP, Rabsilber TM, Auffarth GU. Penetrating keratoplasty using femtosecond laser. Am J Ophthalmol 2007;143:524-526.

23. Seitz B, Brunner H, Viestenz A, et al. Inverse mushroom-shaped nonmechanical penetrating keratoplasty using a femtosecond laser. Am J Ophthalmol 2005;139:941-944.

24. Ignacio TS, Nguyen TB, Chuck RS, et al. Top hat wound configuration for penetrating keratoplasty using the femtosecond laser: a laboratory model. Cornea 2006;25:336-340.

25. Steinert RF, Ignacio TS, Sarayba MA. "Top hat"-shaped penetrating keratoplasty using the femtosecond laser. Am J Ophthalmol 2007;143:689-691.

26. Buratto L, Bohm E. The use of the femtosecond laser in penetrating keratoplasty. Am J Ophthalmol 2007;143:737-742.

27. Farid M, Kim M, Steinert RF. Results of penetrating keratoplasty performed with a femtosecond laser zigzag incision initial report. Ophthalmology 2007;114:2208-2212.

28. Rich LF. Expanding the scope of lamellar keratoplasty. Trans Am Ophthalmol Soc 1999;97:771-814.

29. Terry MA, Hoar KL, Wall J, et al. Histology of dislocations in endothelial keratoplasty (DSEK and DLEK): a laboratory-based, surgical solution to dislocation in 100 consecutive DSEK cases. Cornea 2006;25:926-932.

30. Sarayba MA, Maguen E, Salz J, et al. Femtosecond laser keratome creation of partial thickness donor corneal buttons for lamellar keratoplasty. J Refract Surg 2007;23:58-65.

31. Sarayba MA, Ignacio TS, Binder PS, et al. Comparative study of stromal bed quality by using mechanical, IntraLase femtosecond laser 15- and 30-kHz microkeratomes. Cornea 2007;26:446-451.

32. Kezirian GM, Stonecipher KG. Comparison of the IntraLase femtosecond laser and mechanical keratomes for laser in situ keratomileusis. J Cataract Refract Surg 2004;30:804-811.

33. Kang PC, McEntire MW, Thompson CJ, et al. Preparation of donor lamellar tissue for deep lamellar endothelial keratoplasty using a microkeratome and artificial anterior chamber system: endothelial cell loss and predictability of lamellar thickness. Ophthalmic Surg Lasers Imaging 2005;36:381-385.

34. Behrens A, Dolorico AM, Kara DT, et al. Precision and accuracy of an artificial anterior chamber system in obtaining corneal lenticules for lamellar keratoplasty. J Cataract Refract Surg 2001;27:16791687.

35. Li L, Behrens A, Sweet PM, et al. Corneal lenticule harvest using a microkeratome and an artificial anterior chamber system at high intrachamber pressure. J Cataract Refract Surg 2002;28:860-865.

36. Price MO, Price FW, Jr. Descemet's stripping with endothelial keratoplasty: comparative outcomes with microkeratome-dissected and manually dissected donor tissue. Ophthalmology 2006;113:1936-1942.

37. Vesaluoma MH, Petroll WM, Perez-Santonja JJ, et al. Laser in situ keratomileusis flap margin: wound healing and complications imaged by in vivo confocal microscopy. Am J Ophthalmol 2000;130:564573.

38. Hu MY, McCulley JP, Cavanagh HD, et al. Comparison of the corneal response to laser in situ keratomileusis with flap creation using the FS15 and FS30 femtosecond lasers: clinical and confocal microscopy findings. J Cataract Refract Surg 2007;33:673-681.

39. Moller-Pedersen T, Cavanagh HD, Petroll WM, et al. Stromal wound healing explains refractive instability and haze development after photorefractive keratectomy: a 1-year confocal microscopic study. Ophthalmology 2000;107:1235-1245. 
40. Moller-Pedersen T, Vogel M, Li HF, et al. Quantification of stromal thinning, epithelial thickness, and corneal haze after photorefractive keratectomy using in vivo confocal microscopy. Ophthalmology 1997;104:360-368.

41. Gooi P, Robinson JW, Brownstein S, et al. Histologic and ultrastructural findings in a case of traumatic graft failure in deep lamellar endothelial keratoplasty: a clinicopathologic case report. Cornea 2007;26:853-857.

42. Kitzmann AS, Bourne WM, Patel SV. Confocal microscopy of a femtosecond laser LASIK flap before separation. Am J Ophthalmol 2007;143:691-693.

43. Hindman HB, McCally RL, Myrowitz E, et al. Evaluation of deep lamellar endothelial keratoplasty surgery using scatterometry and wavefront analyses. Ophthalmology 2007;114:2006-2012.

44. Petroll WM, Goldberg D, Lindsey SS, et al. Confocal assessment of the corneal response to intracorneal lens insertion and laser in situ keratomileusis with flap creation using IntraLase. J Cataract Refract Surg 2006;32:1119-1128.

45. Pels E, Schuchard Y. Organ-culture preservation of human corneas. Doc Ophthalmol 1983;56:147153.

46. Cheng YY, Pels E, Nuijts RM. Femtosecond-laser-assisted Descemet's stripping endothelial keratoplasty. J Cataract Refract Surg 2007;33:152-155. 


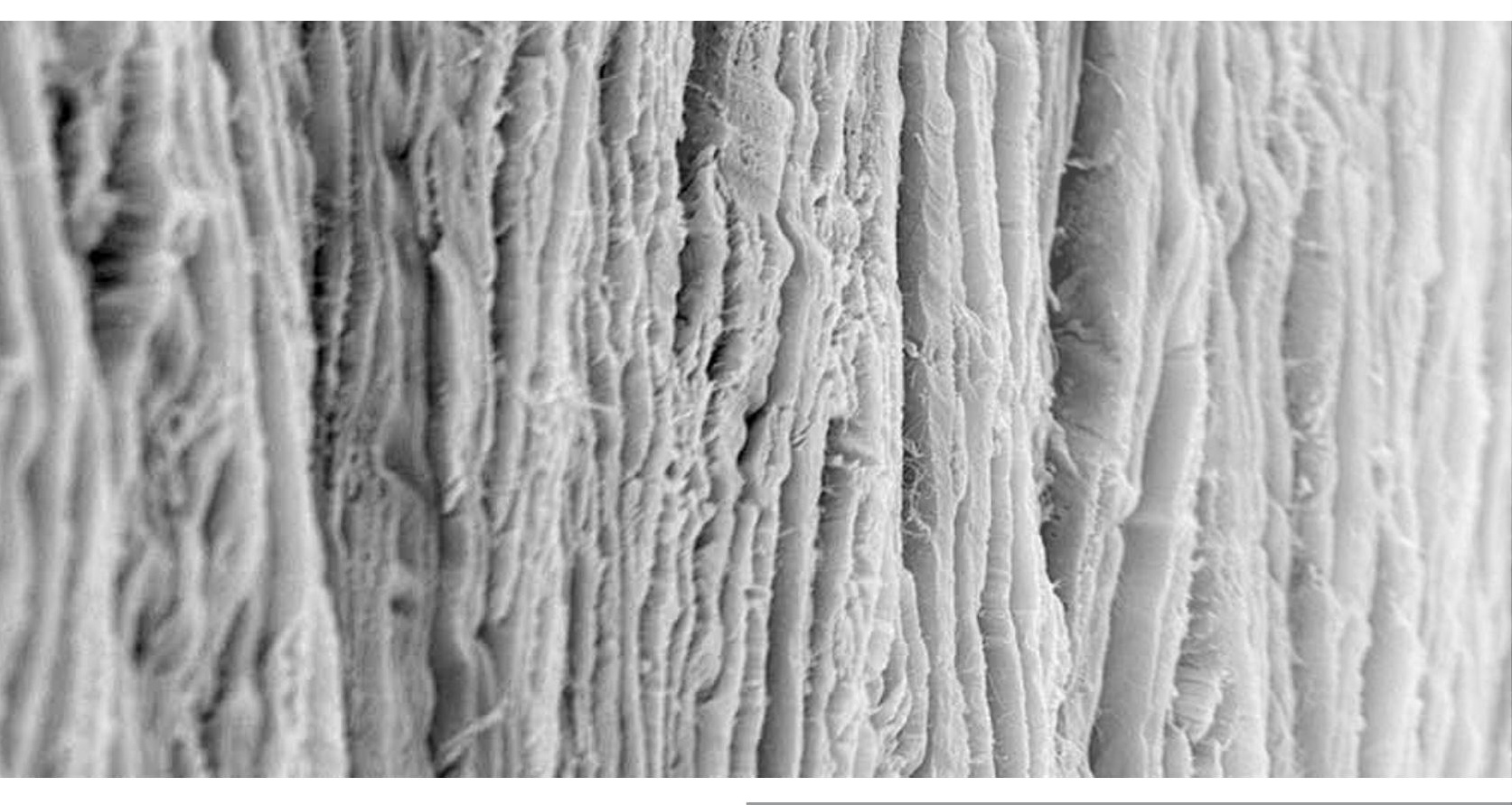




\title{
CHAPTER 3
}

Corneal Endothelial Viability After Femtosecond Laser Preparation of Posterior Lamellar Discs for Descemet-Stripping Endothelial Keratoplasty

\author{
Yanny Y. Y. Cheng \\ Elisabeth Pels \\ Jack P. M. Cleutjens \\ Robert J. van Suylen \\ Fred Hendrikse \\ Rudy M.M.A. Nuijts
}

Cornea 2007 Oct;26(9):1118-1122 


\section{ABSTRACT}

Purpose: To evaluate the feasibility of the femtosecond (FS) laser in preparation of posterior lamellar discs (PLDs) and to study the effect on endothelial cell (EC) viability for Descemet-stripping endothelial keratoplasty.

Methods: Fourteen human donor bulbi unsuitable for transplantation were used. A horizontal lamellar cut was prepared in the donor cornea with an FS laser by using a raster and/or a spiral spot pattern. The control group consisted of the paired cornea of the same donor. EC density was evaluated before and after preservation in organ culture. The PLD was stripped from the anterior part by using either a forceps or a blunt dissection technique. The damage to the endothelium was evaluated.

Results: EC loss after organ storage was not statistically significant between the FS cornea group and the control group in the $15-(7.7 \% \pm 6.9 \%$ and $8.9 \% \pm 8.1 \%$, respectively; $P=0.78)$ and $30-\mathrm{kHz}(4.3 \% \pm 4.0 \%$ and $3.7 \% \pm 3.6 \%$, respectively; $P=0.75)$ group. There was no significant effect of laser frequency ( 15 vs. $30 \mathrm{kHz})$ on EC loss $(7.7 \%$ vs. $4.3 \%, P=$ 0.25). Dissection by using a forceps stripping technique resulted in higher $\mathrm{EC}$ loss than that with a blunt dissection technique $(13.0 \%$ vs. $6.5 \%, P=0.03)$.

Conclusions: EC loss after FS laser lamellar cutting is not dependent on the frequency (ie, energy level) of the laser. A blunt dissection technique of PLDs resulted in acceptable EC loss and supports the clinical use of the FS laser for the preparation of PLDs. 


\section{INTRODUCTION}

Posterior lamellar keratoplasty (PLK), also named deep lamellar endothelial keratoplasty (DLEK), has become an increasingly popular corneal transplantation technique in recent years. PLK and DLEK are manual techniques and have been introduced for the selective transplantation of only the diseased posterior corneal layer in pseudophakic or aphakic bullous keratopathy and in Fuchs endothelial dystrophy. ${ }^{1-3}$ Unfortunately, because of the technical challenges, PLK and DLEK have not been widely adapted yet among corneal transplant surgeons. The preoperative preparation of the posterior lamellar discs (PLDs) from a donor eye, consisting of posterior stroma with the Descemet membrane and endothelium, is time consuming, technically difficult, and not standardized. Therefore, penetrating keratoplasty $(\mathrm{PK})$ is still the most frequently performed procedure for endothelial disease worldwide.

To eliminate the difficult dissection of the pocket in the recipient cornea and to create a smoother surface of the recipient cornea, stripping of the Descemet membrane was introduced. This so-called Descemet-stripping endothelial keratoplasty (DSEK) technique leaves the structure of the posterior cornea intact. ${ }^{4}$ The most recent innovation in DSEK is the use of an automated microkeratome to facilitate an easier preparation of the donor PLDs. ${ }^{5-7}$ Alternatively, the PLD can be prepared by using a femtosecond (FS) laser on a whole donor eye or on an artificial anterior chamber. In The Netherlands and most other European countries, organ culture is the preferred method of storage for corneal donor buttons. ${ }^{8}$ The FS laser-prepared corneoscleral buttons may be stored in organ culture for several weeks before transplantation. A good quality of the donor corneal endothelium after FS laser dissection is an important prerequisite for the success of selective endothelial transplantation techniques.

We performed a study to evaluate the feasibility of the FS laser in preparation of PLDs. The effects of variations in laser frequency and the effect of organ culture on the endothelial cell (EC) viability of FS laser-prepared PLDs were determined.

\section{MATERIALS \& METHODS}

\section{Donor bulbi}

Fourteen human donor bulbi, not suitable for transplantation because of corneal scarring $(\mathrm{N}=13)$ and pterygium $(\mathrm{N}=1)$, were obtained from the Cornea Bank Amsterdam, Netherlands Institute for Neuroscience, Amsterdam, The Netherlands. These corneas were unsuitable for transplantation but had EC counts $>2200$ cells $/ \mathrm{mm}^{2}$. Consent had been obtained from the donors for research use. Donor age averaged $67.3 \pm 7.3$ years; 11 donors were men and 3 were women. The bulbi were stored at $4^{\circ} \mathrm{C}$ in a moist chamber 
and subsequently transported to the University Hospital Maastricht, Maastricht, The Netherlands.

\section{Fs laser surgical procedure}

Before the FS laser procedure, slit-lamp microscopy and Orbscan topography (Orbtek Orbscan II, version 3.10.31; Bausch \& Lomb, Munich, Germany) were used. An eye holder with a suction ring (D.O.R.C. International, Zuidland, The Netherlands) was used to immobilize the whole donor eye. The intraocular pressure was checked with a handheld Barraquer tonometer (Bausch and Lomb) and maintained between a range of 40 and $60 \mathrm{~mm} \mathrm{Hg}$.

We used an FS laser (Intralase Corp., Irvine, CA) to prepare a deep horizontal lamellar cut in the deepithelialized cornea. The cornea was flattened with an applanating interface lens. The horizontal lamellar cut was set at a corneal depth of $400 \mu \mathrm{m}$ with a diameter of $9.5 \mathrm{~mm}$ (Fig. 1). In the first 8 donor bulbi, the horizontal lamellar cuts were obtained with a firing rate of $15 \mathrm{kHz}$ and with an energy level of $2.6 \mu \mathrm{J}$. In the following 6 donor bulbi, the firing rate was $30 \mathrm{kHz}$, and the mean energy level was $1.07 \mu \mathrm{J}$ (range, $0.70-1.40 \mu \mathrm{J})$.

We started with a raster or a combination of a raster and spiral pattern by using a $15-\mathrm{kHz}$ laser. After the $30-\mathrm{kHz}$ treatment mode laser became available, a raster pattern was used. The study group was divided into 2 separate frequency groups: $15(\mathrm{~N}=8)$ and $30 \mathrm{kHz}(\mathrm{N}=6)$. In the $15-\mathrm{kHz}$ group, the donor age was $70.6 \pm 5.2$ years versus $62.8 \pm 7.6$

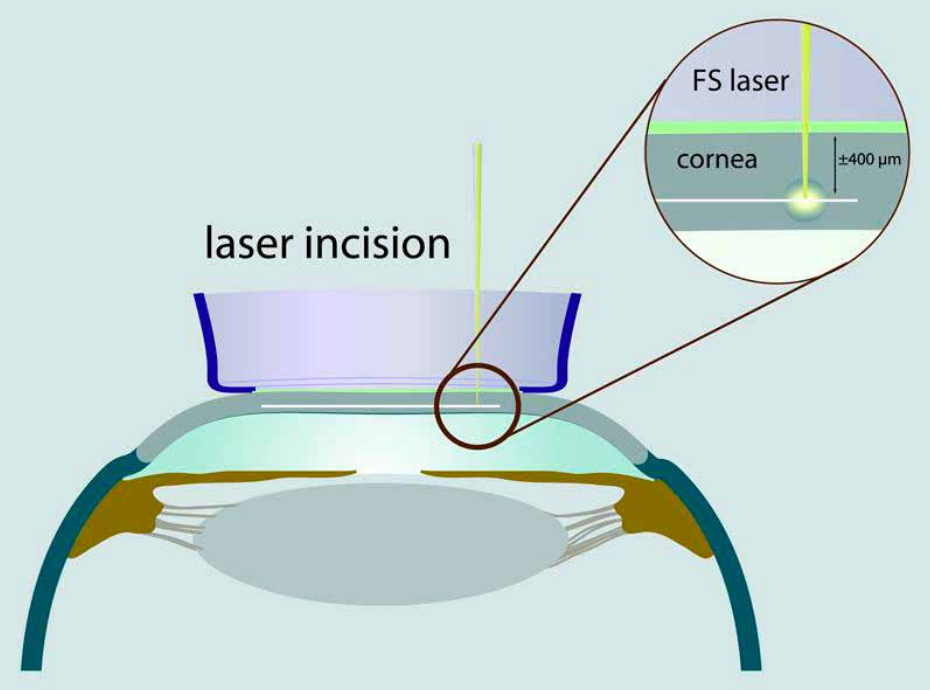

Figure 1. Schematic overview of the horizontal lamellar cut using the FS laser. The cornea was flattened with an applanating interface lens, and a horizontal lamellar cut was set at a depth of $400 \mu \mathrm{m}$. 
years in the $30-\mathrm{kHz}$ group $(P=0.03)$. There was no significant difference in postmortem time between the $15-\mathrm{kHz}$ group and the $30-\mathrm{kHz}$ group $(2.8 \pm 0.2$ and $2.7 \pm 0.6$ days, respectively; $P=0.602$ ).

\section{Donor cornea preparation and ec density}

After the FS laser surgical procedure, the whole donor eye was decontaminated, was stored at $4^{\circ} \mathrm{C}$ in a moist chamber, and subsequently was transported to the Cornea Bank Amsterdam, where a corneoscleral button was prepared. In the Cornea Bank Amsterdam, the EC density was evaluated before and after $7.3 \pm 5.3$ days of storage in organ culture (before dissection of the PLD from the anterior part) by light microscopy after staining with $0.3 \%$ trypan blue in $0.9 \% \mathrm{NaCl}$ (Pharmacy Academic Medical Center, Amsterdam, The Netherlands) for 1 minute. The intercellular spaces were visualized by provoked swelling with $1.8 \%$ sucrose. ${ }^{9}$ Fixed frame counting technique was used to calculate cell density according to the Gundersen method..$^{10}$ The paired cornea of the same donor, suitable for transplantation and untreated, was used as a control in this in vitro study.

After storage in organ culture, the corneoscleral button with a deep horizontal lamellar cut was transported to our clinic. The cornea was punched with an 8.0-mm trephine (Medical Workshop, Groningen, The Netherlands), after which the PLD was removed from the corneal button by using 2 different techniques. The PLD was either stripped from the anterior part of the corneal button by using a forceps (forceps stripping group), or injection of sterile balanced salt solution was used to create an interlamellar space whereupon blunt dissection was performed (blunt dissection group).

The ECs of the PLD were stained with $0.12 \%$ trypan blue in phosphate-buffered saline (Pharmacy Academic Medical Center) for 1 minute, after which the cornea was rinsed with phosphate-buffered saline. The ECs were subsequently stained with $0.2 \%$ alizarin red for 3 minutes and rinsed with phosphate-buffered saline. ${ }^{11}$ EC images of the central and peripheral areas of the PLD were obtained by using light microscopy (Leica DM5000 B; Leica Microsystems, Cambridge, UK). The area of EC damage of 5 subsequent photographs was calculated by using Leica image software (Leica Qwin version 3; Leica Microsystems).

\section{Statistical analysis}

Group differences were assessed by using the Wilcoxon matched-pairs test and the Mann-Whitney $U$ test. Values are reported as mean \pm SD and $95 \%$ confidence intervals (Cls). $P \leq 0.05$ was considered statistically significant. All data analysis was performed by using the SPSS statistical software package (SPSS for Windows version 12.0; SPSS, Chicago, IL). 


\section{RESULTS}

\section{Effect of frequency of the fs laser procedure on ec loss}

There was a slight significant difference between EC density before and after organ culture storage in the FS cornea $15-\mathrm{kHz}$ and FS cornea $30-\mathrm{kHz}$ group $(P=0.03$ and $P=$ 0.05 , respectively; Table 1 ) and in the paired cornea group. After organ culture storage, there was no significant difference between EC loss in the FS cornea group versus the paired cornea group in the $15-(7.7 \% \pm 6.9 \% ; 95 \% \mathrm{Cl}, 1.9-13.5$, and $8.9 \% \pm 8.1 \% ; 95 \% \mathrm{Cl}$, $2.2-15.7$, respectively; $P=0.78)$ and $30-\mathrm{kHz}(4.3 \% \pm 4.0 \% ; 95 \% \mathrm{Cl}, 0.2-8.5$, and $3.7 \% \pm$ $3.6 \%, 95 \% \mathrm{Cl},-0.2$ to 7.5 , respectively; $P=0.75$ ) group. The percentage of EC loss after organ culture storage between the FS cornea $15-\mathrm{kHz}$ (Fig. 2) and the FS cornea $30-\mathrm{kHz}$

TABLE 1. Effect of Laser Frequency on EC Density After Organ Culture

\begin{tabular}{|c|c|c|c|c|c|c|}
\hline $\mathbf{k H z}$ & Cornea & $\begin{array}{c}\text { EC Density } \\
\text { Before Storage } \\
(95 \% \mathrm{Cl})\left(\text { cells } / \mathrm{mm}^{2}\right)\end{array}$ & $\begin{array}{c}\text { EC Density } \\
\text { After Storage } \\
(95 \% \mathrm{Cl})\left(\text { cells } / \mathrm{mm}^{2}\right)\end{array}$ & $P * \dagger$ & $\begin{array}{c}\text { EC Loss } \\
(95 \% \mathrm{CI})(\%) \neq\end{array}$ & $P * \S$ \\
\hline \multirow[t]{2}{*}{15} & FS $(N=8)$ & $\begin{array}{c}2498.0 \pm 282.2 \\
(2262.1-2733.9)\end{array}$ & $\begin{array}{c}2302.0 \pm 274.8 \\
(2072.3-2531.7)\end{array}$ & 0.03 & $\begin{array}{c}7.7 \pm 6.9 \\
(1.9-13.5)\end{array}$ & 0.78 \\
\hline & Paired $(\mathrm{N}=8)$ & $\begin{array}{c}2570.8 \pm 172.7 \\
(2426.3-2715.2)\end{array}$ & $\begin{array}{c}2339.6 \pm 246.8 \\
(2133.3-2546.0)\end{array}$ & 0.04 & $\begin{array}{c}8.9 \pm 8.1 \\
(2.2-15.7)\end{array}$ & \\
\hline \multirow[t]{2}{*}{30} & $\mathrm{FS}(\mathrm{N}=6)$ & $\begin{array}{c}2859.7 \pm 267.0 \\
(2579.4-3139.9)\end{array}$ & $\begin{array}{c}2738.7 \pm 311.6 \\
(2411.7-3065.7)\end{array}$ & 0.05 & $\begin{array}{l}4.3 \pm 4.0 \\
(0.2-8.5)\end{array}$ & 0.75 \\
\hline & Paired $(\mathrm{N}=6)$ & $\begin{array}{c}2919.0 \pm 155.4 \\
(2755.9-3082.1)\end{array}$ & $\begin{array}{c}2811.0 \pm 151.9 \\
(2651.6-2970.4)\end{array}$ & 0.05 & $\begin{array}{c}3.7 \pm 3.6 \\
(-0.2 \text { to } 7.5)\end{array}$ & \\
\hline
\end{tabular}

Data are expressed as the mean \pm SD.

* Wilcoxon paired test.

tDifferences in means between ECD before storage and ECD after storage.

₹ Mean endothelial cell loss after storage in organ culture (\%).

§Difference in endothelial cell loss between the FS cornea and the paired cornea after corneal storage.

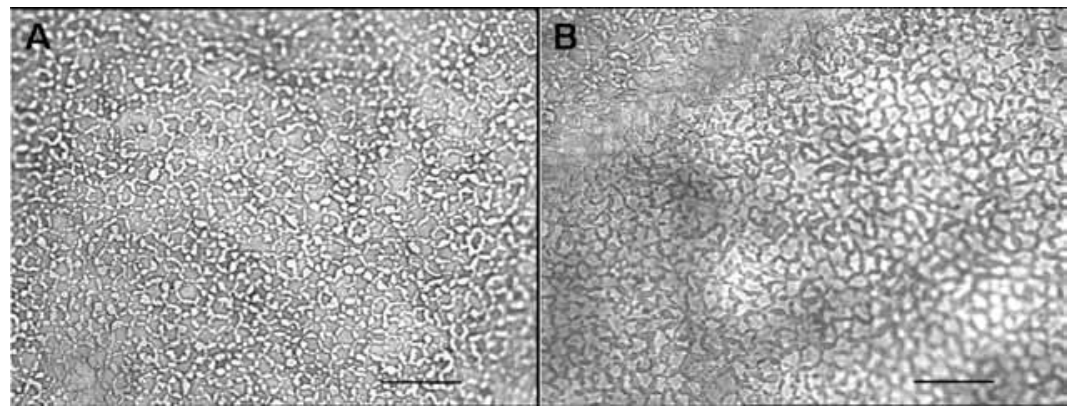

Figure 2. Light microscopy of the ECs of the donor cornea after preparation of a 9.5-mm horizontal lamellar cut at a depth of $400 \mu \mathrm{m}$ with a laser frequency of $15 \mathrm{kHz}$. Endothelium of the donor cornea was stained with trypan blue and swelled with sucrose before $(A)$ and 4 days after (B) organ culture preservation (before separation of the PLD from the anterior part). Average $\mathrm{EC}$ loss after organ culture preservation was $7.7 \% \pm 6.9 \%(\mathrm{~N}=8)$. Scale bar, $100 \mu \mathrm{m}$. 


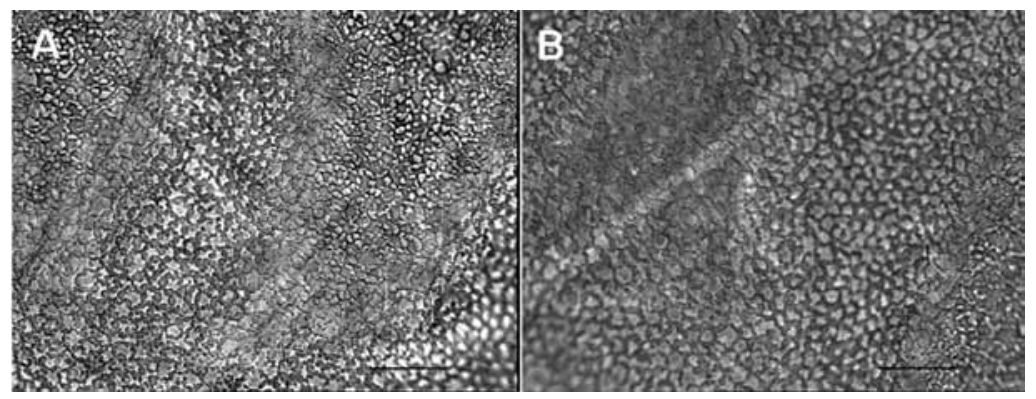

Figure 3. Light microscopy of the ECs of the donor cornea after preparation of a 9.5-mm horizontal lamellar cut at a depth of $400 \mu \mathrm{m}$ with a laser frequency of $30 \mathrm{kHz}$. Endothelium of the donor cornea was stained with trypan blue and swelled with sucrose before (A) and 7 days after (B) organ culture preservation (before separation of the PLD from the anterior part). Average EC loss after organ culture preservation was $4.3 \% \pm 4.0 \%(\mathrm{~N}=6)$. Scale bar, $100 \mu \mathrm{m}$.

(Fig. 3) group was not significant $(7.7 \% \pm 6.9 \%$ and $4.3 \% \pm 4.0 \%$, respectively; $P=0.25)$. The percentage difference of EC loss between the FS cornea $15-\mathrm{kHz}$ and the FS cornea $30-\mathrm{kHz}$ group was $3.4 \%$ (95\% Cl, -3.5 to 10.3 ).

\section{Effect of dissection techniques on ec loss}

In the forceps stripping group, the mean EC loss after organ culture storage was $8.1 \% \pm$ $4.5 \%$ (95\% Cl, 2.5-13.4); after organ culture storage and dissection, EC loss was $13.0 \% \pm$ $5.3 \%(95 \% \mathrm{Cl}, 6.5-19.6 ; P=0.08)$. In the blunt dissection group, the percentage of $\mathrm{EC}$ loss after the organ culture storage was $6.1 \% \pm 6.8 \%(95 \% \mathrm{Cl},-2.4$ to 14.6$)$; after organ culture storage and dissection, the percentage of EC loss was $6.5 \% \pm 3.2 \%(95 \% \mathrm{Cl}, 2.5-10.5$; $P=0.686$ ). The percentages of EC loss after organ culture storage and dissection for the forceps stripping group and blunt dissection group were $13.0 \% \pm 5.3 \%$ and $6.5 \% \pm 3.2 \%$, respectively ( $P=0.03$; Table 2 ; Fig. 4).

TABLE 2. Effect of Dissection on ECD after Organ Culture

\begin{tabular}{|c|c|c|c|c|}
\hline \multirow[b]{2}{*}{ Variable } & \multicolumn{2}{|c|}{ Dissection Groups } & \multirow[b]{2}{*}{$\begin{array}{l}\text { Difference } \dagger \\
(95 \% \mathrm{CI})\end{array}$} & \multirow[b]{2}{*}{$P *$} \\
\hline & $\begin{array}{c}\text { Forceps Stripping } \\
\text { of the } \\
\text { FS cornea } \\
(95 \% \mathrm{Cl})(\mathrm{n}=5)\end{array}$ & $\begin{array}{c}\text { Blunt Dissection } \\
\text { of the } \\
\text { FS cornea } \\
(95 \% \mathrm{Cl})(\mathrm{n}=5)\end{array}$ & & \\
\hline Energy level $(\mu \mathrm{J})$ & 2.6 & $1.38 \pm 0.7$ & - & 0.02 \\
\hline EC loss after organ culture storage (\%) & $\begin{array}{c}8.1 \pm 4.5 \\
(2.5-13.4)\end{array}$ & $\begin{array}{c}6.1 \pm 6.8 \\
(-2.4 \text { to } 14.6)\end{array}$ & $2.0(-6.6$ to 10.4$)$ & 0.46 \\
\hline $\begin{array}{l}\text { EC loss after organ culture storage } \\
\text { and dissection (\%) }\end{array}$ & $\begin{array}{c}13.0 \pm 5.3 \\
(6.5-19.6)\end{array}$ & $\begin{array}{c}6.5 \pm 3.2 \\
(2.5-10.5)\end{array}$ & $6.5(0.1-12.9)$ & 0.03 \\
\hline
\end{tabular}

Data are expressed as the mean \pm SD.

* Mann-Whitney test.

†The percent difference between the forceps stripping group and the blunt dissection group. 


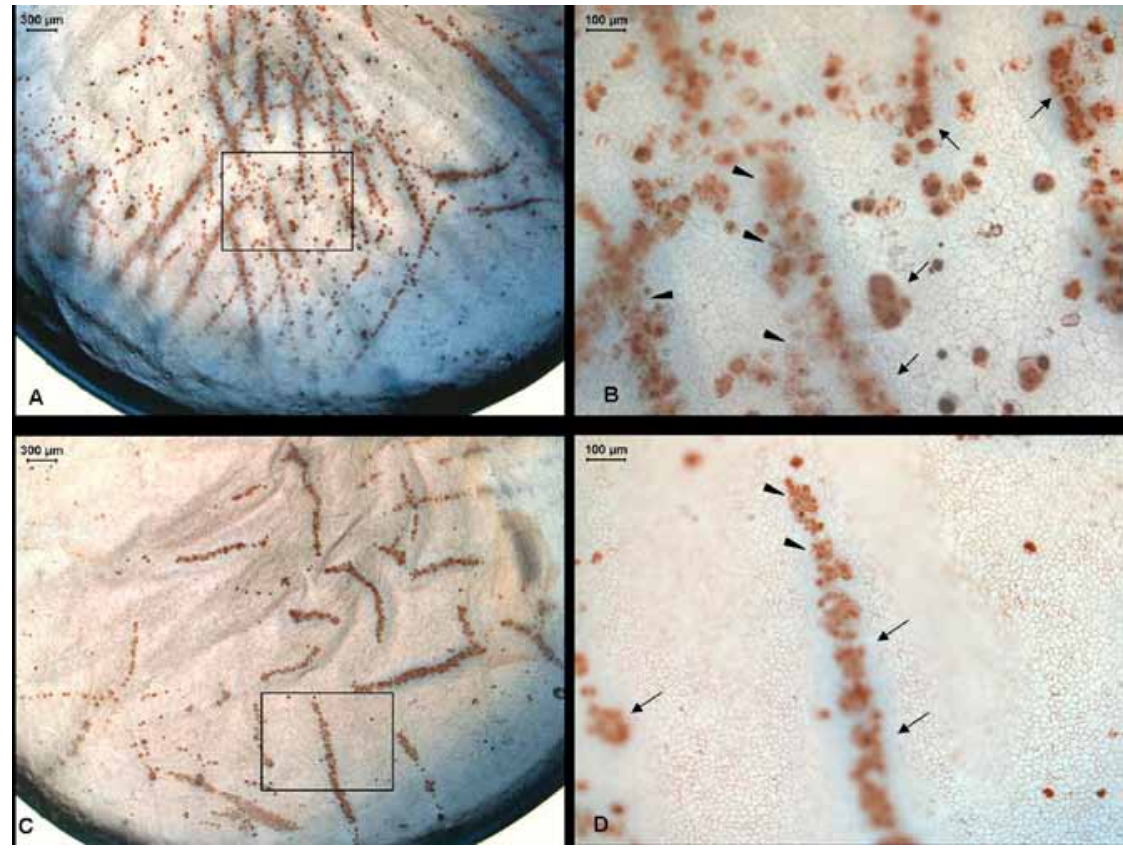

Figure 4. Light microscopy of endothelium of the PLD after combined staining of trypan blue and alizarin red. Trypan blue stained the nuclei of damaged ECs (arrowheads), and alizarin red stained the intercellular junctions and the underlying Descemet membrane (arrows). A and B, Endothelium after organ culture preservation and forceps stripping showed an average EC loss of $13.0 \% \pm 5.3 \%(N=5)$. C and $D$, Endothelium after organ culture and blunt dissection showed an average EC loss of $6.5 \% \pm 3.2 \%(\mathrm{~N}=5)$.

\section{DISCUSSION}

The purpose of this study was to evaluate the feasibility of the FS laser in preparation of PLDs, by studying the effects of organ culture and variations in laser frequency and on EC viability. An important factor in determining the success of corneal transplantations is the quality of the EC layer of the corneal donor button. Clinical studies evaluating endothelial density after PK have shown that there is ongoing EC loss postoperatively, with a postoperative loss of $17 \%$ after 2 months and $71 \%$ after 15 years. ${ }^{12}$ After DLEK that used a large corneoscleral incision $(9.0 \mathrm{~mm})$, there was a cumulative EC loss of $20 \%-27.1 \%$, $27.9 \%$, and $61 \%$ after 1,2 , and 3 years, respectively. ${ }^{13,14}$ DLEK with a small corneoscleral incision $\left(5.0 \mathrm{~mm}\right.$ ) showed EC loss between $15.4 \%$ and $25.0 \%$ after 6 -month follow-up. ${ }^{15,16}$

Our in vitro study showed minimal EC loss in the FS cornea group after organ culture compared with the paired untreated cornea of the same donor. Unfortunately, the direct effect of the FS laser could not be determined, because it was impossible to measure EC density in the swollen corneas right before and immediately after the FS laser procedure. 
The EC loss after lamellar cutting with the $30-\mathrm{kHz}$ laser was $4.3 \%$ versus $7.7 \%$ after cutting with the $15-\mathrm{kHz}$ laser. The $15-\mathrm{kHz}$ laser group has a $3.4 \%$ higher EC loss than the $30-\mathrm{kHz}$ laser group, but the difference between the 2 groups was not significant. Previous studies have evaluated the mean EC loss before and after organ culture storage by using a similar preservation technique as in our study. ${ }^{17-19}$ The organ culture-stored corneas showed a mean EC loss of $5.0 \%-11.0 \%$, which is comparable to the results of our study. We could not exclude that differences existed in viability of the EC between the 2 groups that were independent of the effects of laser treatment or organ culture. The trypan blue light microscopy technique applied in the Cornea Bank Amsterdam allows for EC density measurements only and not for evaluating other parameters of viability that include polymegethism and pleomorphism. In addition, the EC loss in the untreated eyes of the $15-\mathrm{kHz}$ group was $5.2 \%$ higher than in the untreated eyes of the $30-\mathrm{kHz}$ group. Factors that could explain this difference were donor age and postmortem time. Donor age was significantly lower in the 30 - versus $15-\mathrm{kHz}$ group, but there was no difference in postmortem time between groups.

We found a relatively low EC loss after organ culture preservation and separation of the PLD from the anterior part of the cornea by the blunt dissection method (6.5\%) compared with before separation of the PLD (6.1\%). Manual preparation of PLDs induces an average EC loss of $1.0 \%-8.5 \% .{ }^{20,21}$ When we used a microkeratome and an artificial anterior-chamber system for the preparation of PLDs, an EC loss of $6.7 \%$ was induced. ${ }^{22}$ EC loss after FS laser preparation with applanation from the endothelial side by using hydroxypropylmethyl-cellulose as a protective coupling fluid is reported to be $6.0 \%$. In this study, endothelial cell damage caused by separation of the PLD from the anterior cornea was not reported. ${ }^{23} \mathrm{EC}$ loss after preparation of 150- to 200- $\mu \mathrm{m}$-thick PLDs with the FS laser has been shown to be $4 \%$ and did not appear to be related to the level of pulse energy used. ${ }^{24}$ EC losses after preparation of the PLD with an artificial anteriorchamber system and microkeratome or FS laser after 2-day storage in Optisol GS at $4{ }^{\circ} \mathrm{C}$ were $3.4 \%$ and $1.6 \%$, respectively. ${ }^{25}$

One major advantage of the preparation of the donor tissue by using the FS laser is that the procedure can be performed in an automated and standardized fashion, which reduces the level of technical difficulty in relation to the manual dissection of a PLD. The corneoscleral button with a horizontal lamellar cut can be stored in organ culture up to 3 weeks, thereby allowing time for serologic screening of the donor and microbiologic testing and human leukocyte antigen typing of the tissue.

In summary, EC loss after FS laser lamellar cutting is not dependent on the frequency (ie, energy level) of the laser. A blunt dissection technique for preparation of the PLD seems less traumatic to the corneal endothelium than a forceps stripping technique. The dissection of a PLD from the anterior cornea by using a blunt dissection technique does not result in a significant additional cell loss. This finding supports the clinical use 
of the FS laser for the preparation of PLDs for DSEK. A multicenter randomized clinical study is in progress to compare the postoperative EC viability and visual results of FS laser-assisted DSEK with the results of conventional PK. 


\section{REFERENCES}

1. Melles GR, Lander F, Beekhuis WH, et al. Posterior lamellar keratoplasty for a case of pseudophakic bullous keratopathy. Am J Ophthalmol. 1999;127:340-341.

2. Melles GR, Lander F, van Dooren BT, et al. Preliminary clinical results of posterior lamellar keratoplasty through a sclerocorneal pocket incision. Ophthalmology. 2000;107:1850-1856.

3. Terry MA, Ousley PJ. Deep lamellar endothelial keratoplasty in the first United States patients: early clinical results. Cornea. 2001;20:239-243.

4. Melles GR, Wijdh RH, Nieuwendaal CP. A technique to excise the descemet membrane from a recipient cornea (descemetorhexis). Cornea. 2004;23:286-288.

5. Price FW Jr, Price MO. Descemet's stripping with endothelial keratoplasty in 200 eyes: early challenges and techniques to enhance donor adherence. J Cataract Refract Surg. 2006;32:411-418.

6. Price MO, Price FW. Descemet's stripping with endothelial keratoplasty: comparative outcomes with microkeratome-dissected and manually dissected donor tissue. Ophthalmology. 2006;113:1936-1942.

7. Gorovoy MS. Descemet-stripping automated endothelial keratoplasty. Cornea. 2006;25:886-889.

8. Maas-Reijs J, Pels E, Tullo AB. Eye banking in Europe 1991-1995. Acta Ophthalmol Scand. 1997;75:541-543.

9. Pels E, Schuchard Y. Organ culture and endothelial evaluation as a preservation method for human corneas. In: Brightbill FS, ed. Corneal Surgery, Theory, Technique and Tissue. St. Louis, MO: C.V. Mosby; 1986: 93-102.

10. Sperling S, Gundersen HJ. The precision of unbiased estimates of numerical density of endothelial cells in donor cornea. Acta Ophthalmol (Copenh). 1978;56:793-802.

11. Taylor MJ, Hunt CJ. Dual staining of corneal endothelium with trypan blue and alizarin red S: importance of $\mathrm{pH}$ for the dye-lake reaction. Br J Ophthalmol. 1981;65:815-819.

12. Patel SV, Hodge DO, Bourne WM. Corneal endothelium and postoperative outcomes 15 years after penetrating keratoplasty. Am J Ophthalmol. 2005;139:311-319.

13. Ousley PJ, Terry MA. Stability of vision, topography, and endothelial cell density from 1 year to 2 years after deep lamellar endothelial keratoplasty surgery. Ophthalmology. 2005;112:50-57.

14. Van Dooren B, Mulder PG, Nieuwendaal CP, et al. Endothelial cell density after posterior lamellar keratoplasty (Melles techniques): 3 years followup. Am J Ophthalmol. 2004;138:211-217.

15. Fogla $R$, Padmanabhan P. Initial results of small incision deep lamellar endothelial keratoplasty (DLEK). Am J Ophthalmol. 2006;141:346-351.

16. Terry MA, Ousley PJ. Deep lamellar endothelial keratoplasty visual acuity, astigmatism, and endothelial survival in a large prospective series. Ophthalmology. 2005;112:1541-1548.

17. Frueh BE, Bohnke M. Prospective, randomized clinical evaluation of Optisol vs organ culture corneal storage media. Arch Ophthalmol. 2000;118:757-760.

18. Thuret G, Chiquet C, Bernal F, et al. Prospective, randomized clinical and endothelial evaluation of 2 storage times for cornea donor tissue in organ culture at 31 degrees C. Arch Ophthalmol. 2003;121:442-450.

19. Pels $E$, Schuchard $Y$. The effects of high molecular weight dextran on the preservation of human corneas. Cornea. 1984;3:219-227.

20. Ignacio TS, Nguyen TT, Sarayba MA, et al. A technique to harvest Descemet's membrane with viable endothelial cells for selective transplantation. Am J Ophthalmol. 2005;139:325-330.

21. Suwan-apichon $O$, Rizen $M$, Reyes JM, et al. A new donor cornea harvesting technique for posterior lamellar keratoplasty. Br J Ophthalmol. 2005;89:1100-1101. 
22. Kang PC, McEntire MW, Thompson CJ, et al. Preparation of donor lamellar tissue for deep lamellar endothelial keratoplasty using a microkeratome and artificial anterior chamber system: endothelial cell loss and predictability of lamellar thickness. Ophthalmic Surg Lasers Imaging. 2005;36:381-385.

23. Sikder S, Snyder RW. Femtosecond laser preparation of donor tissue from the endothelial side. Cornea. 2006;25:416-422.

24. Sarayba MA, Juhasz T, Chuck RS, et al. Femtosecond laser posterior lamellar keratoplasty: a laboratory model. Cornea. 2005;24:328-333.

25. Suwan-Apichon O, Reyes JM, Griffin NB, et al. Microkeratome versus femtosecond laser predissection of corneal grafts for anterior and posterior lamellar keratoplasty. Cornea. 2006;25:966-968. 



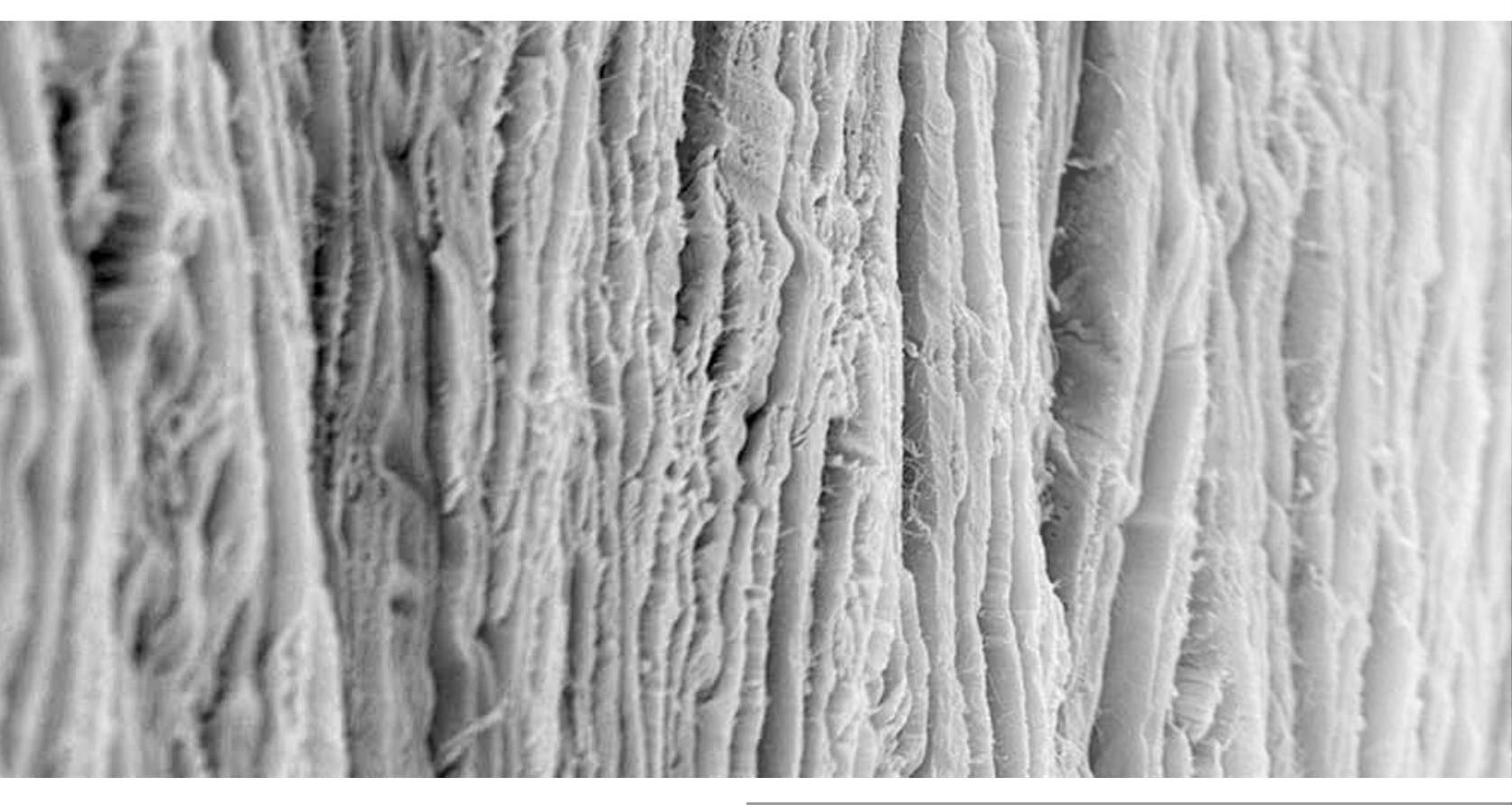




\section{CHAPTER 4}

Femtosecond-Laser-Assisted Descemet's Stripping Endothelial Keratoplasty

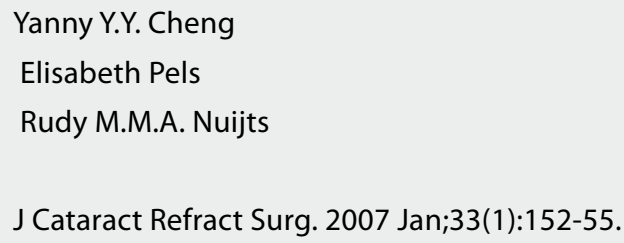




\section{ABSTRACT}

To our knowledge, we describe the first patient with pseudophakic bullous keratoplasty treated with femtosecond-laser-assisted endothelial keratoplasty. A $5.5 \mathrm{~mm}$ corneoscleral tunnel incision was made; after Descemet's membrane was stripped, an $8.0 \mathrm{~mm}$ posterior lamellar corneal disk prepared with a femtosecond laser was inserted into the anterior chamber against the recipient cornea without the use of corneal sutures. Four months postoperatively, the posterior corneal disk was clear and the induced astigmatism was 2.1 diopters, demonstrating a functional corneal endothelial layer. The femtosecond laser offers a new surgical approach for minimally invasive endothelial keratoplasty in corneal endothelial disorders. 


\section{INTRODUCTION}

Full-thickness penetrating keratoplasty (PKP) has been the preferred corneal transplantation technique since the first description of successful corneal transplantation by Zirm in 1906. In PKP, the recipient cornea is trephined in the vertical direction and the donor cornea is sutured into the wound bed using a variety of suture techniques. Although PKP generally results in clear corneal grafts with a graft survival of up to $72 \%$ after 5 years, ${ }^{2,3}$ the procedure is frequently complicated by refractive imperfections and wound-healing problems. ${ }^{4,5}$ Postoperative high, irregular astigmatism and ametropia may result in a significant number of patients who cannot be optically rehabilitated with glasses. Treatment modalities for post-keratoplasty astigmatism and ametropia are rigid gas-permeable contact lens wear, corneal laser refractive surgery, and implantation of toric intraocular lenses. ${ }^{6,7}$ Wound healing after PKP is often unstable and may lead to infection, vascularization, and wound dehiscence, with risks for long-term graft survival.

In recent years, posterior lamellar keratoplasty (PLK), also called deep lamellar endothelial keratoplasty (DLEK), has been described for selective transplantation of the diseased posterior corneal layer in pseudophakic or aphakic bullous keratopathy and Fuchs' endothelial dystrophy. ${ }^{8-10}$ Since surface corneal incisions and sutures are not used in this technique, the corneal shape is preserved, with minimum surgically induced astigmatism and ametropia. However, the perioperative preparation of the posterior corneal disk from a donor eye, consisting of posterior stroma with Descemet's membrane and endothelium, is time-consuming, technically difficult, and not standardized. Because of these technical challenges, PLK is not widely used by corneal transplant surgeons.

We describe a new method of preparing the posterior corneal disk in a standardized and automated fashion using a femtosecond laser, thereby introducing laser technology in corneal endothelial transplant surgery.

\section{Case report}

An 82-year-old man with pseudophakic bullous keratopathy and preexisting maculopathy in the left eye was examined. Investigational review board approval for this study was obtained from the University Hospital Maastricht, and informed consent was obtained from the patient.

The preoperative best corrected visual acuity (BCVA) was counting fingers at $1 \mathrm{~m}$. The topographically simulated keratometry values measured with the Pentacam device (Oculus, Inc.) were 43.6 D @ 9.1 and 37.3 D @ 99.1. The preoperative central corneal thickness was $887 \mu \mathrm{m}$.

A femtosecond laser (IntraLase Corp.) was used to prepare a deep horizontal lamellar interface in the cornea of the donor eye. The postmortem time (ie, period between death of the donor and preparation of the donor posterior corneal disk) was 21 hours, 
and the central corneal thickness of the donor cornea was $856 \mu \mathrm{m}$. The energy level of the laser for preparation of the horizontal lamellar cut at an intended depth of $400 \mu \mathrm{m}$ was $1.4 \mu \mathrm{J}$. The firing rate was $30 \mathrm{kHz}$ and the spot size, $2.4 \mu \mathrm{m}$. A raster spot pattern was used; the diameter of the lamellar cut was $9.5 \mathrm{~mm}$.

The donor eye was placed in an eye holder with a suction ring (DORC International) to immobilize it, and the epithelium was gently removed with a cellulose spear and a surgical Beaver knife. After femtosecond laser treatment, the entire donor eye was cleaned with sterile sodium chloride $0.9 \%$ and thoroughly washed with sterile povidone-iodine solution $0.5 \%$ (for 2 minutes), sterile sodium thiosulfate solution $0.1 \%$ (for 1 minute), and sterile phosphate-buffered saline (for 1 minute) (Cornea Bank of the N.O.R.I.). After decontamination, the donor eye was stored at $4^{\circ} \mathrm{C}$ in a moist chamber. The donor eye was transferred to the Cornea Bank Amsterdam according to the existing protocol for transport of donor tissue in The Netherlands. A corneoscleral rim was prepared and stored in organ culture medium using the Cornea Bank of Amsterdam's certified protocol.

One week after organ culture preservation, an $8.0 \mathrm{~mm}$ corneal button was trephined. The posterior corneal disk was separated from the anterior cornea and inserted through a $5.5 \mathrm{~mm}$ corneoscleral incision into the anterior chamber after a $7.0 \mathrm{~mm}$ circle of Descemet's membrane and endothelium was stripped from the recipient cornea. An air bubble was injected into the anterior chamber and pressed against the posterior corneal disk for 20 minutes, after which most of the air bubble was removed. Two drops of tropicamide minims $0.5 \%$ were instilled to avoid a pupillary block. The goal was to achieve a pupil diameter that was slightly larger than the diameter of the air bubble. No corneal sutures were used for the graft. The corneoscleral incision was closed with a 10-0 nylon suture.

The patient was instructed to remain in a horizontal position during the next 24 hours to maximize the pressure of the air bubble against the posterior corneal disk. (The corneal disk adheres by the endothelial pump mechanism that creates a negative stromal pressure and deturgesces the overlying recipient stroma.) The postoperative treatment consisted of prednisolone acetate $0.5 \% 6$ times daily and chloramphenicol $0.4 \% 3$ times daily.

On the first day, a small cleft of aqueous between the recipient cornea and posterior corneal disk was seen. An air bubble was introduced to fill the anterior chamber and in the recipient cornea, 3, $1.0 \mathrm{~mm}$ full-thickness incisions were made $3.5 \mathrm{~mm}$ from the corneal apex to facilitate drainage of the interlamellar aqueous. One the day after this secondary procedure, the posterior corneal disk was adequately adhered to the recipient cornea.

From 1 week to 2 months postoperatively, the corneal thickness decreased from 794 $\mu \mathrm{m}$ to $593 \mu \mathrm{m}$ (Figure 1) and the residual stromal edema resolved (Figures 2 and 3). Two months after transplantation, the BCVA was 20/60 with a refraction of $+1.5-2.50$ 


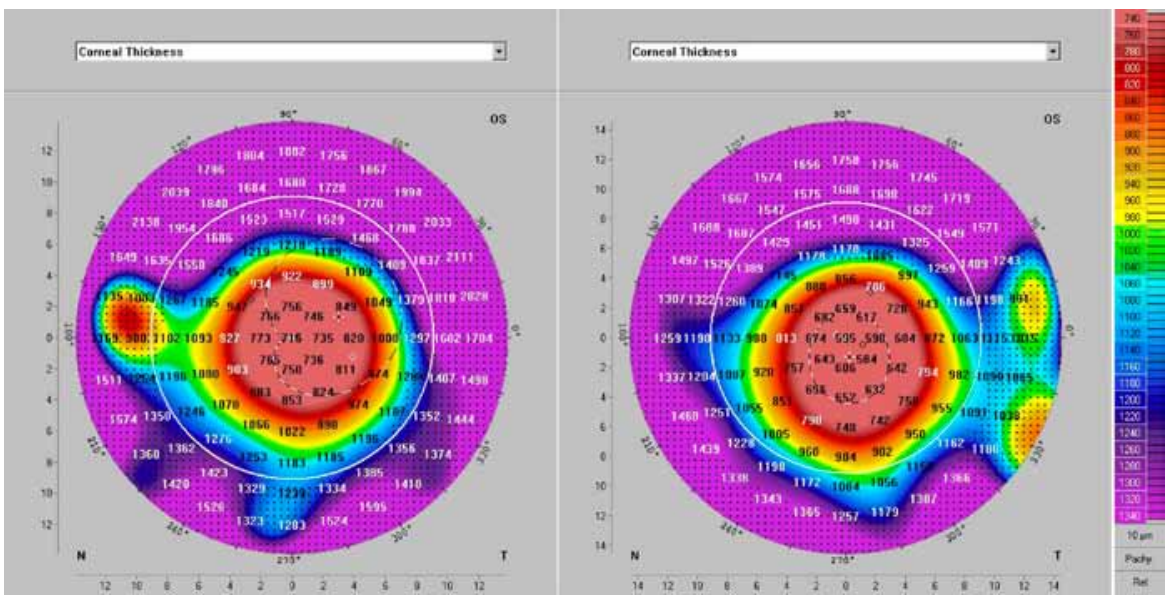

Figure 1. Central corneal thickness after femtosecond DSEK. Central corneal thickness in the left eye measured by Scheimpflug technology (Pentacam) decreased from $794 \mu \mathrm{m} 1$ week postoperatively (left) to $593 \mu \mathrm{m}$ at 2 months (right).

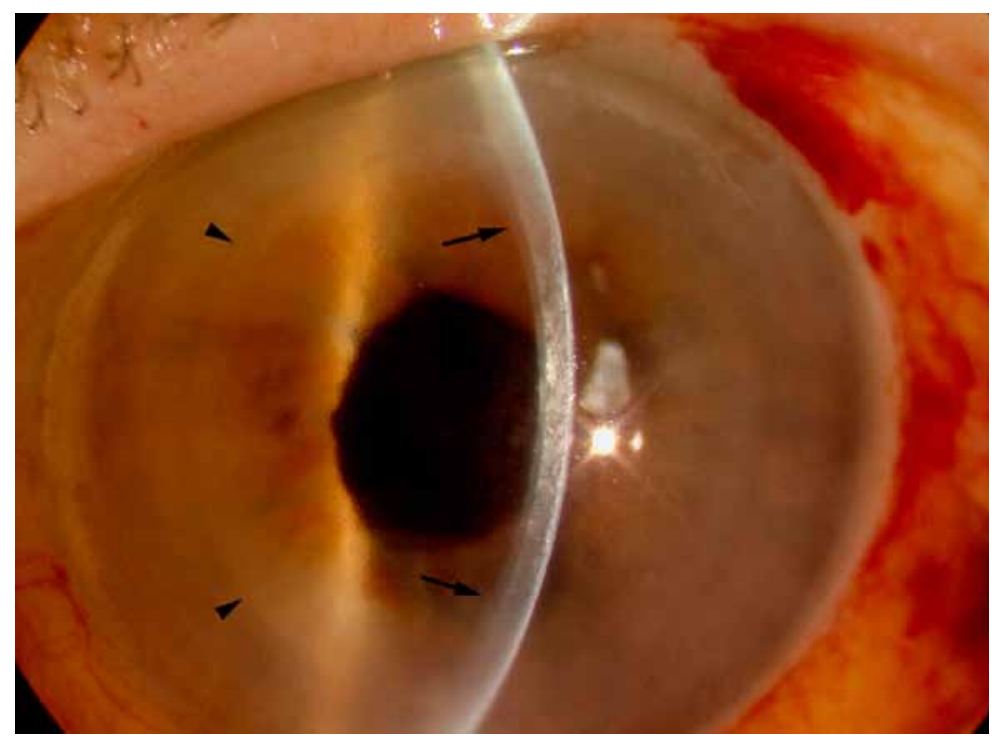

Figure 2. Slitlamp photography of the left eye 1 week after femtosecond DSEK. The margins (arrowhead) of the centrally adhered posterior corneal disk are seen. Some residual stromal edema is visible in the posterior corneal disk (arrow), and central corneal thickness is $794 \mu \mathrm{m}$. 


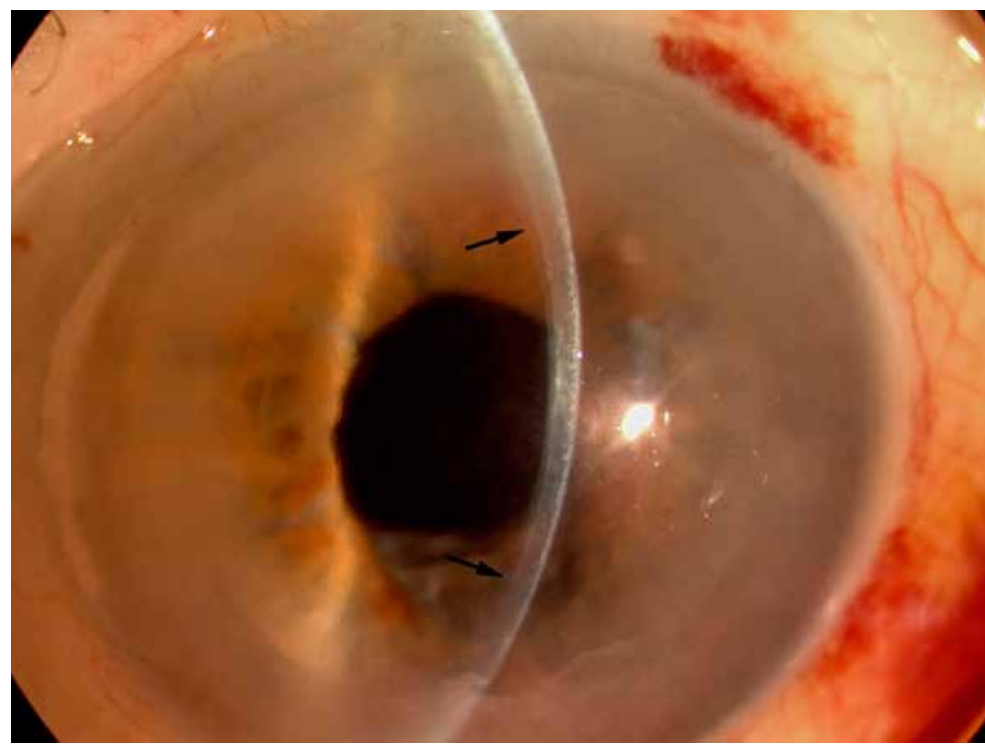

Figure 3. Slitlamp photography of the left eye 2 months after femtosecond DSEK. The stromal edema in the posterior corneal disk has diminished (arrows), and central corneal thickness decreased to $593 \mu \mathrm{m}$.

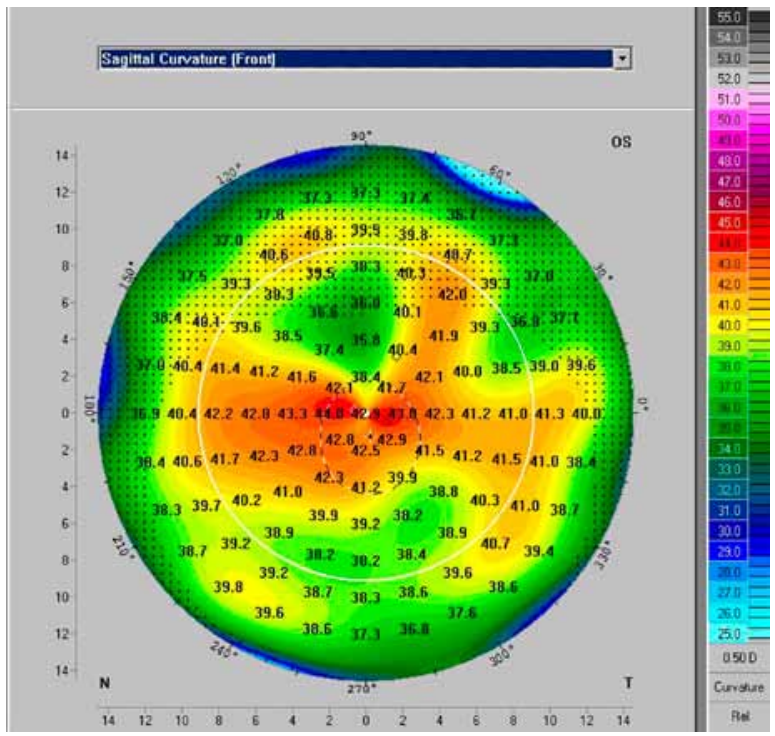

Figure 4. Corneal topography map of the left eye 2 months after femtosecond DSEK. Note the regular topography of the femtosecond DSEK eye with keratometry values of 43.4 D @ 1.3 and 39.4 D @ 91.3. The surgically induced astigmatism by femtosecond DSEK was 2.1 D. 
x 105. The postoperative keratometry values were 43.4 D @ 1.3 and 39.4 D @ 91.3, with surgically induced astigmatism of $2.1 \mathrm{D}$ (Figure 4). Four months postoperatively, the BCVA increased to $20 / 50$ with a refraction of $+1.00-2.25 \times 105$. The keratometry values measured with the EyeMap device (Alcon) were 43.7 D @ 10 and 39.4 D @ 100.

\section{DISCUSSION}

Posterior lamellar keratoplasty was introduced for selective transplantation of the diseased posterior corneal layer in pseudophakic or aphakic bullous keratopathy and Fuchs' endothelial dystrophy. ${ }^{8-10}$ In the original PLK procedure, a large $9.0 \mathrm{~mm}$ corneoscleral incision was made and a deep lamellar pocket in the recipient cornea dissected manually.

To eliminate the difficult dissection of the pocket in the recipient cornea and to create a smoother surface on the recipient cornea, stripping of Descemet's membrane was introduced. ${ }^{11}$ This so-called Descemet's stripping with endothelial keratoplasty (DSEK) technique leaves the structure of the posterior cornea intact and is also less traumatic to the structures in the anterior chamber than DLEK.

The most recent innovation in DSEK is the use of an automated microkeratome to facilitate preparation of the donor posterior corneal disk. After a microkeratome-assisted lamellar cut, the 300 or $350 \mu \mathrm{m}$ anterior part of the donor cornea is removed and the posterior part is punched with an $8.0 \mathrm{~mm}$ trephine.

The advantages of all currently used lamellar endothelial keratoplasty procedures (eg, PLK, DLEK, DSEK) are that no sutures are needed to keep the donor tissue in place, which prevents the occurrence of suture- or incision induced high, irregular astigmatism, the most frequent complication after PKP. ${ }^{9,12-15}$ Visual acuity and corneal topographic data after DLEK remain stable up to 2 years postoperatively. ${ }^{15}$ Endothelial cell loss after these procedures has been shown to be comparable to that after full-thickness PKP. ${ }^{15,16} \mathrm{~A}$ disadvantage of the lamellar techniques compared with full-thickness PKP is that interface opacification at the 2 lamellar surfaces may result in a reduction of the potential BCVA. ${ }^{17}$

In recent years, the femtosecond laser has been used as a microkeratome in laser in situ keratomileusis (LASIK) for cutting a corneal flap at a depth of 100 to $160 \mu \mathrm{m}$. In this refractive surgery technique, excellent visual results without optical interface problems have been noted after a superficial lamellar interface has been created. ${ }^{18,19}$ Consequently, the feasibility of using femtosecond laser technology to prepare a smooth and deep lamellar interface was evaluated in multiple in vitro studies.

To improve and standardize the technique for harvesting the posterior corneal disk, Seitz et al. ${ }^{20}$ demonstrate the feasibility of a femtosecond laser (Femtec, 20/10 Perfect Vision) for nonmechanical endothelial keratoplasty in an in vitro study with human 
donor corneas. Electron microscopy displayed smooth cut surfaces and normal adjacent collagen fibers without evidence of thermal damage. Another study of human cadaver eyes ${ }^{21}$ reports that a femtosecond laser can create deep lamellar dissection smoothness comparable to that of manual dissections in recipient and donor tissues. In a further study of human eyes and corneoscleral buttons, ${ }^{22}$ scanning electron microscopy showed a slightly more stucco-like texture of the interface of deep cuts than in the cuts made by standard anterior surface femtosecond LASIK. Endothelial cell loss after preparation of 150 to $200 \mu \mathrm{m}$ thick posterior stromal disks with the femtosecond laser was about $4 \%$ and did not appear to be caused by laser pulse energy. ${ }^{23}$

A significant advantage of the use of the femtosecond laser in DSEK is that the laser preparation of the donor tissue can be performed in an automated and standardized fashion, which reduces the technical difficulties involved in manual dissection of a posterior corneal disk. Unlike a microkeratome posterior corneal disk, which is prepared at the time of transplantation, the femtosecond-laser-prepared lamellar interface of the disk can be performed up to 3 weeks before the corneal transplantation. Meanwhile, the corneoscleral button with intact corneal architecture can be stored in organ culture, allowing time for serological screening of the donor and microbiological testing and HLA-typing of the tissue. ${ }^{24}$ Preparing the posterior corneal disk in advance also prevents intraoperative situations in which the intended endothelial keratoplasty procedure has to be converted to PKP because of complications during the manual or microkeratome preparation of the donor disk.

The promising future of the application of femtosecond laser technology to corneal endothelial transplant surgery is shown in the present case report in which rapid visual recovery to an expected postoperative visual acuity of 20/50 occurred 4 months after surgery without significant alteration of the shape of the corneal surface. We believe femtosecond DSEK has a potential for facilitating endothelial keratoplasty in amore standardized, automated fashion, with equivalent results in terms of visual rehabilitation, than manual or microkeratome-assisted endothelial keratoplasty. A multicenter randomized clinical study is in progress to compare the postoperative endothelial cell viability and visual results of femtosecond DSEK and conventional PKP. 


\section{REFERENCES}

1. Zirm E. Eine erfolgreiche totale Keratoplastik. Albrecht von Graefes Arch Ophthalmol 1906; 64:580-593

2. Williams KA, Esterman AJ, Bartlett C, et al. How effective is penetrating corneal transplantation? Factors influencing long-term outcome in multivariate analysis. Transplantation 2006; 81:896-901

3. Williams KA, Muehlberg SM, Lewis RF, Coster DJ. How successful is corneal transplantation? A report from the Australian Corneal Graft Register. Eye 1995; 9:219-227

4. Nagra PK, Hammersmith KM, Rapuano $C J$, et al. Wound dehiscence after penetrating keratoplasty. Cornea 2006; 25:132-135

5. Elder MJ, Stack RR. Globe rupture following penetrating keratoplasty; how often, why, and what can we do to prevent it? Cornea 2004; 23:776-780

6. Vajpayee RB, Sharma N, Sinha R, et al. Laser in-situ keratomileusis after penetrating keratoplasty. Surv Ophthalmol 2003; 48:503-514

7. Nuijts RMMA, Abhilakh Missier KA, Nabar VA, Japing WJ. Artisan toric lens implantation for correction of postkeratoplasty astigmatism.Ophthalmology 2004; 111:1086-1094

8. Melles GRJ, Lander F, Beekhuis WH, et al. Posterior lamellar keratoplasty for a case of pseudophakic bullous keratopathy. Am J Ophthalmol 1999; 127:340-341

9. Melles GRJ, Lander F, van Dooren BTH, et al. Preliminary clinical results of posterior lamellar keratoplasty through a sclerocorneal pocket incision. Ophthalmology 2000;107:1850-1856; discussion by HE Kaufman, 1857

10. Terry MA, Ousley PJ. Deep lamellar endothelial keratoplasty in the first United States patients; early clinical results. Cornea 2001; 20:239-243; errata, 353

11. Melles GRJ, Wijdh RHJ, Nieuwendaal CP. A technique to excise the Descemet membrane from a recipient cornea (descemetorhexis). Cornea 2004; 23:286-288

12. Price FW Jr, Price MO. Descemet's stripping with endothelial keratoplasty in 50 eyes: a refractive neutral corneal transplant. J Refract Surg 2005; 21:339-345

13. Terry MA, Ousley PJ. Replacing the endothelium without corneal surface incisions or sutures; the first United States clinical series using the deep lamellar endothelial keratoplasty procedure. Ophthalmology 2003; 110:755-764; discussion JB Rubenstein, 764

14. Terry MA, Ousley PJ. Deep lamellar endothelial keratoplasty; visual acuity, astigmatism, and endothelial survival in a large prospective series. Ophthalmology 2005; 112:1541-1548; discussion by DD Verdier, 1548-1549

15. Ousley PJ, Terry MA. Stability of vision, topography, and endothelial cell density from 1 year to 2 years after deep lamellar endothelial keratoplasty surgery. Ophthalmology 2005; 112:50-57

16. Van Dooren B, Mulder PGH, Nieuwendaal CP, et al. Endothelial cell density after posterior lamellar keratoplasty (Melles techniques): 3 years follow-up. Am J Ophthalmol 2004; 138:211-217

17. Rich LF. Expanding the scope of lamellar keratoplasty. Trans Am Ophthalmol Soc 1999; 97:771-814

18. Tran DB, Sarayba MA, Bor Z, et al. Randomized prospective clinical study comparing induced aberrations with IntraLase and Hansatome flap creation in fellow eyes; potential impact on wavefrontguided laser in situ keratomileusis. J Cataract Refract Surg 2005; 31:97-105

19. Durrie DS, Kezirian GM. Femtosecond laser versus mechanical keratome flaps in wavefront-guided laser in situ keratomileusis: prospective contralateral eye study. J Cataract Refract Surg 2005; 31:120-126 
20. Seitz B, Langenbucher A, Hofmann-Rummelt C, et al. Nonmechanical posterior lamellar keratoplasty using the femtosecond laser (femto-PLAK) for corneal endothelial decompensation. Am J Ophthalmol 2003; 136:769-772

21. Terry MA, Ousley PJ, Will B. A practical femtosecond laser procedure for DLEK endothelial transplantation; cadaver eye histology and topography. Cornea 2005; 24:453-459

22. Soong HK, Mian S, Abbasi O, Juhasz T. Femtosecond laser-assisted posterior lamellar keratoplasty; initial studies of surgical technique in eye bank eyes. Ophthalmology $2005 ; 112: 44-49$

23. Sarayba MA, Juhasz T, Chuck RS, et al. Femtosecond laser posterior lamellar keratoplasty; a laboratory model. Cornea 2005; 24:328-333

24. Pels E, Schuchard Y. Organ-culture preservation of human corneas. Doc Ophthalmol 1983; 56:147-153 



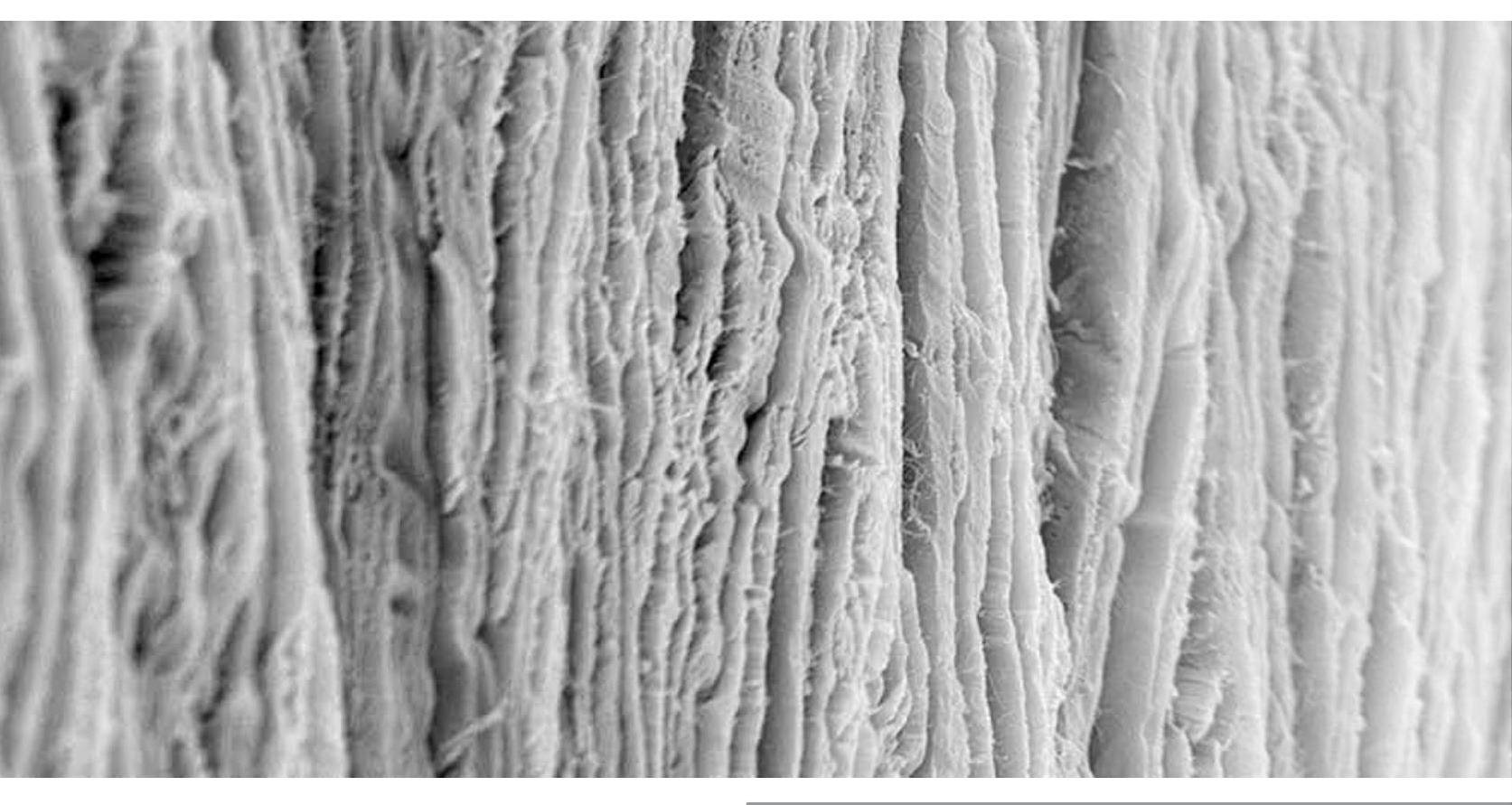




\title{
CHAPTER 5
}

Preliminary Results of

Femtosecond Laser-Assisted

Descemet Stripping Endothelial Keratoplasty

\author{
Yanny Y. Y. Cheng \\ Fred Hendrikse \\ Elisabeth Pels \\ Robert-Jan Wijdh \\ Hugo van Cleynenbreugel \\ Cathariena A. Eggink \\ Gabriel van Rij \\ Wilhelmina J. Rijneveld \\ Rudy M. M. A. Nuijts
}

Arch Ophthalmol. 2008 Oct;126(10):1351-56 


\section{ABSTRACT}

Objective: To evaluate the preliminary visual results of femtosecond laser-assisted Descemet stripping endothelial keratoplasty (FS-DSEK).

Methods: We prospectively analyzed results of 20 consecutive patients with Fuchs endothelial dystrophy or aphakic/pseudophakic bullous keratopathy who underwent FS-DSEK. Best spectacle-corrected visual acuity (BSCVA), refraction, corneal topography, and endothelial cell density were measured preoperatively and 3 and 6 months after FS-DSEK. Corneal thickness was measured using an optical coherence tomography technique.

Results: The average BSCVA of 11 eyes with normal visual potential significantly improved from 20/110 \pm 4 lines to 20/57 \pm 1 line at 6 months $(P<.007)$. At 6 months, the mean (SD) hyperopic shift was 2.24 (2.3) diopters (D). Preoperative and 6 months postoperative refractive astigmatism were $-0.75(0.9) \mathrm{D}$ and $-1.58(1.1) \mathrm{D}(P=.01)$, but the topographic astigmatism did not change postoperatively $(P=.95)$. Mean (SD) endothelial cell density at 6 months was 1368 (425) cells $/ \mathrm{mm}^{2}$. There was a persistent deswelling of the graft up to 3 months postoperatively. Complications included graft dislocations requiring repositioning (20\%), pupillary block glaucoma (5\%), epithelial ingrowth (5\%), and primary graft failure (5\%).

Conclusions: Femtosecond laser-assisted Descemet stripping endothelial keratoplasty was effective in treating endothelial failure with minimal induced refractive astigmatism, limited improvement of BSCVA, and induction of a hyperopic shift. Endothelial cell count and dislocation rate were significant, which may be related to the surgical technique. 


\section{INTRODUCTION}

Several endothelial keratoplasty (EK) procedures, such as posterior lamellar keratoplasty, ${ }^{1}$ posterior lamellar keratoplasty using the femtosecond laser, ${ }^{2}$ deep lamellar endothelial keratoplasty, ${ }^{3}$ Descemet stripping endothelial keratoplasty, ${ }^{4}$ Descemet stripping automated endothelial keratoplasty (DSAEK), ${ }^{5}$ femtosecond laser-assisted Descemet stripping endothelial keratoplasty (FS-DSEK), ${ }^{6}$ and Descemet membrane endothelial keratoplasty, ${ }^{7}$ allow for selective replacement of the diseased endothelial layer, retaining the healthy recipient anterior corneal stroma. Endothelial keratoplasty techniques result in a rapid visual rehabilitation and minimal change in corneal astigmatism. ${ }^{8,9}$ The purpose of this study was to evaluate the clinical outcomes of the first 20 patients after FS-DSEK, where the FS laser is used to prepare the posterior lamellar disc (PLD).

\section{METHODS}

Twenty eyes of 20 consecutive patients with Fuchs endothelial dystrophy $(n=11)$ or aphakic/pseudophakic bullous keratopathy $(n=9)$ were included in a prospective study. Institutional review board approval and informed consent was obtained.

Preoperatively, the medical history was recorded and all patients underwent an ophthalmologic examination. Manifest refraction was obtained by using Snellen acuity charts, and corneal topography (EyeMap EH-290; Alcon, Fort Worth, Texas) was performed. Best spectacle-corrected visual acuity (BSCVA) was determined using the Early Treatment of Diabetic Retinopathy Study letter charts and converted to Snellen equivalents. ${ }^{10,11}$ Endothelial cell density (ECD) of the donor tissue was obtained from the Cornea Bank Amsterdam. Best spectacle-corrected visual acuity, manifest refraction, corneal topography, and ECD (Noncon Robo SP 8000; Konan, Hyogo, Japan) were performed at 3 and 6 months postoperatively.

At 1 week and 1, 3, and 6 months postoperatively, the thickness of the PLD and the thickness of the recipient corneas were measured using an optical coherence tomography technique (Visante; Carl-Zeiss Meditec, Dublin, California). One high resolution corneal quad scan was taken. Four cross-sectional images were taken at $0^{\circ}$ to $180^{\circ}, 45^{\circ}$ to $225^{\circ}, 90^{\circ}$ to $270^{\circ}$, and $135^{\circ}$ to $315^{\circ}$. Measurements of each image were taken at the vertex of the cornea $(0.0 \mathrm{~mm})$ and at $3.5 \mathrm{~mm}$ on each side of the vertex $(-3.5 \mathrm{~mm}$ and $+3.5 \mathrm{~mm}$ ). The 4 central measurements and 8 peripheral measurements were averaged to a single value to determine the thickness of the recipient cornea and the PLD. 


\section{Donor tissue}

The PLD was prepared with the 30-kHz femtosecond laser (AMO, Uppsala, Sweden and Intralase Corp, Irvine, California). The intended depth of the horizontal lamellar cut was $400 \mu \mathrm{m}$, and the diameter was $9.5 \mathrm{~mm}$ using a raster spot pattern with an energy level of $1.4 \mu \mathrm{J} .^{6}$

\section{Surgical technique}

General anesthesia was used in all patients. A 5.0-mm corneoscleral incision and 2 limbal paracenteses were made. The Descemet membrane was scored with a Price-Sinskey hook (Moria,Anthony, France), and a circle of $7.5 \mathrm{~mm}$ of Descemet membrane and endothelium was stripped from the posterior stroma. A $15^{\circ}$ blade was used to make 3 or 4 transcorneal incisions in the midperipheral recipient cornea to drain fluid between the recipient cornea and PLD. ${ }^{4}$

A 8.0-mm donor corneal disc was trephined from the corneoscleral button, and the PLD was removed from the anterior cornea. The endothelial surface was coated with a small layer of viscoelastic material (Healon; AMO). The PLD was gently folded into a taco configuration, and the folded PLD was grasped and inserted using a Goosey forceps (Moria). The corneoscleral incision was closed with two 10-0 nylon sutures. An air bubble was injected to unfold the PLD and press the PLD against the recipient cornea. After 20 minutes, the bubble was partly removed and 2 drops of $0.5 \%$ tropicamide minims were instilled to avoid a papillary block. After pupillary block occurred in 2 patients, a peripheral iridectomy was routinely performed. The patients were instructed to lie supine for the next 24 hours to maximize the pressure of the remaining air bubble against the PLD. The post-operative treatment consisted of $0.5 \%$ prednisolone acetate 6 times daily and $0.4 \%$ chloramphenicol 3 times daily in a tapering dose.

\section{Data analysis}

Comparisons of preoperative measurements and postoperative measurements were performed using a paired t test for normally distributed data and Wilcoxon signed-rank test for nonnormally distributed data. Values are reported as mean (SD). A $P$ value of <.05 was considered statistically significant. Statistical analysis was performed with SPSS (version 12.0; SPSS Inc, Chicago, Illinois).

\section{RESULTS}

Twenty eyes of 20 patients underwent FS-DSEK (Figure 1).The mean (SD) age at surgery was 70.4 (8.4) years with a mean (SD) follow-up of 27.0 (4.4) weeks. Eight patients required FS-DSEK for pseudophakic bullous keratopathy, and 1 patient required FS-DSEK 
for aphakic bullous keratopathy. The remaining 11 patients had Fuchs endothelial dystrophy and cataract. Two patients underwent a combined phacoemulsification and FS-DSEK procedure. The intraocular lens power was chosen for a postoperative spherical equivalent of -0.50 diopter (D).

The preoperative and postoperative details of all patients are shown in Table 1. One patient (patient 1 ) died prior to his 6-month examination. Before surgery and at 6 months, the average BSCVA of the 20 eyes was 20/150 \pm 4 lines and 20/94 \pm 3 lines ( $P=$ $.03)$, respectively. After excluding the eyes with pre-existing retinal problems, amblyopia, and primary graft failure, the average BSCVA of 11 eyes with normal visual potential improved from 20/110 \pm 4 lines preoperatively to 20/57 \pm 1.0 line (range, 20/38-20/100) at 6 months, and 50\% had an improved BSCVA of more than 2 lines at 6 months (Figure 2). The preoperative BSCVA of the patient with a graft failure was 20/87, and at 3 and 6 months, the BSCVA was finger counting.

The mean (SD) preoperative spherical equivalent and refractive astigmatism were $-0.89(2.7) \mathrm{D}$ and $-0.75(0.9) \mathrm{D}$, compared with 1.30 (1.9) $\mathrm{D}(P=.002)$ (Table 2) and -1.58 (1.1) $\mathrm{D}(P=.01) 6$ months postoperatively. The mean (SD) hyperopic shift was 2.23 (2.3) $\mathrm{D}$ at 6 months postoperatively. The mean (SD) topographic cylinder was 2.28 (1.7) D preoperatively and $1.85(1.1) \mathrm{D} 6$ months postoperatively $(P=.95)$.

Two patients had a surgical peripheral iridectomy during FS-DSEK because of a perioperative pupillary block. Postoperatively, 4 of the 20 patients experienced dislocation of the PLD. These patients underwent repositioning of the PLD with an air bubble between 1 day and 1 week postoperatively. One of the 4 patients underwent a second repositioning of the PLD, and in 2 additional patients, interface fluid was drained. One patient experienced pupillary block glaucoma on the first postoperative day, which was relieved by removing a small amount of air from the anterior chamber. At 1 month postoperatively, epithelial ingrowth was seen in 1 patient (patient 16) (Figure 3), with no progression up to 6 months' follow-up. One patient had an iatrogenic graft failure, and
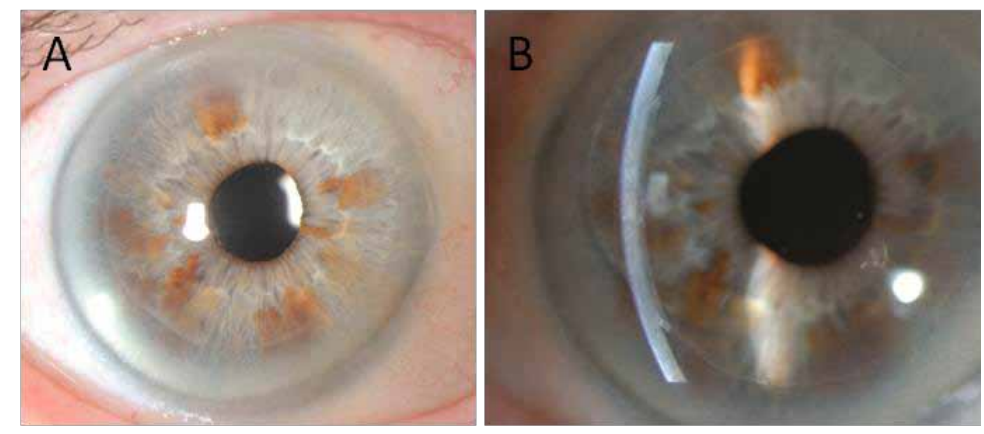

Figure 1. Slitlamp photograph after femtosecond laser-assisted Descemet-stripping endothelial keratoplasty. The cornea is clear, and the graft is well positioned (A and B). 


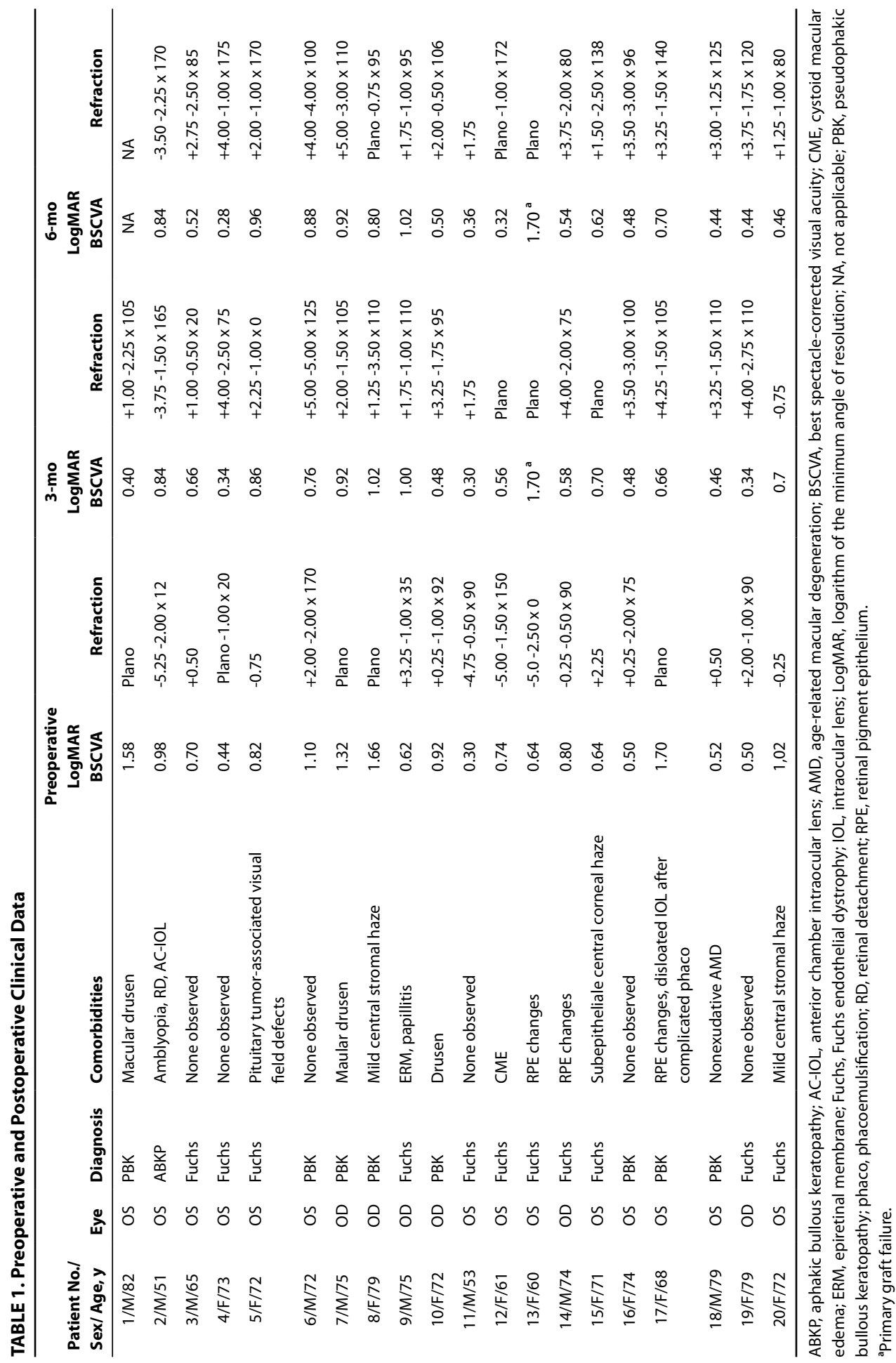




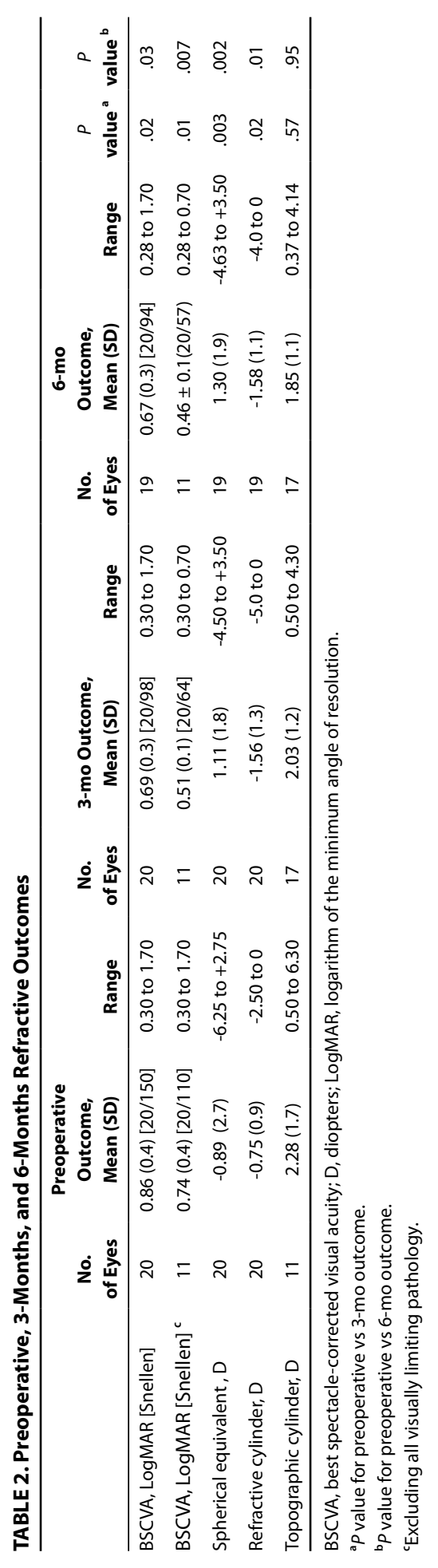




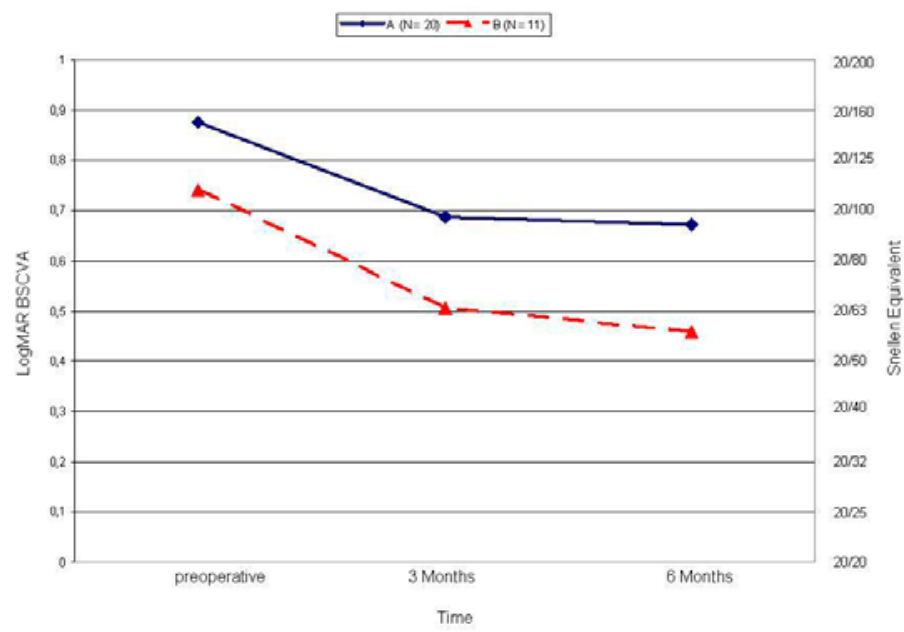

Figure 2. Preoperative and postoperative mean best spectacle-corrected visual acuity (BSCVA), expressed as logarithm of the minimum angle of resolution (LogMAR), and Snellen equivalent for the overall group (line $A$ ) and excluding all visuallimiting pathology (line B).
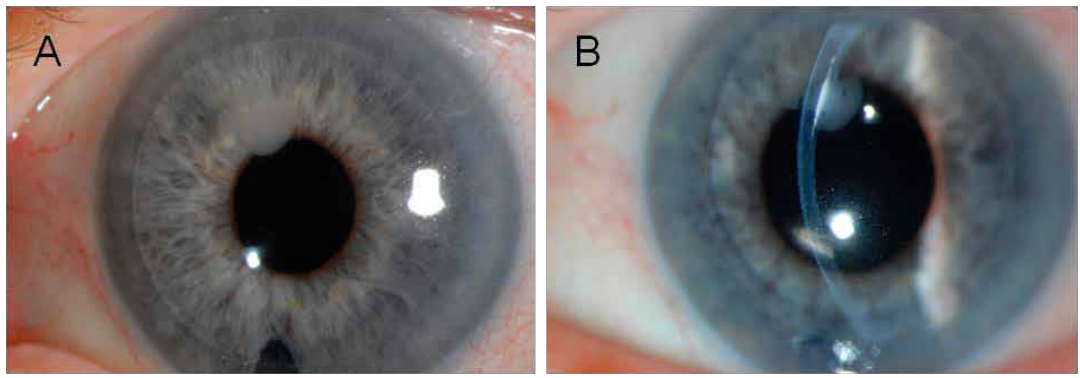

Figure 3. Slitlamp photograph after femtosecond laser-assisted Descemet stripping endothelial keratoplasty. Epithelium in the interface through the transcorneal incision is visible ( $A$ and $B)$.

TABLE 3. Preoperative, 3-Months, and 6-Months ECD

\begin{tabular}{lccc}
\hline & N & $\begin{array}{c}\text { ECD (cells/mm } \mathbf{m}^{\mathbf{2}} \text { ), } \\
\text { Mean (SD) [Range] }\end{array}$ & $\begin{array}{c}\text { \% Cell Loss, } \\
\text { Mean (Range) }\end{array}$ \\
\hline $\begin{array}{l}\text { Eyes without dislocation } \\
\text { Preoperative }\end{array}$ & 16 & $2645(121)[2400-2800]$ & NA \\
$3 \mathrm{mo}$ & 15 & $1325(379)[807-1683]$ & $49(35-70)$ \\
$6 \mathrm{mo}$ & 15 & $1368(425)[761-2108]$ & $48(25-72)$ \\
Eyes with dislocation and repositioning & & & \\
Preoperative & 4 & $2611(176)[2300-2900]$ & NA \\
3 mo & 4 & $753(280)[546-1183]$ & $71(53-80)$ \\
6 mo & 3 & $740(372)[468-1489]$ & $71(43-82)$ \\
\hline
\end{tabular}

$E C D$, endothelial cell density; NA, not applicable. 


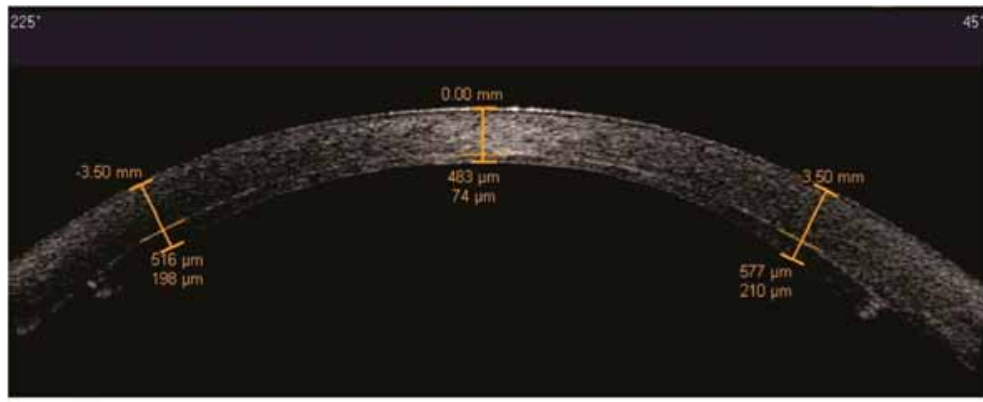

Figure 4. Optical coherence tomography image after femtosecond laser-assisted Descemet stripping endothelial keratoplasty. The corneal thickness at the vertex and $3.5 \mathrm{~mm}$ on each side of the vertex in the $45^{\circ}$ to $225^{\circ}$ meridian.

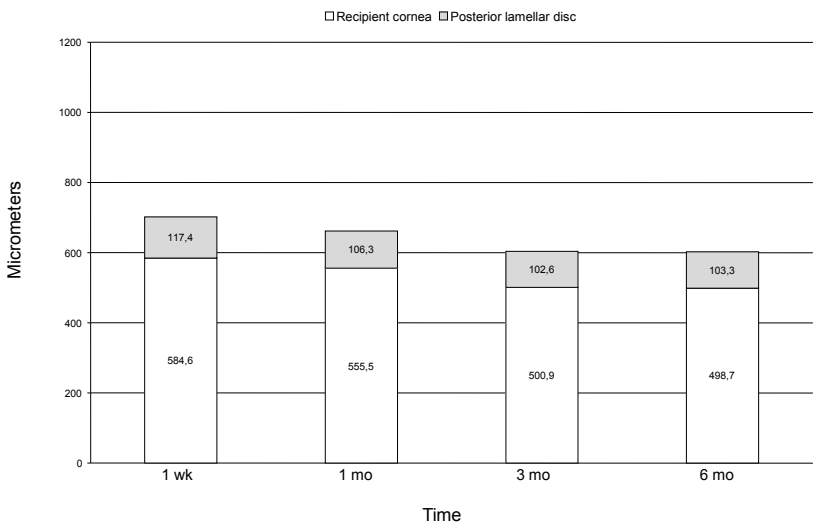

Figure 5. The corneal thickness of the recipient and the posterior lamellar disc at the vertex. At 3 months postoperatively, the thickness of the recipient cornea and posterior lamellar disc had decreased $16.3 \%$ and $16.0 \%$, respectively $(P=.03)$, and remained stable at 6 months.

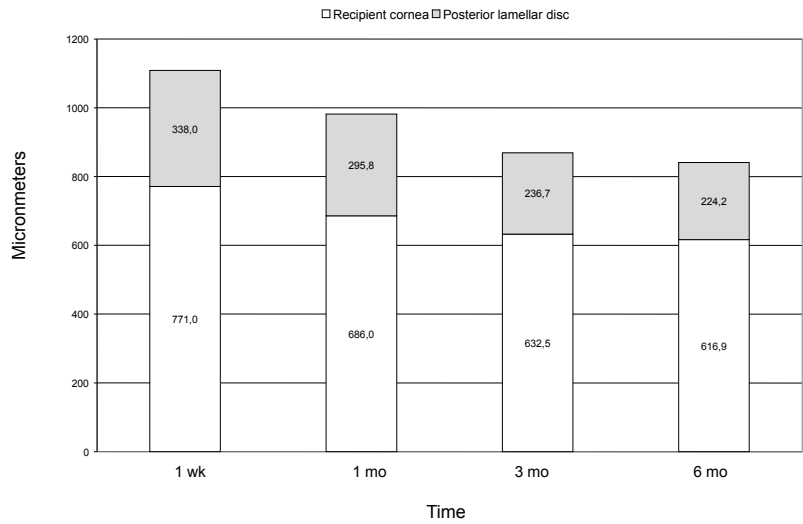

Figure 6. The corneal thickness of the recipient and the posterior lamellar disc at the periphery ( $3.5 \mathrm{~mm}$ of the vertex). At 3 months postoperatively, the thickness of the recipient cornea and posterior lamellar disc had decreased $20.3 \%$ and $29.6 \%$, respectively $(P=.03)$, and remained stable at 6 months. 
after re-FS-DSEK, BSCVA was 20/70. All grafts remained adherent and were clear during the last follow-up visit.

At 6 months postoperatively, the mean (SD) ECD with and without dislocation of the PLD were 740 (372) cells $/ \mathrm{mm}^{2}$ (71\% cell loss) and 1368 (425) cells $/ \mathrm{mm}^{2}$ (48\% cell loss), respectively (Table 3 ).

At 3 months postoperatively, the recipient corneal thickness in the center and periphery had decreased a mean (SD) of $16.3 \%(8.5 \%)(P=.03)$ and $20.3 \%(9.0 \%)(P=.03)$, respectively (Figures 4,5 , and 6 ). The thickness of the PLD in the center and periphery decreased a mean (SD) of $16.0 \%(8.5 \%)(P=.03)$ and $29.6 \%(10.9 \%)(P=.03)$, respectively. The PLD thinned significantly faster at the periphery than at the center $(P=.03)$.

\section{COMMENT}

In a previous in vitro study, we showed that an FS laser is feasible to prepare a PLD for $\mathrm{EK}^{12}$ and in December 2005, we performed the first FS-DSEK. ${ }^{6}$ In the present study, we report the first 20 patients with a follow-up of 6 months after FS-DSEK.

In this first series of FS-DSEK, the average BSCVA improved from 20/110 to 20/57 at 6 months' follow-up, and $50 \%$ of our series with normal vision potential showed an improve-ment of 2 lines or more. After small-incision DSEK, 55\% of the patients had an average BSCVA of 20/40. ${ }^{13,14}$ In a large retrospective series of DSAEK patients with 6 months' follow-up, $69 \%$ of the patients had a BSCVA of $20 / 40$ or better. ${ }^{14}$ In other DSAEK series, an average BSCVA between 20/45 and 20/34 was reported at 3 and 6 months, respectively. ${ }^{5,9,15,16}$ The average BSCVA in our series appears to be lower as compared with recent DSAEK series. A possible explanation for this finding is the quality of the interface at the stromal side of the PLD as prepared by the FS laser. It has been shown that the smoothness of the stromal bed of a PLD prepared with a $15-\mathrm{kHz}$ FS laser is comparable with a manually prepared PLD. ${ }^{17}$ However, Jones et $\mathrm{al}^{18}$ found that a $30-\mathrm{kHz}$ FS laser, using energy levels up to $7.4 \mu \mathrm{J}$, produced a rougher stromal surface than a manual microkeratome. Some authors suggest that the FS laser may cause concentric ridges at the interface disc because of the flat applanation when making the lamellar cut. ${ }^{19}$ Another explanation for the lower than expected BSCVA could be an increase in interface haze after FS-DSEK because of activationof keratocytes, which results in more scatter. $^{20,21}$

Our study showed a mean (SD) hyperopic shift of 2.24 (2.3) D at 6 months postoperatively, which was higher in comparison with other studies. ${ }^{15,16}$ Previous studies of small-incision DSEK or DSAEK have reported a mild hyperopic shift, but other studies did not find a change in spherical equivalent. ${ }^{9,13,14,16,22}$ The shape of the PLD may account for the mild hyperopic shift after small-incision DSEK or DSAEK. The PLDs prepared with 
the microkeratome or the FS laser are thinner in the center and thicker at the edges, as has been shown by our optical coherence tomography results, where the periphery was $53.9 \%$ thicker than the center at 6 months. The intraocular lens selection in our series was targeted to account for emmetropia. The hyperopic shift induced by the PLD should be neutralized by targeting for $-1.00 \mathrm{D}$ to $-1.25 \mathrm{D}$ of myopia when combining cataract procedures with DSEK. ${ }^{23}$

Descemet stripping endothelial keratoplasty or DSAEK results in more predictable postoperative corneal curvature than penetrating keratoplasty., ${ }^{5,16}$ In our study, the change of the topographic astigmatism was not significant, which was comparable with other studies. ${ }^{9,16}$ In contrast to previous DSEK or DSAEK studies, ${ }^{14-16,22}$ which have generally shown no increase in mean refractive astigmatism, our study showed a mild increase in refractive astigmatism, which may be explained by the changes of the posterior corneal curvature. The optical coherence tomography images showed that the thickness of the recipient cornea and the PLD decreased in the first 3 months and remained stable at 6 months. Further, the thickness of the PLD decreased faster in the periphery than in the center, which may influence the posterior corneal curvature and the refraction. ${ }^{23}$ Consequently, centering the PLD on the visual axis may be important to avoid inducing astigmatism.

In the past few years, the surgical techniques for EK have evolved impressively. ${ }^{24,25}$ The major goal of EK is to replace endothelial cells (ECs), as the ECD is an important factor for long-term graft survival. Small incision deep lamellar endothelial keratoplasty showed an EC loss between $15.4 \%$ and $34.0 \%$ after 6 months, ${ }^{8,26,27}$ and the EC loss after smallincision DSEK was between $36 \%$ and $61 \%$ at 12 months. ${ }^{13,28,29}$ The EC loss after DSAEK was between $34 \%$ and $50 \%$ with various follow-up. ${ }^{5,15,29}$ In our series, the EC loss of eyes without dislocation was $48 \%$ at 6 months, which was comparable with the results of small-incision DSEK and DSAEK. Several steps (folding of the PLD, compression of ECs by forceps during insertion, unfolding, long-standing contact with air bubble) during the EK may induce EC loss. ${ }^{30,31}$ In the present multicenter study, FS-DSEK was a new surgical technique for the participating surgeons and the learning curve may have influenced the EC loss.

The most frequently reported complication of small incision DSEK and DSAEK was PLD dislocation. The dislocation rate after small-incision DSEK was between $0.7 \%$ and $50.0 \%$, and after DSAEK, it was between $4.0 \%$ and $34.6 \%$, which was comparable with our study. $4,5,9,14,16,32$

Pupillary block has been reported in between 3.8\% and 9.5\% after DSAEK and occurred peroperatively in 2 of our patients. ${ }^{9,16}$ We suggest that a surgical iridectomy is preferable at the time of DSEK to prevent this complication. One patient in our series had epithelial ingrowth presumably through the transcorneal incision. Epithelium in the interface 9 months after DSEK has been described by Culbertson, ${ }^{33}$ but it was not reported where 
the epithelium originated from. In previous studies, the primary graft failure ranged from $1.0 \%$ to $11.5 \%$, and in our series, 1 graft (5\%) failed because of extensive donor manipulation. 5,9,13,16,32

In summary, FS-DSEK was effective in treating endothelial failure, with minimal change in refractive astigmatism and a mild hyperopic shift in refraction. Although BSCVA improved significantly, we believe that interface issues may result in a lower than expected visual acuity. The EC loss after EK is a concern for long-term graft survival, but this may be related to the steep learning curve of the surgeons. A randomized multicenter study is in progress to compare the visual outcomes and ECD of FS-DSEK with the results of penetrating keratoplasty. 


\section{REFERENCES}

1. Melles GR, Eggink FA, Lander F, et al. A surgical technique for posterior lamellar keratoplasty. Cornea. 1998;17(6):618-626.

2. Seitz B, Langenbucher A, Hofmann-Rummelt C, Schlötzer-Schrehardt U, Nauman GO. Nonmechanical posterior lamellar keratoplasty using the femtosecond Laser (femto-plak) for corneal endothelial decompensation. Am J Ophthalmol.2003;136(4):769-772.

3. Terry MA, Ousley PJ. Deep lamellar endothelial keratoplasty in the first United States patients: early clinical results. Cornea. 2001;20(3):239-243.

4. Price FW Jr, Price MO. Descemet's stripping with endothelial keratoplasty in 200 eyes: early challenges and techniques to enhance donor adherence. J Cataract Refract Surg. 2006;32(3):411-418.

5. Gorovoy MS. Descemet-stripping automated endothelial keratoplasty. Cornea. 2006;25(8):886889.

6. Cheng YY, Pels E, Nuijts RM. Femtosecond-laser-assisted Descemet's stripping endothelial keratoplasty. J Cataract Refract Surg. 2007;33(1):152-155.

7. Melles GR, Ong TS, Ververs B, van der Wees J. Preliminary clinical results of Descemet membrane endothelial keratoplasty. Am J Ophthalmol. 2008;145(2):222-227.

8. Terry MA, Ousley PJ. Deep lamellar endothelial keratoplasty visual acuity, astigmatism, and endothelial survival in a large prospective series. Ophthalmology. 2005;112(9):1541-1548.

9. Koenig SB, Covert DJ. Early results of small-incision Descemet's stripping and automated endothelial keratoplasty. Ophthalmology. 2007;114(2):221-226.

10. Ferris FL III, Kassoff A, Bresnick GH, Bailey I. New visual acuity charts for clinical research. Am J Ophthalmol. 1982;94(1):91-96.

11. Beck RW, Moke PS, Turpin AH, et al. A computerized method of visual acuity testing: adaptation of the early treatment of diabetic retinopathy study testing protocol. Am J Ophthalmol. 2003;135(2):194-205.

12. Cheng YY, Pels E, Cleutjens JP, et al. Corneal endothelial viability after femtosecond laser preparation of posterior lamellar discs for Descemet-stripping endothelial keratoplasty. Cornea. 2007;26(9):1118-1122.

13. Mearza AA, Qureshi MA, Rostron CK. Experience and 12-month results of Descemet stripping endothelial keratoplasty (DSEK) with a small-incision technique. Cornea. 2007;26(3):279-283.

14. Price MO, Price FW Jr. Descemet's stripping with endothelial keratoplasty comparative outcomes with microkeratome-dissected and manually dissected donor tissue. Ophthalmology. 2006;113(11):1936-1942.

15. Koenig SB, Covert DJ, Dupps WJ Jr, Meisler DM. Visual acuity, refractive error, and endothelial cell density six months after Descemet stripping and automated endothelial keratoplasty (DSAEK). Cornea. 2007;26(6):670-674.

16. Covert DJ, Koenig SB. New triple procedure: Descemet's stripping and automated endothelial keratoplasty combined with phacoemulsification and intraocular lens implantation. Ophthalmology. 2007;114(7):1272-1277.

17. Terry MA, Ousley PJ, Will B. A practical femtosecond laser procedure for DLEK endothelial transplantation: cadaver eye histology and topography. Cornea. 2005;24(4):453-459.

18. Jones YJ, Goins KM, Sutphin JE, Mullins R, Skeie JM. Comparison of the femtosecond laser (IntraLase) versus manual microkeratome (Moria ALTK) in dissection of the donor in endothelial keratoplasty: initial study in eye bank eyes. Cornea. 2008;27(1):88-93. 
19. Soong HK, Mian S, Abbasi O, Juhasz T. Femtosecond laser-assisted posterior lamellar keratoplasty: initial studies of surgical technique in eye bank eyes. Ophthalmology. 2005;112(1):44-49.

20. Hindman HB, McCally RL, Myrowitz E, et al. Evaluation of deep lamellar endothelial keratoplasty surgery using scatterometry and wavefront analyses. Ophthalmology. 2007;114(11):2006-2012.

21. Kitzmann AS, Bourne WM, Patel SV. Confocal microscopy of a femtosecond laser LASIK flap before separation. Am J Ophthalmol. 2007;143(4):691-693.

22. PriceFWJr, Price MO. Descemet's stripping with endothelial keratoplasty in 50 eyes: a refractive neutral corneal transplant. J Refract Surg. 2005;21(4):339-345.

23. Holz HA, Meyer JJ, Espandar L, et al. Corneal profile analysis after Descemet stripping endothelial keratoplasty and its relationship to postoperative hyperopic shift. J Cataract Refract Surg. 2008;34(2):211-214.

24. Terry MA. Endothelial keratoplasty: history, current state, and future directions. Cornea. 2006;25(8):873-878.

25. Tan DT, Mehta JS. Future directions in lamellar corneal transplantation. Cornea. 2007;26(9)(suppl 1):S21-S28.

26. Fogla R, Padmanabhan P. Initial results of small incision deep lamellar endothelial keratoplasty (DLEK). Am J Ophthalmol. 2006;141(2):346-351.

27. Yepes N, Segev F, Hyams M, et al. Five-millimeter-incision deep lamellar endothelial keratoplasty: one-year results. Cornea. 2007;26(5):530-533.

28. Price MO, Price FW. Endothelial cell loss after Descemet stripping with endothelial keratoplasty: influencing factors and 2-year trend. Ophthalmology. 2008;115(5):857-865.

29. Terry MA, Chen ES, Shamie N, Hoar KL, Friend DJ. Endothelial cell loss after Descemet's stripping endothelial keratoplasty in a large prospective series. Ophthalmology. 2008;115(3):488-496.e3.

30. Ide T, Yoo SH, Goldman JM, Perez V, O'Brien TP. Descemet-stripping automated endothelial keratoplasty: effect of inserting forceps on DSAEK donor tissue viability by using an in vitro delivery model and vital dye assay. Cornea. 2007; 26(9):1079-1081.

31. Olson RJ. Air and the corneal endothelium: an in vivo specular microscopy study in cats. Arch Ophthalmol. 1980;98(7):1283-1284.

32. Terry MA, Hoar KL, Wall J, Ousley P. Histology of dislocations in endothelial keratoplasty (DSEK and DLEK): a laboratory-based, surgical solution to dislocation in 100 consecutive DSEK cases. Cornea. 2006;25(8):926-932.

33. Culbertson WW. Descemet stripping endothelial keratoplasty. Int Ophthalmol Clin. 2006;46(3):155168. 



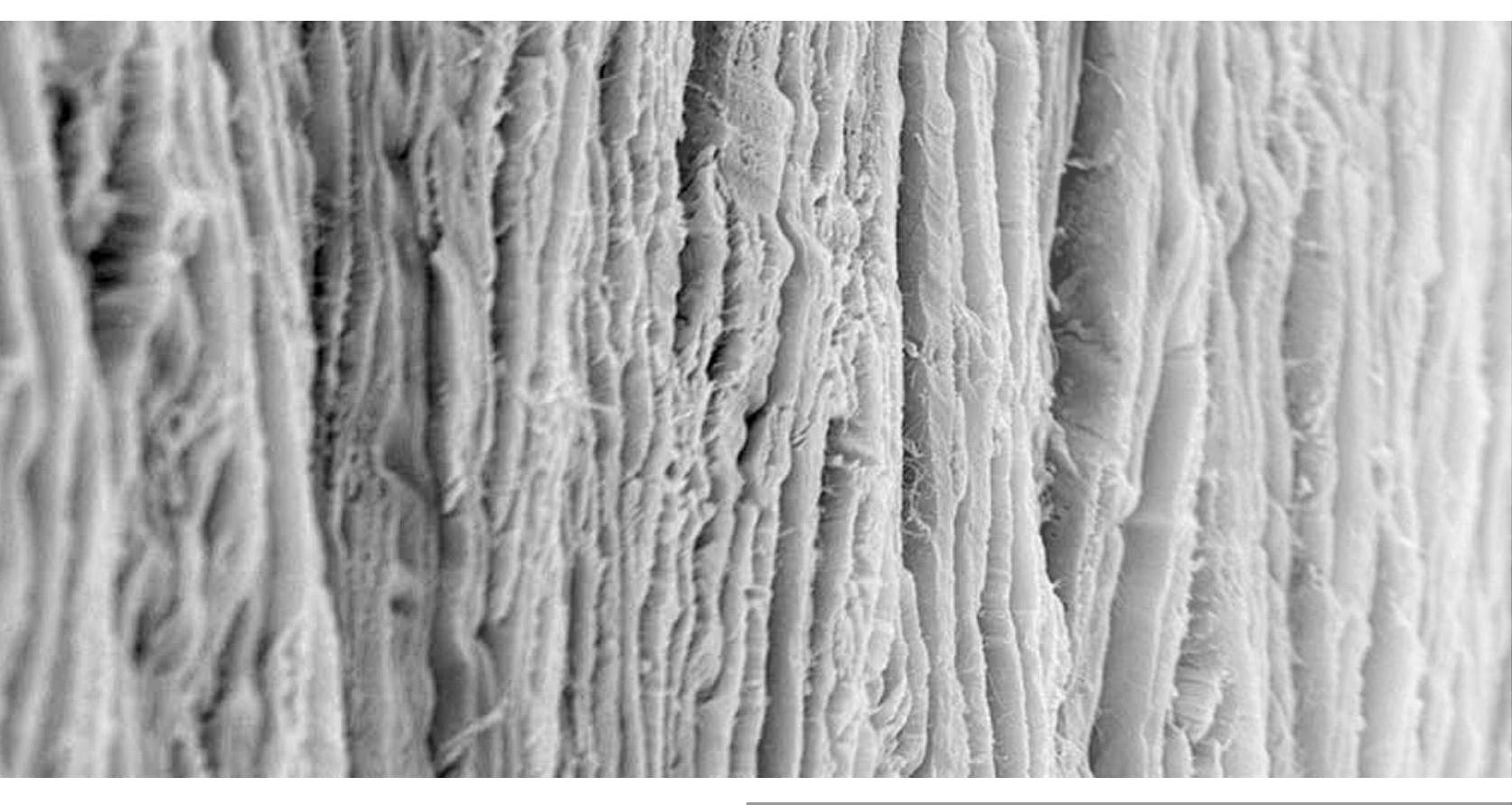




\section{CHAPTER 6}

Efficacy and Safety of

Femtosecond Laser-Assisted

Corneal Endothelial Keratoplasty:

A Randomized Multicenter Clinical

Trial

Yanny Y. Y. Cheng

Jan S. A. G. Schouten

Nayyirih G. Tahzib

Robert-Jan Wijdh

Elisabeth Pels

Hugo van Cleynenbreugel

Catharina A. Eggink,

Wilhelmina J. Rijneveld

Rudy M. M. A. Nuijts

Transplantation. 2009 Dec 15;88(11):1294-302 


\section{ABSTRACT}

Background: To evaluate the efficacy and safety of femtosecond laser-assisted endothelial keratoplasty (FLEK) versus penetrating keratoplasty (PK) in patients with corneal endothelial disease.

Methods: A randomized multicenter clinical trial of 80 eyes of 80 patients with corneal endothelial disease were randomized to FLEK or PK. Clinical outcomes (astigmatism and visual acuity) and incidence of postoperative complications were compared between the two groups.

Results: At 12 months, the percentage of eyes with a refractive astigmatism less than or equal to 3 diopters was higher in the FLEK group in comparison with the PK group (86.2\% vs. $51.3 \%, P=0.004)$. The mean postoperative best corrected visual acuity was $20 / 70 \pm 2$ lines in the FLEK group and 20/44 \pm 2 lines in the PK group $(P<0.001)$, but the gain in the best corrected visual acuity between the two groups was not significantly different. The endothelial cell loss in the FLEK and PK group was $65 \pm 12 \%$ and $23 \pm 15 \%$ $(P<0.001)$. The most common postoperative complication in the FLEK group was graft dislocation (27.8\%). Wound healing related problems occurred in six eyes (15\%) in the PK group and in none of the FLEK eyes.

Conclusions: FLEK effectively reduces postoperative astigmatism and results in an absence of wound healing related problems in patients with endothelial disease. However, visual acuity is lower as compared with conventional PK, and the high level of endothelial cell loss warrants a modification of the insertion technique of the endothelial graft. 


\section{INTRODUCTION}

Penetrating keratoplasty (PK) has been the standard treatment of corneal endothelial disease, and the study has demonstrated that it is a safe and an effective treatment (1). However, dis-advantages of PK are slow visual recovery, high irregular astigmatism, suture-related events, long-term need for topical steroids, postoperative intraocular pressure rise, allograft rejection, and graft failure $(2,3)$.

In recent years, so-called endothelial keratoplasty (EK) techniques are developed for treatment of endothelial disease. These procedures only replace the diseased endothelium, and the healthy anterior stromal tissue can be maintained. Advantages of EK are a rapid visual recovery and fewer wound healing problems (4-6). Techniques for EK have gradually evolved from posterior lamellar keratoplasty (7) and deep lamellar EK (DLEK; 5) to Descemet-stripping EK (DSEK; 6), Descemet-stripping automated EK (DSAEK; 4), femtosecond laser-assisted Descemet-stripping EK (8), and Descemet membrane EK (9). Major technological improvements were the introduction of the descemetorhexis technique (10) and the use of the microkeratome and femtosecond laser for preparing the endothelial graft $(4,8,11-16)$. Reports from the Eye Bank Association of America report a significant increase in demand for donor corneas needed for EK procedures (13.3\% in 2006 vs. $28.2 \%$ in $2007 ; 17)$.

Recently, we showed that a femtosecond laser can be used for the preparation of the endothelial graft $(8,12,18)$. In a country such as The Netherlands, one femtosecond laser based at a cornea bank could be sufficient to produce all tissue for EK procedures. Further-more, the corneoscleral button prepared by the femtosecond laser can be stored in organ culture up to 3 weeks, allowing time for serologic and microbiological screening, tissue typing, and quality control of the donor $(19,20)$.

The Dutch Lamellar Corneal Transplantation Study has been designed to compare visual outcome, amount of endothelial cell loss, complication profile, patient satisfaction, and cost-effectiveness in femtosecond laser-assisted EK (FLEK) versus PK. In this article, we will focus on the safety and efficacy of FLEK, especially its ability to achieve a level of astigmatism at 12 months postoperatively that is compatible with an adequate visual rehabilitation after corneal transplantation.

\section{PATIENTS AND METHODS}

This randomized multicenter trial was conducted at five ophthalmic centers in the Netherlands. The institutional review boards of all participating centers have approved the study. Informed consent was obtained from all patients. The patients were recruited 
between April 2005 and April 2007. The trial was registered at the Netherlands trial register (http://www.trialregister.nl, no. ISRCTN02191620).

Inclusion criteria were patients with Fuchs' endothelial dystrophy, pseudophakic bullous keratopathy, or posterior polymorphous dystrophy, aminimum patient age of 18 years, and best spectacle-corrected visual acuity (BSCVA) lower than 20/50. Exclusion criteria were patients with mental retardation, previous PK, and human leukocyte antigen typed keratoplasty.

The medical history was recorded, and all the patients underwent a comprehensive ophthalmic examination. Preoperative data included patient age, gender, refractive error, intraocular pressure, preoperative lens status, and ocular comorbidities. Endothelial cell density of the donor tissue was obtained from the Cornea Bank Amsterdam, as described previously (19).

\section{Surgical procedures}

In both the FLEK and PK groups, patients with cataract underwent primary cataract extraction with posterior chamber intraocular lens implantation before the corneal transplantation technique or in combination with FLEK or PK. In the FLEK group, patients who were phakic without visually significant cataract also underwent primary cataract extraction and intraocular lens implantation, to avoid iatrogenic crystalline lens touch during the FLEK procedure. All surgical procedures were performed under general anesthesia.

\section{Flek}

In the FLEK group, the donor endothelial graft was prepared with the 30-kHz femtosecond laser (AMO-Intralase, Irvine, CA) as previously described (Fig. 1) (8). In the recipient, a 5.0-mm corneoscleral incision and two limbal paracenteses were made. The Descemet membrane was scored with a Price-Sinskey hook (Moria, Anthony, France), and a circle of 7.5-mm Descemet membrane and endothelium was stripped from the posterior stroma. A 15-degree blade was used to make four transcorneal incisions in the midperipheral recipient cornea to drain fluid between the recipient cornea and endothelial graft (6). An 8.0-mm donor corneal disc was trephined from the corneoscleral button, and the endothelial graft was removed from the anterior cornea. The endothelial surface of the endothelial graft was coated with a small layer of viscoelastic material (Healon, AMO, Uppsala, Sweden), gently folded into a taco-configuration, and inserted using a Goosey forceps (Moria, Anthony, France). The corneoscleral incision was closed with four 10-0 nylon sutures. An air bubble was injected to unfold the endothelial graft and press the endothelial graft against the recipient cornea. After $20 \mathrm{~min}$, the bubble was partly removed, and two drops of tropicamide minims 0.5\% (Chauvin Benelux, Brussel, Belgium) were instilled to avoid a pupillary block. However, after two cases of pupillary 

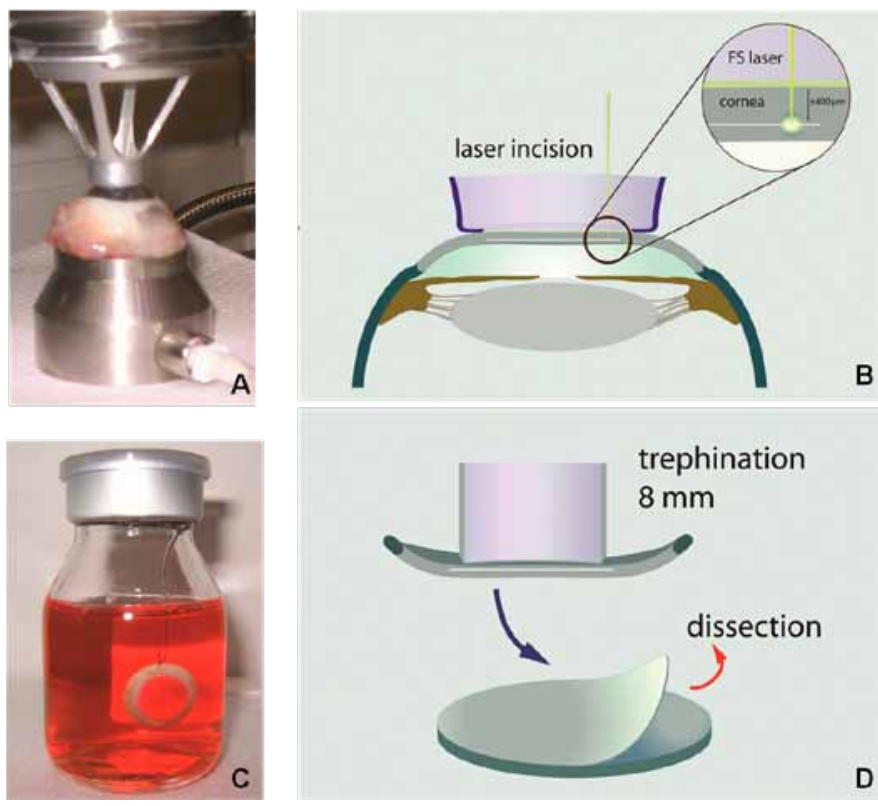

FIGURE 1. (A) The donor cornea is applanated with an applanation cone. (B) A horizontal lamellar cut at a depth of $400 \mu \mathrm{m}$ has been made with the femtosecond laser. (C) After the femtosecond laser procedure, the donor eye has been sent to the Cornea Bank in Amsterdam, and a corneascleral rim has been prepared, and it is stored in organ culture for 2 weeks. (D) During the surgery, the donor scleral rim has been trephined, and the endothelial graft is dissected from the anterior part of the donor cornea. The endothelial graft is used for transplantation.

block had occurred, a peripheral iridectomy was routinely performed. Patients were instructed to lie in supine position during $24 \mathrm{hr}$, to maximize the pressure of the remaining air bubble against the endothelial graft. Postoperatively, all patients received topical dexamethasone $0.1 \%$ drops (Ratiopharm, Zaandam, The Netherlands) 6 times daily and chloramphenicol $0.5 \%$ drops (Ratiopharm, Zaandam, The Netherlands) three times daily in a tapering dose.

\section{Penetrating keratoplasty}

In the PK group, the recipient cornea was trephined using a 7.75- or 8.0-mm HessburgBarron vacuum trephine, and the donor cornea was trephined with an 8.0 - or $8.25-\mathrm{mm}$ disposable trephine. In all cases, a combined suturing technique of a running 11-0 nylon suture with eight interrupted 10-0 nylon sutures was used. Selective suture removal was based on topographic astigmatism pattern. Postoperatively, all patients received topical dexamethasone $0.1 \%$ drops (Ratiopharm, Zaandam, The Netherlands) six times daily and chloramphenicol $0.5 \%$ drops (Ratiopharm, Zaandam, The Netherlands) three times daily in a tapering dose. 


\section{Outcomes measures}

The main primary outcome measure was to assess the percent of eyes with postoperative refractive astigmatism less than or equal to 3.0 diopters (D). Secondary outcome measures included topographical astigmatism (EyeMap EH-290, Alcon), uncorrected visual acuity (UCVA), BSCVA, manifest refraction, endothelial cell density (Noncon Robo, SP 8000, Konan, Hyogo, Japan), and intraoperative and postoperative complications. The UCVA and BSCVA were determined using the Early Treatment of Diabetic Retinopathy Study letter charts and were converted to logarithm of the minimum angle of resolution measurements (21). Vision levels of counting fingers, hand movements, light perception, and no light perception were substituted by logarithm of the minimum angle of resolution values of $1.7,2.0,2.5$, and 3.0, respectively. Preoperatively, and at 3, 6, and 12 months follow-up, all visual parameters were recorded, and the endothelial cell density was measured at 3, 6, and 12 months follow-up.

\section{Sample size}

The sample size was calculated based on the reported amounts of astigmatism less than $3.0 \mathrm{D}$ of $30 \%$ for the PK group and $70 \%$ for the FLEK group (3). Assuming an a of 0.05 and a power of $90 \%, 28$ eyes were needed for analysis in each treatment group.

\section{Randomization}

Consecutive cases from each clinical population were randomly assigned to treatment by FLEK or by PK. The randomization code was generated using a permuted block size of 2. The assigned treatment plans were then sent to the surgeon.

\section{Statistical analysis}

Data were described as mean \pm SD for continuous variables and as individual counts and percentage for categorical variables. The differences between groups were analyzed using a Student's t test for continuous data. The chi-square test or Fisher's exact test were used to compare categorical data. A linear regression model with three dummy variables, which represent the three postoperative moments with the preoperative period as the reference, was used to analyze the change of the three postoperative moments with preoperative measure-ments within a group. We used this model with dummy variables to prevent the problem of multiple testing. If the $P$ value for the three dummies together was less than 0.05 , it was considered as statistically significant. If this $P$ value was lower than 0.05 , the $P$ values of the individual periods were reported and considered statistically significant if $P$ is less than 0.05 . Statistical analysis was performed using SPSS for Windows (version 15.0, SPSS, Inc., Chicago, IL). 


\section{RESULTS}

\section{Participant flow}

The patient flow is displayed in Figure 2. Eighty eyes of 80 patients were recruited, with 40 eyes in each arm. In the FLEK group, four patients did not receive the allocated treatment due to significant preoperative events and were eventually excluded from the study analysis. All patients in the PK group received the allocated treatment.

In the FLEK group, 29 eyes were available for analysis at 12 months follow-up. After surgery, the cornea of three eyes remained edematous and did not clear up, this was defined as primary graft failure. Two eyes underwent PK before the 3 months follow-up, and one eye had repeat FLEK after 6 months follow-up. In one eye with a central corneal fold and another eye with an immunological graft failure, the endothelial grafts were replaced using a DSAEK procedure after 6 months follow-up. Two patients were lost to follow-up due to death. In the PK group, 39 eyes completed the 12 months follow-up. Only one eye was unavailable at the 12 months time point due to health-related issues.

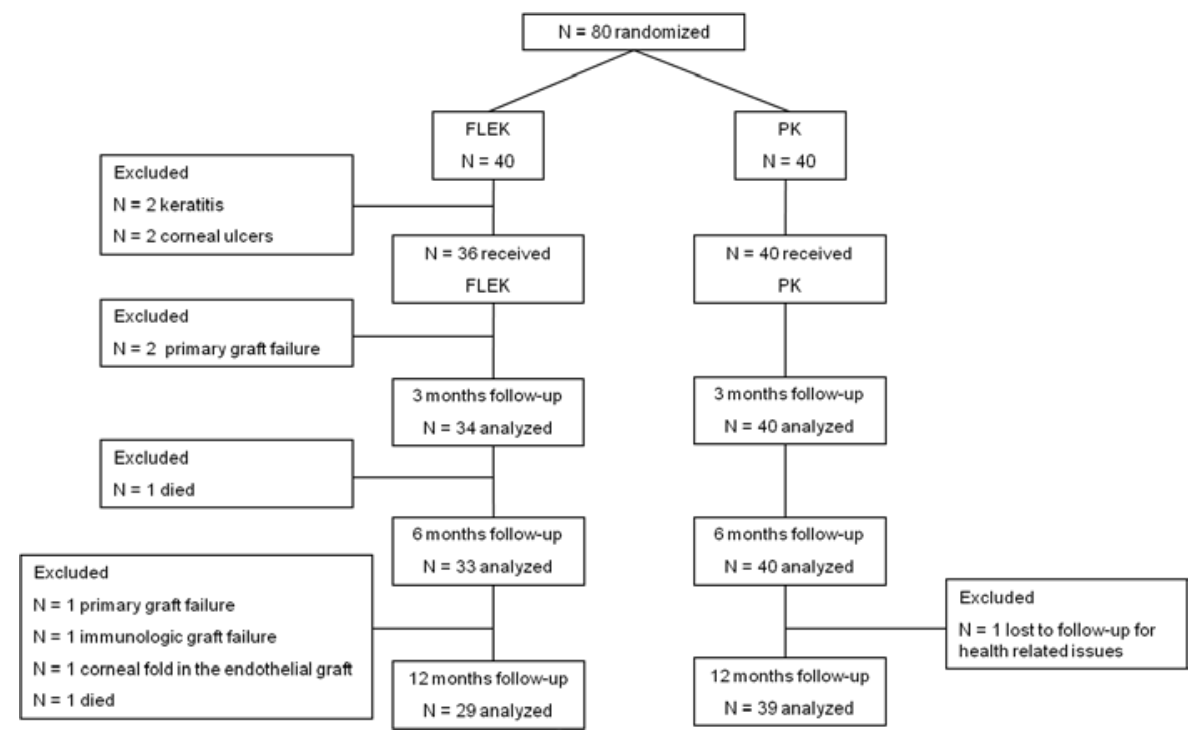

FIGURE 2. Flow diagram of femtosecond laser-assisted endothelial keratoplasty versus penetrating keratoplasty. FLEK, femtosecond laser-assisted endothelial keratoplasty; PK, penetrating keratoplasty.

\section{Patient characteristics}

Patients characteristics of the FLEK and PK groups are listed in Table 1. In the FLEK group, 21 eyes (58.3\%) were diagnosed with Fuchs' endothelial dystrophy and 8 of these 21 
eyes (38.1\%) also had cataract. These eyes underwent primary cataract extraction with intraocular lens implantation ( $n=5$ eyes) followed by the FLEK procedure or a combination of FLEK and cataract extraction with intraocular lens implantation ( $n=3$ eyes). Fifteen eyes (41.7\%) were diagnosed with pseudophakic bullous keratopathy. In the PK group, 20 eyes (50.0\%) were diagnosed with Fuchs' endothelial dystrophy; 12 of these 20 eyes $(60.0 \%)$ also showed cataract. Ten of these 12 eyes (83.3\%) underwent combined procedures of PK and cataract extraction with intraocular lens implantation, and 2 of the 12 eyes underwent primary cataract extraction with intraocular lens implantation before PK.

\section{Refractive outcome and visual outcome}

Preoperatively, there was no significant difference in refractive and topographic astigmatism between the two groups ( $P=0.275$ and $P=0.577$, Table 2$)$. Refractive and topographic astigmatism values remained stable over time in the FLEK group, whereas values were initially higher in the PK group $(P<0.001)$ and showed a tendency to decrease after 3 months time in the PK group. At 12 months postoperatively, the percent of

TABLE 1. Preoperative patient characteristics

\begin{tabular}{|c|c|c|c|}
\hline & FLEK & PK & $P$ \\
\hline Eyes (n) & 36 & 40 & NA \\
\hline Age in years (mean $\pm s d$ ) & $69.0 \pm 8.8$ & $71.4 \pm 11.3$ & 0.308 \\
\hline Women (n, \%) & $21(58.3 \%)$ & $27(67.5 \%)$ & 0.500 \\
\hline Diagnosis & & & 0.725 \\
\hline Fuchs' endothelial dystrophy & $21(58.3 \%)$ & $20(50.0 \%)$ & \\
\hline Pseudophakic bullous keratopathy & $15(41.7 \%)^{a}$ & $19(47.5 \%)^{\mathrm{a}}$ & \\
\hline Posterior polymorphous dystrophy & 0 & $1(2.5 \%)$ & \\
\hline Recipient lens status & & & 0.996 \\
\hline Aphakic & $1(2.8 \%)^{\mathrm{a}}$ & $1(2.5 \%)^{a}$ & \\
\hline Phakic & $21(58.3 \%)$ & $21(52.5 \%)$ & \\
\hline Pseudophakic & $14(38.9 \%)$ & $18(45.0 \%)$ & \\
\hline \multicolumn{4}{|l|}{ Ocular comorbidity } \\
\hline Age-related macular degeneration/ RPE changes & $12(33.3 \%)$ & $5(12.5 \%)$ & 0.030 \\
\hline Cataract & $8(22.2 \%)$ & 12 (30.0\%) & 0.442 \\
\hline Glaucoma & 1 (2.8\%) & $3(7.5 \%)$ & 0.357 \\
\hline
\end{tabular}

a One aphakic eye with iris fixated anterior chamber intra ocular lens. FLEK, femtosecond laser-assisted endothelial keratoplasty; PK, penetrating keratoplasty; SD, standard deviation; NA, not applicable; RPE, retina pigment epithelium. 
eyes with refractive astigmatism less than or equal to $3.0 \mathrm{D}$ was $86.2 \%$ in the FLEK group and $51.3 \%$ in the PK group ( $P=0.004)$.

Preoperatively, there was no significant difference in spherical equivalent between the FLEK and PK groups $(P=0.09)$. At 3, 6, and 12 months postoperatively, FLEK eyes showed a trend toward hyperopia $(P<0.001)$ as compared with PK eyes; the amount of hyperopic shift was $1.77 \pm 2.1 \mathrm{D}, 1.90 \pm 2.2 \mathrm{D}$, and $1.73 \pm 2.0 \mathrm{D}$ at 3,6 , and 12 months follow-up.

Preoperatively and throughout 3,6, and 12 months follow-up, there was no significant difference in UCVA between the FLEK and PK groups (Table 2). Preoperatively, there was no significant difference in BSCVA in the FLEK and PK groups. The mean BSCVA was better after PK compared with FLEK at 3, 6, and 12 months follow-up $(P<0.001)$, but BSCVA gain at 12 months follow-up was not significantly different between FLEK and PK groups $(P=0.103)$.

Compared with preoperatively, BSCVA in the FLEK group improved at $3(P=0.020), 6$ $(P=0.016)$, and at 12 months follow-up $(P<0.001)$. In the PK group, there was significant improvement of BSCVA at all time points $(P<0.001)$.

\section{Endothelial cell density}

The average endothelial cell density of the donor tissue was comparable between the FLEK and PK groups ( $P=0.348$, Table 3). Postoperatively, the average endothelial cell density was significantly lower in the FLEK group compared with the PK group $(P<0.001)$. The average endothelial cell loss in the FLEK versus PK groups at 12 months follow-up was $65 \pm 12 \%$ versus $23 \pm 15 \%(P<0.001)$.

\section{Intraoperative and postoperative complications}

In the FLEK group, a pupillary block occurred in three eyes which needed a surgical peripheral iridectomy (Table 4). Three of 36 eyes (8.3\%) in the FLEK group showed a primary graft failure between 1 week and 1 month. Immunological graft failure occurred in one eye (2.8\%) in the FLEK group, and allograft rejection occurred in one eye (2.5\%) in the PK group. In the FLEK group, epithelial ingrowth was noted in one eye (2.8\%). Ten of 36 eyes $(27.8 \%)$ in the FLEK group showed a dislocation of the endothelial graft, which was repositioned by the injection of an air bubble between day 1 and 1 week postoperatively.

In the PK group, suture-related problems occurred in 4 of the 40 eyes (10.0\%), wound dehiscence in two of the eyes (5.0\%), suture-revision to correct astigmatism in 4 of the 40 eyes (10.0\%). At 12 months follow-up, only one eye in the PK group had all sutures out, whereas the remaining 39 eyes still had most or all their sutures in. No woundrelated problems occurred in the FLEK. 
TABLE 2. Refraction and visual outcomes of FLEK and PK

\begin{tabular}{|c|c|c|c|c|c|}
\hline & \multicolumn{2}{|l|}{ FLEK } & \multicolumn{2}{|l|}{ PK } & \multirow[t]{2}{*}{$P^{a}$} \\
\hline & Mean \pm SD & Range & Mean \pm SD & Range & \\
\hline \multicolumn{6}{|c|}{ Refractive astigmatism (D) } \\
\hline preoperative & $-0.98 \pm 1.0$ & -3.5 to 0 & $-1.27 \pm 1.2$ & -5.50 to 0 & 0.275 \\
\hline $3 \mathrm{mo}$ & $-1.38 \pm 1.2$ & -5.0 to 0 & $-4.17 \pm 3.4^{b}$ & -14.0 to 0 & $<0.001$ \\
\hline $6 \mathrm{mo}$ & $-1.46 \pm 1.3$ & -5.0 to 0 & $-3.21 \pm 1.9^{b}$ & -8.0 to 0 & $<0.001$ \\
\hline \multirow[t]{2}{*}{$12 \mathrm{mo}$} & $-1.22 \pm 1.1$ & -4.0 to 0 & $-2.98 \pm 2.0^{b}$ & -8.75 to 0 & $<0.001$ \\
\hline & $P=0.358^{c}$ & & $P<0.001^{c}$ & & \\
\hline \multicolumn{6}{|c|}{ Topographic astigmatism (D) } \\
\hline preoperative & $1.38 \pm 0.6$ & $0.47-2.90$ & $2.16 \pm 1.4$ & $0.50-6.50$ & 0.577 \\
\hline $3 \mathrm{mo}$ & $1.87 \pm 1.1$ & $0.25-4.30$ & $4.59 \pm 2.9^{b}$ & $1.00-15.70$ & $<0.001$ \\
\hline $6 \mathrm{mo}$ & $1.72 \pm 1.0$ & $0.27-4.14$ & $3.74 \pm 1.7^{b}$ & $0.50-6.83$ & $<0.001$ \\
\hline \multirow[t]{2}{*}{$12 \mathrm{mo}$} & $1.58 \pm 1.2$ & $0.27-6.40$ & $3.67 \pm 1.8^{b}$ & $1.40-7.70$ & $<0.001$ \\
\hline & $P=0.469^{c}$ & & $P<0.001^{c}$ & & \\
\hline \multicolumn{6}{|c|}{ Spherical equivalent (D) } \\
\hline preoperative & $-0.97 \pm 2.4$ & -6.50 to 2.75 & $0.12 \pm 1.9$ & -4.75 to 4.50 & 0.090 \\
\hline $3 \mathrm{mo}$ & $0.84 \pm 1.7^{b}$ & -4.50 to 3.50 & $-0.93 \pm 3.0$ & -6.75 to 6.0 & 0.004 \\
\hline $6 \mathrm{mo}$ & $0.93 \pm 1.7^{b}$ & -4.63 to 3.50 & $-1.47 \pm 3.3$ & -8.25 to 6.75 & $<0.001$ \\
\hline \multirow[t]{2}{*}{$12 \mathrm{mo}$} & $0.78 \pm 1.5^{b}$ & -3.25 to 3.13 & $-1.23 \pm 3.1$ & -7.25 to 7.0 & $<0.001$ \\
\hline & $P<0.001^{c}$ & & $P=0.071^{\mathrm{c}}$ & & \\
\hline \multicolumn{6}{|c|}{ UCVA (logMAR) } \\
\hline preoperative & $1.01 \pm 0.4(20 / 200)$ & $0.22-2.0$ & $0.88 \pm 0.4(20 / 150)$ & $0.40-2.0$ & 0.133 \\
\hline $3 \mathrm{mo}$ & $0.80 \pm 0.2(20 / 125)^{b}$ & $0.40-1.70$ & $0.71 \pm 0.3(20 / 100)^{b}$ & $0.10-1.36$ & 0.144 \\
\hline $6 \mathrm{mo}$ & $0.79 \pm 0.3(20 / 125)^{b}$ & $0.40-1.70$ & $0.79 \pm 0.3(20 / 125)$ & $0.14-1.36$ & 0.959 \\
\hline \multirow[t]{2}{*}{$12 \mathrm{mo}$} & $0.73 \pm 0.3(20 / 102)^{b}$ & $0.34-1.70$ & $0.68 \pm 0.3(20 / 96)^{b}$ & $0.10-1.46$ & 0.539 \\
\hline & $P=0.001^{c}$ & & $P=0.045^{c}$ & & \\
\hline \multicolumn{6}{|c|}{ BSCVA (logMAR) } \\
\hline preoperative & $0.82 \pm 0.4(20 / 132)$ & $0.22-2.0$ & $0.73 \pm 0.4(20 / 105)$ & $0.18-2.0$ & 0.316 \\
\hline $3 \mathrm{mo}$ & $0.65 \pm 0.3(20 / 90)^{b}$ & $0.22-1.70$ & $0.40 \pm 0.2(20 / 50)^{b}$ & -0.04 to 1.12 & $<0.001$ \\
\hline $6 \mathrm{mo}$ & $0.64 \pm 0.3(20 / 87)^{b}$ & $0.26-1.70$ & $0.35 \pm 0.2(20 / 44)^{b}$ & $0-0.86$ & $<0.001$ \\
\hline \multirow[t]{2}{*}{$12 \mathrm{mo}$} & $0.55 \pm 0.2(20 / 70)^{\mathrm{b}}$ & $0.16-1.10$ & $0.35 \pm 0.2(20 / 44)^{b}$ & -0.04 to 0.98 & $<0.001$ \\
\hline & $P=0.004^{c}$ & & $P<0.001^{c}$ & & \\
\hline \multicolumn{6}{|c|}{ BSCVA gain (logMAR) } \\
\hline $3 \mathrm{mo}$ & $0.15 \pm 0.4$ & -1.06 to 1.34 & $0.33 \pm 0.4$ & -0.48 to 1.48 & 0.052 \\
\hline $6 \mathrm{mo}$ & $0.14 \pm 0.4$ & -1.06 to 1.30 & $0.38 \pm 0.4$ & -0.40 to 1.46 & 0.017 \\
\hline $12 \mathrm{mo}$ & $0.24 \pm 0.4$ & -0.34 to 1.50 & $0.38 \pm 0.4$ & -0.24 to 1.30 & 0.103 \\
\hline
\end{tabular}

${ }^{a} P$ value between FLEK and PK.

${ }^{\mathrm{b}} P<0.05$ vs. preoperative in a linear regression model.

' $P$ value of a linear regression model with three postoperative periods at the same time in the model.

FLEK, femtosecond laser-assisted endothelial keratoplasty; PK, penetrating keratoplasty; SD, standard deviation; D, diopter; UCVA, uncorrected visual acuity; logMAR, logarithm of the minimum angle of resolution; BSCVA, best spectaclecorrected visual acuity. 
TABLE 3. Donor and postoperative endothelial cell density of FLEK and PK

\begin{tabular}{|c|c|c|c|c|c|}
\hline & \multicolumn{2}{|c|}{ FLEK } & \multicolumn{2}{|c|}{ PK } & \multirow[t]{2}{*}{$P$ value ${ }^{a}$} \\
\hline & Mean \pm SD & Range & Mean \pm SD & Range & \\
\hline \multicolumn{6}{|c|}{$\begin{array}{l}\text { Endothelial cell densitiy } \\
\text { (cells } / \mathrm{mm}^{2} \text { ) }\end{array}$} \\
\hline Donor & $2678 \pm 159$ & $2300-2900$ & $2653 \pm 143$ & $2400-2900$ & 0.348 \\
\hline $3 \mathrm{mo}$ & $1202 \pm 456^{b}$ & $545-2007$ & $2148 \pm 434^{b}$ & $1065-3083$ & $<0.001$ \\
\hline $6 \mathrm{mo}$ & $1095 \pm 486^{b}$ & $440-2108$ & $2064 \pm 365^{b}$ & $1325-2890$ & $<0.001$ \\
\hline \multirow[t]{2}{*}{$12 \mathrm{mo}$} & $1067 \pm 423^{b}$ & $418-2069$ & $2028 \pm 483^{b}$ & $800-3068$ & $<0.001$ \\
\hline & $P<0.001^{c}$ & & $P<0.001^{c}$ & & \\
\hline \multicolumn{6}{|c|}{ Endothelial cell loss (\%) } \\
\hline 3 mo vs donor & $56 \pm 16$ & $31-80$ & $20 \pm 14$ & -14 to 59 & $<0.001$ \\
\hline 6 mo vs donor & $61 \pm 16$ & $25-83$ & $22 \pm 13$ & -7 to 45 & $<0.001$ \\
\hline 12 mo vs donor & $65 \pm 12$ & $23-81$ & $23 \pm 15$ & -14 to 69 & $<0.001$ \\
\hline
\end{tabular}

${ }^{\text {a }} P$ value between FLEK and PK.

${ }^{b} P<0.05$ vs donor endothelial cell density in a linear regression model.

' $P$ value of a linear regression model with three postoperative periods at the same time in the model.

FLEK, femtosecond laser-assisted endothelial keratoplasty; PK., penetrating keratoplasty; SD, standard deviation.

TABLE 4. Intraoperative and postoperative complications of FLEK and PK

\begin{tabular}{lll}
\hline & FLEK, $\mathbf{n}(\%)$ & PK, $\mathbf{n}(\%)$ \\
\hline Intraoperative pupillary block & $2(5.6 \%)$ & 0 \\
Postoperative pupillary block & $1(2.8 \%)$ & 0 \\
Primary graft failure & $3(8.3 \%)$ & 0 \\
Immunological graft failure & $1(2.8 \%)$ & 0 \\
Allograft rejection & 0 & $1(2.5 \%)$ \\
Suture-related problems & 0 & $4(10 \%)$ \\
Wound dehiscence & 0 & $2(5.0 \%)$ \\
Suture revision to correct astigmatism & 0 & $4(10 \%)$ \\
Elevated intraocular pressure & $5(13.9 \%)$ & $5(12.5 \%)$ \\
Dislocation and resposition of graft & $10(27.8 \%)$ & 0 \\
Epithelial ingrowth & $1(2.8 \%)$ & 0 \\
\hline
\end{tabular}

FLEK, femtosecond laser-assisted endothelial keratoplasty; PK, penetrating keratoplasty. 
Intraocular pressure elevation in the FLEK and PK groups occurred in 5 of the 36 eyes (13.9\%) and 5 of the 40 eyes (12.5\%), which was effectively treated with intraocular pressure lowering drops. In 6 of the 36 eyes (16.7\%) in the FLEK group, there was a noticeable central stromal haze after deturgescence of the recipient cornea.

\section{DISCUSSION}

Several studies have shown that EK techniques are effective procedures for treating eyes with endothelial disease and are becoming a popular alternative for PK (4-6). The purpose of this randomized multicenter trial was to evaluate the efficacy and safety of FLEK, where a femtosecond laser is used to prepare the endothelial graft, versus PK during a follow-up time of 12 months.

\section{Refractive outcome and visual outcome}

Our results show that both refractive and topographic astigmatism changed only in PK eyes but remained low and stable in FLEK eyes. Astigmatism after PK may be greater than $3.0 \mathrm{D}$ in more than $40 \%$ of eyes, leading to anisometropia, image distortion, and intolerance of spectacle wear (3, 22-25). Therefore, the important advantage of FLEK and other EK techniques over PK is the decreased induction of postoperative topographic and refractive astigmatism (26-28). Our study shows that the percentage of eyes with a refractive astigmatism more than or equal to $3 \mathrm{D}$ is significantly higher after PK. However, long-term follow-up remains necessary for full comparison of the final astigmatism results between FLEK and PK eyes, because most PK eyes still have all or most sutures in place at the 12-month time point.

Postoperative spherical equivalent was more hyperopic in the FLEK group, which may be due to the fact that the endothelial grafts prepared with the femtosecond laser are thinner in the center and thicker at the edges (12). The hyperopic shift in our study was higher compared with other EK studies (26-30), because we initially did not compensate for the expected shift by selecting a higher intraocular lens power, targeting at mild myopia (-1.0 to $-1.25 \mathrm{D})$ during cataract surgery (31).

We showed a significant improvement of UCVA in the FLEK group, with no significant difference between the FLEK and PK groups at 12 months. Recently, a randomized study demonstrated that UCVA in DLEK eyes improved over 12 months time, whereas no improvement was noted in PK eyes (32). Another nonrandomized study showed that UCVA was lowest in PK eyes compared with EK eyes (15).

We found a significant improvement of BSCVA in both FLEK and PK eyes with a significantly higher value in PK eyes. Our results of BSCVA in PK eyes are comparable with other PK studies $(3,15)$. The previously mentioned randomized study showed that 
BSCVA was comparable between DLEK and PK eyes over 1 year follow-up (32), whereas the nonrandomized comparative study reported that BSCVA was significantly better in DLEK, DSEK, and DSAEK eyes compared with PK eyes (15). However, the BSCVA results in our FLEK group seem to be lower compared with DSAEK series in the literature which report a mean BSCVA of 20/44 to 20/34 with a follow-up of 6 to 12 months $(4,15,28)$. Possible explanations for these difference are an increased opacification the interface due to keratocyte activation by the femtosecond laser or a suboptimal smoothness of the stromal bed as compared with a microkeratome-prepared bed $(16,33)$. To improve the smoothness of the stromal bed, it has been suggested to use a curved interface lens with the aim of reducing the circular concentric ridges (34). Furthermore, the $60-\mathrm{kHz}$ intralase femtosecond laser has been shown to produce a smoother anterior stromal bed in comparison to the microkerotome (35), but it is currently unclear what the effect will be on the smoothness when making deep lamellar cuts, to prepare the endothelial graft. Because there were significantly more eyes with age-related macular degeneration or retinal pigment epithelium changes in our FLEK group in comparison with the PK group we used a multiple linear regression model to adjust for age-related macular degeneration and retinal pigment epithelium changes. The analysis showed that the BSCVA at 3, 6, and 12 months was still significantly higher in the PK group.

Currently, there are four different femtosecond laser systems available, with different corneal applanation lenses and different frequencies. Previous studies with the FEMTEC femtosecond laser (FEMTEC, 20/10 Perfect Vision, Heidelberg, Germany) have reported a smooth stromal bed without circular concentric ridges when making a deep lamellar cut to prepare the endothelial graft $(11,13)$.

\section{Endothelial cell density}

An important goal of both EK and PK is to maintain a high endothelial cell density, because it is an essential factor for long-term graft survival. Endothelial cell loss in the first year after PK has been reported to vary between $24.0 \%$ and $39.8 \%$, which is comparable with our demonstrated endothelial cell loss of $23 \pm 15 \%$ in the PK group after 12 months follow-up $(36,37)$. Endothelial cell loss after small-incision DSEK and DSAEK has been reported to be between $34 \%$ and $61 \%$ at 12 months follow-up $(15,29,30,38,39)$. In our study, endothelial cell loss in the FLEK group at 12 months was $65 \pm 12 \%$, which is comparable with the high range of small-incision DSEK and DSAEK.

Over time, endothelial cell loss was significantly higher in the FLEK group compared with the PK group. The finding of endothelial cell loss is a concern for the long-term graft survival in EK eyes. FLEK is a challenging new surgical procedure, and the learning curve of various surgeons may have influenced the level of endothelial cell loss. Furthermore, several steps during the FLEK procedure such as the folding of the endothelial graft into a taco-configuration, squeezing of the endothelial graft by forceps during inser- 
tion, unfolding, and long-standing contact with air bubble may have influenced the postoperative endothelial cell density $(40,41)$. Recently, modified techniques have been proposed for inserting the endothelial graft during DSEK and DSAEK. Kaiserman et al. (14) have compared endothelial cell loss after insertion of the endothelial graft with a suture versus forceps, which showed no significant difference between the two groups. Other studies have used a glide to insert the endothelial graft and the endothelial cell loss varied between $23.5 \%$ and $25.3 \%$ at 6 and 12 months follow-up $(42,43)$.

\section{Intraoperative and postoperative complications}

In our study, no intraoperative complications occurred during PK. Two eyes (5.6\%) showed a pupillary block during FLEK. After the routine performance of a surgical iridectomy, no further cases of pupillary block glaucoma were seen. Only few intraoperative complications have been reported during EK, including donor tissue perforation (with hand-dissection), papillary block, and suprachoroidal hemorrhage $(27,44)$.

Postoperatively, three eyes in the FLEK group showed a primary graft failure. This was most likely related to the learning curve of various surgeons and several technical steps of the FLEK procedure. None of the PK eyes in this study demonstrated a primary graft failure. In the literature, the rate of primary graft failure after DSEK or DSAEK ranges from $0.5 \%$ to $45 \%(15,27,29)$.

One eye $(2.8 \%)$ in the FLEK demonstrated an immunological graft failure, and one eye $(2.5 \%)$ in the PK demonstrated an allograft rejection over time. The immunological graft failure and rejection rate in our study is similar or even lower than previous reports in non-high-risk case series for PK (4.4\%-29\%), DLEK (4\%-4.4\%), and DSAEK (2.2\%-9\%; $15,30,45)$. A 2-year follow-up study by Allan et al. (46) reported a lower rejection rate in the EK group (7.5\%) in comparison to the PK group (13\%). This can be explained by the fact that $80 \%$ of the EK patients were still using topical steroids and that most of the PK patients had discontinued their topical steroids 1 year postoperatively. In contrast, $19.4 \%$ of the patients in our FLEK group had discontinued their topical steroids after 6 months, and all patients in the PK group were still using topical steroids at 12 months follow-up. This demonstrates that FLEK-treated eyes may require only short-term steroid use and indicates that steroid-withdrawal does not necessarily lead to an increased rejection rate.

The incidence of steroid-induced glaucoma can be as high as $30 \%$ (47). The study by Bahar et al. (15) showed a rate of steroid-induced glaucoma of $6.3 \%$ in PK eyes, $13.2 \%$ in DLEK eyes, $12.5 \%$ in DSEK eyes, and $6.7 \%$ in DSAEK eyes. The incidence of steroidinduced glaucoma throughout our study (13.9\% in FLEK eyes and $12.5 \%$ in PK eyes) was comparable with previous reports. Currently, it is unclear which is the optimal time point for discontinuation of steroid drops in the FLEK group to decrease the risk of steroidinduced glaucoma even further. 
In our PK group, two eyes (5.0\%) showed a wound dehiscence (one eye due to a spontaneous wound dehiscence and the other due to blunt ocular trauma), which is comparable with previously reported incidences between $2.5 \%$ and $7.2 \%(48,49)$. After $\mathrm{PK}$, the eye is at risk for suture-related complications until all sutures are removed. Suture-related complications after PK are broken and loose sutures (8.3\%), infectious keratitis $(3.3 \%-50 \%)$, and erosions $(10.8 \% ; 48,49)$. In our PK group, four eyes (10\%) showed suture-related complications during the 12 months follow-up, which was comparable with these reports.

The most frequent complication in our FLEK series was graft dislocation $27.8 \%$ of eyes), which is comparable with the incidence in series of small-incision DSEK $(0.7 \%-$ $50.0 \%)$ and of DSAEK $(4.0 \%-34.6 \% ; 4,6,27,28,30,44)$. Patient selection for EK may be an important factor in reducing the rate of dislocation, for example, aphakic eyes with a floppy iris diaphragm after vitreoretinal surgery may not be suitable for EK procedures.

In our FLEK group, one eye (2.8\%) showed postoperative epithelial ingrowth in the interface, which probably occurred through the transcorneal incision. The ingrowth remained stable during the 12 months follow-up. This infrequent complication has been reported after both DSEK and DSAEK $(44,50,51)$.

In conclusion, this randomized trial with a follow-up of 12 months demonstrated the efficacy of both FLEK and PK for treating endothelial disease with a lower percentage of refractive astigmatism ( $\leq 3.0 \mathrm{D}$ ) and a mild hyperopic shift in FLEK eyes as compared with PK eyes. After 12 months follow-up, FLEK and PK showed an improvement of BSCVA, and the differences of BSCVA gain between the two groups were comparable. The major safety concern remains the high endothelial cell loss after FLEK compared with previous DSEK and DSAEK studies. Future research on FLEK should be directed toward refinements in donor tissue handling with the aim of minimizing endothelial cell loss and improvement of the interface optical quality by innovations in femtosecond laser technology. 


\section{REFERENCES}

1. ThompsonRWJr, Price MO, Bowers PJ, et al. Long-term graft survival after penetrating keratoplasty. Ophthalmology 2003; 110: 1396.

2. Williams KA, Esterman AJ, Bartlett C, et al. Howeffective is penetrating corneal transplantation? Factors influencing long-term outcome in multivariate analysis. Transplantation 2006; 81: 896.

3. Williams KA, Muehlberg SM, Lewis RF, et al. How successful is corneal transplantation? A report from the Australian Corneal Graft Register. Eye 1995; 9: 219.

4. Gorovoy MS. Descemet-stripping automated endothelial keratoplasty. Cornea 2006; 25: 886.

5. Terry MA, Ousley PJ. Deep lamellar endothelial keratoplasty in the first United States patients: Early clinical results. Cornea 2001; 20: 239.

6. Price FW Jr, Price MO. Descemet's stripping with endothelial keratoplasty in 200 eyes: Early challenges and techniques to enhance donor adherence. J Cataract Refract Surg 2006; 32: 411.

7. Melles GR, Eggink FA, Lander F, et al. A surgical technique for posterior lamellar keratoplasty. Cornea 1998; 17: 618.

8. Cheng YY, Pels E, Nuijts RM. Femtosecond-laser-assisted Descemet's stripping endothelial keratoplasty. J Cataract Refract Surg 2007; 33: 152.

9. Melles GR, Ong TS, Ververs B, et al. Preliminary clinical results of Descemet membrane endothelial keratoplasty. Am J Ophthalmol 2008; 145: 222.

10. Melles GR, Wijdh RH, Nieuwendaal CP. A technique to excise the Descemet membrane from a recipient cornea (descemetorhexis). Cornea 2004; 23: 286.

11. Seitz B, Langenbucher A, Hofmann-Rummelt C, et al. Nonmechanical posterior lamellar keratoplasty using the femtosecond laser (femtoplak) for corneal endothelial decompensation. Am J Ophthalmol 2003; 136: 769.

12. Cheng $Y Y$, Hendrikse $F$, Pels $E$, et al. Preliminary results of femtosecond laser-assisted Descemet stripping endothelial keratoplasty. Arch Ophthalmol 2008; 126: 1351.

13. Mehta JS, Shilbayeh R, Por YM, et al. Femtosecond laser creation of donor cornea buttons for Descemet-stripping endothelial keratoplasty. J Cataract Refract Surg 2008; 34: 1970.

14. Kaiserman I, Bahar I, McAllum P, et al. Suture-assisted vs forceps-assisted insertion of the donor lenticula during Descemet stripping automated endothelial keratoplasty. Am J Ophthalmol 2008; 145: 986.

15. Bahar I, Kaiserman I, McAllum P, et al. Comparison of posterior lamellar keratoplasty techniques to penetrating keratoplasty. Ophthalmology 2008; 115: 1524.

16. Jones YJ, Goins KM, Sutphin JE, et al. Comparison of the femtosecond laser (IntraLase) versus manual microkeratome (Moria ALTK) in dissection of the donor in endothelial keratoplasty: Initial study in eye bank eyes. Cornea 2008; $27: 88$.

17. Eye Bank Association of America. 2007 Eye Banking Statistical Report. Washington, DC, Eye Bank Association of America 2007.

18. Cheng YY, Kang SJ, Grossniklaus HE, et al. Histologic evaluation of human posterior lamellar discs for femtosecond laser Descemet's stripping endothelial keratoplasty. Cornea 2009; 28: 73.

19. Pels E, Schuchard Y. Organ-culture preservation of human corneas. Doc Ophthalmol 1983; 56: 147.

20. Cheng YY, Pels E, Cleutjens JP, et al. Corneal endothelial viability after femtosecond laser preparation of posterior lamellar discs for Descemet-stripping endothelial keratoplasty. Cornea 2007; 26: 1118.

21. Ferris FL III, Kassoff A, Bresnick GH, et al. New visual acuity charts for clinical research. Am J Ophthalmol 1982; 94: 91. 
22. Frost NA, Wu J, Lai TF, et al. A review of randomized controlled trials of penetrating keratoplasty techniques. Ophthalmology 2006; 113: 942.

23. The Australian Corneal Graft Registry. 1990 to 1992 report. Aust NZ J Ophthalmol 1993; $21: 1$.

24. RiddleHKJr, Parker DA, PriceFWJr. Management of postkeratoplasty astigmatism. Curr Opin Ophthalmol 1998; 9: 15.

25. Brooks SE, Johnson D, Fischer N. Anisometropia and binocularity. Ophthalmology 1996; 103: 1139.

26. Koenig SB, Covert DJ. Early results of small-incision Descemet's stripping and automated endothelial keratoplasty. Ophthalmology 2007; 114: 221.

27. Price MO, Price FW Jr. Descemet's stripping with endothelial keratoplasty: Comparative outcomes with microkeratome-dissected and manually dissected donor tissue. Ophthalmology 2006; 113: 1936.

28. Covert DJ, Koenig SB. New triple procedure: Descemet's stripping and automated endothelial keratoplasty combined with phacoemulsification and intraocular lens implantation. Ophthalmology 2007; 114: 1272.

29. Mearza AA, Qureshi MA, Rostron CK. Experience and 12-month results of Descemet-stripping endothelial keratoplasty (DSEK) with a small-incision technique. Cornea 2007; 26: 279.

30. Koenig SB, Covert DJ, Dupps WJ Jr, et al. Visual acuity, refractive error, and endothelial cell density six months after Descemet stripping and automated endothelial keratoplasty (DSAEK). Cornea 2007; 26: 670.

31. Holz HA, Meyer JJ, Espandar L, et al. Corneal profile analysis after Descemet stripping endothelial keratoplasty and its relationship to postoperative hyperopic shift. J Cataract Refract Surg 2008; 34: 211.

32. Patel SV, McLaren JW, Hodge DO, et al. Scattered light and visual function in a randomized trial of deep lamellar endothelial keratoplasty and penetrating keratoplasty. Am J Ophthalmol 2008; 145: 97.

33. Hindman HB, McCally RL, Myrowitz E, et al. Evaluation of deep lamellar endothelial keratoplasty surgery using scatterometry and wavefront analyses. Ophthalmology 2007; 114: 2006.

34. Soong HK, Mian S, Abbasi O, et al. Femtosecond laser-assisted posterior lamellar keratoplasty: Initial studies of surgical technique in eye bank eyes. Ophthalmology 2005; 112: 44.

35. Sarayba MA, Ignacio TS, Tran DB, et al. A $60 \mathrm{kHz}$ IntraLase femtosecond laser creates a smoother LASIK stromal bed surface compared to a Zyoptix XP mechanical microkeratome in human donor eyes. J Refract Surg 2007; 23: 331.

36. Patel SV, Hodge DO, Bourne WM. Corneal endothelium and postoperative outcomes 15 years after penetrating keratoplasty.AmJ Ophthalmol 2005; 139: 311.

37. Ing JJ, Ing HH, Nelson LR, et al. Ten-year postoperative results of penetrating keratoplasty. Ophthalmology 1998; 105: 1855.

38. Terry MA, Chen ES, Shamie N, et al. Endothelial cell loss after Descemet's stripping endothelial keratoplasty in a large prospective series. Ophthalmology 2008; 115: 488.

39. Price MO, Price FW Jr. Endothelial cell loss after Descemet stripping with endothelial keratoplasty influencing factors and 2-year trend. Ophthalmology 2008; 115: 857.

40. Ide T, Yoo SH, Goldman JM, et al. Descemet-stripping automated endothelial keratoplasty: Effect of inserting forceps on DSAEK donor tissue viability by using an in vitro delivery model and vital dye assay. Cornea 2007; 26: 1079.

41. Olson RJ. Air and the corneal endothelium: An in vivo specular microscopy study in cats. Arch Ophthalmol 1980; 98: 1283. 
42. Mehta JS, Por YM, Poh R, et al. Comparison of donor insertion techniques for Descemet stripping automated endothelial keratoplasty. Arch Ophthalmol 2008; 126: 1383.

43. Busin M, Bhatt PR, Scorcia V. A modified technique for Descemet membrane stripping automated endothelial keratoplasty to minimize endothelial cell loss. Arch Ophthalmol 2008; 126: 1133.

44. Suh LH, Yoo SH, Deobhakta A, et al. Complications of Descemet's stripping with automated endothelial keratoplasty: Survey of 118 eyes at One Institute. Ophthalmology 2008; 115: 1517.

45. Jonas JB, Rank RM, Budde WM. Immunologic graft reactions after allogenic penetrating keratoplasty. Am J Ophthalmol 2002; 133: 437.

46. Allan BD, Terry MA, Price FW Jr, et al. Corneal transplant rejection rate and severity after endothelial keratoplasty. Cornea 2007; 26: 1039.

47. Jones R III, Rhee DJ. Corticosteroid-induced ocular hypertension and glaucoma: A brief review and update of the literature. Curr Opin Ophthalmol 2006; 17: 163.

48. Rehany U, Rumelt S. Ocular trauma following penetrating keratoplasty: Incidence, outcome, and postoperative recommendations. Arch Ophthalmol 1998; 116: 1282.

49. Abou-Jaoude ES, Brooks M, Katz DG, et al. Spontaneous wound dehiscence after removal of single continuous penetrating keratoplasty suture. Ophthalmology 2002; 109: 1291.

50. Culbertson WW. Descemet stripping endothelial keratoplasty. Int Ophthalmol Clin 2006; $46: 155$.

51. Koenig SB, Covert DJ. Epithelial ingrowth after Descemet-stripping automated endothelial keratoplasty. Cornea 2008; 27: 727. 



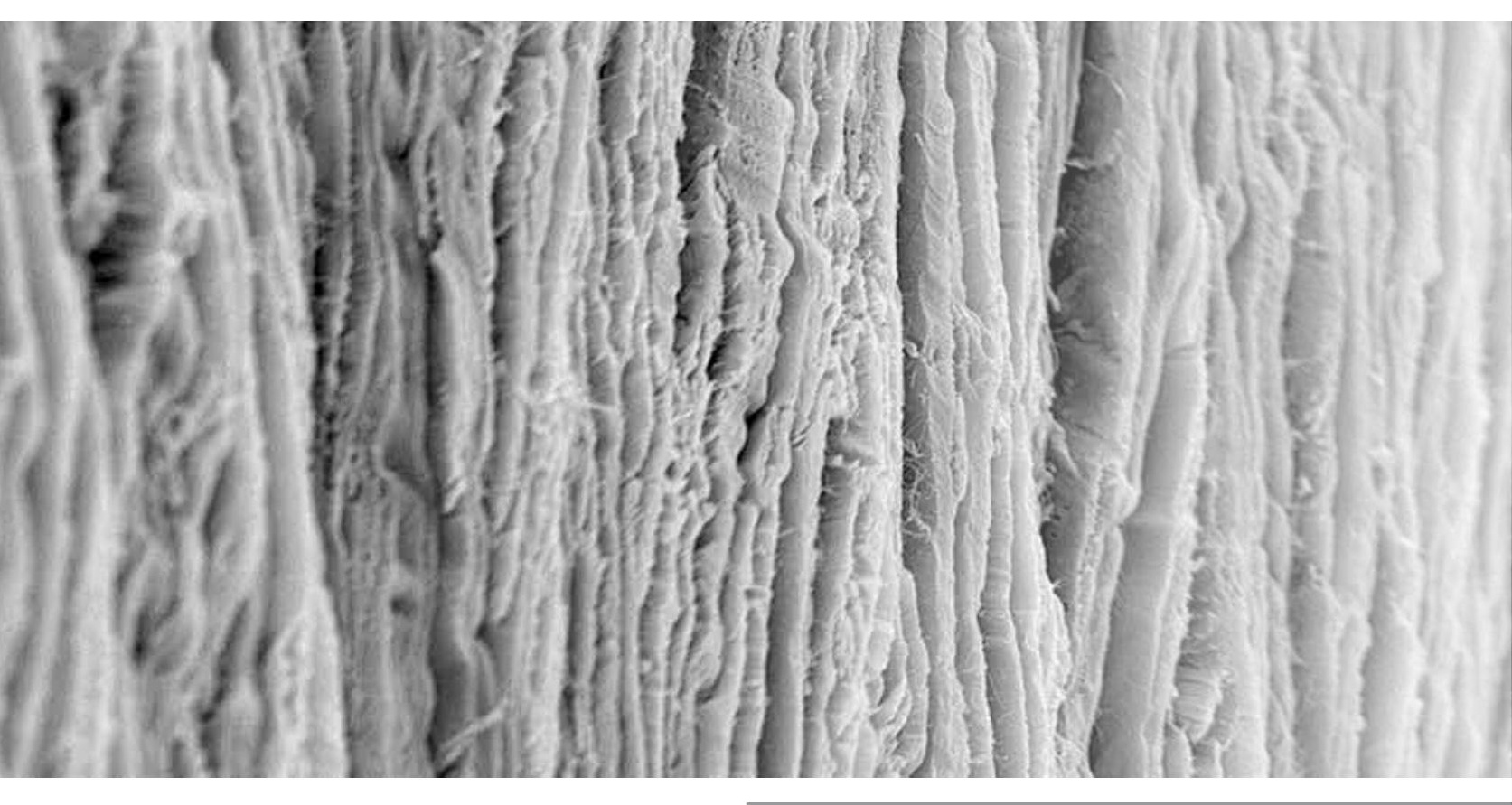




\title{
CHAPTER 7
}

Quality of Vision After

Femtosecond Laser-Assisted

Descemet Stripping Endothelial

Keratoplasty and Penetrating

Keratoplasty: A Randomized,

Multicenter Clinical Trial

\author{
Yanny Y.Y. Cheng \\ Tom J.T.P. van den Berg \\ Jan S. Schouten \\ Elisabeth Pels \\ Robert-Jan Wijdh \\ Hugo van Cleynenbreugel \\ Catharina A. Eggink, \\ Wilhemina J. Rijneveld \\ Rudy M.M.A. Nuijts
}

Am J Ophthalmol. 2011 Oct;152(4):556-66 


\section{ABSTRACT}

Purpose: To compare the quality of vision (straylight and contrast sensitivity) after femtosecond laser-assisted Descemet stripping endothelial keratoplasty (FS DSEK) and penetrating keratoplasty (PK).

Design: Prospective, randomized clinical trial.

Methods: Setting: Multicenter (5 ophthalmic centers in The Netherlands).

Study population: Eighty eyes of 80 patients with corneal endothelial dysfunction were included and were randomized to FS DSEK or PK.

Observation procedures: FS DSEK and PK.

Main outcome measures: Straylight, contrast sensitivity, astigmatism, uncorrected visual acuity, best spectacle-corrected visual acuity (BSCVA), and visual symptom score.

Results: Straylight at 12 months was $1.37 \pm 0.2$ logarithm of straylight for FS DSEK and $1.46 \pm 0.2$ logarithm of straylight for PK $(P=.151)$. During 12 months of follow-up, there was a significant improvement of straylight and contrast sensitivity after FS DSEK $(P<.001)$ and PK $(P<.001)$. The change of straylight and contrast sensitivity correlated significantly with the change of BSCVA after FS DSEK $(r=-0.645 ; r=0.580)$ and PK $(r=$ $-0.370 ; r=0.659$ ). The visual symptom score was comparable between the 2 groups during the 12 months of follow-up.

Conclusions: Improvement of straylight and contrast sensitivity was significantly correlated with an improvement of BSCVA. Straylight and contrast sensitivity were improved significantly after FS DSEK and were comparable with those after PK, although BSCVA was slightly better in the PK group. 


\section{INTRODUCTION}

Penetrating keratoplasty (PK) has been shown to be a successful treatment for restoring vision in eyes with corneal endothelial disease, but disadvantages include a slow visual rehabilitation, high irregular astigmatism, and suture-related problems. ${ }^{1-3}$ In 1956, the first posterior lamellar keratoplasty was performed to replace the deep stromal and endothelial layers and to maintain the anterior part of the cornea. ${ }^{4}$ Posterior lamellar keratoplasty was not performed regularly because of difficulties with surgical techniques. After major surgical improvements and innovations in surgical instruments, endothelial keratoplasty replaced PK as the gold standard surgical technique for corneal endothelial disease. $^{5-10}$

Major advantages of endothelial keratoplasty, compared with PK, are a minimal change in corneal astigmatism, a more predictable postoperative spherical equivalent, and a stable globe more resistant to trauma. ${ }^{6,8,9}$ In a recent randomized clinical trial of femtosecond laser-assisted Descemet stripping endothelial keratoplasty (FS DSEK) versus PK, we showed that FS DSEK effectively reduces postoperative astigmatism, but we also showed a lower visual acuity as compared with conventional PK. ${ }^{11}$

We hypothesized that the lower visual acuity may be the result of the formation of interface haze at the donor-recipient stromal interface. This haze may result in an increase of intraocular straylight and a decrease of contrast sensitivity. ${ }^{12,13}$ Consequently, a dissatisfaction with the quality of vision and limitations in daily functioning may occur. ${ }^{14-17}$

This randomized clinical trial was performed to determine intraocular straylight, contrast sensitivity, and subjective visual symptoms after FS DSEK and PK and to correlate these quality-of-vision parameters to the refractive and visual outcomes. A secondary goal of the present study was to test the clinical use of straylight meter in such a demanding setting.

\section{METHODS}

This randomized, multicenter trial was conducted at 5 ophthalmic centers in The Netherlands. Inclusion criteria were endothelial dysfunction caused by Fuchs endothelial dystrophy, aphakic or pseudophakic bullous keratopathy or posterior polymorphous dystrophy, a minimal age of 18 years, and a best spectacle-corrected visual acuity (BSCVA) lower than 20/50. Patients were excluded if they had undergone previous PK, had human leukocyte antigen typed keratoplasty, or were mentally retarded. The medical history was recorded, and all patients underwent a slit-lamp examination. Preoperative collected data included patient age, gender, refractive error, preoperative lens status, and ocular comorbidities. 


\section{Surgical procedures}

The surgical techniques of FS DSEK and PK have been described previously. ${ }^{11}$

\section{Outcomes measures}

The primary outcome measures were straylight and contrast sensitivity. Secondary outcome measures included refractive astigmatism, topographic astigmatism, uncorrected visual acuity (UCVA), BSCVA, and visual symptom score. All outcome measures were measured before surgery and at 3,6, and 12 months of follow-up.

Straylight was measured using a straylight meter (CQuant; Oculus $\mathrm{GmbH}$, Wetzlar, Germany), which uses a compensation comparison method with a forced-choice technique. ${ }^{18}$ Clinical straylight measurement is a relatively new development to quantify quality of vision and was developed originally for visual acuities better than 0.7 logarithm of the minimal angle of resolution (logMAR). However, corneal transplantation patients often have visual acuities worse than that.

The straylight value was expressed as a logarithmic intraocular straylight $(\log (\mathrm{s}))$ value. Higher values indicate more straylight and an increased sensitivity to glare. ${ }^{19}$ Two consecutive straylight measurements of the study eye and the nonstudy eye were obtained, after which an average amount of logarithmic intraocular straylight was calculated. The instrument derives a reliability value for each measurement, called the expected standard deviation, on the basis of known psychometric principles. A reliable value was defined as an expected standard deviation of less than 0.08 log units. The repeated measures design of the study checked this reliability estimate against true reliability. Straylight values also were compared with a control group obtained from a previous database consisting of agematched subjects with a clear cornea and no cataract. ${ }^{19}$ Eyes that were unable to perform the straylight test before surgery were substituted by the highest preoperative log(s) plus 0.1 .

The contrast sensitivity was measured using the Pelli-Robson chart (Clement Clarke Ltd, Harlow, United Kingdom). This chart was chosen from among other available charts for its high reliability and validity compared with sinusoidal grating charts such as the Vistech and Functional Acuity Contrast Test. ${ }^{20-22}$ Patients were tested both monocularly and binocularly, using the best spectacle correction for distance vision on a testing distance of $1 \mathrm{~m}$ and a luminance of 85 candelas $/ \mathrm{m}^{2}$. The last triplet of letters, of which at least 2 letters were seen correctly, was recorded and expressed as a logarithmic contrast sensitivity value. ${ }^{23}$ Lower values indicate a better contrast sensitivity.

Topographic astigmatism was measured using the EyeMap corneal topographer (EH-290; Alcon, Fort Worth, Texas, USA). The UCVA and BSCVA were determined using the Early Treatment Diabetic Retinopathy Study letter charts and were converted to logarithm of the minimal angle of resolution measurements. ${ }^{24}$ Vision levels of counting 
fingers, hand movements, light perception, and no light perception were substituted by logarithm of the minimal angle of resolution values of 1.7, 2.0, 2.5, and 3.0, respectively.

Double-vision or distorted vision, glare, halo, blurry vision, and differently looking colors were reported by patients using a validated questionnaire. ${ }^{25}$ The grading of symptoms ranged from great deal, moderate amount, little, to none, and a score of 3, 2, 1 , or 0 was assigned, respectively. Scores for each of the symptoms then were summed, and this resulted in a visual symptom score ranging from 0 (not at all bothered by any of the symptoms) to 15 (very bothered by all symptoms). ${ }^{25,26}$

\section{Sample size}

The sample size calculation of the main outcome of this randomized clinical trial has been described previously. ${ }^{11}$

\section{Randomization}

All included eyes were assigned randomly to either the FS DSEK or the PK group. The randomization code was generated using a permuted block size of 2 . The assigned treatment plans then were sent to the surgeon.

\section{Statistical analysis}

Data were described as mean standard deviation for continuous variables and as individual counts and percentages for categorical variables. Differences between groups were analyzed using a Student $t$ test for continuous data. The Pearson chi-square test was used to compare categorical data. Comparisons of preoperative data and postoperative data within a group were performed using a linear regression model. Correlations were assessed using the Pearson correlation coefficient in case of normal distributed data and using the Spearman test in case of abnormal distributed data. A $P$ value of less than .05 was considered to be statistically significant. Statistical analysis was performed using SPSS software for Windows version 15.0 (SPSS, Inc, Chicago, Illinois, USA).

\section{RESULTS}

\section{Participant flow chart}

Eighty eyes of 80 patients were recruited, with 40 eyes in each arm (Figure 1). In the FS DSEK group, 4 patients did not receive the allocated treatment because of significant preoperative events (such as keratitis, corneal ulcers, or both) and eventually were excluded from the study analysis. All patients in the PK group received the allocated treatment. 


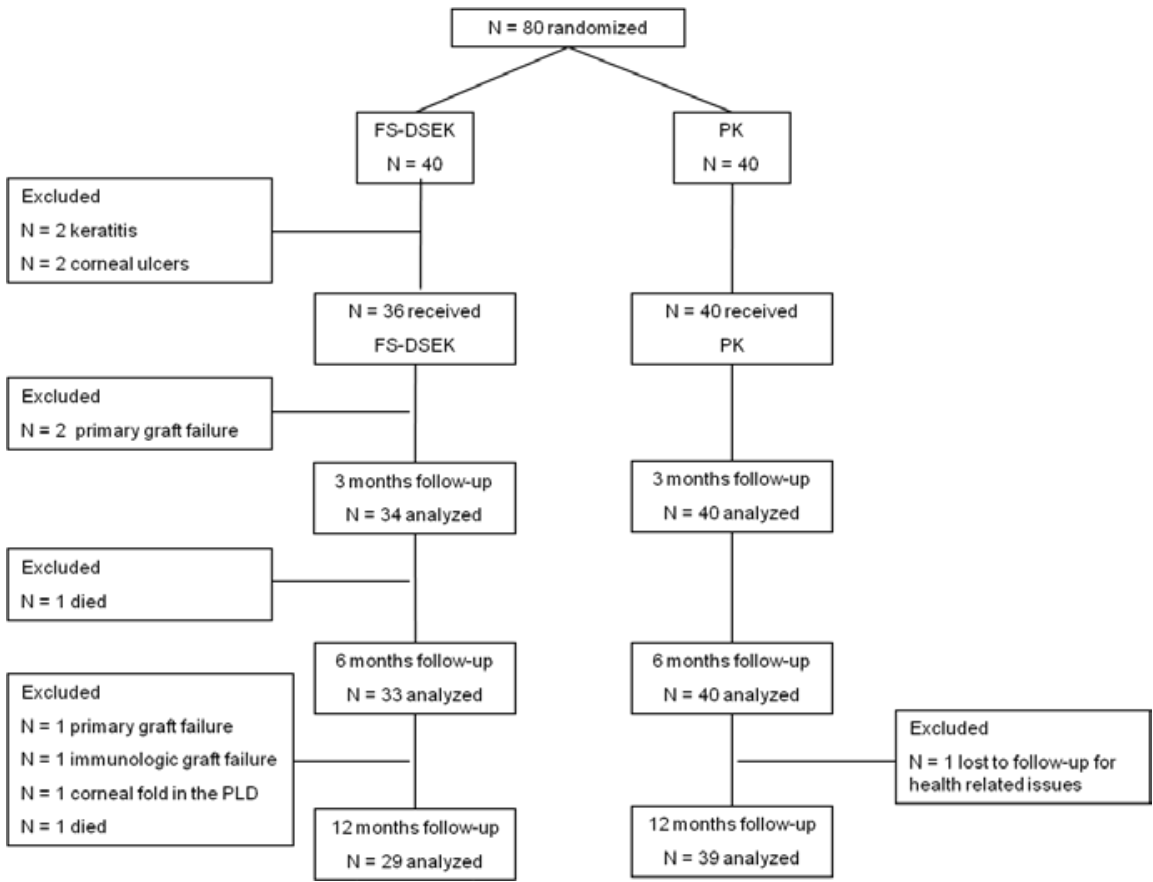

FIGURE 1. Participant flow chart of femtosecond laser-assisted Descemet stripping endothelial keratoplasty versus penetrating keratoplasty. FS-DSEK = femtosecond laser-assisted Descemet stripping endothelial keratoplasty; $\mathrm{PK}=$ penetrating keratoplasty; PLD = posterior lamellar disc.

In the FS DSEK group, 29 eyes were available for analysis at the 12-month follow-up. After surgery, the cornea of 3 eyes remained edematous and did not clear up; this was defined as primary graft failure. Two eyes underwent PK before the 3-month follow-up, and 1 eye underwent repeat FS DSEK after 6 months of follow-up.

\section{Patient characteristics}

Patients characteristics of the FS DSEK and PK group are listed in Table 1. The mean age of the FS DSEK group and PK group was $69.0 \pm 8.8$ years and $71.4 \pm 11.3$ years, respectively $(P=.308)$. In the FS DSEK group, 21 of 36 patients (58.3\%) were diagnosed with Fuchs endothelial dystrophy, and $8(38.1 \%)$ of these 21 patients also had visually significant cataract. These patients either underwent primary cataract extraction with intraocular lens (IOL) implantation ( $n=5,62.5 \%)$ followed by the FS DSEK procedure or a combined procedure of FS DSEK and cataract extraction with IOL implantation ( $n=3,37.5 \%)$. In the PK group, 20 of 40 patients (50.0\%) were diagnosed with Fuchs endothelial dystrophy, and $12(60.0 \%)$ of these 20 patients also had visually significant cataract. Ten (83.3\%) of these 12 patients underwent a combined procedure of PK and cataract extraction 
TABLE 1. Preoperative Patient Characteristics of Femtosecond Laser-Assisted Descemet Stripping Endothelial Keratoplasty and Penetrating Keratoplasty

\begin{tabular}{|c|c|c|c|}
\hline & FS-DSEK & PK & $P$ Value \\
\hline Eyes (n) & 36 & 40 & NA \\
\hline Mean age \pm SD $(y)$ & $69.0 \pm 8.8$ & $71.4 \pm 11.3$ & .308 \\
\hline No. women (\%) & $21(58.3 \%)$ & $27(67.5 \%)$ & .500 \\
\hline Diagnosis & & & .725 \\
\hline Fuchs' endothelial dystrophy & $21(58.3 \%)$ & $20(50.0 \%)$ & \\
\hline Pseudophakic bullous keratopathy & $15(41.7 \%)^{a}$ & $19(47.5 \%)^{\mathrm{a}}$ & \\
\hline Posterior polymorphous dystrophy & 0 & $1(2.5 \%)$ & \\
\hline Recipient lens status & & & .996 \\
\hline Aphakic & $1(2.8 \%)^{a}$ & $1(2.5 \%)^{a}$ & \\
\hline Phakic & $21(58.3 \%)$ & $21(52.5 \%)$ & \\
\hline Pseudophakic & $14(38.9 \%)$ & $18(45.0 \%)$ & \\
\hline \multicolumn{4}{|l|}{ Ocular comorbidity } \\
\hline Age-related macular degeneration/ RPE changes & $12(33.3 \%)$ & $5(12.5 \%)$ & .030 \\
\hline Cataract & $8(22.2 \%)$ & $12(30.0 \%)$ & .442 \\
\hline Glaucoma & $1(2.8 \%)$ & $3(7.5 \%)$ & .357 \\
\hline
\end{tabular}

FS DSEK = femtosecond laser-assisted Descemet stripping endothelial keratoplasty; $\mathrm{NA}=$ not applicable; $\mathrm{PK}=$ penetrating keratoplasty; RPE = retinal pigment epithelium; $\mathrm{SD}=$ standard deviation.

${ }^{a}$ One aphakic eye with iris-fixated anterior chamber intraocular lens.

with IOL implantation, and 2 of the 12 patients (16.7\%) underwent a primary cataract extraction with IOL implantation before PK.

Before surgery, 34 patients (85.0\%) in the PK group and 30 patients (83.3\%) in the FS DSEK group required spectacle correction for distance vision; the remaining patients used no correction. Twelve months after FS DSEK, 23 patients (79.3\%) used spectacles and 1 patient (3.4\%) used soft contact lenses for distance vision. Five patients (17.2\%) did not need a correction. Twelve months after PK, 26 patients (66.6\%) used spectacles, 1 patient (2.6\%) used a rigid contact lenses for distance vision, and 12 patients (30.8\%) did not use a correction, with 7 of the 12 patients being unable to wear a correction because of anisometropia.

\section{Intaocular straylight}

Before surgery, $43 \%$ of subjects had a visual acuity lower than the advised limit for straylight measurement (0.7 logMAR). This limit seems a bit strict, because only 11 (30.6\%) of 36 patients in the FS DSEK group and 13 (32.5\%) of 40 patients in the PK group were unable to complete the straylight test. The mean logMAR BSCVA of these patients' eyes was significantly higher in comparison with eyes of patients who did complete the test 


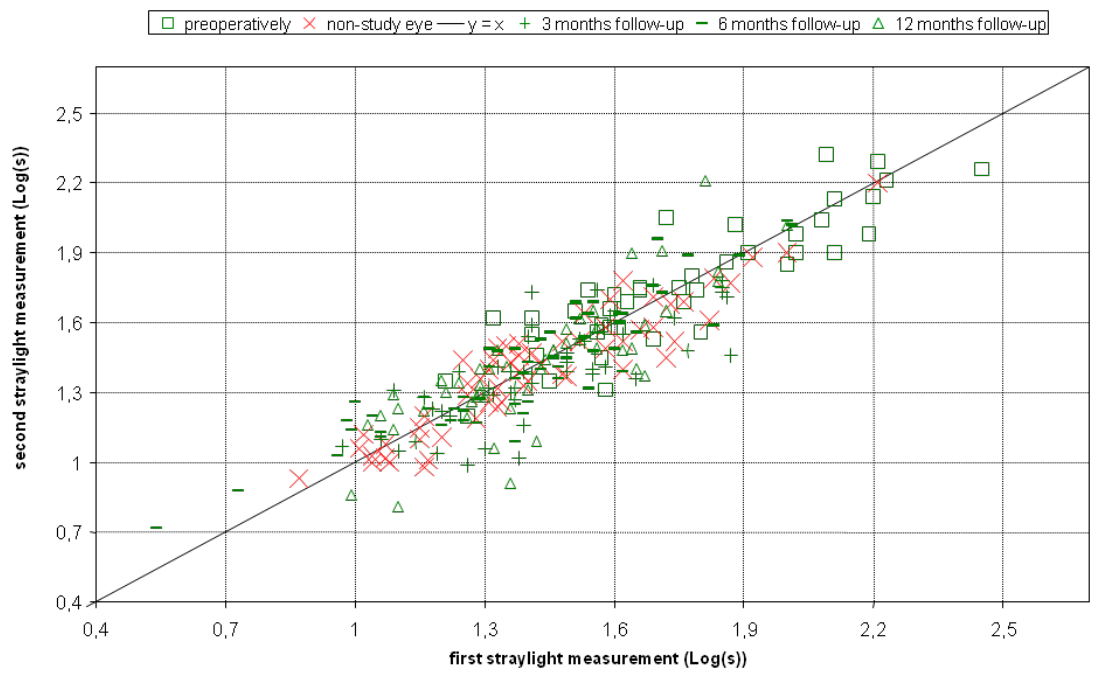

FIGURE 2. Graph showing the repeatability of intraocular straylight measurement for femtosecond laser-assisted Descemet stripping endothelial keratoplasty, penetrating keratoplasty, and nonstudy eyes. The repeated measures standard deviation was 0.07 for the nonstudy eyes and 0.10 overall for the study eyes. Log(s) = logarithmic intraocular straylight value.

$(1.17 \pm 0.5 \log M A R$ vs $0.67 \pm 0.2 \log M A R$, respectively $[P<.001]$, in the FS DSEK group; and $1.00 \pm 0.5 \log M A R$ vs $0.59 \pm 0.3 \log M A R$, respectively $[P<.002]$, in the PK group). Figure 2 shows repeatability of the straylight measurement for all follow-up visits of the study eyes and for the nonstudy eyes ( $P<.05$ for all 4 comparisons, $\mathrm{F}$ test). The repeated-measures standard deviation was 0.07 for the nonstudy eyes. For the study eyes, the repeated-measures standard deviations were slightly higher at 0.10, 0.09, 0.09, and 0.11 for preoperative and postoperative values at 3, 6, and 12 months, respectively. So, the precision of the straylight measurements was not much less in the study eyes as compared with the nonstudy eyes, but the difference was statistically significant $(P<.05$ for all comparisons, F test). Repeatability was not dependent on straylight level, is is also evident in Figure 2.

Preoperative straylight values were not significantly different between the FS DSEK and PK group (1.97 $\pm 0.4 \log (\mathrm{s})$ vs $1.97 \pm 0.4 \log (\mathrm{s})$, respectively; $P=.926)$. During the follow-up, the straylight between the FS DSEK and PK group was comparable (3 months, $1.43 \pm 0.2 \log (\mathrm{s})$ vs $1.40 \pm 0.2 \log (\mathrm{s})[P=.582] ; 6$ months, $1.42 \pm 0.3 \log (\mathrm{s})$ vs $1.41 \pm 0.2$ $\log (\mathrm{s})[P=.960] ; 12$ months, $1.37 \pm 0.2$ vs $1.46 \pm 0.2 \log (\mathrm{s})[P=.151])$. In both groups, there was a significant improvement of straylight during the 12 months of follow-up (FS DSEK, $P<.001 ;$ PK, $P<.001)$.

Straylight value as a function of age is shown in Figure 3. Eyes with too severe corneal edema that were not able to complete the straylight test were substituted by $2.46 \log (\mathrm{s})$, 
which is notable in Figure 3. Before surgery, $13.9 \%(n=5)$ of the patients in the FS DSEK group and $12.5 \%(n=5)$ of the patients in the PK group had straylight values within the normal age-matched range, whereas the remaining patients had higher straylight values. Twelve months after FS DSEK and PK, 38.5\% $(n=10)$ and $45.7 \%(n=16)$ of the patients, respectively, had straylight values comparable with the normal age-matched range, and $11.5 \%(n=3)$ and $2.9 \%(n=1)$ of the patients, respectively, had lower straylight values, whereas $50.0 \%(n=13)$ and $51.4 \%(n=18)$ of the patients, respectively, had higher straylight values.

Before surgery, there was a significant correlation between the BSCVA and straylight value in the FS DSEK group $(r=0.461 ; P=.005)$ and PK group $(r=0.523 ; P=.001)$. At 3,6 , and 12 months after surgery, the correlation between BSCVA and straylight value was not significant in the FS DSEK group $(r=0.260, P=.209 ; r=0.214, P=.273$; and $r=$ $0.082, P=.696$, respectively). In the PK group, the BSCVA was correlated significantly with straylight at 3,6 , and 12 months of follow-up $(r=0.363, P=.032 ; r=0.492, P=.003$; and $r=0.569, P<.001$ ).

The change in intraocular straylight and BSCVA values from baseline to 12 months after surgery showed a correlation in the PK group ( $r=-0.370 ; P=.029)$, and in the FS DSEK group $(r=-0.645 ; P<.001)$. An improvement of BSCVA was correlated significantly with a decrease of straylight in both groups.

\section{Contrast sensitivity}

Contrast sensitivities for both groups are shown in Table 2. Before surgery, 3 (8.3\%) of 36 patients in the FS DSEK group and 3 (7.5\%) of 40 patients in the PK group were unable to see the highest contrast at $1 \mathrm{~m}$ distance. Before surgery, contrast sensitivity of the study eye, fellow eye, and binocularly were not significantly different between the FS DSEK and PK groups.

At 3, 6, and 12 months after surgery, no significant difference in contrast sensitivity was found between the FS DSEK and PK groups. In both the FS DSEK and PK groups, contrast sensitivity of the study eye increased significantly after surgery. The binocular contrast sensitivity improved significantly after PK $(P=.006)$, but not after FS DSEK $(P=$ .365).

Before surgery, there was a correlation between BSCVA and contrast sensitivity in both the FS DSEK group $(r=-0.640 ; P<.001)$ and PK group $(r=-0.706 ; P<.001)$. After FS DSEK and $P K$, the change in contrast sensitivity from baseline to 12 months after surgery showed a correlation with the change in BSCVA during the same follow-up period $(r=$ $0.508, P=.009$, and $r=0.659, P<.001$, respectively).

In the FS DSEK group, the correlation between straylight and contrast sensitivity was $r=-0.504$ (preoperative, $P=.003$ ), $r=-0.541$ (3-month postoperative, $P=.005), r=-0.685$ (6-month postoperative, $P<.01$ ), and $r=-0.054$ (12-month postoperative, $P=.796)$. In 

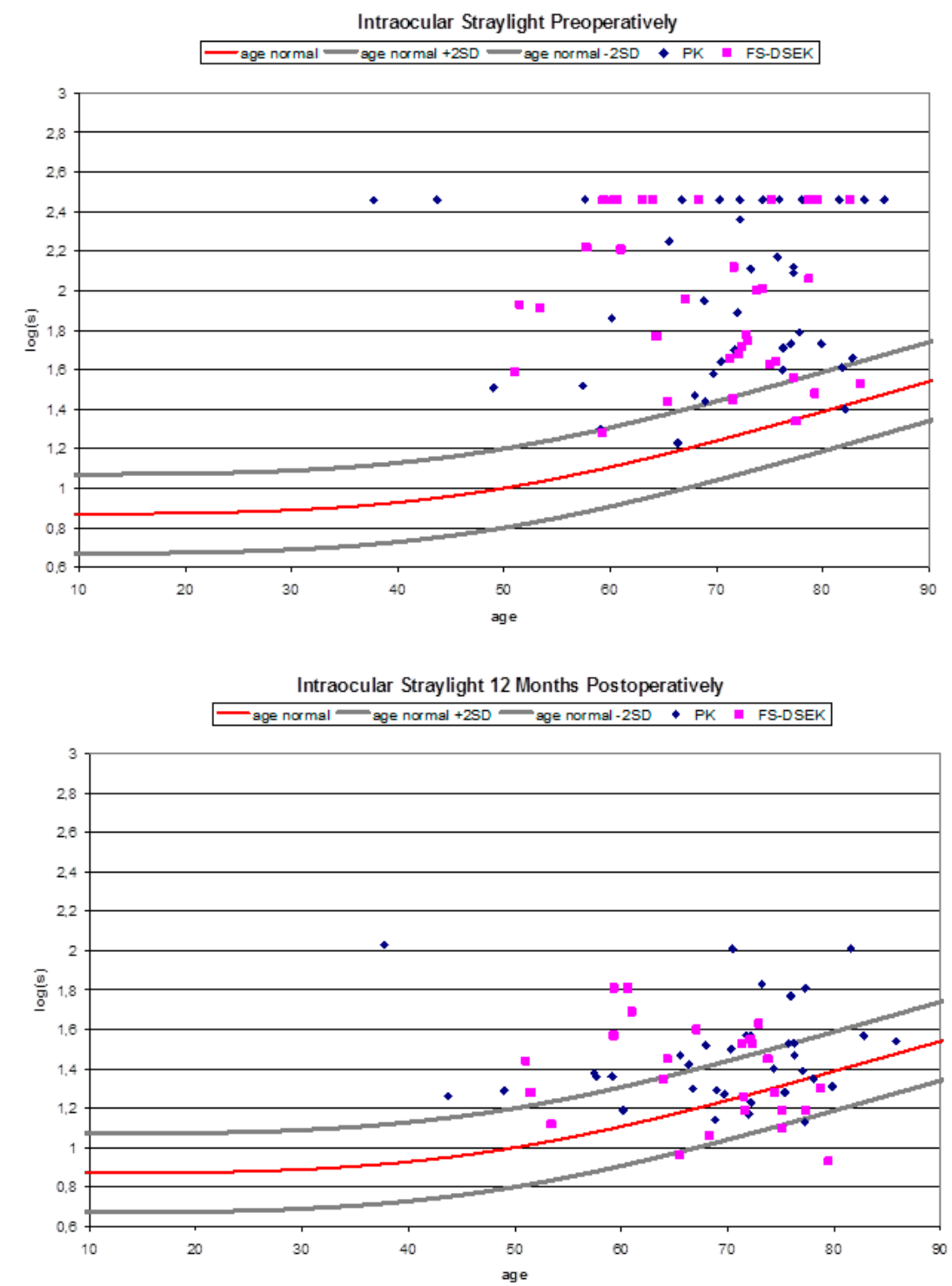

FIGURE 3. Graphs showing intraocular straylight values as a function of age for the femtosecond laser-assisted Descemet stripping endothelial keratoplasty (FS-DSEK) versus penetrating keratoplasty (PK) groups (Left) before surgery and (Right) 12 months after surgery. The lines represent mean levels of straylight \pm 2 standard deviations (SDs). Log(s) = logarithmic intraocular straylight value. 


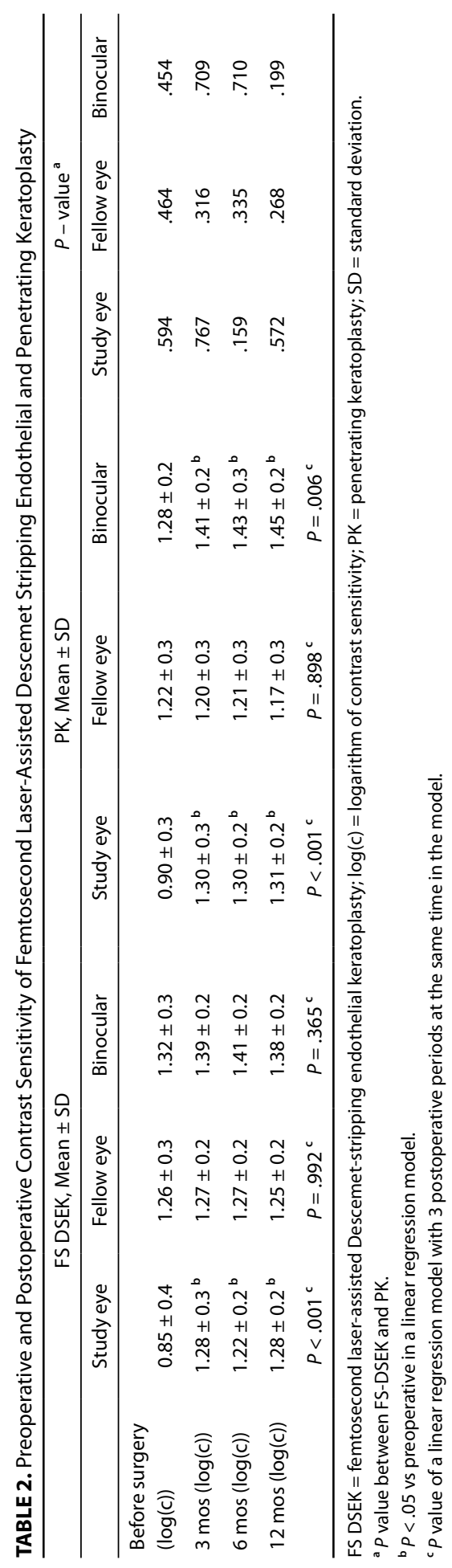


the PK group, the correlation between straylight and contrast sensitivity was $r=-0.434$ (preoperative, $P=.007$ ), $\mathrm{r}=-0.492$ (3-month postoperative, $P=.003$ ), $\mathrm{r}=-0.534$ (6-month postoperative, $P=.001$ ), and $r=-0.348$ (12-month postoperative, $P=.040$ ).

\section{Astigmatism}

Refractive and topographic astigmatism outcomes are shown in Table 3. Before surgery, there was no significant difference in refractive and topographic astigmatism between the FS DSEK and PK group. At the 12-month follow-up, both refractive and topographic astigmatism were significantly higher in the PK group compared with the FS DSEK group (refractive, -2.98 diopters [D] vs $-1.22 \mathrm{D}$, respectively; and topographic, $3.67 \mathrm{D}$ vs $1.58 \mathrm{D}$, respectively). In the FS DSEK group, postoperative refractive and topographic astigmatism values were not significantly different from preoperative values. In the PK group, all postoperative refractive and topographic astigmatism values were significantly higher compared with those before surgery. During follow-up, these values showed a tendency to decrease. After 12 months of follow-up, all sutures had been removed in only 1 eye (2.5\%) in the PK group.

Twelve months after surgery, the percentage of patients with a refractive astigmatism of $\leq 3.0 \mathrm{D}$ was significantly higher in the FS DSEK group compared with the PK group (86.2\% vs $51.3 \%$, respectively; $P=.004)$.

TABLE 3. Preoperative and Postoperative Astigmatism of Femtosecond Laser-Assisted Descemet-Stripping Endothelial Keratoplasty and Penetrating Keratoplasty

\begin{tabular}{|c|c|c|c|c|c|}
\hline & FS DSEK, Mean \pm SD & Range & $\mathrm{PK}$, Mean $\pm \mathrm{SD}$ & Range & $P$ Value $^{\text {a }}$ \\
\hline \multicolumn{6}{|c|}{ Refractive astigmatism (D) } \\
\hline preoperative & $-0.98 \pm 1.0$ & -3.5 to 0 & $-1.27 \pm 1.2$ & -5.50 to 0 & .275 \\
\hline $3 \mathrm{mos}$ & $-1.38 \pm 1.2$ & -5.0 to 0 & $-4.17 \pm 3.4^{b}$ & -14.0 to 0 & $<.001$ \\
\hline $6 \mathrm{mos}$ & $-1.46 \pm 1.3$ & -5.0 to 0 & $-3.21 \pm 1.9^{b}$ & -8.00 to 0 & $<.001$ \\
\hline \multirow[t]{2}{*}{$12 \mathrm{mos}$} & $-1.22 \pm 1.1$ & -4.0 to 0 & $-2.98 \pm 2.0^{b}$ & -8.75 to 0 & $<.001$ \\
\hline & $P=.358^{c}$ & & $P<.001^{c}$ & & \\
\hline \multicolumn{6}{|c|}{ Topographic astigmatism (D) } \\
\hline preoperative & $1.38 \pm 0.6$ & 0.47 to 2.90 & $2.16 \pm 1.4$ & 0.50 to 6.50 & .577 \\
\hline $3 \mathrm{mos}$ & $1.87 \pm 1.1$ & 0.25 to 4.30 & $4.59 \pm 2.9^{b}$ & 1.00 to 15.70 & $<.001$ \\
\hline $6 \mathrm{mos}$ & $1.72 \pm 1.0$ & 0.27 to 4.14 & $3.74 \pm 1.7^{b}$ & 0.50 to 6.83 & $<.001$ \\
\hline \multirow[t]{2}{*}{$12 \mathrm{mos}$} & $1.58 \pm 1.2$ & 0.27 to 6.40 & $3.67 \pm 1.8^{b}$ & 1.40 to 7.70 & $<.001$ \\
\hline & $P=.469^{c}$ & & $P<.001^{\mathrm{c}}$ & & \\
\hline
\end{tabular}

$\mathrm{D}=$ diopters; FS DSEK = femtosecond laser-assisted Descemet stripping endothelial keratoplasty; mos = months; $\mathrm{PK}=$ penetrating

keratoplasty; $\mathrm{SD}=$ standard deviation.

a $P$ value between FS DSEK and PK.

${ }^{\mathrm{b}} P<.05$ versus preoperative in a linear regression model.

' $P$ value of a linear regression model with 3 postoperative periods at the same time in the model. 


\section{Uncorrected visual acuity and best spectacle-corrected visual acuity}

Before surgery and at 3,6, and 12 months after surgery, there was no significant difference in UCVA between the FS DSEK and PK groups (Table 4). In the FS DSEK group, UCVA was significantly better at all postoperative visits compared with that before surgery. The UCVA in the PK group was significantly improved at 3 and 12 months after surgery compared with that before surgery.

Before surgery, there was no significant difference in BSCVA between the FS DSEK and PK groups ( $0.82 \pm 0.4 \log M A R$ vs $0.73 \pm 0.4 \log M A R$, respectively; $P=.316)$. In both groups, BSCVA showed a significant improvement after surgery. After surgery, the mean BSCVA in the PK group was significantly better when compared with the FS DSEK group at all follow-up visits. The mean gain in BSCVA at 12 months of follow-up was not sig-

TABLE 4. Preoperative and Postoperative Visual Outcome of Femtosecond Laser-Assisted Descemet-Stripping Endothelial Keratoplasty and Penetrating Keratoplasty

\begin{tabular}{|c|c|c|c|c|c|}
\hline & $\begin{array}{c}\text { FS DSEK, Mean } \\
\pm S D\end{array}$ & Range & $\mathrm{PK}$, Mean $\pm \mathrm{SD}$ & Range & $P$ Value $^{\text {a }}$ \\
\hline \multicolumn{6}{|c|}{ UCVA (logMAR) } \\
\hline Preoperative & $1.01 \pm 0.4(20 / 200)$ & 0.22 to 2.0 & $0.88 \pm 0.4(20 / 150)$ & 0.40 to 2.0 & .133 \\
\hline $3 \mathrm{mos}$ & $0.80 \pm 0.2(20 / 125)^{b}$ & 0.40 to 1.70 & $0.71 \pm 0.3(20 / 100)^{b}$ & 0.10 to 1.36 & .144 \\
\hline $6 \mathrm{mos}$ & $0.79 \pm 0.3(20 / 125)^{b}$ & 0.40 to 1.70 & $0.79 \pm 0.3(20 / 125)$ & 0.14 to 1.36 & .959 \\
\hline \multirow[t]{2}{*}{$12 \mathrm{mos}$} & $0.73 \pm 0.3(20 / 102)^{b}$ & 0.34 to 1.70 & $0.68 \pm 0.3(20 / 96)^{b}$ & 0.10 to 1.46 & .539 \\
\hline & $P=.001^{\mathrm{c}}$ & & $P=.045^{c}$ & & \\
\hline \multicolumn{6}{|c|}{ BSCVA (logMAR) } \\
\hline Preoperative & $0.82 \pm 0.4(20 / 132)$ & 0.22 to 2.0 & $0.73 \pm 0.4(20 / 105)$ & 0.18 to 2.0 & .316 \\
\hline $3 \mathrm{mos}$ & $0.65 \pm 0.3(20 / 90)^{b}$ & 0.22 to 1.70 & $0.40 \pm 0.2(20 / 50)^{b}$ & -0.04 to 1.12 & $<.001$ \\
\hline $6 \mathrm{mos}$ & $0.64 \pm 0.3(20 / 87)^{b}$ & 0.26 to 1.70 & $0.35 \pm 0.2(20 / 44)^{b}$ & 0 to 0.86 & $<.001$ \\
\hline \multirow[t]{2}{*}{$12 \mathrm{mos}$} & $0.55 \pm 0.2(20 / 70)^{b}$ & 0.16 to 1.10 & $0.35 \pm 0.2(20 / 44)^{b}$ & -0.04 to 0.98 & $<.001$ \\
\hline & $P=.004^{c}$ & & $P<.001^{c}$ & & \\
\hline \multicolumn{6}{|c|}{ BSCVA gain (logMAR) } \\
\hline $3 \mathrm{mos}$ & $0.15 \pm 0.4$ & -1.06 to 1.34 & $0.33 \pm 0.4$ & -0.48 to 1.48 & .052 \\
\hline $6 \mathrm{mos}$ & $0.14 \pm 0.4$ & -1.06 to 1.30 & $0.38 \pm 0.4$ & -0.40 to 1.46 & .017 \\
\hline $12 \mathrm{mos}$ & $0.24 \pm 0.4$ & -0.34 to 1.50 & $0.38 \pm 0.4$ & -0.24 to 1.30 & .103 \\
\hline
\end{tabular}


nificantly different between the FS DSEK and PK group (0.24 logMAR vs 0.38 logMAR, respectively $P=.103$ ).

\section{Visual symptom score}

Before surgery, the most commonly reported symptom in the FS DSEK and PK groups was blurry vision (Table 5), with a small significant difference between the 2 groups. In the FS DSEK group, the percentage of patients reporting no blurry vision increased from $8.3 \%$ before surgery to $25.9 \%$ at 12 months after surgery. In the PK group, $43.2 \%$ reported no blurry vision at 12 months after surgery as compared with $0 \%$ before surgery. The number of patients reporting double vision or distorted vision was not significantly different between the FS DSEK and PK groups during the 12 months of follow-up.

TABLE 5. Subjective Visual Symptoms after Femtosecond Laser-Assisted Descemet-Stripping Endothelial Keratoplasty and Pentrating Keratoplasty

\begin{tabular}{|c|c|c|c|c|c|c|c|c|c|}
\hline & \multicolumn{4}{|c|}{ FS DSEK } & \multicolumn{4}{|c|}{ PK } & \multirow[b]{2}{*}{$P$ Value } \\
\hline & Preoperative & 3 Mos & 6 Mos & 12 Mos & Preoperative & 3 Mos & 6 Mos & 12 Mos & \\
\hline \multicolumn{10}{|l|}{ Blurry vision (\%) } \\
\hline Great deal & 41.7 & 20.0 & 19.4 & 3.7 & 35.0 & 18.9 & 7.9 & 5.4 & Preoperative, .046 \\
\hline Moderate amount & 36.1 & 20.0 & 12.9 & 11.1 & 27.5 & 8.1 & 15.8 & 21.6 & $3 \mathrm{mos}, .535$ \\
\hline Little & 13.9 & 36.7 & 32.3 & 59.3 & 37.5 & 45.9 & 39.5 & 29.7 & $6 \mathrm{mos}, .558$ \\
\hline None & 8.3 & 23.3 & 35.5 & 25.9 & 0.0 & 27.0 & 36.8 & 43.2 & 12 mos, .131 \\
\hline \multicolumn{10}{|c|}{ Double or distorted vision(\%) } \\
\hline Great deal & 5.6 & 10.0 & 3.2 & 3.8 & 7.5 & 7.9 & 7.9 & 2.7 & Preoperative, .487 \\
\hline Moderate amount & 13.9 & 10.0 & 22.6 & 7.7 & 10.0 & 15.8 & 15.8 & 10.8 & $3 \mathrm{mos}, .424$ \\
\hline Little & 36.1 & 20.0 & 25.8 & 42.3 & 22.5 & 34.2 & 36.8 & 24.3 & $6 \mathrm{mos}, .567$ \\
\hline None & 44.4 & 60.0 & 48.4 & 46.2 & 60.0 & 42.1 & 39.5 & 62.2 & 12 mos, .479 \\
\hline \multicolumn{10}{|l|}{ Glare (\%) } \\
\hline Great deal & 25.0 & 13.3 & 9.7 & 15.4 & 20.0 & 13.2 & 10.5 & 8.1 & Preoperative, .050 \\
\hline Moderate amount & 38.9 & 30.0 & 19.4 & 3.8 & 15.0 & 21.1 & 23.7 & 5.4 & $3 \mathrm{mos}, .812$ \\
\hline Little & 16.7 & 40.0 & 54.8 & 53.8 & 37.5 & 42.1 & 26.3 & 45.9 & $6 \mathrm{mos}, .075$ \\
\hline None & 19.4 & 16.7 & 16.1 & 26.9 & 27.5 & 23.7 & 39.5 & 40.5 & $12 \mathrm{mos}, .613$ \\
\hline \multicolumn{10}{|l|}{ Halo (\%) } \\
\hline Great deal & 19.4 & 6.7 & 9.7 & 3.8 & 23.1 & 15.8 & 5.3 & 8.1 & Preoperative, .241 \\
\hline Moderate amount & 22.2 & 16.7 & 19.4 & 19.2 & 15.4 & 28.9 & 18.4 & 24.3 & $3 \mathrm{mos}, .327$ \\
\hline Little & 25.0 & 33.3 & 29.0 & 46.2 & 43.6 & 23.7 & 31.6 & 29.7 & $6 \mathrm{mos}, .911$ \\
\hline None & 33.3 & 43.3 & 41.9 & 30.8 & 17.9 & 31.6 & 44.7 & 37.8 & $12 \mathrm{mos}, .580$ \\
\hline \multicolumn{10}{|c|}{ Colors look different (\%) } \\
\hline Great deal & 5.6 & 0.0 & 6.5 & 0.0 & 5.0 & 0.0 & 2.6 & 2.7 & Preoperative, .428 \\
\hline Moderate amount & 2.8 & 13.8 & 3.2 & 3.8 & 7.5 & 5.3 & 0.0 & 0.0 & $3 \mathrm{mos}, .321$ \\
\hline Little & 22.2 & 10.3 & 9.7 & 15.4 & 10.0 & 5.3 & 13.2 & 13.5 & 6 mos. .572 \\
\hline None & 69.4 & 75.9 & 80.6 & 80.0 & 77.5 & 89.5 & 84.2 & 83.8 & $12 \mathrm{mos}, .536$ \\
\hline
\end{tabular}

FS DSEK = femtosecond laser-assisted Descemet stripping endothelial keratoplasty; Mos = months; PK = penetrating keratoplasty. 
In both the FS DSEK and PK groups, a relatively high percentage of patients reported glare and halo symptoms before surgery (glare, $80.6 \%$ vs $72.5 \%$, respectively; halo, $66.7 \%$ vs $82.1 \%$, respectively). During 12 months of follow-up, the percentage of patients reporting glare symptoms (severity score 3 ) decreased from $25.0 \%$ to $15.4 \%$ in the FS DSEK group and from $20.0 \%$ to $8.1 \%$ in the PK group (Table 5). The percentage of patients reporting halos decreased from $19.4 \%$ before surgery to $3.8 \%$ at 12 months after surgery in the FS DSEK group and from $23.1 \%$ before surgery to $8.1 \%$ at 12 months after surgery in the PK group (Table 5).

Before surgery, there was no significant difference in visual symptom score between the FS DSEK and PK groups ( $6.33 \pm 3.0$ and $5.70 \pm 2.7 ; P=.343)$. During follow-up, the difference in visual symptom score between the FS DSEK and PK groups was not significant (3 months, $4.70 \pm 3.5$ vs $4.74 \pm 3.2[P=.964] ; 6$ months, $4.52 \pm 3.7$ vs $3.97 \pm 2.7[P=.484]$; 12 months, $3.78 \pm 2.8$ vs $3.49 \pm 2.9[P=.694])$. In both groups, there was a significant improvement of visual symptom score at all time points (FS DSEK, $P=.017 ; \mathrm{PK}, P=.006$ ).

\section{DISCUSSION}

The purpose of this randomized, multicenter trial was to evaluate the quality of vision (intraocular straylight and contrast sensitivity) and to correlate these quality-ofvision parameters to the refractive and visual outcomes after FS DSEK and PK. Previous studies reported limited visual outcomes after lamellar keratoplasty because of an interface haze, which may increase straylight. ${ }^{13,27}$ The main conclusion of our study is that both FS DSEK and PK are very effective at improving straylight, and no significant difference between the 2 groups was found. Although postoperative straylight is still increased, the increase is only 2 -fold when compared with age-matched controls. In this group of patients with severely impaired vision, the reliability parameter (expected standard deviation) for straylight measurements proved to be very effective to ensure accurate measurements before and after surgery (Figure 2). This compared well with published values between 0.08 and 0.06 as well as with the limit values used for expected standard deviation of $0.08 .^{19}$

The straylight values in our study showed a significant improvement during the 12 months of follow-up in the FS DSEK and PK group. The largest improvement was seen from baseline to 3 months after surgery, and after that, straylight values remained stable up to 12 months of follow-up. A previous randomized clinical trial comparing deep lamellar endothelial keratoplasty (DLEK) with PK showed no significant change in straylight values after surgery. ${ }^{12}$ This difference in outcome may be explained by several factors. First, the preoperative straylight value reported in our study obviously was higher than in the study by Patel and associates, which may be the result of a higher degree of 
corneal edema resulting from long-term corneal decompensation. ${ }^{12}$ Furthermore, the preoperative BSCVA was better in the study by Patel and associates, which also may indicate a lower degree of corneal edema or a shorter duration of corneal decompensation. $^{12}$

After surgery, the percentage of patients with straylight values within the normal agematched range increased from $13.9 \%$ to $38.5 \%$ in the FS DSEK group and from $12.5 \%$ to $45.7 \%$ in the PK group. Despite the FS DSEK and PK procedure, 50.0\% of the FS DSEK patients and $51.4 \%$ of the PK patients did not return to a normal straylight level. In contrast to the previously reported correlation between age and straylight after DSEK, we did not find a significant correlation between age and straylight after FS DSEK and PK. ${ }^{28}$ Postoperative straylight values were compared with those of normal age-matched controls, but the age of the donor cornea was not age-matched with the recipient cornea. This also may explain why the postoperative straylight values of the study eyes did not return to a normal straylight level. Also, 6 of 36 eyes in the FS DSEK group had residual central corneal haze after deturgescence of the recipient cornea. However, because the group of patients with a central corneal haze was small, we only can speculate whether this may influence the postoperative straylight value.

It has been suggested that BSCVA after endothelial keratoplasty is limited because of increased straylight values associated with the lamellar interface. ${ }^{12,13}$ In our study, the gain of BSCVA and straylight values was not statistically significant between the 2 groups. The correlation between the change in straylight and BSCVA from baseline to 12 months after surgery was significantly higher in the FS DSEK compared with the PK group, which was comparable with the results of a previous study. ${ }^{12}$ It also was reported that the greatest improvement of BSCVA after PK and DLEK occurred in the first 3 months after surgery, which was comparable with our speed of improvement of UCVA and BSCVA in the FS DSEK and PK group.

Contrast sensitivity can be affected by corneal edema, distortion, or cataract. In our study, we found a significant improvement of contrast sensitivity after FS DSEK and PK. There was no significant difference in contrast sensitivity between the FS DSEK and PK groups during the 12 months of follow-up. The contrast sensitivity test was performed with the best spectacle correction in place, which corrected the higher astigmatism in the PK group. The contrast sensitivity reported in our study is comparable with that of previous randomized studies comparing deep anterior lamellar keratoplasty or DLEK and PK and lower than the contrast sensitivity of healthy eyes. ${ }^{12,29,30}$

Improvement of BSCVA correlated with an increase in contrast sensitivity after FS DSEK and PK. The lamellar interface prepared with the FS laser showed no significant decrease in contrast sensitivity when compared with the PK group. Furthermore, the largest improvement in contrast sensitivity was seen in the first 3 months after surgery, which was comparable with our other outcomes (straylight, UCVA, and BSCVA). When 
evaluating the binocular contrast sensitivity, there was a significant improvement in the PK group, but not in the FS DSEK group. This may be explained by the difference of the contrast sensitivity between the study eye and fellow eye within the 2 study groups. The difference between the study eye and the fellow eye in the PK group was larger as compared with that of the FS DSEK group. It can be speculated that a fellow eye in the PK group with a higher contrast sensitivity will provide a better binocular contrast sensitivity.

The BSCVA in our study was significantly better in the PK group compared with the FS DSEK group. This is in comparison with earlier studies and may be explained by a possible interface haze or irregularities of the interface. ${ }^{1,31}$ However, a randomized study showed a comparable BSCVA in DLEK and PK eyes during the 1-year follow-up. ${ }^{12}$ Recent DSAEK studies have reported a mean BSCVA of 20/34 to 20/44 at 6 to 12 months after surgery, respectively, ${ }^{6,31,32}$ which is higher than our results for the FS DSEK group. This may be explained by an increased opacification at the interface as a result of keratocyte activation by the FS laser or of a suboptimal smoothness of the stromal bed as compared with a microkeratome-prepared bed. ${ }^{33,34}$ Further, as previously mentioned, there were 6 patients in the FS DSEK group with residual central haze after deturgescence of the recipient cornea, and this may influence the final BSCVA.

Before surgery, blurry vision was the most frequently reported symptom in both the FS DSEK and PK groups, which is comparable with the results of the study by Boisjoly and associates. ${ }^{26}$ Twelve months after surgery, the percentage of patients with blurry vision decreased from $91.7 \%$ to $74.1 \%$ in the FS DSEK group and from $100 \%$ to $56.8 \%$ in the PK group. The preoperative visual symptom scores of our FS DSEK and PK groups were slightly higher when compared with those of a previous study. ${ }^{26}$ This may be explained by the difference of the different diagnosis of the graft candidates between the 2 studies.

Although astigmatism was significantly higher in the PK group, the percentage of patients with symptoms of double vision or distorted vision was comparable between the FS DSEK and PK groups. Patients were instructed to answer the questions on visual symptoms when using their spectacle or contact lens correction, which could have influenced their response.

In the PK group, there were 7 patients who were unable to wear a correction because of anisometropia, in contrast to no patients in the FS DSEK group. The anisometropia can be explained by the higher amount of astigmatism in the PK group. This illustrates a clear advantage of FS DSEK in comparison with PK, which is a lower and stable astigmatism, as has been described previously. ${ }^{11}$

In conclusion, this randomized study showed that FS DSEK resulted in an equally good improvement of straylight and contrast sensitivity when compared with PK. In addition, corneal astigmatism did not increase after FS DSEK. However, although the UCVA in 
both groups was comparable and the visual symptom score decreased in both groups, BSCVA was slightly better in the PK group. Our results indicate that the quality of vision measured by contrast sensitivity, straylight, and changes in visual acuity after FS DSEK is comparable with that achieved after PK. 


\section{REFERENCES}

1. Williams KA, Muehlberg SM, Lewis RF, Coster DJ. How successful is corneal transplantation? A report from the Australian Corneal Graft Register. Eye (Lond) 1995;9(Pt 2):219-227.

2. Williams KA, Esterman AJ, Bartlett C, Holland H, Hornsby NB, Coster DJ. How effective is penetrating corneal transplantation? Factors influencing long-term outcome in multivariate analysis. Transplantation 2006;81(6):896-901.

3. Tahzib NG, Cheng YY, Nuijts RM. Three-year follow-up analysis of Artisan toric lens implantation for correction of postkeratoplasty ametropia in phakic and pseudophakic eyes. Ophthalmology 2006;113(6):976-984.

4. Tillett CW. Posterior lamellar keratoplasty. Am J Ophthalmol 1956;41(3):530 -533.

5. Cheng YY, Pels E, Nuijts RM. Femtosecond-laser-assisted Descemet's stripping endothelial keratoplasty. J Cataract Refract Surg 2007;33(1):152-155.

6. Gorovoy MS. Descemet-stripping automated endothelial keratoplasty. Cornea 2006;25(8):886-889.

7. Melles GR, Eggink FA, Lander F, et al. A surgical technique for posterior lamellar keratoplasty. Cornea 1998;17(6):618-626.

8. Price FW Jr, Price MO. Descemet's stripping with endothelial keratoplasty in 200 eyes: early challenges and techniques to enhance donor adherence. J Cataract Refract Surg 2006; 32(3):411-418.

9. Terry MA, Ousley PJ. Deep lamellar endothelial keratoplasty in the first United States patients: early clinical results. Cornea 2001;20(3):239-243.

10. Terry MA. Endothelial keratoplasty: history, current state, and future directions. Cornea 2006;25(8):873-878.

11. Cheng YY, Schouten JS, Tahzib NG, et al. Efficacy and safety of femtosecond laser-assisted corneal endothelial keratoplasty: a randomized multicenter clinical trial. Transplantation 2009;88(11):1294-1302.

12. Patel SV, McLaren JW, Hodge DO, Baratz KH. Scattered light and visual function in a randomized trial of deep lamellar endothelial keratoplasty and penetrating keratoplasty. Am J Ophthalmol 2008;145(1):97-105.

13. Rich LF. Expanding the scope of lamellar keratoplasty. Trans Am Ophthalmol Soc 1999;97:771-814.

14. Musch DC, Farjo AA, Meyer RF, Waldo MN, Janz NK. Assessment of health-related quality of life after corneal transplantation. Am J Ophthalmol 1997;124(1):1-8.

15. Rubin GS, Bandeen-Roche K, Huang GH, et al. The association of multiple visual impairments with self-reported visual disability: SEE project. Invest Ophthalmol Vis Sci 2001;42(1):64-72.

16. West SK, Rubin GS, Broman AT, Munoz B, Bandeen-Roche K, Turano K. How does visual impairment affect performance on tasks of everyday life? The SEE Project. Salisbury Eye Evaluation. Arch Ophthalmol 2002;120(6):774-780.

17. Williams KA, Ash JK, Pararajasegaram P, Harris S, Coster DJ. Long-term outcome after corneal transplantation. Visual result and patient perception of success. Ophthalmology 1991;98(5):651-657.

18. Franssen L, Coppens JE, van den Berg TJ. Compensation comparison method for assessment of retinal straylight. Invest Ophthalmol Vis Sci 2006;47(2):768-776.

19. van den Berg TJ, van Rijn LJ, Michael R, et al. Straylight effects with aging and lens extraction. Am J Ophthalmol 2007;144(3):358-363.

20. Elliott DB, Sanderson K, Conkey A. The reliability of the Pelli-Robson contrast sensitivity chart. Ophthalmic Physiol Opt 1990;10(1):21-24.

21. Elliott DB, Bullimore MA. Assessing the reliability, discriminative ability, and validity of disability glare tests. Invest Ophthalmol Vis Sci 1993;34(1):108-119. 
22. Pesudovs K, Hazel CA, Doran RM, Elliott DB. The usefulness of Vistech and FACT contrast sensitivity charts for cataract and refractive surgery outcomes research. Br J Ophthalmol 2004;88(1):11-16.

23. Pelli DG, Robson JG, Wilkens AJ. The design of a new letter chart of measuring contrast sensitivity. Clinical Vision Science 1988;2(3):187-199.

24. Ferris FL 3rd, Kassoff A, Bresnick GH, Bailey I. New visual acuity charts for clinical research. Am J Ophthalmol 1982; 94(1):91-96.

25. Steinberg EP, Tielsch JM, Schein OD, et al. The VF-14. An index of functional impairment in patients with cataract. Arch Ophthalmol 1994;112(5):630-638.

26. Boisjoly $\mathrm{H}$, Gresset J, Fontaine $\mathrm{N}$, et al. The VF-14 index of functional visual impairment in candidates for a corneal graft. Am J Ophthalmol 1999;128(1):38-44.

27. Soong HK, Mian S, Abbasi O, Juhasz T. Femtosecond laser-assisted posterior lamellar keratoplasty: initial studies of surgical technique in eye bank eyes. Ophthalmology 2005;112(1):44-49.

28. Patel SV, Baratz KH, Hodge DO, Maguire LJ, McLaren JW. The effect of corneal light scatter on vision after Descemet stripping with endothelial keratoplasty. Arch Ophthalmol 2009;127(2):153-160.

29. Mantyjarvi M, Laitinen T. Normal values for the Pelli-Robson contrast sensitivity test. J Cataract Refract Surg 2001;27(2):261-266.

30. Shimazaki J, Shimmura S, Ishioka M, Tsubota K. Randomized clinical trial of deep lamellar keratoplasty vs penetrating keratoplasty. Am J Ophthalmol 2002;134(2):159-165.

31. Bahar I, Kaiserman I, McAllum P, Slomovic A, Rootman D. Comparison of posterior lamellar keratoplasty techniques to penetrating keratoplasty. Ophthalmology 2008;115(9):1525-1533.

32. Covert DJ, Koenig SB. New triple procedure: Descemet's stripping and automated endothelial keratoplasty combined with phacoemulsification and intraocular lens implantation. Ophthalmology 2007;114(7):1272-1277.

33. Hindman HB, McCally RL, Myrowitz E, et al. Evaluation of deep lamellar endothelial keratoplasty surgery using scatterometry and wavefront analyses. Ophthalmology 2007;114(11):2006-2012.

34. Jones YJ, Goins KM, Sutphin JE, Mullins R, Skeie JM. Comparison of the femtosecond laser (IntraLase) versus manual microkeratome (Moria ALTK) in dissection of the donor in endothelial keratoplasty: initial study in eye bank eyes. Cornea 2008;27(1):88-93. 



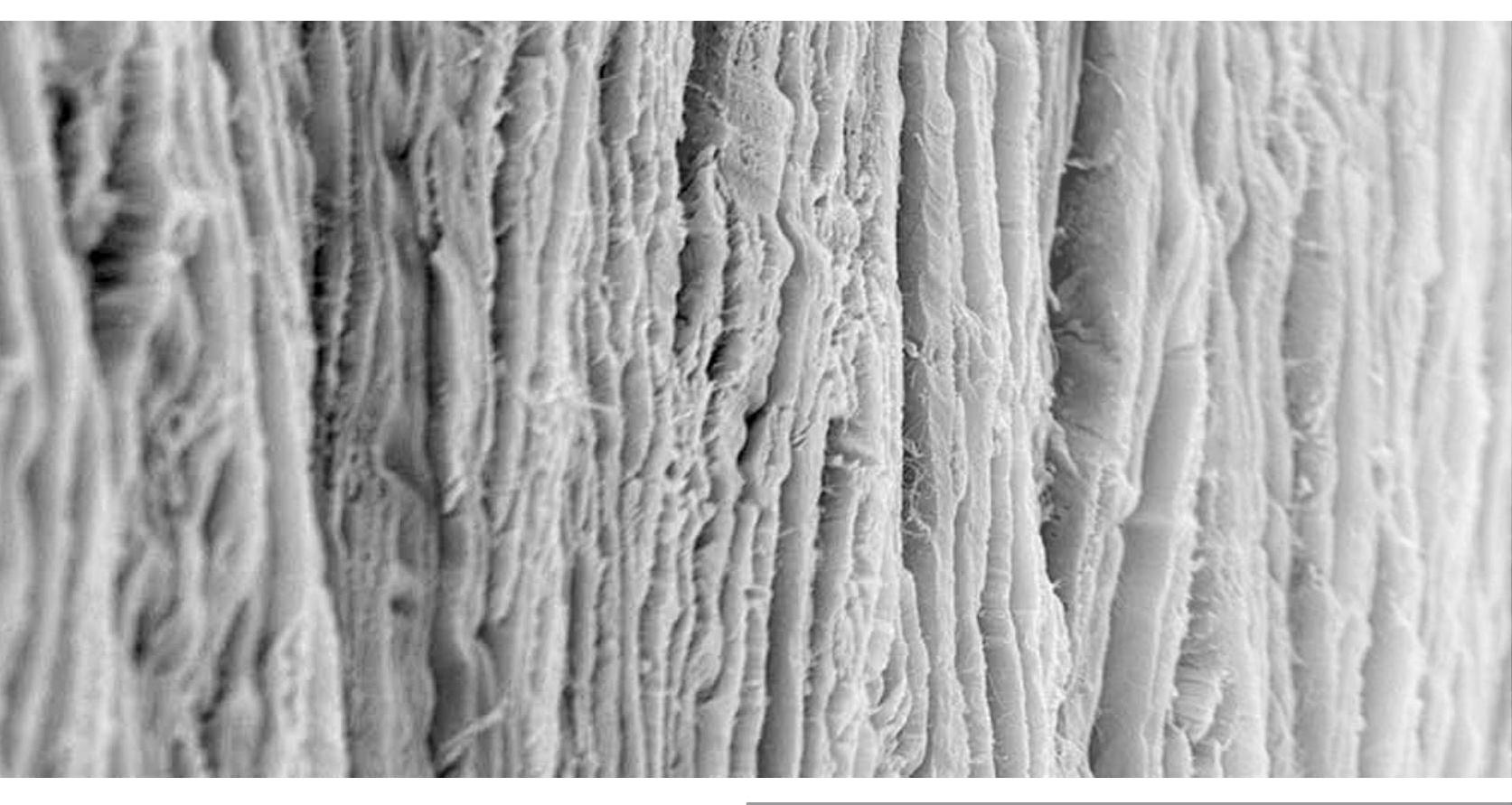




\title{
CHAPTER 8
}

Economic Evaluation of Endothelial Keratoplasty Techniques and Penetrating Keratoplasty in The Netherlands

\author{
Frank J.H.M. van den Biggelaar \\ Yanny Y.Y. Cheng \\ Rudy M.M.A. Nuijts \\ Jan S.A.G. Schouten \\ Robert-Jan Wijd \\ Elisabeth Pels \\ Hugo van Cleynenbreugel \\ Catharina A. Eggink \\ Wilhelmina J. Rijneveld \\ Carmen D. Dirksen
}

Am J Ophthalmol. 2012 Aug;154(2):272-281 


\section{ABSTRACT}

Purpose: To evaluate cost-effectiveness of penetrating keratoplasty (PK), femtosecond Descemet stripping endothelial keratoplasty (FS-DSEK) and Descemet stripping automated endothelial keratoplasty (DSAEK).

Design: Cost-effectiveness analysis based on data from a randomized multicenter clinical trial and a noncomparative prospective study.

Methods: Data of 118 patients with corneal endothelial dysfunction were analyzed in the economic evaluation. Forty patients were included in the PK group, 36 in the FSDSEK group, and 42 in the DSAEK group. The primary incremental cost-effectiveness ratio (ICER) was the incremental costs per clinically improved patient, defined as a patient with a combined effectiveness of both a clinically improved BSCVA (defined as an improvement of at least 2 lines) and a clinically acceptable refractive astigmatism (defined as less than or equal to 3.0 diopters). Analysis was based on a 1-year follow-up period after transplantation.

Results: The percentage of treated patients who met the combined effectiveness measures was $52 \%$ for DSAEK, $44 \%$ for PK and $43 \%$ for FS-DSEK. Mean total costs per patient were $€ 6674$ (US\$ 7942), $€ 12443$ (US\$ 14807) and $€ 7072$ (US\$ 8416) in the PK group, FS-DSEK group and DSAEK group, respectively. FS-DSEK was less effective and more costly compared to both DSAEK and PK. DSAEK was more costly but also more effective compared to PK, resulting in incremental costs of $€ 4975$ (US\$5920) per additional clinically improved patient.

Conclusions: The results of this study show that FS-DSEK was not cost-effective compared to PK and DSAEK. DSAEK, on the other hand, was more costly but also more effective compared to PK. Including societal costs, a longer follow-up period and preparation of the lamellar transplant buttons in a national cornea bank could improve the costeffectiveness of DSAEK. 


\section{INTRODUCTION}

For many years, penetrating keratoplasty (PK) has been the gold-standard technique for corneal transplantation resulting from endothelial disease or endothelial dysfunction. Several studies have shown that the technique is safe and effective. ${ }^{1,2}$ However, PK has several drawbacks, including slow visual recovery, suture-related events, graft failure, and wound healing problems. ${ }^{3}$

In recent years, interest has grown in endothelial keratoplasty (EK), in which only the diseased endothelium is transplanted and the healthy anterior stromal tissue is preserved. As compared to PK, EK shows faster visual recovery, less change in astigmatism, better stability and predictability of the postoperative refraction, and fewer suture-related problems. ${ }^{4-6}$ However, several EK studies found an increased risk of graft dislocation and higher endothelial cell loss. ${ }^{4,7-10}$

In EK, different techniques have been developed for the preparation of the donor cornea, including Descemet stripping automated endothelial keratoplasty (DSAEK), where the donor cornea is prepared with a microkeratome, ${ }^{11,12}$ and femtosecond laserassisted Descemet stripping endothelial keratoplasty (FS-DSEK), where the donor tissue is prepared with a femtosecond laser. ${ }^{13-15}$

The efficacy and visual outcomes of EK techniques vs PK have been well studied. ${ }^{16-18}$ However, the efficiency of the techniques is less elucidated. In an economic evaluation, outcomes and costs of interventions are compared ${ }^{19}$ with the aim to improve resource allocation decisions by policymakers and insurers. One type of economic evaluation is the cost-effectiveness analysis, in which the costs of an intervention are related to a single outcome measure, for example "clinical success."

In this study, we performed an evaluation of the cost-effectiveness of FS-DSEK, DSAEK, and $\mathrm{PK}$, for which data from a randomized multicenter clinical trial and a noncomparative prospective study were used.

\section{PATIENTS AND METHODS}

\section{Study population}

In this study, costs and effects of PK and 2 different techniques of EK (ie, FS-DSEK and DSAEK) were compared. Empirical data were obtained from 2 sources. First, PK and FS-DSEK were compared in a randomized clinical trial, the Dutch Lamellar Corneal Transplantation Study (DLCTS), which was performed between April 2005 and April 2007. Patients were included in 5 ophthalmic centers in The Netherlands. The study was approved by the institutional review boards of all participating centers. Before inclusion, 
informed consent was obtained from all patients. More details about the safety, efficacy and quality of vision of PK vs FS-DSEK are described elsewhere. ${ }^{16,20}$

Second, a third group of patients who received a DSAEK transplantation in Maastricht University Medical Center between April 17, 2008 and March 11, 2010 was included in the study. Inclusion in the economic evaluation was based on the completion of qualityof-life questionnaires on at least 2 out of 3 measurements.

For all 3 groups, inclusion criteria were patients with Fuchs endothelial dystrophy, aphakic or pseudophakic bullous keratopathy (ABK/PBK), posterior polymorphous dystrophy, or iridocorneal endothelial syndrome; a minimum patient age of 18 years; and best spectacle-corrected visual acuity (BSCVA) lower than 20/50. Patients with mental retardation, previous $\mathrm{PK}$, and human leukocyte antigen-typed keratoplasty were excluded.

All patients underwent a comprehensive ophthalmic examination and completed quality-of-life questionnaires. Data were obtained at study inclusion (T0) and 6 (T1) and 12 (T2) months after the transplantation, which was 9 and 15 months after baseline measurement, respectively.

\section{Surgical procedures}

In the FS-DSEK group, all donor posterior lamellar discs (PLDs) were prepared in Maastricht University Medical Center (MUMC) with a 30-kHz femtosecond laser (AMOIntralase Corp, Irvine, California, USA), as previously described. ${ }^{15}$ In the DSAEK group, the PLDs were created with the use of a microkeratome (Moria Inc, Doylestown, Pennsylvania, USA). In both groups, an 8.0-mm donor corneal disc was trephined from the corneoscleral button with a disposable Barron trephine. In the recipient, a 5.0- $\mathrm{mm}$ corneoscleral incision and 2 limbal paracenteses were made. The Descemet membrane was scored and a circle of $7.5 \mathrm{~mm}$ Descemet membrane and endothelium was stripped from the posterior stroma. Four transcorneal incisions were made in the midperipheral recipient cornea to drain fluid between the recipient cornea and PLD. After coating the endothelial surface with viscoelastic material, the PLD was gently folded into a taco configuration and inserted. The corneoscleral incision was closed with 4 10-0 nylon sutures. An air bubble was injected to unfold the PLD and to press the PLD against the recipient cornea.

In the PK group, the recipient cornea was trephined using a $7.75-\mathrm{mm}$ or $8.0-\mathrm{mm}$ Hessburg-Barron vacuum trephine (Barron Precision Instruments, Grand Blanc, Michigan, USA), whereas the donor cornea was trephined with an $8.0-\mathrm{mm}$ or $8.25-\mathrm{mm}$ disposable trephine (Medical Workshop, De Meern, The Netherlands). In all cases, a combined suturing technique of a running 11-0 nylon suture with 8 interrupted 10-0 nylon sutures was performed. Selective suture removal was based on topographic astigmatism pattern. 
In all treatment groups, patients with cataract either underwent primary cataract extraction with posterior chamber intraocular lens $(\mathrm{IOL})$ implantation either before the corneal transplantation or combined with EK or PK in 1 surgical session.

\section{Economic evaluation}

The economic evaluation was performed from a health care perspective with a time horizon of 15 months, from study inclusion (about 3months before surgery) up to 12 months after surgery. All relevant resources consumed within the health care sector were taken into account.

Effectiveness was based on 3 outcome measures. Two clinical outcome measures were used, namely the BSCVA and the refractive astigmatism. Furthermore, the National Eye Institute Visual Functioning Questionnaire (NEI VFQ-25) was used as an outcome measure, reflecting vision-specific health-related quality of life.

To evaluate cost-effectiveness, incremental cost-effectiveness ratios (ICERs) were calculated by dividing the difference in costs by the difference in effectiveness between 2 treatments. The primary ICER was the incremental costs per clinically improved patient, defined as a patient with a combined effectiveness of both a clinically improved BSCVA and a clinically acceptable refractive astigmatism. In the secondary analyses, other outcome measures were used, namely costs per improved patient on the NEI VFQ-25 and costs per improved patient on all 3 outcome measures.

\section{Best spectacle-corrected visual acuity}

The BSCVA was determined using the Early Treatment of Diabetic Retinopathy Study (ETDRS) letter charts and was converted to logarithm of the minimal angle of resolution (logMAR) measurements. ${ }^{21}$ Vision levels of counting fingers, hand movements, light perception, and no light perception were substituted by logMAR values of 1.7, 2.0, 2.5, and 3.0, respectively. A patient with an improvement of at least 2 lines on the ETDRS letter chart between T0 and T2 was considered to be clinically improved. 22,23

\section{Refractive astigmatism}

Refractive astigmatism was used as an outcome measure because a lower level of postoperative astigmatism is a major advantage of EK techniques compared to PK and refractive astigmatism is considered to be more important for the patient compared to topographic astigmatism. ${ }^{24}$ In this study, a postoperative refractive astigmatism less than or equal to 3.0 diopters (D) at T2 was considered to be clinically acceptable. ${ }^{16}$

National eye institute visual functioning questionnaire

The NEI VFQ-25 is a vision-specific health-related quality-of-life questionnaire that measures domains related to daily visual functioning that are most important for patients 
with vision deficits. The NEI VFQ- 25 consists of 25 questions that can be expanded with 13 additional items. All questions comprise 12 subscales. Eleven of them can eventually be converted into 1 single score. The remaining subscale (general health) is included in the questionnaire to provide robust information about an individual's general health status. After recoding, the scores of the subscales and the single composite score range from 0 (worst possible value) to 100 (best possible value). As several studies have suggested that a 10-point change in the composite score is clinically important, ${ }^{25-27}$ a patient with a minimum gain of 10 points in the composite score at T2 as compared to T0 was considered to be clinically improved in this study.

\section{Cost analysis}

All relevant health care costs of the 3 interventions were assessed according to the Dutch guidelines for cost calculations. ${ }^{28}$ Costs outside the health care sector, such as productivity losses, were excluded from analyses. Costs were calculated by multiplying the volumes of resource use by the cost price per resource unit. All costs were converted to 2010 Euros. Costs occurring after 12 months were discounted at an annual rate of $4 \%$. All costs are reported in Euros $(€)$ and United States dollars (US\$) $\left(€ 1=\right.$ US\$1.19). ${ }^{29}$

Resources included preparation, preservation, allocation, and transportation of the donor corneas, which were provided by the Bio Implant Services (BISLIFE) Foundation (Leiden, The Netherlands); outpatient visits; diagnostic procedures; preparation of the donor PLD; surgical procedures; hospitalization; and postoperative drugs use. Data of outpatient visits, surgical and diagnostic procedures, and hospitalization were obtained from the registries of the participating hospitals.

Services of the BISLIFE Foundation were valued using Dutch reimbursement rates, as no unit prices were available. Costs of outpatient visits, surgical procedures, hospitalization hospitalization, and diagnostic procedures were valued using standardized integral unit prices (consisting of personnel, material, capacity, and overhead costs) as calculated by MUMC, in which $71 \%$ of the patients were included. Integral cost prices of surgical procedures depended on the operation time per patient and were based on 2 cost drivers, namely general operating room costs and specific ophthalmology costs. Costs of preparation of the donor PLD were based on the costs of the femtosecond laser and microkeratome, costs of disposables, and personnel costs. Costs of postoperative drugs were obtained from the Dutch Pharmacotherapeutic Compass. ${ }^{30}$

\section{Statistical analysis}

Outcomes were analyzed according to the intention-to-treat principle. Incomplete data from the NEI VFQ-25, incomplete cost data, and missing data concerning the BSCVA and refractive astigmatism were imputed, using SPSS Multiple Imputation (version 17.0 for Windows, SPSS Inc, Chicago, Illinois, USA), under the assumption that the data were 
missing at random. A linear regression model was used with a total run length of 100 iterations. Covariates included in the imputation model were visual outcome measures, which were obtained at T0, T1, and T2. Furthermore, age, sex, study group, and results on additional questions to measure patient satisfaction after a corneal transplantation were included in the model. In total, 5 imputed data sets were obtained. Data analyses were performed with each of these sets and the results were pooled.

For continuous data, the differences between groups were analyzed using 1-way ANOVA or a linear regression model with 2 dummy variables representing the groups with PK as the reference. The Pearson chi-square test was used to compare categorical data. To analyze the change between the postoperative measurements and the preoperative measurement, a paired sample t test was used. A $P$ value of less than .05 was considered to be statistically significant.

As cost data regularly are highly skewed, traditional parametric and nonparametric statistical methods are not appropriate to analyze the difference in mean costs between groups. ${ }^{31}$ Therefore, we performed nonparametric bootstrapping with 1000 replications to estimate the uncertainty in the incremental costs and effects, using Microsoft Excel for Windows (Microsoft Corp, Redmond, Washington, USA).

To investigate cost-effectiveness, ICERs were calculated. For this purpose, the treatments were ranked from the most effective to the least effective. A treatment that was less effective and more costly than at least 1 alternative was said to be dominated and ruled out from the calculation of ICERs. Then, each treatment was compared to the next most effective treatment by calculating the ICER. Each treatment with a higher ICER than that of a more effective intervention was ruled out based on so-called extended dominance. $^{32,33}$

In order to show the probability of each treatment being the optimal choice for a range of ceiling ratios representing the maximum amount of money that the decision maker is willing to pay for an additional health effect, cost-effectiveness acceptability curves (CEACs) were created. ${ }^{34}$ Based on these curves, the cost-effectiveness frontier can be determined, which indicates which strategy is to be preferred for a range of ceiling ratios. $^{35}$

\section{Sensitivity analyses}

Sensitivity analyses were performed to test the robustness of the results. First, the cutoff point for a patient with an improved BSCVA was at least 2 lines on the ETDRS letter chart between $\mathrm{T} 0$ and $\mathrm{T} 2$. In the sensitivity analyses, we tested the impact on the primary analysis of changing this to at least 1 line and at least 3 lines.

Second, postoperative refractive astigmatism less than or equal to $3.0 \mathrm{D}$ at T2 was considered to be clinically acceptable. In the sensitivity analyses, we tested the impact 
on the primary analysis of changing this to less than or equal to $2.0 \mathrm{D}$ and less than or equal to $4.0 \mathrm{D}$.

Third, it has been stated that a 5-point change in the composite score of the NEI VFQ25 is clinically relevant. ${ }^{25,27}$ Therefore, we considered a patient with a gain of 5 points in the composite score at 12 months postoperatively to be clinically improved.

Fourth, we replaced the cost prices as determined in the MUMC by standardized Dutch unit prices-if available-determined by Oostenbrink and associates. ${ }^{28}$

Finally, the cost price of the femtosecond laser was based on the actual use at the time of the study. Currently, however, the laser is also used for the implantation of intracorneal ring segments in about 30 keratoconus patients per year and in the future will be used for about 300 LASIK procedures per year.

\section{Subgroup analysis}

In all treatment groups, a number of patients with cataract underwent primary cataract extraction with posterior chamber IOL implantation combined with EK or PK in 1 surgical session. As this resulted in an increased operation time for these patients, this could bias the costs per patient. In addition, these patients may have a lower preoperative BSCVA compared to patients who do not have cataract. Therefore, we performed a subgroup analysis on the patients who had only EK or PK surgery without cataract surgery.

\section{RESULTS}

\section{Study population}

Data of 118 patients were analyzed in the economic evaluation. Forty patients were included in the PK group, 36 in the FS-DSEK group, and 42 in the DSAEK group.

In Table 1, baseline patient characteristics of the 3 treatment groups are shown. The mean age was $71.4 \pm 11.3,68.9 \pm 8.8$, and $70.8 \pm 11$ years in the PK group, FS-DSEK group, and DSAEK group, respectively $(P=.59)$. In all groups, the main reason for keratoplasty was Fuchs endothelial dystrophy and pseudophakic bullous keratopathy. In the DSAEK group, more patients were pseudophakic $(27 / 42 ; 64.3 \%)$ as compared to the PK patients $(18 / 40 ; 45.0 \%)$ and FS-DSEK patients $(17 / 36 ; 47.2 \%)$, but this was not significantly different $(P=.08)$.

At 12 months follow-up, only 1 eye in the PK group had all sutures out, whereas the remaining 39 eyes still had most or all of their sutures in.

\section{Effectiveness}

In Table 2, preoperative and postoperative BSCVA and refractive astigmatism are displayed. Preoperatively, mean logMAR BSCVA was $0.72,0.82$, and 0.76 in the PK group, 
TABLE 1. Baseline Characteristics of Study Population $(n=118)$

\begin{tabular}{lcccc}
\hline & PK & FS-DSEK & DSAEK & P value \\
\hline Eyes (n) & 40 & 36 & 42 & NA \\
Age in years (mean + SD) & $71.4 \pm 11.3$ & $68.9 \pm 8.8$ & $70.8 \pm 11$ & $0.59^{\mathrm{b}}$ \\
Women, $\mathbf{n}(\%)$ & $27(67.5 \%)$ & $21(58.3 \%)$ & $27(64.3 \%)$ & $0.70^{\mathrm{c}}$ \\
Diagnosis, $\mathbf{n}(\%)$ & & & $0.66^{\mathrm{c}}$ \\
Fuchs endothelial dystrophy & $20(50 \%)$ & $21(58.3 \%)$ & $22(52.4 \%)$ & \\
Pseudophakic bullous keratopathy & $19(47.5 \%)$ & $15(41.7 \%)$ & $19(45.2 \%)$ & $0.08^{\mathrm{c}}$ \\
Posterior polymorphous dystrophy & $1(2.5 \%)$ & $0(0 \%)$ & $0(0 \%)$ & $1(2.4 \%)$ \\
Iridocorneal endothelial syndrome & $0(0 \%)$ & $0(0 \%)$ & & \\
Recipient lens status ,n (\%) & & & & \\
Aphakic & $1(2.5 \%)^{\mathrm{a}}$ & $0(0 \%)$ & $3(7.1 \%)^{\mathrm{a}}$ & \\
Phakic & $21(52.5 \%)$ & $19(52.8 \%)$ & $12(28.6 \%)$ \\
Pseudophakic & $18(45 \%)$ & $17(47.2 \%)$ & $27(64.3 \%)$ & \\
\hline
\end{tabular}

DSAEK = Descemet stripping automated endothelial keratoplasty; FS-DSEK = femtosecond laser-assisted Descemet stripping endothelial keratoplasty; $\mathrm{NA}=$ not applicable; $\mathrm{PK}=$ penetrating keratoplasty; $\mathrm{SD}=$ standard deviation.

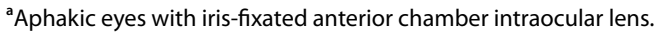

${ }^{\mathrm{b}} 1$-way ANOVA.

'chi-square test.

TABLE.2 Preoperative and Postoperative Best Spectacle-Corrected Visual Acuity and Refractive Astigmatism (Pooled Data) for the 3 Treatment Groups

\begin{tabular}{|c|c|c|c|}
\hline & PK & FS-DSEK & DSAEK \\
\hline \multicolumn{4}{|l|}{ BSCVA (logMAR), mean \pm SD } \\
\hline preoperative & $0.72 \pm 0.38$ & $0.82 \pm 0.42$ & $0.76 \pm 0.45$ \\
\hline 6 months & $0.35 \pm 0.25^{\mathrm{a}}$ & $0.62 \pm 0.30^{\mathrm{a}, \mathrm{b}}$ & $0.34 \pm 0.26^{\mathrm{a}}$ \\
\hline 12 months & $0.36 \pm 0.25^{\mathrm{a}}$ & $0.50 \pm 0.18^{a, b}$ & $0.31 \pm 0.19^{\mathrm{a}}$ \\
\hline \multicolumn{4}{|c|}{ BSCVA gain (logMAR), mean \pm SD } \\
\hline 6 months & $0.37 \pm 0.38$ & $0.20 \pm 0.48$ & $0.42 \pm 0.39$ \\
\hline 12 months & $0.36 \pm 0.38$ & $0.31 \pm 0.42$ & $0.45 \pm 0.39$ \\
\hline \multicolumn{4}{|c|}{ Refractive astigmatism (D), mean } \\
\hline Preoperative & $-1.27 \pm 1.2$ & $-0.99 \pm 1.02$ & $-1.27 \pm 1.23$ \\
\hline 6 months & $-3.17 \pm 1.9^{\mathrm{a}}$ & $-1.53 \pm 1.44^{\mathrm{a}, \mathrm{b}}$ & $-1.92 \pm 1.1^{\mathrm{a}, \mathrm{c}}$ \\
\hline 12 months & $-2.95 \pm 1.96^{a}$ & $-1.47 \pm 1.32^{\mathrm{a}, \mathrm{b}}$ & $-2.01 \pm 2.01^{\mathrm{a}, \mathrm{c}}$ \\
\hline BSCVA gain 12 months, n (\%) & $7(17.5)$ & $7(19.4)$ & $4(9.5)$ \\
\hline$<0.0 \log M A R$ & $9(22.5)$ & $11(30.6)$ & $10(23.8)$ \\
\hline $0.0-0.2 \log M A R$ & $10(25)$ & $7(19.4)$ & $10(23.8)$ \\
\hline $0.2-0.4 \log M A R$ & $4(10)$ & $3(8.3)$ & $6(14.3)$ \\
\hline $0.4-0.6 \log M A R$ & $3(7 . .05)$ & $2(5.6)$ & $3(7.1)$ \\
\hline $0.6-0.8 \log M A R$ & $7(17.5)$ & $6(16.7)$ & $9(21.4)$ \\
\hline$>0.8 \log M A R$ & & & \\
\hline
\end{tabular}


TABLE.2 Preoperative and Postoperative Best Spectacle-Corrected Visual Acuity and Refractive Astigmatism (Pooled Data) for the 3 Treatment Groups (continued)

\begin{tabular}{llll}
\hline & PK & FS-DSEK & DSAEK \\
\hline $\begin{array}{l}\text { Refractive astigmatism at } 12 \\
\text { months, Cumulative } \mathbf{n}(\%)\end{array}$ & & & \\
$<$ 1D & $5(12.5)$ & $11(30.6)$ & $8(19.0)$ \\
$<$ 2D & $17(42.5)$ & $27(75.0)$ & $24(57.1)$ \\
$<3 D$ & $21(52.5)$ & $30(83.3)$ & $32(76.2)$ \\
$<4 D$ & $32(80.0)$ & $36(100.0)$ & $39(92.9)$ \\
\hline
\end{tabular}

BSCVA = best spectacle-corrected visual acuity; $\mathrm{D}=$ diopter; DSAEK = Descemet stripping automated endothelial keratoplasty; FS-DSEK = femtosecond laser-assisted Descemet stripping endothelial keratoplasty; logMAR = logarithm of the minimal angle of resolution; $\mathrm{PK}=$ penetrating keratoplasty; $\mathrm{SD}=$ standard deviation.

${ }^{a} P<.05$ between preoperative measurement and postoperative measurement.

${ }^{\mathrm{b}} P<.05$ between FS-DSEK and PK.

${ }^{c} P<.05$ between DSAEK and PK.

FS-DSEK group, and DSAEK group, respectively ( $P=$ not significant [NS]). At 6 and 12 months postoperatively, mean BSCVA was significantly better in the PK and DSAEK groups as compared to the FS-DSEK group. However, mean BSCVA gain was not significantly different. Preoperatively, mean refractive astigmatism was $-1.27 \pm 0.19 D,-0.99 \pm$ $0.17 \mathrm{D}$, and $-1.27 \pm 0.19 \mathrm{D}$ in the PK, FS-DSEK, and DSAEK groups, respectively $(P=N S)$. At 6 and 12 months postoperatively, mean refractive astigmatism was significantly lower in the FS-DSEK group and DSAEK group as compared to the PK group.

Table 3 shows the preoperative and postoperative mean composite score on the NEI-VFQ 25. Preoperatively, mean scores were 60.9, 58.2, and 56.1 in the PK, FS-DSEK, and DSAEK groups, respectively $(P=\mathrm{NS})$. At 6 and 12 months postoperatively, mean scores were found to be significantly higher in all groups as compared to the scores preoperatively. However, the change scores showed no significant differences between the groups.

At 12 months postoperatively, pooled data showed that $44 \%$ of the patients in the PK group were clinically improved (defined as both a clinically improved BSCVA and a clinically acceptable refractive astigmatism), $43 \%$ in the FS-DSEK group and $52 \%$ in the DSAEK group. 
TABLE 3. Preoperative and Postoperative Composite Scores on the National Eye Institute Visual Functioning Questionnaire (Pooled Data) for 3 Treatment Groups

\begin{tabular}{llll}
\hline & PK & FS-DSEK & DSAEK \\
\hline Preoperative & Mean \pm SD & Mean \pm SD & Mean \pm SD \\
6 months & $60.9 \pm 2.6$ & $58.2 \pm 3.0$ & $56.1 \pm 2.3$ \\
12 months & $69.8 \pm 2.7^{\mathrm{a}}$ & $67.2 \pm 3.4^{\mathrm{a}}$ & $69.3 \pm 1.7^{\mathrm{a}}$ \\
Change scores & $73.3 \pm 2.4^{\mathrm{a}}$ & $69.5 \pm 2.8^{\mathrm{a}}$ & $71.2 \pm 2.2^{\mathrm{a}}$ \\
T1 - T0 & & & \\
Change scores & $8.8 \pm 2.2$ & $9.0 \pm 2.4$ & $13.2 \pm 2.5$ \\
T2- T0 $^{\mathrm{b}}$ & & & \\
\hline
\end{tabular}

DSAEK = Descemet stripping automated endothelial keratoplasty; FS-DSEK = femtosecond laser-assisted Descemet stripping endothelial keratoplasty; $\mathrm{PK}=$ penetrating keratoplasty; $\mathrm{SE}=$ standard error of the mean.

${ }^{\mathrm{a}} \mathrm{P}<.05$ between preoperative measurement and postoperative measurement.

${ }^{\mathrm{b}} \mathrm{T} 0=$ preoperatively; $\mathrm{T} 1=6$ months postoperatively; $\mathrm{T} 2=12$ months postoperatively.

\section{Costs}

Table 4 shows the mean resource use and mean costs per patient. Mean total costs per patient were $€ 6674$ (US\$7942), $€ 12443$ (US\$14 807), and €7072 (US\$8416) in the PK group, FS-DSEK group, and DSAEK group, respectively. Differences in costs were mainly caused by the costs of the preparation of the posterior lamellar disc.

TABLE 4. Mean Resource Use and Mean Costs per Patient $(n=118)$ for the 3 Treatment Groups

\begin{tabular}{|c|c|c|c|c|c|c|c|}
\hline & \multicolumn{4}{|c|}{ Mean Resource Use } & \multicolumn{3}{|c|}{ Mean Costs \pm SD $(€)$} \\
\hline & $\begin{array}{l}\text { Costs Per } \\
\text { Unit }(€)\end{array}$ & $\begin{array}{c}\text { PK } \\
(n=40)\end{array}$ & $\begin{array}{c}\text { FS-DSEK } \\
(\mathrm{n}=36)\end{array}$ & $\begin{array}{l}\text { DSAEK } \\
(n=42)\end{array}$ & $\begin{array}{c}\text { PK } \\
(n=40)\end{array}$ & $\begin{array}{l}\text { FS-DSEK } \\
(n=36)\end{array}$ & $\begin{array}{l}\text { DSAEK } \\
(n=42)\end{array}$ \\
\hline \multicolumn{8}{|l|}{ Preoperative costs } \\
\hline BISLIFE foundation services & 3747/cornea & 1 & 1 & 1 & 3747 & 3747 & 3747 \\
\hline Out-patient visits & 27/visit & 0.7 & 0.6 & 1 & 19 & 18 & 28 \\
\hline Diagnostic procedures & Variable & 0.7 & 1.4 & 0.5 & 13 & 26 & 8 \\
\hline Subtotal preoperative costs & & & & & 3779 & 3790 & 3783 \\
\hline \multicolumn{8}{|l|}{ Surgical procedures } \\
\hline \multicolumn{8}{|l|}{ Preparation PLD } \\
\hline Costs femto-second laser & 4821/lamel & - & 1 & - & - & 4821 & - \\
\hline $\begin{array}{l}\text { Costs microkeratome } \\
\text { transplantation }\end{array}$ & 433/lamel & - & - & 1 & - & - & 433 \\
\hline Operating room costs & $9.68 /$ minute & 103 & 139 & 126 & 999 & 1343 & 1217 \\
\hline Ophthalmology costs & $6.30 /$ minute & 73 & 95 & 88 & 463 & 596 & 554 \\
\hline Intraocular lens & 138/lens & 0.3 & 0.1 & 0.1 & 45 & 19 & 18 \\
\hline
\end{tabular}


TABLE 4. Mean Resource Use and Mean Costs per Patient $(n=118)$ for the 3 Treatment Groups (continued)

\begin{tabular}{|c|c|c|c|c|c|c|c|}
\hline & \multirow[b]{2}{*}{$\begin{array}{l}\text { Costs Per } \\
\text { Unit }(€)\end{array}$} & \multicolumn{3}{|c|}{ Mean Resource Use } & \multicolumn{3}{|c|}{ Mean Costs \pm SD (€) } \\
\hline & & $\begin{array}{c}\text { PK } \\
(n=40)\end{array}$ & $\begin{array}{c}\text { FS-DSEK } \\
(\mathrm{n}=36)\end{array}$ & $\begin{array}{l}\text { DSAEK } \\
(n=42)\end{array}$ & $\begin{array}{c}\text { PK } \\
(n=40)\end{array}$ & $\begin{array}{l}\text { FS-DSEK } \\
(n=36)\end{array}$ & $\begin{array}{r}\text { DSAEK } \\
(n=42)\end{array}$ \\
\hline \multicolumn{8}{|l|}{ Additional procedures } \\
\hline Operating room costs & 9.68/minute & 25 & 38 & 2 & 246 & 371 & 22 \\
\hline Ophthalmology costs & $6.30 /$ minute & 8 & 20 & 1 & 50 & 127 & 8 \\
\hline Subtotal surgical procedures & & & & & 1802 & 7277 & 2252 \\
\hline \multicolumn{8}{|l|}{ Hospitalisation } \\
\hline Day care & 232/day & 0.3 & - & - & 70 & - & - \\
\hline Admission & 252/day & 2.4 & 3.7 & 3.1 & 557 & 865 & 732 \\
\hline Subtotal hospitalization & & & & & 627 & 865 & 732 \\
\hline \multicolumn{8}{|l|}{ Follow-up visits } \\
\hline Outpatient visits & 27/visit & 11 & 10.3 & 7.1 & 301 & 280 & 194 \\
\hline Diagnostic procedures & Variable & 6.2 & 10.6 & 4.9 & 124 & 191 & 71 \\
\hline Subtotal follow-up visits & & & & & 425 & 471 & 265 \\
\hline \multirow[t]{2}{*}{ Postoperative drugs } & 10/bottle & 4 & 4 & 4 & 40 & 40 & 40 \\
\hline & & & & & 6674 & 12443 & 7072 \\
\hline Total costs (€) & & & & & \pm 82 & \pm 97 & \pm 30 \\
\hline
\end{tabular}

BIS = Bio Implant Services; DSAEK = Descemet stripping automated endothelial keratoplasty; FS-DSEK = femtosecond laser-assisted Descemet stripping endothelial keratoplasty; PK = penetrating keratoplasty; PLD = posterior lamellar disk.

\section{Cost-effectiveness}

Table 5 shows the results of the primary analysis. As FS-DSEK was less effective and more costly for costs per clinically improved patient, FS-DSEK was dominated by DSAEK and PK. For DSAEK vs PK, the ICER was $€ 4975$ (US\$5920) per clinically improved patient. The probability of DSAEK being cost-effective ranged from $22 \%$ at a ceiling ratio of $€ 2500$ (US\$2975) to $68 \%$ at a ceiling ratio of $€ 10000$ (US\$11 900).

In the Figure, the CEACs for the primary analysis are displayed, showing a probability of $0 \%$ that FS-DSEK is cost-effective for the full range of ceiling ratios. The cost-effectiveness acceptability frontier (not shown) showed that PK has the highest probability of being cost-effective until the ceiling ratio reached $€ 5115$ (US\$6087) and DSAEK has the highest probability of being cost-effective for all values above $€ 5115$ (US\$6087).

\section{Secondary analyses}

In Table 5, the results of the secondary analyses are shown. In all secondary analyses, FS-DSEK was dominated by DSAEK and PK or extended dominated by DSAEK. In the secondary analyses with other outcome measures, the ICERs for DSAEK vs PK were $€ 13$ 267 (US\$15 788) per improved patient on the NEI VFQ-25 and $€ 3980$ (US\$4736) per improved patient on all 3 outcome measures. 


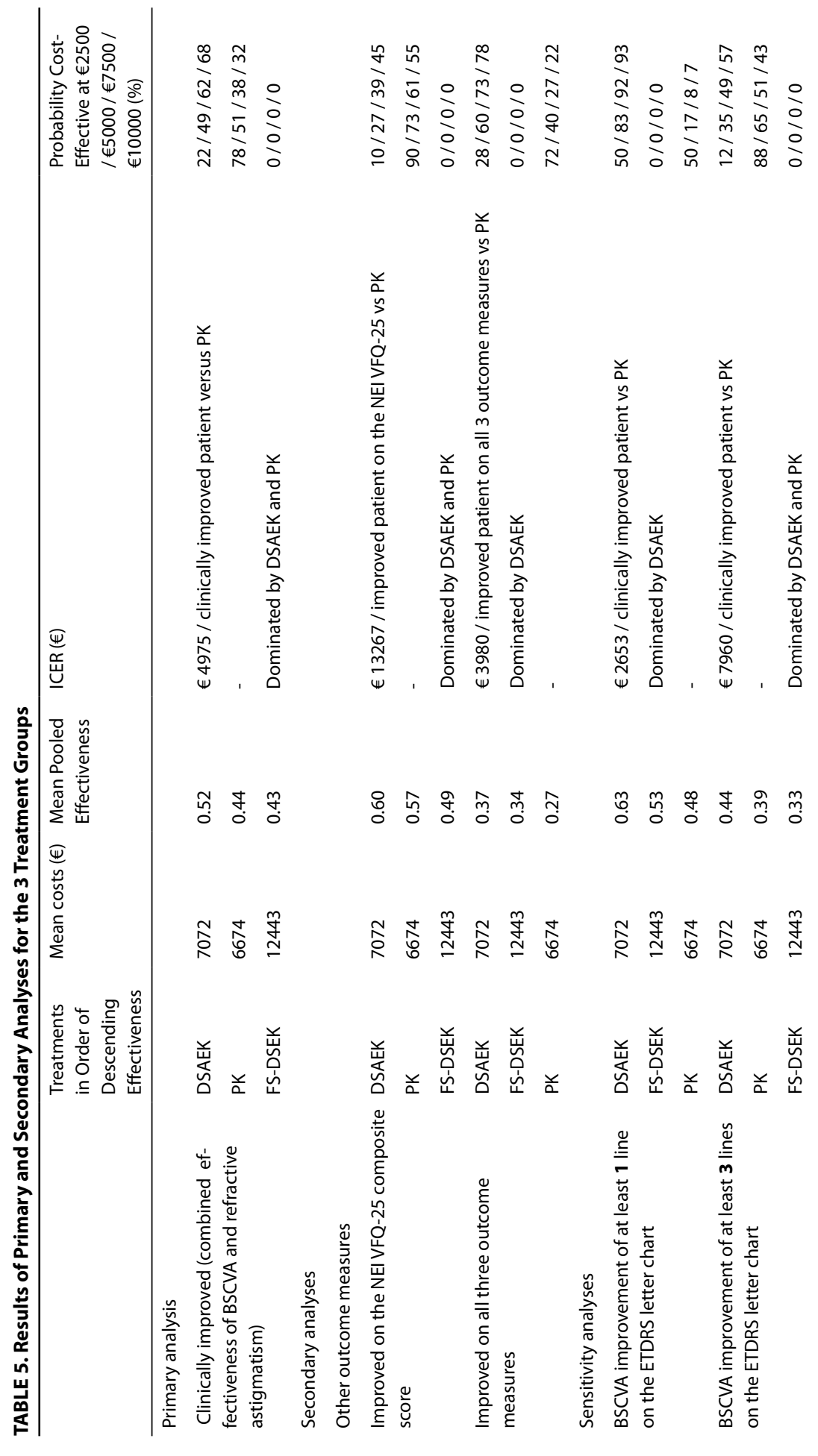




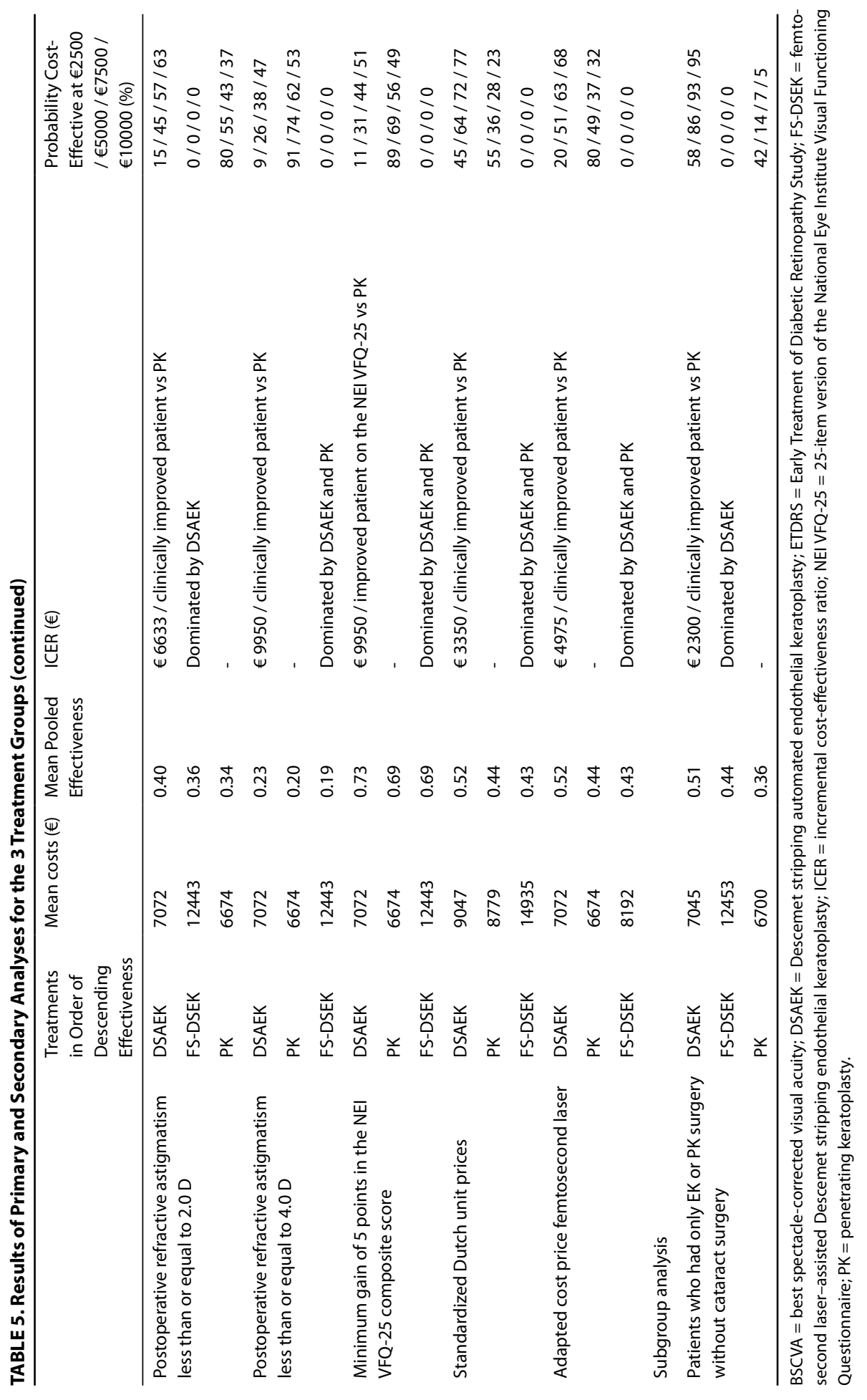




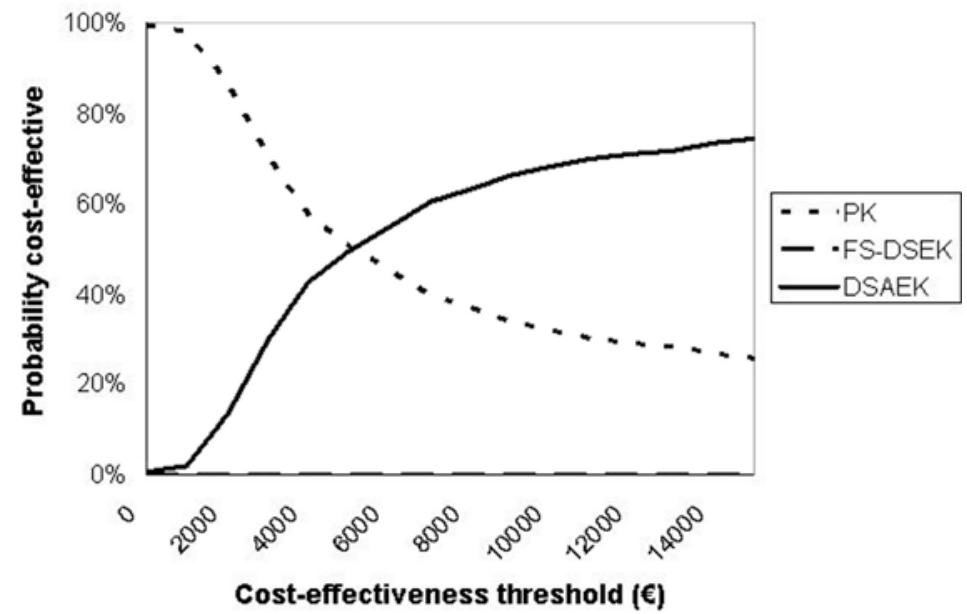

FIGURE. Cost-effectiveness acceptability curves for the incremental costs per clinically improved patient for penetrating keratoplasty (PK), femtosecond laser-assisted Descemet stripping endothelial keratoplasty (FS-DSEK), and Descemet stripping automated endothelial keratoplasty (DSAEK). The curve shows the probability (vertical axis) of which technique is cost-effective over a range of values for the maximum acceptable ceiling ratio (horizontal axis).

In the sensitivity analyses, the ICERs for DSAEK vs PK varied from $€ 2653$ (US\$3157) to $€ 9950$ (US\$11 840) per improved patient, showing that the main cost-effectiveness data are robust.

In the subgroup analysis, we analyzed the subgroup of patients who had only a PK ( $\mathrm{n}=$ $27)$, FS-DSEK $(n=31)$, or DSAEK $(n=37)$ without cataract surgery. The ICER for DSAEK vs PK was $€ 2300$ (US\$2737) per clinically improved patient.

\section{DISCUSSION}

This economic evaluation compared the cost-effectiveness of 2 different techniques of endothelial keratoplasty (FS-DSEK and DSAEK) and penetrating keratoplasty (PK). The results show that based on the point estimates FS-DSEK was dominated by DSAEK and PK or extended dominated by DSAEK for all outcome measures. DSAEK was found to be more costly, but also more effective on all outcome measures, as compared to PK. The base-case bootstrap analysis and secondary analyses showed that results were consistent in that FS-DSEK has a very low to zero probability of being a cost-effective technique compared to DSAEK and PK. At a lower range of threshold values for costeffectiveness PK has the highest probability of being cost-effective, whereas at a higher range of threshold values DSAEK has the highest probability of being cost-effective. In between a range of $€ 2500$ (US\$2975) and $€ 7500$ (US\$8925), the uncertainty around the 
ICERs is quite large, as within this range of threshold values probabilities vary between $40 \%$ and $60 \%$.

Economic evaluations aim to facilitate decisions on allocation of resources by policymakers and insurers. The data on clinical outcomes used in economic evaluations can be drawn from clinical trials, but it is not uncommon that the complete dataset may have to be drawn from a wider set of study designs. ${ }^{36}$ One type of economic evaluation is the cost-utility analysis, in which costs of 2 or more alternatives are expressed in monetary terms and the effectiveness is expressed in a measure that comprises both quality and length of life. ${ }^{37}$ For this purpose, quality-adjusted life years (QALYs) are calculated. As a QALY is a generic outcome measure, it facilitates comparisons of different interventions in health care. Threshold values for cost-effectiveness can be used to make decisions on the allocation of resources. In The Netherlands, threshold values range from $€ 20000$ (US\$23 800) per QALY to €80 000 (US\$95 200) per QALY. ${ }^{38}$

Beauchemin and associates evaluated the cost-utility of EK compared to PK in a hypothetical cohort of 100 patients waiting for a corneal graft. ${ }^{39}$ They constructed a Markov model to analyze the cost-utility from a health system perspective over a lifetime period. EK was more effective and less costly compared to PK. The differences between the 2 treatments were mainly caused by a lower incidence of irreversible graft failure and postoperative complications in EK patients.

In a cost-effectiveness study, costs of 2 or more alternatives are expressed in monetary terms and effects are expressed in natural health units, depending on the interventions being evaluated. ${ }^{40}$ In this study, we choose to measure the effectiveness in 3 ways, namely clinically improved BSCVA (defined as an improvement of at least 2 lines), clinically acceptable refractive astigmatism (defined as less than or equal to $3.0 \mathrm{D}$ ), and improved vision-related quality of life (a minimum gain of 10 points in the composite score of the NEI VFQ-25). We acknowledge that there will be patients who have a clinically improved BSCVA and a clinically acceptable refractive astigmatism, but who are not improved in terms of quality of life. However, several studies ${ }^{16,22,23}$ used these outcome measures and we believe that not only the subjective quality of life but also the more objective BSCVA and refractive astigmatism are important outcome measures in patients who undergo corneal transplantation.

Contrary to cost-utility analysis with QALYs as an outcome measure, the interpretation of the cost-effectiveness with these outcome measures is difficult, as it is not known what a hospital, health insurance company, or government is willing to pay for an improved patient. Furthermore, as no other studies are performed that evaluated cost-effectiveness using these outcome measures, it is hard to relate our results to other studies.

The ICER for DSAEK vs PK was €4975 (US\$5920) per clinically improved patient. Although comparison with a threshold value of $€ 20000$ (US\$23 800) per QALY is dif- 
ficult, this ICER is far below this threshold and seems to be an acceptable price to pay for an additional improved patient.

In EK patients, a large part of the mean costs per patient is associated with the preparation of the PLDs, which is performed with a femtosecond laser in the FS-DSEK group and a microkeratome in the DSAEK group. In the sensitivity analyses, it was shown that the cost of the laser can be lowered to $€ 8192$ (US\$9748) when including additional procedures. In the future, the transplants can be produced under standardized conditions in a national cornea bank and the present infrastructure can be used to distribute the lamellar transplant buttons to transplanting surgeons all over The Netherlands. This can significantly lower the mean costs per patient for the preparation of the lamellar disks in FS-DSEK and DSAEK patients.

Some limitations of this study need to be addressed. First, the patients in the DSAEK group were included based on the completion of quality-of-life questionnaires. In Maastricht University Medical Center it is the standard of care to distribute quality-oflife questionnaires preoperatively and postoperatively to patients who receive a corneal transplantation. However, some patients forget to send back the completed questionnaires. Therefore, only 42 of the potential 73 DSAEK patients (58\%) could be included in the study. Since the inclusion criteria are equal for all groups in the study and baseline characteristics are comparable, we believe no selection bias is present.

Second, we included only costs within the health care sector. The costs associated with the correction of astigmatism (for example, spectacles or contact lenses with cylinder correction) were not included. As the mean refractive astigmatism is significantly lower in the FS-DSEK group and DSAEK group as compared to the PK group, it can be assumed that the mean costs per patient for the correction of astigmatism will be lower in the EK groups. Furthermore, the costs of productivity losses were not assessed. As EK shows more rapid freedom from work-related activity restriction and faster recovery of useful vision as compared to PK, these costs may be lower in the EK groups.

Third, the follow-up period of the study was only 12 months. Therefore, this study only provides an indication of the short-term cost-effectiveness of EK vs PK. Although costs within 1 year after surgery were higher in EK patients, it could be argued that long-term costs are lower and long-term effects are higher as compared to PK, because of the effects of suture removal in the PK group and a reduced risk of complications in the EK groups (eg, wound dehiscence and infectious keratitis), with fewer hospital visits and emergency surgical procedures to be expected. ${ }^{7,41,42}$ This will have a positive effect on the long-term cost-effectiveness of EK. However, a longer follow-up period is needed to evaluate this effect further.

The results of this study show that FS-DSEK was not cost-effective compared to PK and DSAEK. DSAEK, on the other hand, was more costly and also more effective, resulting in an ICER of $€ 4975$ (US\$5920) per clinically improved patient. It depends on the threshold 
value for cost-effectiveness whether DSAEK or PK is the preferred technique. Within a broad range of threshold values both DSAEK and PK might be cost-effective. However, preparing lamellar transplant buttons in a national cornea bank can significantly lower the costs per patient in the DSAEK group, which could improve the cost-effectiveness of DSAEK and could lower the uncertainty around the ICERs. Studies with a longer followup period are necessary to evaluate the long-term cost-effectiveness. 


\section{REFERENCES}

1. Thompson RW Jr, Price MO, Bowers PJ, Price FW Jr. Long-term graft survival after penetrating keratoplasty. Ophthalmology 2003;110(7):1396-1402.

2. Beckingsale $P$, Mavrikakis I, Al-Yousuf N, Mavrikakis E, Daya SM. Penetrating keratoplasty: outcomes from a corneal unit compared to national data. Br J Ophthalmol 2006;90(6):728 -731.

3. Williams KA, Esterman AJ, Bartlett C, Holland H, Hornsby NB, Coster DJ. How effective is penetrating corneal transplantation? Factors influencing long-term outcome in multivariate analysis. Transplantation 2006;81(6):896 -901.

4. Gorovoy MS. Descemet-stripping automated endothelial keratoplasty. Cornea 2006;25(8):886-889.

5. Terry MA, Ousley PJ. Deep lamellar endothelial keratoplasty in the first United States patients: early clinical results. Cornea 2001;20(3):239 -243.

6. Price FW Jr, Price MO. Descemet's stripping with endothelial keratoplasty in 200 eyes: Early challenges and techniquesto enhance donor adherence. J Cataract Refract Surg 2006; 32(3):411- 418.

7. Bahar I, Kaiserman I, McAllum P, Slomovic A, Rootman D. Comparison of posterior lamellar keratoplasty techniques to penetrating keratoplasty. Ophthalmology 2008;115(9): 1525-1533.

8. Koenig SB, Covert DJ, Dupps WJ Jr, Meisler DM. Visual acuity, refractive error, and endothelial cell density six months after Descemet stripping and automated endothelial keratoplasty (DSAEK). Cornea 2007;26(6):670-674.

9. Terry MA, Chen ES, Shamie N, Hoar KL, Friend DJ. Endothelial cell loss after Descemet's stripping endothelial keratoplasty in a large prospective series. Ophthalmology 2008;115(3):488-496.e483.

10. Mearza AA, Qureshi MA, Rostron CK. Experience and 12-month results of descemet-stripping endothelial keratoplasty (DSEK) with a small-incision technique. Cornea 2007;26(3):279 -283.

11. Cheng YY, Tahzib NG, van Rij G, et al. Femtosecond laser-assisted inverted mushroom keratoplasty. Cornea 2008(6);27:679-685.

12. Price MO, Baig KM, Brubaker JW, Price FW Jr. Randomized, prospective comparison of precut vs surgeon-dissected grafts for descemet stripping automated endothelial keratoplasty. Am J Ophthalmol 2008(1);146:36-41.

13. Cheng $Y Y$, Hendrikse F, Pels $E$, et al. Preliminary results of femtosecond laser-assisted descemet stripping endothelial keratoplasty. Arch Ophthalmol 2008;126(10):1351-1356.

14. Cheng YY, Kang SJ, Grossniklaus HE, et al. Histologic evaluation of human posterior lamellar discs for femtosecond laser Descemet's stripping endothelial keratoplasty. Cornea 2009;28(1):73-79.

15. Cheng YY, Pels E, Nuijts RM. Femtosecond-laser-assisted Descemet's stripping endothelial keratoplasty. J Cataract Refract Surg 2007;33(1):152-155.

16. Cheng YY, Schouten JS, Tahzib NG, et al. Efficacy and safety of femtosecond laser-assisted corneal endothelial keratoplasty: a randomized multicenter clinical trial. Transplantation 2009;88(11):1294 -1302 .

17. McLaren JW, Patel SV, Bourne WM, Baratz KH. Corneal wavefront errors 24 months after deep lamellar endothelial keratoplasty and penetrating keratoplasty. Am J Ophthalmol 2009;147(6):959 $-965,965 . e 1-2$.

18. Patel SV, McLaren JW, Hodge DO, Baratz KH. Scattered light and visual function in a randomized trial of deep lamellar endothelial keratoplasty and penetrating keratoplasty. Am J Ophthalmol 2008;145(1):97-105.

19. Drummond MF, Sculpher MJ, Torrance GW, O'Brien BJ, Stoddart GL. Methods for the Economic Evaluation of Health Care Programmes. 3rd ed. Oxford: Oxford University Press; 2005. 
20. Cheng YY, van Den Berg TJ, Schouten JS, et al. Quality of vision after femtosecond laser-assisted Descemet stripping endothelial keratoplasty and penetrating keratoplasty: a randomized, multicenter clinical trial. Am J Ophthalmol 2011; 152(4):556 -566.

21. Ferris FL 3rd, Kassoff A, Bresnick GH, Bailey I. New visual acuity charts for clinical research. Am J Ophthalmol 1982; 94(1):91-96.

22. McKean-Cowdin R, Varma R, Hays RD, Wu J, Choudhury F, Azen SP. Longitudinal changes in visual acuity and health-related quality of life: the Los Angeles Latino Eye Study. Ophthalmology 2010;117(10):1900 -1907, 1907.e1.

23. Submacular Surgery Trials Research Group. Evaluation of minimum clinically meaningful changes in scores on the National Eye Institute Visual Function Questionnaire (NEIVFQ) SST Report Number 19. Ophthalmic Epidemiol 2007; 14(4):205-215.

24. Frost NA, Wu J, Lai TF, Coster DJ. A review of randomized controlled trials of penetrating keratoplasty techniques. Ophthalmology 2006;113(6):942-949.

25. Lindblad AS, Clemons TE. Responsiveness of the National Eye Institute Visual Function Questionnaire to progression to advanced age-related macular degeneration, vision loss, and lens opacity: AREDS Report no. 14. Arch Ophthalmol 2005;123(9):1207-1214.

26. Miskala PH, Bass EB, Bressler NM, et al. Surgery for subfoveal choroidal neovascularization in age-related macular degeneration: quality-of-life findings: SST report no. 12. Ophthalmology 2004;111(11):1981-1992.

27. Miskala PH, Hawkins BS, Mangione CM, et al. Responsiveness of the National Eye Institute Visual Function Questionnaire to changes in visual acuity: findings in patients with subfoveal choroidal neovascularization-SST Report No. 1. Arch Ophthalmol 2003;121(4):531-539.

28. Oostenbrink JB, Bouwmans CAM, Koopmanschap MA, Rutten FFH. Handleiding voor Kostenonderzoek: Methoden en Standaard Kostprijzen voor Economische Evaluaties in de Gezondheidszorg.Diemen: College voor Zorgverzekeringen; 2010.

29. Organization for Economic Co-operation and Development. Table 4: PPP and Exchange Rates. Available at: http://Stats.Oecd.Org/Index.Aspx?DatasetcodeSna_Table4. Accessed: January 7, 2012.

30. Dutch Health Care Insurance Board. Pharmacotherapeutic Compass [in Dutch]. Amstelveen: College voor Zorgverzekeringen; 2008

31. Barber JA, Thompson SG. Analysis of cost data in randomized trials: an application of the nonparametric bootstrap. Stat Med 2000;19(23):3219 -3236.

32. Postma MJ, de Vries R, Welte R, Edmunds WJ. Health economic methodology illustrated with recent work on Chlamydia screening: the concept of extended dominance. Sex Transm Infect 2008;84(2):152-154.

33. Glick HA, Doshi J, Sonnad S, Polsky D. Economic evaluation in clinical trials. New York: Oxford University Press; 2007:133-144.

34. Fenwick E, O'Brien BJ, Briggs A. Cost-effectiveness acceptability curves-facts, fallacies and frequently asked questions. Health Econ 2004;13(5):405- 415

35. Fenwick E, Claxton K, Sculpher M. Representing uncertainty: the role of cost-effectiveness acceptability curves. Health Econ 2001;10(8):779 -787.

36. Drummond MF, Sculpher MJ, Torrance GW, O'Brein BJ, Stoddart GL. Methods for Economic Evaluation of Health Care Programmes. 3rd ed. Oxford: Oxford University Press; 2005:105.

37. Brown MM, Brown GC, Sharma S, Landy J. Health care economic analyses and value-based medicine. Surv Ophthalmol 2003;48(2):204 -223. 
38. Council for Public Health and Health Care. Sensible and sustainable care [in Dutch]. The Netherlands: Den Haag; 2006.

39. Beauchemin C, Brunette I, Boisjoly H, Freeman EE, Popescu M, Lachaine J. Economic impact of the advent of posterior lamellar keratoplasty in Montreal, Quebec. Can J Ophthalmol 2010;45(3):243251.

40. van den Biggelaar FJ, Cheng YY, Nuijts RM, et al. Economic evaluation of deep anterior lamellar keratoplasty versus penetrating keratoplasty in The Netherlands. Am J Ophthalmol 2011(3);151:449459.e442.

41. Price MO, Jordan CS, Moore G, Price FW Jr. Graft rejection episodes after Descemet stripping with endothelial keratoplasty: part two: the statistical analysis of probability and risk factors. $\mathrm{Br} J$ Ophthalmol 2009;93(3):391-395.

42. Allan BD, Terry MA, Price FW Jr, Price MO, Griffin NB, Claesson M. Corneal transplant rejection rate and severity after endothelial keratoplasty. Cornea 2007;26(9):1039-1042. 


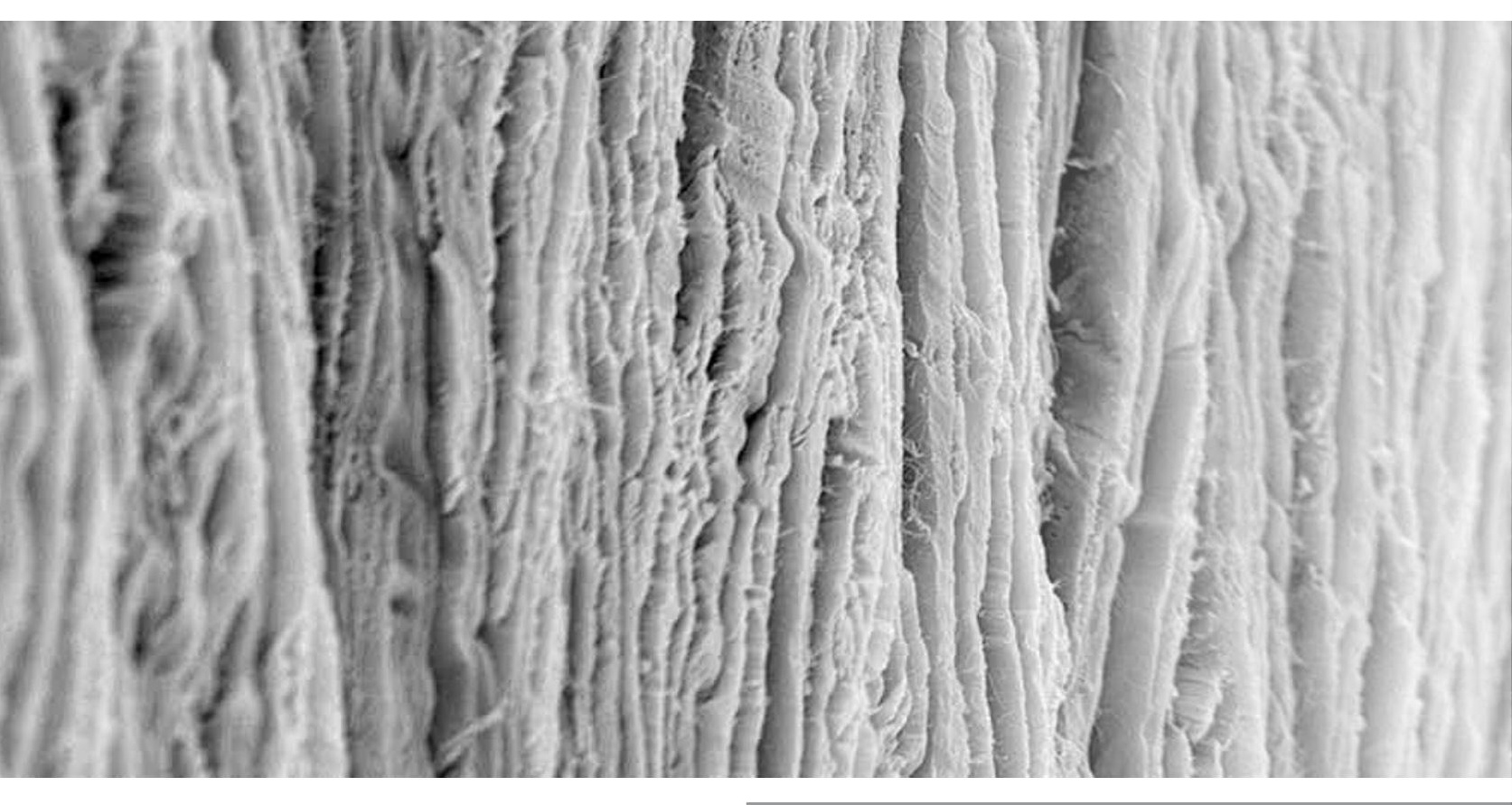




\title{
CHAPTER 9
}

Femtosecond Laser-assisted Inverted Mushroom Keratoplasty

\author{
Yanny Y. Y. Cheng \\ Nayyirih G. Tahzib \\ Gabriel van Rij \\ Hugo van Cleynenbreugel \\ Elisabeth Pels \\ Fred Hendrikse \\ Rudy M.M.A. Nuijts
}

Cornea. 2008 Jul;27(6):679-85 


\section{ABSTRACT}

Purpose: To evaluate best-corrected visual acuity (BCVA), refractive outcome, corneal topography, optical coherence tomography, and endothelial cell density 12 months after femtosecond laser-assisted inverted mushroom keratoplasty.

Methods: We performed a prospective study of a surgical case series of 5 patients undergoing femtosecond laser-assisted inverted mushroom keratoplasty for pseudophakic bullous keratopathy or pre-Descemet $X$-linked ichthyosis. The femtosecond laser was used to create a top-hat configuration in the donor cornea and recipient cornea. Laser parameters were as follows: energy, 4.0 (anterior inner vertical side cut and horizontal lamellar cut) and $7.0 \mu \mathrm{J}$ (posterior outer vertical side cut); spiral pattern with a firing rate of $15 \mathrm{kHz}$. The size of the anterior inner diameter was $7.4 \mathrm{~mm}$ in the donor cornea and 7.0 $\mathrm{mm}$ in the recipient cornea. The posterior outer diameter was $9.0 \mathrm{~mm}$ in all eyes.

Results: At 6 and 12 months after surgery, all corneal grafts were clear and showed an excellent adaptation of the lamellar donor and recipient wound surfaces. At 12 months post-operatively, BCVA averaged 20/32 (range, 20/60-20/20), refractive cylinder averaged $-3.20 \pm 2.0 \mathrm{D}$, topographical cylinder averaged $3.26 \pm 2.1 \mathrm{D}$, and the mean endothelial cell density was $1793 \pm 491$ cells $/ \mathrm{mm}^{2}$ (range, 954-2237 cells $/ \mathrm{mm}^{2}$ ). The mean central corneal thickness and thickness of the posterior shelf was $517 \pm 3$ and 175 $\pm 8 \mu \mathrm{m}$, respectively.

Conclusions: The femtosecond laser-assisted inverted mushroom keratoplasty shows good promise in surgical treatment of corneal diseases. The multiplanar fit between the donor and recipient cornea allows early suture removal and visual rehabilitation. 


\section{INTRODUCTION}

Full-thickness penetrating keratoplasty has been the preferred corneal transplantation technique since the first description of successful corneal transplantation in 1905 by Zirm. ' In penetrating keratoplasty, the recipient cornea is trephined in the vertical direction, and the donor cornea is sutured into the wound bed by using a variety of suture techniques. Although penetrating keratoplasty nowadays generally results in clear corneal grafts with a graft survival up to $72 \%$ at 5 years, ${ }^{2,3}$ the procedure is frequently complicated by refractive imperfections and wound healing problems. ${ }^{4,5}$ Postoperative high irregular astigmatism and ametropia may result in many patients that cannot be optically rehabilitated with glasses. Treatment modalities for postkeratoplasty astigmatism and ametropia are rigid gas-permeable contact lens wear, corneal laser refractive surgery, and implantation of toric intraocular lenses. ${ }^{6-8}$ Wound healing after penetrating keratoplasty is often unstable and may lead to infection, vascularization, and wound dehiscence with risks for long-term graft survival. ${ }^{9,10}$

Recently, Busin ${ }^{11}$ has made popular the use of a so-called inverted mushroom lamellar dissection technique for creating a top-hat configuration of the donor. This technique may result in faster healing of the lamellar wound surfaces that facilitates earlier suture removal and a faster visual rehabilitation of the corneal graft patient.

We describe a new method of preparing a top-hat graft and cutting the recipient bed in a standardized and automated fashion by using a femtosecond laser, thereby introducing femtosecond laser technology in penetrating keratoplasty surgery.

\section{MATERIALS AND METHODS}

Three patients with pseudophakic bullous keratopathy and 2 patients with pre-Descemet X-linked ichthyosis underwent femtosecond laser-assisted inverted mushroom keratoplasty. Investigational review board approval for this study was obtained from the University Hospital Maastricht, and informed consent was obtained from the patients. All procedures were performed in the University Hospital Maastricht by the same surgeon (R.N.), and the patients were prospectively followed.

Preoperatively, the medical history of each patient was recorded, and a complete eye examination was performed including visual acuity testing, slit-lamp examination, and ophthalmoscopy. Postoperatively, patients were seen at 1 week and 1, 3, 6, and 12 months. From 1 month after surgery, best-corrected visual acuity (BCVA) was measured, and the refractive outcome and corneal topography parameters (Eyemap EH-290; Alcon, Fort Worth, TX) were determined. Specular microscopy (Konan Noncon Robo, SP 8000; Konan, Hyogo, Japan) for measurement of endothelial cell density was performed at 
6 and 12 months after surgery. Suture removal was performed at the discretion of the surgeon to decrease induced corneal astigmatism. The preoperative central and midperipheral corneal thickness of the recipient eyes were measured by ultrasonic pachymetry (Quantel Medical). The estimated thickness of the posterior shelf and the measured thickness of the central and midperipheral corneal graft were determined by optical coherence tomography (Visante OCT; Carl Zeiss, Oberkochen, Germany) at 1 year postoperatively.

General anesthesia was used in 4 patients, and in 1 patient, local anesthesia was administered with a retrobulbar injection of a mixture of $2 \%$ lidocaine and $0.5 \%$ bupivacainehydrochloride. We used organ-cultured donor corneas that were obtained from the Cornea Bank Amsterdam.

A femtosecond laser (AMO-IntraLase Corp., Irvine, CA) was installed in the operating theatre and equipped with a software package for producing custom-made corneal incisions in the donor and recipient corneas. A so-called top-hat configuration in the recipient cornea and in the donor cornea was created in all 5 patients (Fig. 1). The tophat configuration consists of an anterior inner vertical side cut, a horizontal lamellar cut, and a posterior outer vertical side cut. The vertical cuts have an overlap of $0.1 \mathrm{~mm}$ and an angulation of $90^{\circ}$ with the corneal surface to assure that complete cutting at the intersecting wound edges is performed. The dimensions of the cut are shown in Figure 2. For the donor cornea, in all cases the diameter of the anterior inner vertical side cut was 7.4 $\mathrm{mm}$, and the diameter of the posterior outer vertical side cut was $9.0 \mathrm{~mm}$. Preoperative corneal thickness was used for establishing the cutting dimensions of the femtosecond laser. The goal for the thickness of the posterior shelf (distance between endothelium and horizontal lamellar interface) was $200 \mu \mathrm{m}$. The energy level of the femtosecond laser for preparation of the anterior inner vertical side cut and the horizontal lamellar cut was $4.0 \mu \mathrm{J}$ and for the posterior outer vertical side cut was $7.0 \mu \mathrm{J}$, respectively. We used a spiral spot pattern with a firing rate of $15 \mathrm{kHz}$. The laser starts cutting in the anterior chamber at a depth of $\sim 900 \mu \mathrm{m}$ from the epithelial side and first makes the posterior outer vertical side cut at $9.0 \mathrm{~mm}$ diameter for an estimated thickness of $200 \mu \mathrm{m}$ for the posterior shelf. The horizontal lamellar side cut is created from 9.0 to $7.0 \mathrm{~mm}$ inward.
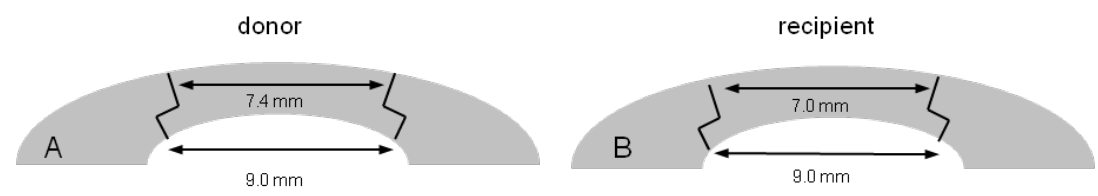

FIGURE 1. Schematic overview of the top-hat configuration in the donor cornea and recipient cornea. The diameter of the anterior inner vertical side cut of the donor cornea was 7.4 (A) and $7.0 \mathrm{~mm}$ in the recipient (B). The posterior outer diameter was $9.0 \mathrm{~mm}$ in all corneas. 


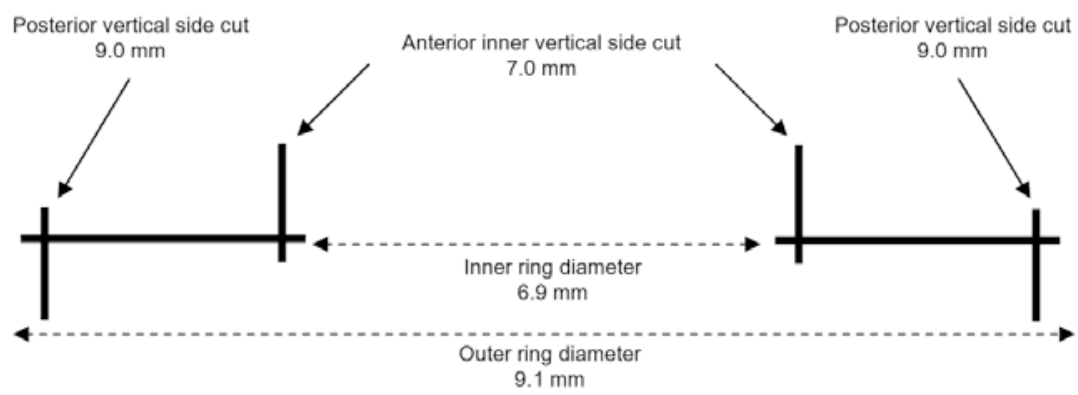

FIGURE 2. The setting of the femtosecond laser to create a top-hat configuration in the recipient cornea. The vertical cuts have an overlap of $0.1 \mathrm{~mm}$ with the horizontal lamellar cut to assure a complete cutting.

Finally, the anterior vertical side cut is created until the gas bubbles escape from the epithelial side of the cornea. The duration for the laser procedure was $\sim 2.5$ minutes.

The donor button was mounted on an artificial anterior chamber (Moria, Anthony, France) and perfused with a solution of balanced salt solution (BSS Plus; Alcon) after a viscoelastic substance (Healon; AMO, Uppsala, Sweden) was placed on the endothelium. The eye was pressurized, and the intraocular pressure was checked with a Barraquer tonometer (Bausch and Lomb, Munich, Germany). Pachymetry measuring central and midperipheral corneal thickness was performed to calculate the depth of the horizontal lamellar plane. The center of the cornea was marked with a $7.0-\mathrm{mm}$ trephine to guard a symmetric applanation by the applanation cone of the laser. No suction ring was needed in the donor eyes. After the laser procedure, the corneal graft was lifted from the host cornea, and isolated tissue bridges were excised.

In the recipient cornea, a suction ring was applied, and an applanation lens was used; then, the laser procedure was performed. After the injection of a viscoelastic substance into the anterior chamber, the cornea was dissected, and adhesions were gently removed. The corneal graft was sutured by using eight 10-0 nylon interrupted sutures in combination with an 11-0 nylon 24-bite continuous suture. Selective suture removal was used starting at 3 months postoperatively. The postoperative regimen consisted of dexamethasone $0.1 \%$ drops 6 times daily in a tapering dose for the first 6 months and thereafter 1 time daily for the first postoperative year. Chloramphenicol $0.4 \%$ drops were used 3 times daily for 3 months and then discontinued. 


\section{RESULTS}

The results are presented as mean \pm SD. In Table 1, the demographic and preoperative data of the patients are shown. The age varied from 42 to 75 years. Preoperative BCVA ranged from hand motions to $20 / 30$. Preoperatively, the mean endothelium cell density of the donor corneas was $2680 \pm 45$ cells $/ \mathrm{mm}^{2}$ (range, $2600-2700$ cells $/ \mathrm{mm}^{2}$ ). No operative complications were noted.

Three of the 5 patients (cases 2, 3, and 5) complained of photophobia during the first postoperative month. All 3 cases showed an increased level of stromal edema centrally and at the peripheral interface that gradually resolved (Fig. 3).

At 6 months postoperatively, the mean BCVA was 20/50 (range, 20/40-20/60). In 3 eyes, the postoperative BCVA was limited by age-related macular degeneration (case 3), cellophane maculopathy (case 4), and amblyopia (case 5 ). The mean refractive cylinder was $-4.75 \pm 2.4 \mathrm{D}$ (range, -2.0 to $-8.0 \mathrm{D}$ ). The mean topographic cylinder was $5.3 \pm 3.5 \mathrm{D}$ (range, 2.1-10.3 D). The mean endothelial cell density at 6 months postoperatively was $1917 \pm 155$ cells $/ \mathrm{mm}^{2}$ (range, 1751-2117 cells $/ \mathrm{mm}^{2}$ ). The central corneal thickness was $513 \pm 46 \mu \mathrm{m}$ (range, 474-575 $\mu \mathrm{m}$ ).

The 12-month postoperative data are shown in Table 2. All corneal grafts were clear and showed an excellent adaptation of the lamellar donor and recipient wound surfaces (Figs. 4 and 5). At 12 months, the mean BCVA was 20/32 (range, 20/60-20/20). The mean refractive cylinder was $-3.20 \pm 2.0 \mathrm{D}$ (range, -0.75 to $-5.5 \mathrm{D}$ ). The mean topographic cylinder was $3.26 \pm 2.1 \mathrm{D}$ (range, $0.6-5.6 \mathrm{D}$ ). The mean endothelial cell density at 12 months post-operatively was $1793 \pm 491$ cells $/ \mathrm{mm}^{2}$ (range, $954-2237$ cells $/ \mathrm{mm}^{2}$ ). In case 5 , the endothelial cell density decreased from 2117 cells $/ \mathrm{mm}^{2}$ at 6 months to 954 cells $/ \mathrm{mm}^{2}$ at 12 months post-operatively. The central corneal thickness was $517 \pm 3 \mu \mathrm{m}$ (range, $468-561 \mu \mathrm{m}$; Table 3 ). The mean thickness of the posterior shelf was $175 \pm 8 \mu \mathrm{m}$ (range, 168-185 $\mu \mathrm{m}$; Fig. 6).

\section{TABLE 1. Demographic Preoperative Data}

\begin{tabular}{|c|c|c|c|c|c|}
\hline $\begin{array}{l}\text { No., Age (y), } \\
\text { Sex, OD(S) }\end{array}$ & Indication for Surgery & $\begin{array}{l}\text { Concomitant } \\
\text { Disease }\end{array}$ & BCVA & Refraction & $\begin{array}{c}\text { Topographic } \\
\text { Astigmatism (D) }\end{array}$ \\
\hline 1,47 , male, OS & $\begin{array}{l}\text { Pre-descemet dystrophy } \\
\text { X-linked ichtychosis }\end{array}$ & - & $20 / 30$ & +1.25-1.0@73 & 0.6 \\
\hline 2,42, male, OS & $\begin{array}{l}\text { Pre-descemet dystrophy } \\
\text { X-linked ichtychosis }\end{array}$ & - & $20 / 60$ & +4.50-1.50@85 & 1.0 \\
\hline 3,75 , male, OD & PBK & ARMD & $\mathrm{HM}$ & Plano & NA \\
\hline 4,72 , female, OD & PBK & $\begin{array}{l}\text { Cellophane } \\
\text { maculopathy }\end{array}$ & $20 / 60$ & +1.0 & 1.6 \\
\hline 5,75, female, OS & PBK & Amblyopia & $20 / 100$ & -2.25 & NA \\
\hline
\end{tabular}

PBK, pseudophakic bullous keratopathy; ARMD, age-related macular degeneration; HM, hand movement; NA, not available. 
TABLE 2. Postoperative Data at 12-month Follow-up

\begin{tabular}{|c|c|c|c|c|c|}
\hline No. & BCVA & Refraction & $\begin{array}{c}\text { Topographic } \\
\text { Astigmatism (D) }\end{array}$ & $\begin{array}{l}\text { Most Recent Postoperative } \\
\text { ECD (cells } / \mathrm{mm}^{2} \text { ) }\end{array}$ & $\begin{array}{c}\text { Sutures all out/ } \\
\text { partly out }\end{array}$ \\
\hline 1 & $20 / 20^{*}$ & $+1.25-5.0 @ 175$ & 5.1 & 2237 & All, $19^{\#}$ \\
\hline 2 & $20 / 25$ & $+5.0-2.75 @ 25$ & 2.1 & 1897 & All, $21^{\#}$ \\
\hline 3 & $20 / 60$ & +4.0-5.5@82 & 5.6 & 1856 & Partly out \\
\hline 4 & $20 / 30$ & +3.75-2.0@120 & 0.6 & 2015 & Partly out \\
\hline 5 & $20 / 40$ & $-1.75-0.75 @ 65$ & 2.9 & 954 & Partly out \\
\hline
\end{tabular}

ECD, endothelial cell density.

*Best contact lens-corrected visual acuity.

\#Number of weeks after keratoplasty.

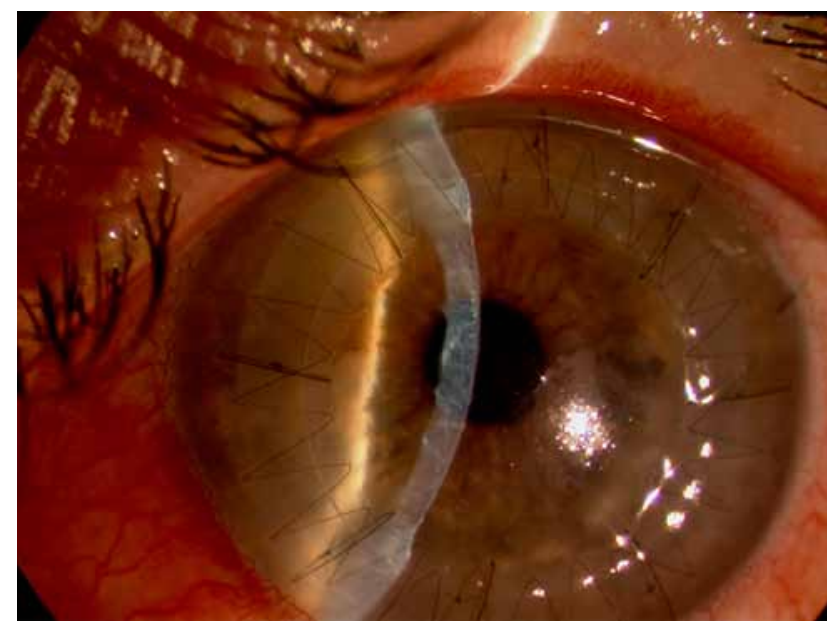

FIGURE 3. Case 2, left eye. Slit-lamp photograph after femtosecond laser-assisted inverted mushroom keratoplasty. At 1 week postoperatively, an increased stromal edema centrally and at the peripheral interface was noted.

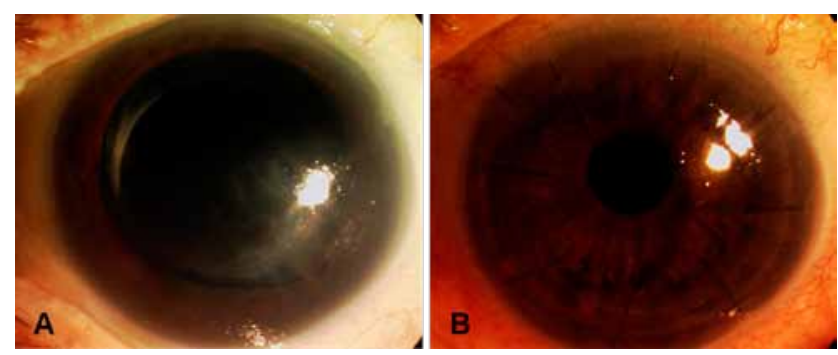

FIGURE 4. Case 5, left eye. Slit-lamp photographs after femtosecond laser-assisted inverted mushroom keratoplasty. A, Preoperatively, a pseudophakic bullous keratopathy was seen. B, One month postoperatively, the cornea is clear, and an excellent fit between the graft and recipient was noted. 

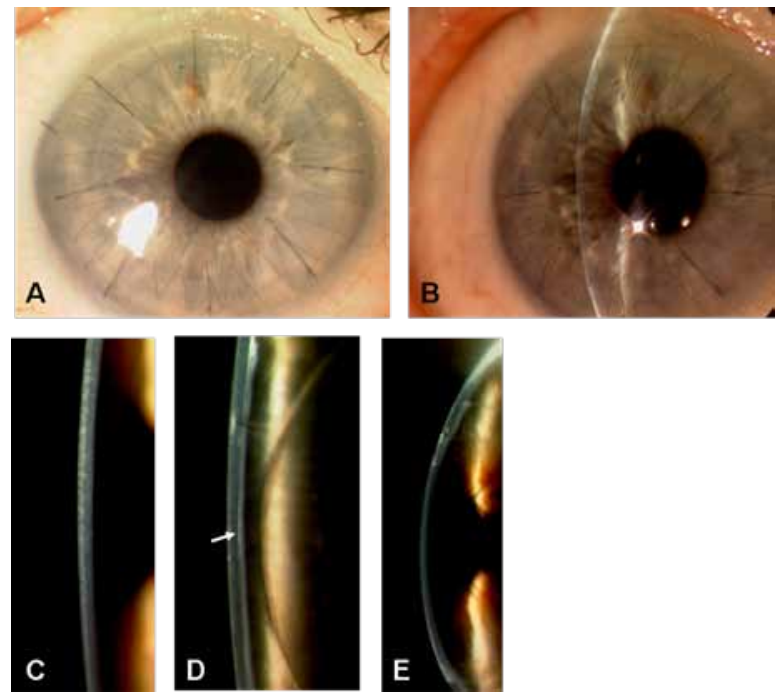

FIGURE 5. Case 4, right eye. Slitlamp photographs at 3 months postoperatively. The cornea is centrally clear ( $A-C)$, and the adhesion between the graft and the recipient at the peripheral lamellar interface is excellent ( $D$ and $E)$. Note the higher reflectivity of the interface (arrow).

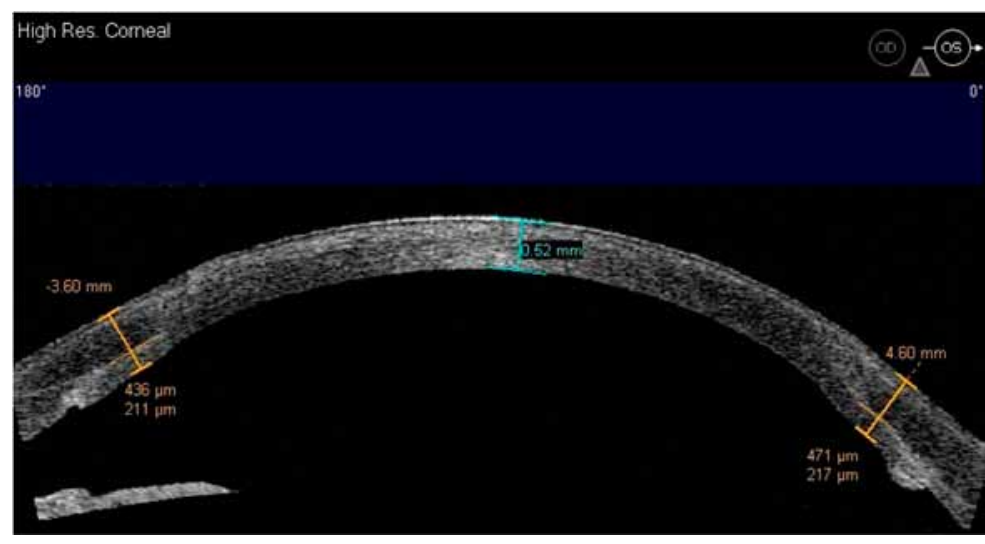

FIGURE 6. Case 1, left eye. Optical coherence tomography image after femtosecond laser-assisted inverted mushroom keratoplasty. This figure shows the top-hat configuration in the $180^{\circ}-0^{\circ}$ meridian. The central corneal thickness is $520 \mu \mathrm{m}$, and the posterior shelf varies from 211 to $217 \mu \mathrm{m}$. 


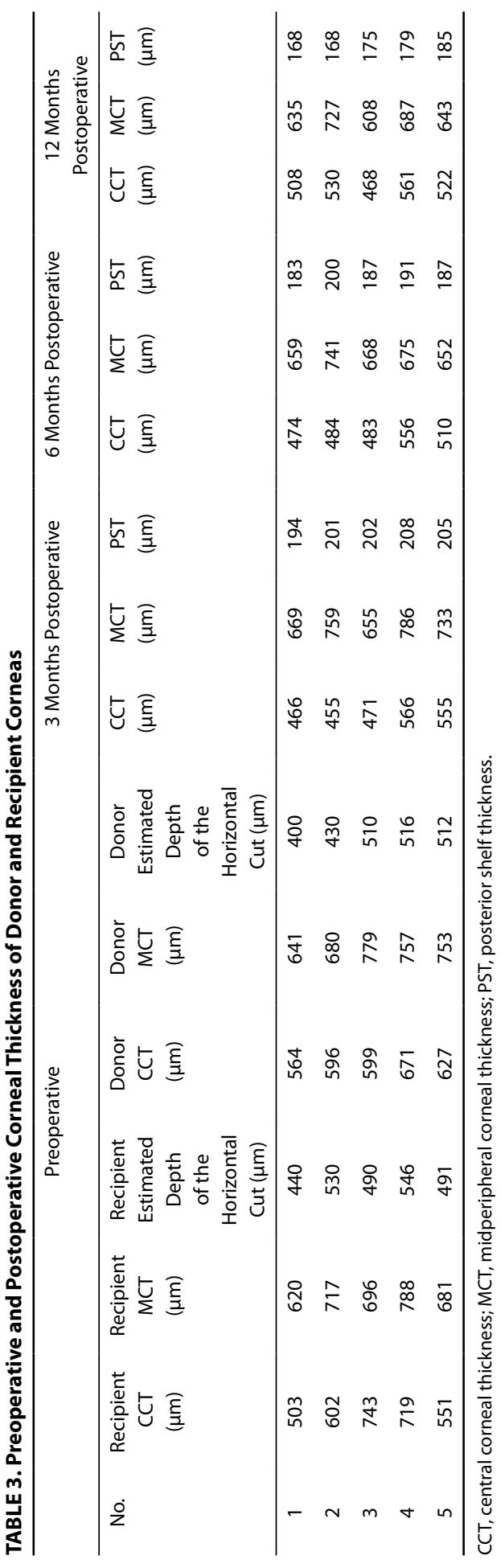




\section{DISCUSSION}

In conventional penetrating keratoplasty, surgeons are still faced with problems related to insufficient healing such as unstable refraction and wound dehiscence after the sutures have been removed. Recently, new lamellar transplantation techniques such as deep lamellar endothelial keratoplasty and Descemet-stripping automated endothelial keratoplasty in corneal transplantation surgery may result in a better stabilization of the anterior surface of the cornea. ${ }^{12-16}$ The deep donor-recipient lamellar interface in deep lamellar endothelial keratoplasty and Descemet-stripping automated endothelial keratoplasty may reduce the potential visual acuity because of optical interference, and interface haze has been a major reason for the low acceptance of lamellar transplantation techniques among corneal surgeons. ${ }^{17}$

To combine the advantages of a clear optical center of a penetrating graft and the increased wound healing of a lamellar graft, a mixed technique of a full-thickness graft with a peripheral lamellar wound has been advocated. For this purpose, Busin ${ }^{11}$ and Busin and $\mathrm{Arffa}^{18}$ have popularized a mushroom configuration by using a mechanical trephination technique. In a case report of a patient with a deep stromal scar, a microkeratome-assisted mushroom technique was used consisting of a large 9.0-mm anterior stromal lamella and a small $5.0-\mathrm{mm}$ posterior button. ${ }^{18}$ This resulted in minimal removal of recipient endothelium with fast postoperative healing and preservation of most of the recipient endothelium. Complete suture removal was performed 3 months after surgery. Best spectacle-corrected visual acuity improved from $20 / 60$ to $20 / 20$ at 6 months postoperatively, and refraction was $-2.50-1.00 \times 20^{\circ}$. In another study of 8 eyes, Busin ${ }^{11}$ showed that an inverted mushroom lamellar dissection technique for creating a top-hat configuration allowed complete suture removal by 3 months postoperatively. Refractive astigmatism before and after suture removal was minimized to $4 \mathrm{D}$ or less in all eyes. In addition, because the 7.0-mm anterior surface of the donor button is smaller than the 9.0-mm posterior one, more endothelial cells can be transplanted, thereby prolonging the longevity of the graft.

Ideally, the optimal penetrating keratoplasty procedure would consist of a custommade nonmechanical trephination that allows the formation of a self-sealing donor/ recipient apposition. Seitz et $\mathrm{al}^{19}$ were the first to report in a laboratory study the feasibility of an industrial femtosecond laser source to create an inverse mushroom shape in polymethylmethacrylate blocks and porcine corneas. In porcine eyes, light microscopy displayed trephination edges delineated by partly confluent gas bubbles with tissue bridges in between. Electron microscopy showed normal adjacent collagen fibers. ${ }^{19}$ Another laboratory study has shown a reduction in wound leakage of a top-hat configuration compared with a standard penetrating keratoplasty configuration..$^{20,21}$ 
Recently, the femtosecond laser was effectively used for preparing the posterior corneal disc in endothelial transplantation techniques in an in vitro study ${ }^{22}$ and in a patient. ${ }^{23}$ We coined the term femtosecond Descemet-stripping endothelial keratoplasty (FS-DSEK) for this procedure. ${ }^{23}$ This study showed that a femtosecond laser with programmable software and hardware configurations can create custom-made (inverse) mushroom corneal grafts. The BCVA was $20 / 40$ or better in 4 of 5 cases and limited in 3 cases by amblyopia and macular disease. Best potential macular acuity (pinhole acuity) was $20 / 50$ in case $3,20 / 30$ in case 4 , and $20 / 40$ in case 5 . The mean refractive astigmatism at 12 months was $-3.20 \pm 2.0 \mathrm{D}$, and 2 of 5 patients had $>3 \mathrm{D}$ of refractive astigmatism. Buratto and Bohm ${ }^{24}$ used the femtosecond laser for penetrating keratoplasty in a series of 7 cases. At 3 months, all grafts were clear with good endothelial cell counts and normal corneal thickness in each case. High levels of astigmatism after suture removal may still be a problem, even after femtosecond laser-inverted mushroom keratoplasty as seen in our study in case 1 . The creation of orientation teeth by the femtosecond laser, which has been described before with other lasers, may result in better astigmatism management. ${ }^{25}$ The endothelial cell density was adequate in 4 cases after 12 months and decreased in 1 patient from 2117 cells $/ \mathrm{mm}^{2}$ at 6 months to 954 cells $/ \mathrm{mm}^{2}$ at 12 months postoperatively. We noticed no early rejection period that might be responsible for the endothelial cell loss. It is unclear whether the long-term endothelial cell loss after femtosecond laser-assisted inverted mushroom keratoplasty will be the same as in penetrating keratoplasty, where an endothelial cell loss as high as $53 \%$ has been shown 3 years after transplantation. ${ }^{26}$ The measured thickness of the posterior shelf was in accordance with the preoperatively estimated thickness (175 vs. $200 \mu \mathrm{m}$ ). However, an exact match for the horizontal lamellar plane in graft and host cornea is difficult to obtain when the preoperative thicknesses of both corneas are different because of a different hydration state. A disparity of the graft/host anterior stromal cornea above the lamellar plane could result in a suboptimal fit of the graft into the host wound bed and higher levels of astigmatism.

Three patients complained of photophobia during the first postoperative month that resolved after increasing the topical steroid treatment. It was suspected that the photophobia resulted from the high levels of energy (up to $7.0 \mu \mathrm{J}$ ) with the 15-kHz laser used for making the posterior outer vertical side cut. Recently, studies showed that cutting a laser in situ keratomileusis flap with a 15-kHz laser caused stromal cell death and inflammatory cell infiltration that decreased after the introduction of the 30 - and $60-\mathrm{kHz}$ laser. ${ }^{27,28} \mathrm{~A}$ similar mechanism may apply for lamellar cuts made deeper in the cornea during femtosecond laser-assisted keratoplasty. Modifications of the femtosecond laser in energy levels (down to $3.0 \mu \mathrm{J}$ ) and firing rate with the introduction of a femtosecond $60-\mathrm{kHz}$ laser for performing femtosecond laser-enabled keratoplasty will result in a presumably lower incidence of photophobia. A decreased incidence of the transient light 
sensitivity syndrome has also been seen after femtosecond laser energy reduction in the course of cutting the laser in situ keratomileusis flap. 29,30

Fast wound healing was shown in 2 cases where all sutures were removed at 19 (case 1 ) and 21 weeks (case 2 ) after surgery. In the other 3 cases, the sutures were only partially removed because of the satisfactory level of astigmatism.

Femtosecond laser-assisted penetrating keratoplasty techniques will revolutionize corneal transplant surgery because cutting techniques and dimensions may be individualized. In the near future, eye banks will be able to produce custom-designed corneal grafts on the basis of the specifications of a corneal surgeon. The laser preparation of the donor tissue is automated and thereby reduces the technical difficulties and risks involved with the preoperative manual dissection of an (inverse) mushroom graft. This will allow eye banks to control the quality of grafts after the femtosecond laser procedure, thereby enhancing quality management of corneal transplantation surgery in general. 


\section{REFERENCES}

1. Zirm E. Eine erfolgreiche totale Keratoplastik. Albrecht Von Graefes Arch Ophthalmol. 1906;64:580593.

2. Williams KA, Muehlberg SM, Lewis RF, et al. How successful is corneal transplantation? A report from the Australian Corneal Graft Register. Eye. 1995;9:219-227.

3. Williams KA, Esterman AJ, Bartlett C, et al. How effective is penetrating corneal transplantation? Factors influencing long-term outcome in multivariate analysis. Transplantation. 2006;81:896-901.

4. Nagra PK, Hammersmith KM, Rapuano CJ, et al.Wound dehiscence after penetrating keratoplasty. Cornea. 2006;25:132-135.

5. Elder MJ, Stack RR. Globe rupture following penetrating keratoplasty: how often, why, and what can we do to prevent it? Cornea. 2004;23:776-780.

6. Vajpayee RB, Sharma N, Sinha R, et al. Laser in-situ keratomileusis after penetrating keratoplasty. Surv Ophthalmol. 2003;48:503-514.

7. Nuijts RM, Abhilakh Missier KA, Nabar VA, et al. Artisan toric lens implantation for correction of postkeratoplasty astigmatism. Ophthalmology. 2004;111:1086-1094.

8. Seitz B, Langenbucher A, Kus MM, et al. Nonmechanical corneal trephination with the excimer laser improves outcome after penetrating keratoplasty. Ophthalmology. 1999;106:1156-1164.

9. Abou-Jaoude ES, Brooks M, Katz DG, et al. Spontaneous wound dehiscence after removal of single continuous penetrating keratoplasty suture. Ophthalmology. 2002;109:1291-1296.

10. Mannis MJ, Holland EJ, Beck RW, et al. Clinical profile and early surgical complications in the Cornea Donor Study. Cornea. 2006;25:164-170.

11. Busin M. A new lamellar wound configuration for penetrating keratoplasty surgery. Arch Ophthalmol. 2003;121:260-265.

12. Melles GR, Lander F, Beekhuis WH, et al. Posterior lamellar keratoplasty for a case of pseudophakic bullous keratopathy. Am J Ophthalmol. 1999;127:340-341.

13. Terry MA, Ousley PJ. Deep lamellar endothelial keratoplasty in the first United States patients: early clinical results. Cornea. 2001;20:239-243.

14. Gorovoy MS. Descemet-stripping automated endothelial keratoplasty. Cornea. 2006;25:886-889.

15. Price FW Jr, Price MO. Descemet's stripping with endothelial keratoplasty in 200 eyes: early challenges and techniques to enhance donor adherence. J Cataract Refract Surg. 2006;32:411-418.

16. Koenig SB, Covert DJ. Early results of small-incision Descemet's stripping and automated endothelial keratoplasty. Ophthalmology. 2007;114:221-226.

17. Rich LF. Expanding the scope of lamellar keratoplasty. Trans Am Ophthalmol Soc. 1999;97:771-814.

18. Busin M, Arffa RC. Microkeratome-assisted mushroom keratoplasTy with minimal endothelial replacement. Am J Ophthalmol. 2005;140:138-140.

19. Seitz B, Brunner H, Viestenz A, et al. Inverse mushroom-shaped nonmechanical penetrating keratoplasty using a femtosecond laser. Am J Ophthalmol. 2005;139:941-944.

20. Ignacio TS, Nguyen TB, Chuck RS, et al. Top hat wound configuration for penetrating keratoplasty using the femtosecond laser: a laboratory model. Cornea. 2006;25:336-340.

21. Steinert RF, Ignacio TS, Sarayba MA."Top hat"-shaped penetrating keratoplasty using the femtosecond laser. Am J Ophthalmol. 2007;143:689-691.

22. Seitz B, Langenbucher A, Hofmann-Rummelt C, et al. Nonmechanical posterior lamellar keratoplasty using the femtosecond laser (femto-plak) for corneal endothelial decompensation. Am J Ophthalmol. 2003;136:769-772. 
23. Cheng YY, Pels E, Nuijts RM. Femtosecond-laser-assisted Descemet's stripping endothelial keratoplasty. J Cataract Refract Surg. 2007;33:152-155.

24. Buratto $L$, Bohm E. The use of the femtosecond laser in penetrating keratoplasty. Am J Ophthalmol. 2007;143:737-742.

25. Behrens A, Seitz B, Kuchle M, et al. “Orientation teeth" in nonmechanical laser corneal trephination for penetrating keratoplasty: 2.94 micron Er:YAG v 193 nm ArF excimer laser. Br J Ophthalmol. 1999;83:1008-1012.

26. Patel SV, Hodge DO, Bourne WM. Corneal endothelium and postoperative outcomes 15 years after penetrating keratoplasty. Am J Ophthalmol. 2005;139:311-319.

27. Kim JY, Kim MJ, Kim TI, et al. A femtosecond laser creates a stronger flap than a mechanical microkeratome. Invest Ophthalmol Vis Sci. 2006; 47:599-604.

28. Netto MV, Mohan RR, Medeiros FW, et al. Femtosecond laser and microkeratome corneal flaps: comparison of stromal wound healing and inflammation. J Refract Surg. 2007;23:667-676.

29. Stonecipher KG, Dishler JG, Ignacio TS, et al. Transient light sensitivity after femtosecond laser flap creation: clinical findings and management. J Cataract Refract Surg. 2006;32:91-94.

30. Hu MY, McCulley JP, Cavanagh HD, et al. Comparison of the corneal response to laser in situ keratomileusis with flap creation using the FS15 and FS30 femtosecond lasers: clinical and confocal microscopy findings. J Cataract Refract Surg. 2007;33:673-681. 



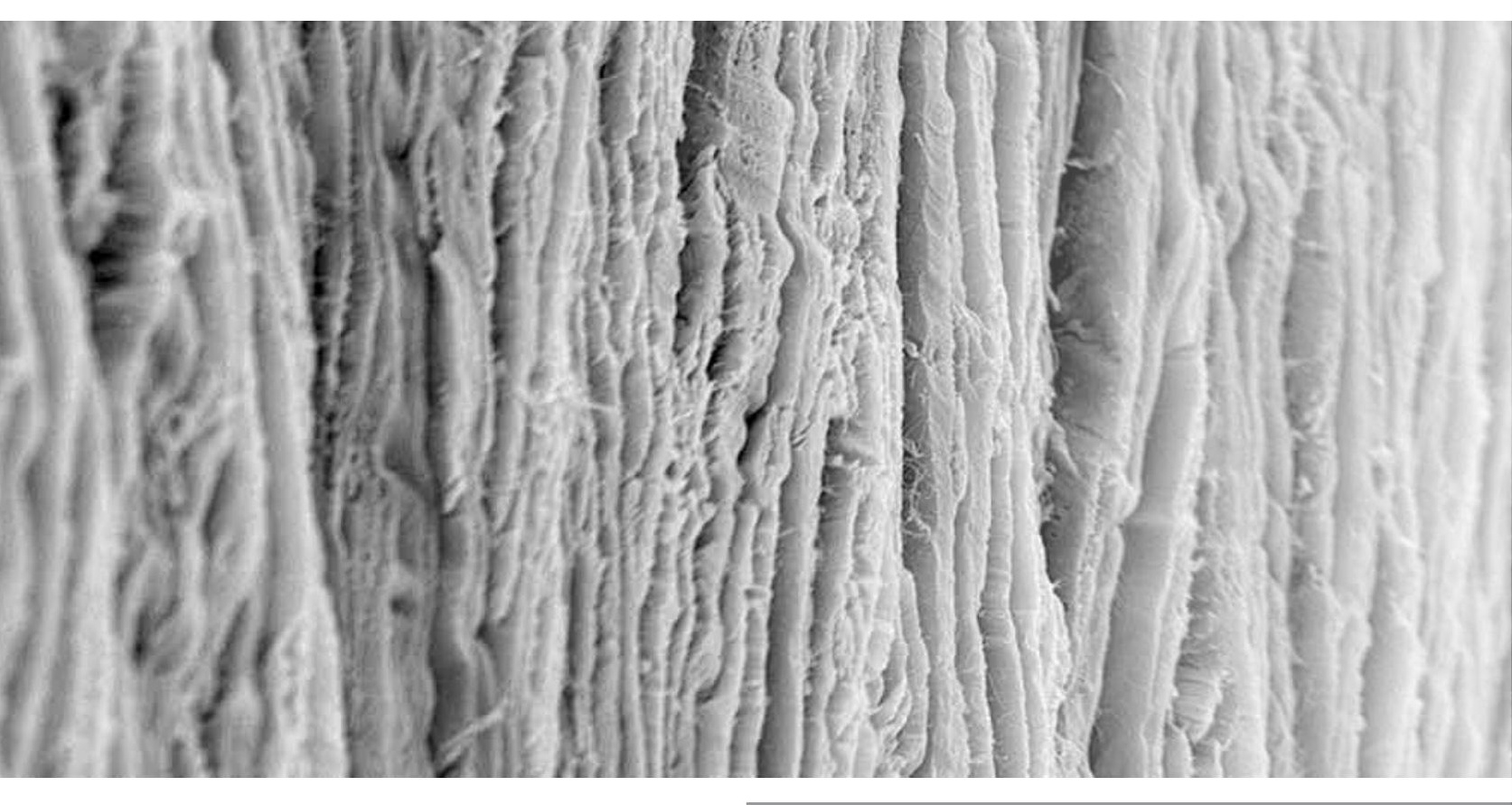




\title{
CHAPTER 10
}

Endothelial Cell Loss and Visual Outcome of Deep Anterior

Lamellar Keratoplasty versus

Penetrating Keratoplasty: A

Randomized Multicenter Clinical

Trial

\author{
Yanny Y. Y. Cheng \\ Nienke Visser \\ Jan S. Schouten \\ Robert-Jan Wijdh \\ Elisabeth Pels \\ Hugo van Cleynenbreugel \\ Catharina A. Eggink \\ Michel J. W. Zaal \\ Wilhelmina J. Rijneveld \\ Rudy M.M.A. Nuijts
}

Ophthalmology. 2011 Feb;118(2):302-9 


\section{ABSTRACT}

Objective: To compare endothelial cell (EC) loss, visual and refractive outcomes, and complications after deep anterior lamellar keratoplasty (DALK) and penetrating keratoplasty (PK).

Design: Randomized, multicenter clinical trial.

Participants: Fifty-six eyes of 56 patients with a corneal stromal pathology not affecting the endothelium were randomized to DALK or PK.

Methods: The DALK procedure was performed according to Anwar's big-bubble technique. Patients underwent an ophthalmic examination preoperatively and 3, 6, and 12 months postoperatively.

Main Outcome Measures: Endothelial cell loss, refractive and topographic astigmatism, spherical equivalent, uncorrected visual acuity, and best spectacle-corrected visual acuity (BSCVA) were measured, and complications were recorded.

Results: Endothelial cell loss was significantly higher after PK compared with DALK procedures performed without perforation of Descemet's membrane (12 months: $27.7 \%$ $\pm 11.1 \%$ vs. $12.9 \% \pm 17.6 \%$ ). The BSCVA was significantly better in the PK group at 3 and 6 months after surgery but was not significantly different 12 months after surgery $(0.39$ \pm 0.3 logarithm of the minimum angle of resolution [logMAR] in DALK and $0.31 \pm 0.3$ logMAR in PK). At 12 months postoperatively, refractive and topographic astigmatism in the DALK and PK groups were $-3.37 \pm 2.3$ diopters $(D)$ and $-3.76 \pm 2.1 \mathrm{D}(P=0.53)$, and $3.57 \pm 2.3 \mathrm{D}$ and $4.16 \pm 2.0 \mathrm{D}(P=0.34)$, respectively. (Micro)perforation of the Descemet's membrane occurred in $32 \%(9 / 28)$ of the DALK eyes, and $18 \%(5 / 28)$ of the patients required conversion to PK. Endothelial cell loss was not significantly different between DALK and PK when cases with perforation of Descemet's membrane were included in the (intention-to-treat) analysis (12 months: $19.1 \pm 21.6$ vs. $27.7 \pm 11.1 P=0.112$ ). Rejection episodes were reported in 1 patient in the DALK group (epithelial rejection) and 3 patients in the PK group (all endothelial rejections). No graft failure occurred.

Conclusions: One year after DALK performed without perforation of Descemet's membrane, EC loss is significantly lower, whereas the BSCVA is comparable to that in the PK group. In addition, no endothelial rejection occurred in the DALK group. However, Descemet's membrane perforation remains a major complication in DALK and warrants improvements to standardize the big-bubble technique. 


\section{INTRODUCTION}

Corneal stromal pathologies, such as keratoconus, keratitis sequelae, and stromal dystrophies, account for more than one third of the indications for keratoplasty. ${ }^{1}$ Penetrating keratoplasty (PK) has been shown to be a safe technique, achieving good visual outcomes in these patients. However, graft failure remains a problem and has been reported in $18 \%$ to $34 \%$ of the cases. ${ }^{2,3}$ The major reasons for graft failure are endothelial rejection and endothelial failure, which account for more than $50 \%$ of graft failures. ${ }^{4}$ After PK, endothelial cell (EC) counts have been shown to decrease approximately $70 \%$ in 10 years, having a major impact on long-term graft survival. ${ }^{4}$

Although the concept of lamellar grafting was first introduced more than 150 years ago, lamellar transplantation techniques that only transplant the anterior side of the cornea underwent a recent revival in popularity. In deep anterior lamellar keratoplasty (DALK), the corneal stroma is removed down to the Descemet's membrane or a thin layer of stroma is left on top of the Descemet's membrane. The corneal stroma is replaced with a lamellar donor button, preserving the recipient's Descemet's membrane and endothelium. This technique can be performed in patients with corneal stroma pathologies not affecting the endothelium. A main advantage of DALK is prevention of long-term EC loss. In addition, because the endothelium (which is the major target for rejection) is not grafted, the incidence of immunologic rejection is reduced. Other theoretic advantages of DALK include shorter postoperative steroid therapy and a reduced risk of anterior synechiae or intraocular infection due to a "closed eye" procedure.

Many countries have a shortage in corneas suitable for donation. Major reasons for discarding donor corneas are abnormalities of the anterior corneal stroma and a decreased vitality of the corneal endothelium. Approximately one third of the corneas are unsuitable for PK because of a low endothelial cell density (ECD) $\left(<2000\right.$ cells $/ \mathrm{mm}^{2}$ in most cornea banks). ${ }^{1,5}$ Shortage of donor tissue has resulted in a considerable waiting time for corneal transplantation in many countries, including The Netherlands. Previously discarded corneas because of abnormalities of the endothelium may be used in DALK, reducing the waiting lists of eye banks.

Visual outcomes of DALK might be limited compared with PK if baring of Descemet's membrane is incomplete and interface haze occurs. Deep dissection at the level of the Descemet's membrane is thought to lead to better visual outcomes because of the absence of stromal scarring at the graft- host stromal interface. ${ }^{6,7}$ Various techniques have been used to accomplish baring of the Descemet's membrane, including intrastromal air injection, ${ }^{8,9}$ hydrodelamination, ${ }^{6}$ viscoelastic dissection using an-air-to-endothelium interface, ${ }^{10}$ and blunt spatula delamination. ${ }^{11}$ Anwar and Teichmann ${ }^{12}$ introduced the "big-bubble" technique, in which partial thickness trephination is followed by stromal air injection to form an air bubble to dissect the Descemet's membrane from the posterior 
stroma. This facilitates a safer exposure of Descemet's membrane and produces a smooth surface for high-quality vision. Several interventional studies using this technique showed promising results regarding visual outcome and graft survival. ${ }^{13-15}$ However, this big-bubble technique is technically challenging and may result in perforations of Descemet's membrane in up to $57 \%$ of the cases. ${ }^{16}$ No randomized controlled studies have been performed to compare the effect of the Anwar's big-bubble DALK technique with PK. The Dutch Lamellar Corneal Transplantation Study has been designed to compare EC loss, visual and refractive outcomes, and complication profile after DALK and PK.

\section{MATERIALS AND METHODS}

This randomized multicenter trial (Dutch Lamellar Corneal Transplantation Study) was conducted at 5 centers in The Netherlands. The institutional review boards of all participating centers approved the study. Informed consent was obtained from all patients. The patients were recruited between April 2005 and February 2008. Since December 11, 2006, the trial was registered at the Dutch trial register as the Dutch Lamellar Corneal Transplantation Study (http://www.trialregister.nl, no. ISRCTN02191620) and performed in accordance to the principles of the Declaration of Helsinki.

Inclusion criteria were patients with keratoconus intolerant for contact lens wear (without prior hydrops or Descemet's rupture) and stromal opacifications not reaching Descemet's membrane and without concomitant endothelial disease. Exclusion criteria were best-corrected visual acuity $\geq 20 / 50$, keratoconus with previous hydrops or Descemet's rupture, or corneal stromal opacification reaching Descemet's membrane.

The medical history was recorded, and all patients underwent a comprehensive ophthalmic examination. Preoperative data included patient age, gender, refractive error, intraocular pressure (IOP), preoperative lens status, and ocular comorbidities. All donor corneas were obtained from the same Cornea Bank in The Netherlands (Cornea Bank Amsterdam). The method to measure the ECDs of the donor tissue was described by Pels and Schuchard. ${ }^{17}$

\section{Surgical procedures}

The selection criteria of the donor cornea for the DALK and PK group were the same. The surgical procedure in the DALK group was performed according to Anwar's big-bubble technique. ${ }^{12}$ The recipient cornea was trephined for approximately $60 \%$ of its thickness using a 7.75- or 8.0-mm Hessburg-Barron vacuum trephine. A partial-thickness anterior keratectomy was then performed. A 30-gauge needle, attached to a 1-to 3-ml air-filled syringe was brought into the remaining stroma in a paracentral position. A bubble was formed by pressing the air-filled syringe. A 15-degree knife was used to make an incision 
in the anterior wall of the big bubble. A blunt spatula was introduced in the space left by the collapsed bubble and used to form a dissection plane that was subsequently filled with a cohesive viscoelastic. The stromal layers were divided in 4 quadrants and excised with blunt-tipped microscissors. After removal of the Descemet's membrane and endothelium, the corneal donor button was trephined with a $8.0-$ or $8.25-\mathrm{mm}$ Hessburg-Barron trephine and sutured into the bed using 8 interrupted 10-0 nylon sutures and a continuous 11-0 nylon suture. Suture removal was based on the level of refractive astigmatism and guided by topographic astigmatism patterns in both groups.

In the PK group, the recipient cornea was trephined using a 7.75- or 8.0-mm HessburgBarron vacuum trephine, and the donor cornea was trephined with a 8.0 - or $8.25-\mathrm{mm}$ Hessburg-Barron trephine. In all cases a combined suturing technique of 8 interrupted 10-0 nylon sutures with a continuous 11-0 nylon suture was used. Selective suture removal was based on the topographic astigmatism pattern.

Postoperatively, all patients in the DALK and PK groups received the same topical schedule. Topical dexamethasone $0.1 \%$ drops (Ratiopharm, Zaandam, The Netherlands) were tapered after surgery with the following schedule: 6 times daily for 1 month, 4 times daily for 1 month, 3 times daily for 1 month, 2 times daily for 3 months, 1 time daily for 6 months, and thereafter 1 time daily on alternate days until all sutures were removed. Topical chloramphenicol $0.5 \%$ drops (Ratiopharm) were given 3 times daily for the first 3 months and then stopped.

\section{Outcome measures}

The main outcome measure is the amount of EC loss. Endothelial cell densities are measured using the Noncon Robo SP 8000 (Konan, Hyogo, Japan). Three specular photographs were taken, and each image was analyzed by selecting 50 cells in the center of the image to reduce sampling error. Secondary outcome measures included uncorrected visual acuity (UCVA), best spectacle-corrected visual acuity (BSCVA), manifest refraction, refractive astigmatism, topographic astigmatism (EyeMap EH-290, Alcon, Fort Worth, TX), and intra- and post-operative complications. The UCVA and BSCVA were determined using the Early Treatment of Diabetic Retinopathy Study letter charts and converted to logarithm of the minimum angle of resolution (logMAR) measurements. Vision levels of counting fingers, hand movements, light perception, and no light perception were substituted by logMAR values of 1.7, 2.0, 2.5, and 3.0, respectively. All outcome parameters were recorded preoperatively and at 3,6 , and 12 months after surgery.

\section{Sample size}

The sample size was calculated on the basis of an EC loss of $8 \%$ after DALK and $45 \%$ after PK in 1 year, based on our preliminary experiences and reported amounts of EC 
loss in previous studies. ${ }^{18-20}$ By assuming an a of 0.05 , a power of $90 \%$, and $10 \%$ loss to follow-up, 28 patients were required in each treatment group.

\section{Randomization and blinding}

Consecutive patients in each center were randomly assigned to DALK or PK treatment. The randomization code was generated using a permuted block size of 2 . The assigned treatment plans were then sent to the surgeon.

\section{Statistical analysis}

Intention-to-treat analysis was performed for all outcomes measures, and additional analysis was performed for the EC loss without perforation of the Descemet's membrane. Data were described as mean \pm standard deviation for continuous variables and as individual counts and percentages for categoric variables. Continuous data were analyzed using the Student $t$ test for differences between groups. The chi-square or Fisher exact test was used to compare categoric data. Comparisons of preoperative data and postoperative data within a group were performed using a linear regression model. A $P$ value of less than 0.05 was considered to be statistically significant. Statistical analysis was performed using SPSS for Windows (version 15.0, SPSS Inc., Chicago, IL).

\section{RESULTS}

\section{Participant flow}

The patient flow is displayed in Figure 1 (available at http://aaojournal.org). Fifty-six eyes of 56 patients were included, with 28 patients in both the DALK and PK groups. Five patients in the DALK group did not receive the allocated treatment and were converted to a PK because of Descemet's membrane perforations.

All patients in the PK group received the allocated treatment. All 28 patients in the DALK group completed the 3-month postoperative follow-up visit. One patient was lost to follow-up 6 months postoperatively. This was a young patient who was satisfied with the postoperative result and refused to attend further follow-up visits. In the PK group, all patients completed the follow-up visits.

\section{Patient characteristics}

Patient characteristics are listed in Table 1 (available at http://aaojournal.org). The mean age in the DALK group was not significantly different from the mean age in the PK group $(43.4 \pm 15.9$ years and $42.9 \pm 14.1$ years, respectively; $P=0.899)$. Twenty-six patients in the DALK group and 25 patients in the PK group were phakic, which was not significantly different. In addition, 6 patients ( 3 in the DALK group, 3 in the PK group) were diagnosed 


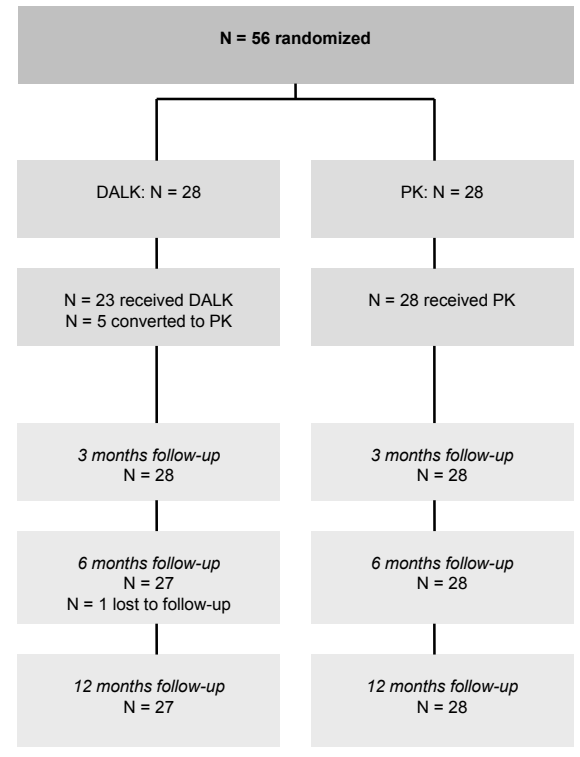

Figure 1. Participant Flow

DALK = deep anterior lamellar keratoplasty; $\mathrm{PK}=$ penetrating keratoplasty; $\mathrm{N}=$ number of patients

TABLE 1. Preoperative Patient Characteristics

DALK PK $\quad$ Pvalue

Eyes $(\mathrm{N})$

Age in years (mean $\pm S D)$

Women (N, \%)

\section{Diagnosis}

\section{Keratoconus}

Herpes simplex virus keratitis

Corneal ulcer

Pre-Descemet dystrophy

Groenouw stromal dystrophy

\section{Recipient lens status}

Phakic

$26(92.9 \%)$

$2(7.1 \%)$

$25(89.0 \%)$

Pseudophakic

$\begin{array}{ll}28 & 28 \\ 43.4 \pm 15.9 & 42.9 \pm 14.1 \\ 13(46 \%) & 13(46 \%)\end{array}$

$15(53.6 \%)$

$15(53.6 \%)$

7 (25.0\%)

$9(32.1 \%)$

$6(21.4 \%)$

$1(3.6 \%)$

0

$2(7.1 \%)$

1 (3.6\%)

$\mathrm{DALK}=$ deep anterior lamellar keratoplasty; $\mathrm{PK}=$ penetrating keratoplasty; $\mathrm{N}=$ number of eyes; $\mathrm{SD}=$ standard deviation 
with cataract. In these patients, cataract extraction was performed at least 12 months after keratoplasty. No patients underwent primary cataract surgery or a combined procedure.

Keratoconus and corneal scarring caused by herpes simplex virus keratitis were the most important reasons for keratoplasty in both the DALK and PK groups. In the DALK group, 6 patients required a keratoplasty for a corneal ulcer, compared with 1 patient in the PK group. Three patients in the PK group required a keratoplasty for a corneal dystrophy.

\section{Endothelial cell density}

The ECDs for both groups are shown in Table 2. Specular photographs of sufficient quality could not be obtained in all patients in the DALK group because of stromal scarring (preoperatively) or microfolds of the interface (postoperatively). In the intention-to-treat analysis, the preoperative ECDs were significantly higher in the PK group ( $P=0.045)$. Post-operative ECDs did not differ significantly between the DALK and PK groups. The EC loss was determined for all patients in the DALK and PK groups with available preoperative and postoperative ECDs. The EC loss was comparable between the 2 groups at all follow-up visits.

The analysis without perforation of the Descemet's membrane showed that the EC loss in the PK group was significantly higher at all follow-up visits compared with the DALK group. The EC loss continued in both groups but was significantly higher in the PK

TABLE 2. Preoperative/Donor and Postoperative Endothelial Cell Loss of Deep Anterior Lamellar Keratoplasty and Penetrating Keratoplasty

\begin{tabular}{|c|c|c|c|c|c|}
\hline & \multicolumn{2}{|c|}{ DALK } & \multicolumn{2}{|c|}{ PK } & \multirow[t]{2}{*}{$P$ value } \\
\hline & Mean $\pm S D(N)$ & Range & Mean \pm SD $(\mathrm{N})$ & Range & \\
\hline \multicolumn{6}{|c|}{$\begin{array}{l}\text { Endothelial cell density } \\
\left(\text { cells } / \mathrm{mm}^{2} \text { ) }\right.\end{array}$} \\
\hline Preoperative & $2489 \pm 318(22)$ & $1820-2855$ & $2712 \pm 109(28)$ & $2500-2900$ & 0.045 \\
\hline $3 \mathrm{mos}$ & $2018 \pm 643^{\ddagger}(20)$ & $449-2725$ & $2111 \pm 301^{\ddagger}(28)$ & $1654-2930$ & 0.547 \\
\hline $6 \mathrm{mos}$ & $1831 \pm 647^{\ddagger}(24)$ & $441-2635$ & $2095 \pm 297^{\ddagger}(28)$ & $1683-2882$ & 0.097 \\
\hline \multirow[t]{2}{*}{$12 \mathrm{mos}$} & $1936 \pm 643^{\ddagger}(24)$ & $576-3000$ & $1966 \pm 321^{\ddagger}(28)$ & $1256-2427$ & 0.849 \\
\hline & $P=0.038^{\dagger}$ & & $P<0.01^{\dagger}$ & & \\
\hline \multicolumn{6}{|c|}{ Endothelial cell loss (\%) s,\# $^{\text {s,\# }}$} \\
\hline $3 \mathrm{mos}$ & $16.1 \pm 24.5(20)$ & $-9.3 \pm 79.2$ & $22.4 \pm 9.8(28)$ & $-8.5 \pm 36.4$ & 0.375 \\
\hline $6 \mathrm{mos}$ & $18.5 \pm 24.3(22)$ & $3.7 \pm 79.6$ & $22.5 \pm 10.9(28)$ & $-6.8 \pm 36.1$ & 0.599 \\
\hline $12 \mathrm{mos}$ & $19.1 \pm 21.6(22)$ & $12.2 \pm 73.3$ & $27.7 \pm 11.1(28)$ & $12.4 \pm 55.1$ & 0.112 \\
\hline
\end{tabular}


TABLE 2. Preoperative/Donor and Postoperative Endothelial Cell Loss of Deep Anterior Lamellar Keratoplasty and Penetrating Keratoplasty (continued)

\begin{tabular}{lccccc}
\hline & \multicolumn{2}{c}{ DALK } & \multicolumn{2}{c}{ PK } & $P^{*}$ value $^{*}$ \\
\cline { 2 - 6 } & Mean \pm SD (N) & Range & Mean \pm SD (N) & Range \\
\hline Endothelial cell loss (\%) s, $^{2}$ & & & & & \\
3 mos & $6.6 \pm 17.1(17)$ & -9.3 to 42.5 & $22.4 \pm 9.8(28)$ & -8.5 to 36.4 & 0.003 \\
6 mos & $9.9 \pm 16.8(18)$ & -3.7 to 43.4 & $22.5 \pm 10.9(28)$ & -6.8 to 36.1 & 0.024 \\
12 mos & $12.9 \pm 17.6(18)$ & -12.2 to 42.2 & $27.7 \pm 11.1(28)$ & $12.4-55.1$ & 0.007 \\
\hline
\end{tabular}

DALK = deep anterior lamellar keratoplasty; $\mathrm{N}=$ number of eyes; $\mathrm{PK}=$ penetrating keratoplasty; $\mathrm{SD}=$ standard deviation.

${ }^{*} P$ value between the DALK and PK groups ( $t$ test).

${ }^{\dagger} P$ value between preoperative and postoperative data within a group (linear regression model).

${ }^{\ddagger} P<0.05$ between a specific postoperative visit and the preoperative visit (linear regression model).

${ }^{5}$ Endothelial cell loss is determined in patients with available preoperative and postoperative ECDs (paired data).

"Based on intention-to-treat analysis.

"Based on analysis without perforation of the Descemet's membrane.

group and reached $27.7 \% \pm 11.1 \%$ in the PK group and $12.9 \% \pm 17.6 \%$ in the DALK group $(P=0.007)$ at 12 months postoperatively.

\section{Visual outcomes}

Visual outcomes are shown in Table 3. The preoperative UCVA in the DALK and PK groups was comparable. After surgery, there was a significant improvement in UCVA in both groups. Three months postoperatively, the UCVA was significantly better in the PK group, compared with the DALK group $(0.72 \pm 0.4 \log M A R$ and $0.95 \pm 0.4 \log M A R$, respectively; $P=0.021)$. However, the UCVA in the DALK group continued to improve and was comparable to that in the PK group at both 6 and 12 months postoperatively.

The BSCVA was comparable in the DALK and PK groups before surgery. After surgery, there was a significant improvement in BSCVA in both groups. Three and 6 months postoperatively, the BSCVA was significantly better in the PK group compared with the DALK group. However, the BSCVA continued to improve in the DALK group and was not significantly different from the PK group 12 months postoperatively $(0.39 \pm 0.3 \operatorname{logMAR}$ and $0.31 \pm 0.3 \log M A R$, respectively; $P=0.368$ ). In addition, the BSCVA gain in the DALK and $P K$ groups was comparable. One year after surgery, the BSCVA gain was 0.52 logMAR in the DALK group and 0.50 logMAR in the PK group $(P=0.897)$.

In the PK group there were 4 eyes at 3 months, 5 eyes at 6 months, and 5 eyes at 12 months with a BSCVA of logMAR $\leq 0(\geq 20 / 20)$. In the DALK group there were 2 eyes with a BSCVA of logMAR $\leq 0(\geq 20 / 20)$ at 3,6 , and 12 months postoperatively. 


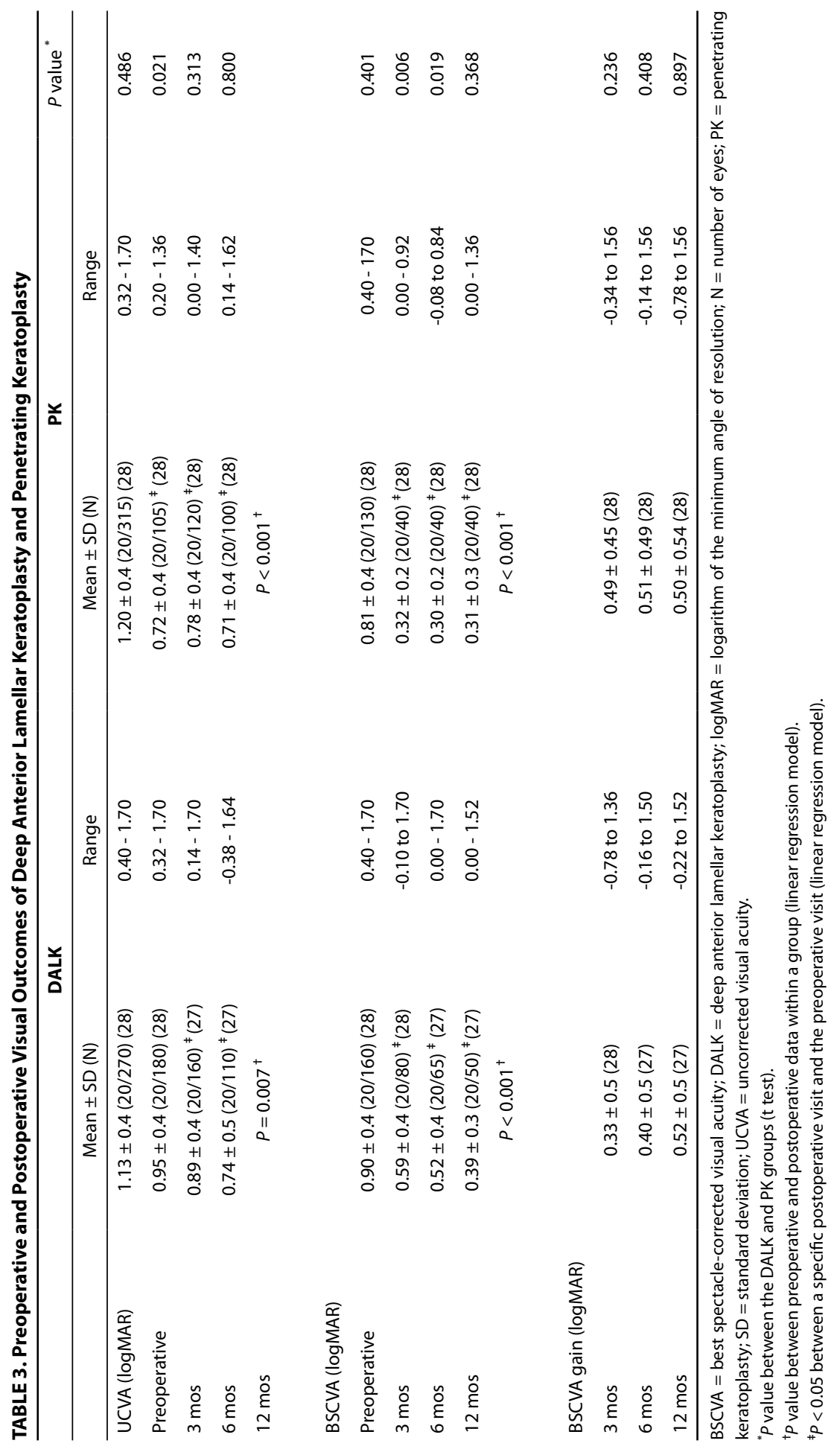




\section{Refractive outcomes}

Refractive outcomes are shown in Table 4. Preoperatively and at all postoperative followup visits, the spherical equivalent, refractive astigmatism, and topographic astigmatism in the DALK and PK groups were not significantly different.

There was a trend toward less myopia after DALK and PK; however, this was not significant. Refractive astigmatism was significantly increased in the PK group at 3 and 12 months after surgery, compared with the preoperative situation. Topographic astigmatism was significantly reduced after surgery at all follow-up visits in the PK group and at 12 months follow-up in the DALK group, compared with preoperatively.

TABLE 4. Preoperative and Postoperative Refractive Outcomes of Deep Anterior Lamellar Keratoplasty and Penetrating Keratoplasty

\begin{tabular}{|c|c|c|c|c|c|}
\hline & \multicolumn{2}{|c|}{ DALK } & \multicolumn{2}{|c|}{ PK } & \multirow[t]{2}{*}{$P$ value } \\
\hline & Mean \pm SD $(\mathrm{N})$ & Range & Mean $\pm S D(N)$ & Range & \\
\hline \multicolumn{6}{|c|}{ Spherical equivalent (D) } \\
\hline Preoperative & $-3.39 \pm 6.7(28)$ & -17.00 to 7.38 & $-3.71 \pm 5.3(28)$ & -15.50 to 3.75 & 0.851 \\
\hline $3 \mathrm{mos}$ & $-2.68 \pm 3.2(28)$ & -14.63 to 2.38 & $-1.53 \pm 3.2(28)$ & -13.25 to 2.50 & 0.201 \\
\hline $6 \mathrm{mos}$ & $-2.88 \pm 3.6(27)$ & -13.50 to 2.50 & $-2.14 \pm 3.5(28)$ & -11.00 to 3.63 & 0.450 \\
\hline \multirow[t]{2}{*}{$12 \mathrm{mos}$} & $-2.02 \pm 3.0(27)$ & -10.25 to 1.63 & $-2.30 \pm 3.8(28)$ & -12.50 to 2.00 & 0.774 \\
\hline & $P=0.738^{\dagger}$ & & $P=0.278^{\dagger}$ & & \\
\hline \multicolumn{6}{|c|}{ Refractive astigmatism (D) } \\
\hline Preoperative & $-2.63 \pm 2.9(28)$ & -11.0 to 0.0 & $-2.26 \pm 3.3(28)$ & -11.0 to 0.0 & 0.667 \\
\hline $3 \mathrm{mos}$ & $-4.11 \pm 2.6(28)$ & -12.0 to 0.0 & $-4.17 \pm 1.7^{\ddagger}(28)$ & -8.0 to -1.0 & 0.918 \\
\hline $6 \mathrm{mos}$ & $-3.88 \pm 2.8(27)$ & -12.0 to 0.0 & $-3.33 \pm 1.9(28)$ & -7.0 to -0.5 & 0.415 \\
\hline \multirow[t]{2}{*}{$12 \mathrm{mos}$} & $-3.37 \pm 2.3(27)$ & -9.5 to 0.0 & $-3.76 \pm 2.1^{\ddagger}(28)$ & -8.0 to -0.2 & 0.526 \\
\hline & $P=0.167^{\dagger}$ & & $P=0.030^{\dagger}$ & & \\
\hline
\end{tabular}

Topographic astigmatism

(D)

$\begin{array}{lccccc}\text { Preoperative } & 6.61 \pm 4.9(23) & 1.50-17.51 & 6.90 \pm 6.4(22) & 0.60-26.60 & 0.863 \\ 3 \mathrm{mos} & 5.64 \pm 3.0(28) & 0.43-16.83 & 4.70 \pm 2.7^{\ddagger}(28) & 0.60-9.08 & 0.250 \\ 6 \mathrm{mos} & 4.74 \pm 3.2(27) & 0.83-13.27 & 3.77 \pm 2.4^{\ddagger}(28) & 0.60-10.25 & 0.232 \\ 12 \mathrm{mos} & 3.57 \pm 2.3^{\ddagger}(27) & 0.40-9.72 & 4.16 \pm 2.0^{\ddagger}(28) & 1.01-8.98 & 0.337 \\ & P=0.023^{\dagger} & & P=0.026^{\dagger} & \end{array}$

$\mathrm{D}=$ diopters; $\mathrm{DALK}=$ deep anterior lamellar keratoplasty; $\mathrm{N}=$ number of eyes; $\mathrm{PK}=$ penetrating keratoplasty; $\mathrm{SD}=$ standard deviation.

${ }^{*} P$ value between the DALK and PK groups ( ${ }^{t}$ test).

${ }^{\dagger} P$ value between preoperative and postoperative data within a group (linear regression model).

${ }^{\ddagger} P<0.05$ between a specific postoperative visit and the preoperative visit (linear regression model). 
A refractive astigmatism of more than 3 diopters (D) was present preoperatively in $44 \%$ of the DALK group and $32 \%$ of the PK group $(P=0.263)$. Postoperatively, this percentage did not differ significantly between the 2 groups either (12 months: $58 \%$ of the DALK group vs. $64 \%$ of the PK group, $P=0.455)$. A topographic astigmatism of more than $3 \mathrm{D}$ was present in $70 \%$ of the DALK group and $73 \%$ of the PK group before surgery $(P=0.538)$. After surgery, there was a trend toward a decreased number of patients with more than $3 \mathrm{D}$ topographic astigmatism in the DALK group compared with the PK group, but this did not reach statistical significance (12 months: $50 \%$ vs. $76 \%$, respectively, $P=$ 0.055).

\section{Intraoperative and postoperative complications}

Complications are shown in Table 5 (available at http://aaojournal.org). No statistical tests were performed because of sparse data. The big bubble could be achieved in 19 eyes (82.6\%), and in 4 eyes (17.4\%) manual dissection was performed. Intraoperative perforation of the Descemet's membrane occurred in 9 of 28 patients (32\%), and 5 of these patients (18\%) required conversion to a PK. In the remaining 4 patients, the perforation was managed preoperatively $(n=3)$ with injection of air in the anterior chamber or after a postoperative air injection in the anterior chamber $(n=1)$. Six of 9 patients with a perforation had stromal scarring caused by herpes simplex virus keratitis, 2 patients had keratoconus, and 1 patient had a corneal ulcer.

Suture reactions occurred in 8 of 23 patients in the DALK group (35\%) and 7 of 33 patients in the PK group (21\%). At 12 months follow-up, complete suture removal had been performed in $22 \%$ of the patients in the DALK group and $20 \%$ of the patients in the PK group. No wound dehiscence occurred.

TABLE 5. Complications of Deep Anterior Lamellar Keratoplasty and Penetrating Keratoplasty

\begin{tabular}{lcc}
\hline Complication & DALK & PK \\
& N (\%) & \\
\hline Microperforation Descemet membrane & & - \\
Conversion to PK & $9(32 \%)$ & - \\
Suture reactions & $5(18 \%)$ & $7(21 \%)$ \\
Wound dehiscence & $8(35 \%)$ & 0 \\
Elevated intraocular pressure & 0 & $3(9 \%)$ \\
Cataract & $5(22 \%)$ & 0 \\
Rejection episodes & 0 & $3(9 \%)$ \\
Graft failure & $1(4 \%)$ & 0 \\
\end{tabular}

DALK = deep anterior lamellar keratoplasty; $\mathrm{PK}=$ penetrating keratoplasty; $\mathrm{N}=$ number of patients 
During the 12-month follow-up, an elevated IOP, defined as an IOP greater than 21 $\mathrm{mmHg}$, was recorded in 5 patients (22\%) in the DALK group and 3 patients (9\%) in the PK group. All patients in both the DALK and PK groups were still using topical steroid treatment after 12 months of follow-up.

A rejection episode occurred in 1 patient in the DALK group and 3 patients in the PK group. The patient in the DALK group was diagnosed with epithelial rejection, and the 3 patients in the PK group were diagnosed with endothelial rejection. All 4 patients were treated with steroids, and no graft failure occurred.

\section{DISCUSSION}

This randomized multicenter trial compares EC loss, visual and refractive outcomes, and complication profile after DALK and PK during a follow-up of 12 months. We found a lower EC loss, comparable UCVA and BSCVA, comparable refractive outcomes, and no endothelial rejections in the DALK group compared with the PK group. Perforation of Descemet's membrane occurred in $32 \%$ of the patients, and $18 \%$ of the patients required conversion to a PK. These results are in accordance with a previous randomized clinical trial comparing DALK and PK. ${ }^{21}$

Preoperative ECDs were significantly lower in DALK eyes than PK eyes. Six patients in the DALK group required a keratoplasty for a corneal ulcer, and all 6 patients previously had severe keratouveitis with hypopyon formation. A few studies have suggested that severe anterior segment inflammation might lead to a decreased preoperative ECD. ${ }^{22,23}$ An additional explanation might be that in vitro ECDs in donor corneas are not entirely comparable to in vivo measurements. Moreover, the selection of donor cornea is also based on ECD with a minimum of 2300 cells $/ \mathrm{mm}^{2}$.

In the intention-to-treat analysis, the EC loss was comparable between the DALK and PK groups during all postoperative follow-up visits. However, the EC loss in patients without microperforations of the Descemet's membrane was significantly higher in the PK group compared with the DALK group. A sufficiently high ECD is one of the major factors contributing to long-term graft survival. The higher EC loss in PK-treated eyes is in accordance with previous studies. ${ }^{18,19,21,24-26} \mathrm{~A}$ large cohort study with a 15-year follow-up showed that the ECD continues to decrease after a PK, until it stabilizes approximately 10 years after surgery. ${ }^{4}$ At this time, total EC loss is estimated at $70 \%$.

Three months after DALK surgery, EC loss was $6.6 \%$, compared with the preoperative situation. Surgically induced EC loss in DALK-treated eyes has been reported to be $10 \%{ }^{22}$ or approximately 400 cells $/ \mathrm{mm}^{2}{ }^{27}$ This is in accordance with our results $\left(\sim 400\right.$ cells $/ \mathrm{mm}^{2}$ between the preoperative visit and 3-month follow-up). After 1-year follow-up, EC loss in the DALK group was $12.9 \%$. Previous studies show an EC loss ranging from $6 \%$ to $20 \%$ 
in the first year after DALK surgery. ${ }^{15,22,24,28}$ Shimazaki et al $^{21}$ performed a randomized controlled trial comparing DALK and PK and found a stabilization of EC loss 6 months after a DALK procedure. In addition, van Dooren et a ${ }^{27}$ described an initial decrease in ECDs of approximately $11 \%$ in 6 months, followed by a physiologic rate of cell loss. However, we found no evidence for EC loss stabilization within 12 months of a DALK procedure. A longer follow-up is needed to evaluate this further.

Initial postoperative visual outcomes in our study were significantly better in the PK group compared with the DALK group. However, 6 to 12 months after surgery, both the UCVA and BSCVA in the DALK group reached the same level as in the PK group. This indicates that Anwar's big-bubble technique achieves a deep dissection up to the level of the Descemet's membrane and does not lead to substantial interface hazing. Earlier stabilization of visual outcomes after a PK, compared with a DALK, has also been suggested by a previous study. ${ }^{28}$ Although a significantly better best-corrected visual acuity (BCVA) after DALK has been reported, ${ }^{16}$ the majority of the studies report similar values in DALK eyes compared with PK patients..$^{18,21,24,25,28-31}$ One study reported better BCVA in PK patients, but this might be due to incomplete stromal removal in DALK eyes. ${ }^{32}$

In our study, refractive astigmatism values after DALK were comparable to PK with an average refractive astigmatism of -3.37 and $-3.76 \mathrm{D}$, respectively, after 12 months followup. This is in comparison with previous studies. ${ }^{24,30,33}$ However, in our study only approximately $20 \%$ of the patients in both the DALK and PK groups had all sutures removed. Because suture removal is likely to influence astigmatism values, a longer follow-up is needed to evaluate final astigmatism values after suture removal. One study, in which sutures had been removed in all patients in both the DALK and PK groups, reported comparable median refractive astigmatism values of $-4.00 \mathrm{D}$ (range -5.00 to $-2.00 \mathrm{D}$ ) in the DALK group and $-3.25 \mathrm{D}$ (range -4.50 to $-2.25 \mathrm{D}$ ) in the PK group after a follow-up of 28 months. $^{30}$

The high preoperative topographic astigmatism values in both the DALK and PK eyes (6.61 $\pm 4.9 \mathrm{D}$ and $6.90 \pm 6.4 \mathrm{D}$, respectively) may be caused by corneal irregularities due to epithelial irregularities, corneal scars, or keratoconus. Postoperative topographic astigmatism in the DALK group did not differ from the PK group. However, the influence of suture removal is unknown. A randomized study with all sutures in situ did not find a significant difference in topographic astigmatism either after a follow-up of 6 months. ${ }^{21} \mathrm{~A}$ different study evaluated keratometric astigmatism values 1 month after suture removal (4 months for DALK and 1 year for PK) and reported a lower degree of astigmatism in DALK eyes. ${ }^{19}$ On the other hand, Ardjomand et al $^{7}$ did not find a significant difference in astigmatism after suture removal.

The main complication of a DALK procedure is intraoperative perforation of the Descemet's membrane. Studies using comparable deep-dissection techniques report a perforation rate ranging from $6 \%$ to $57 \% .^{12,14}-16,24,26,31$ However, the reported perforation 
rates also depend on whether discrimination is made between microperforations and larger perforations that require a conversion. We report a total perforation (microperforations and larger perforations) rate of $32 \%$ and $18 \%$ of the patients required conversion to PK. This might be partially explained by a learning curve of the surgeon. An additional explanation is that 6 of 9 patients with Descemet's membrane perforations in our study had been diagnosed with severe stromal scarring caused by herpes simplex virus keratitis. Al-Torbak et $\mathrm{al}^{34}$ showed that the perforation rate is significantly higher in eyes with deep corneal scars. They hypothesize that scarring leads to tight junction formation between deep stromal lamellae and the Descemet's membrane, which causes perforation during deep dissection. More research is needed to evaluate whether a PK should be preferred over a DALK in eyes with severe scarring in the posterior stroma. However, we advise caution when using the big-bubble technique in eyes with stromal scarring.

Preservation of healthy endothelium in DALK procedures has several advantages. Earlier discontinuation of topical steroids and consequently a reduced incidence of glaucoma and cataract have been suggested. ${ }^{21}$ However, because all patients in our study were still using topical steroids 1 year after surgery, we did not expect to find a lower incidence of IOP elevation in DALK-treated eyes. An additional advantage of endothelium preservation is a reduced incidence of immunologic rejection. Endothelial rejection, one of the major reasons for graft failure, occurred in 3 patients in the PK group, but did not occur in any patient in the DALK group in this study. Epithelial rejection did occur in 1 patient in the DALK group in our study. Both epithelial and stromal rejections have been reported incidentally in DALK eyes, but affected corneas generally make a good recovery once the inflammatory response is controlled. ${ }^{26,32,35}$

In conclusion, DALK procedures performed without perforation of Descemet's membrane resulted in a significantly lower EC loss, while at the same time achieving equally good visual outcomes as a PK procedure. However, in the occurrence of intraoperative perforation of Descemet's membrane, this advantage is lost. Because complete suture removal was only performed in the minority of DALK and PK eyes, a final comparison in post-operative visual outcomes, astigmatism differences, and suture-related problems will only be possible after all sutures have been removed. At this moment, the relatively high incidence of Descemet's membrane perforation in DALK warrants the presence of a donor cornea with good endothelial vitality because the donor cornea has to be suitable for a PK in case of conversion. Further surgical improvements are needed to standardize the big-bubble technique to reduce the perforation rate. 


\section{REFERENCES}

1. Muraine M, Toubeau D, Gueudry J, Brasseur G. Impact of new lamellar techniques of keratoplasty on eye bank activity. Graefes Arch Clin Exp Ophthalmol 2007;245:32- 8.

2. Beckingsale $\mathrm{P}$, Mavrikakis I, Al-Yousuf $\mathrm{N}$, et al. Penetrating keratoplasty: outcomes from a corneal unit compared to national data. Br J Ophthalmol 2006;90:728 -31.

3. Thompson RW Jr, Price MO, Bowers PJ, Price FW Jr. Longterm graft survival after penetrating keratoplasty. Ophthalmology 2003;110:1396-402.

4. Patel SV, Hodge DO, Bourne WM. Corneal endothelium and postoperative outcomes 15 years after penetrating keratoplasty. Am J Ophthalmol 2005;139:311-9.

5. Armitage WJ, Easty DL. Factors influencing the suitability of organ-cultured corneas for transplantation. Invest Ophthalmol Vis Sci 1997;38:16-24.

6. Sugita J, Kondo J. Deep lamellar keratoplasty with complete removal of pathological stroma for vision improvement. Br J Ophthalmol 1997;81:184-8.

7. Ardjomand N, Hau S, McAlister JC, et al. Quality of vision and graft thickness in deep anterior lamellar and penetrating corneal allografts. Am J Ophthalmol 2007;143:228 -35.

8. Archila EA. Deep lamellar keratoplasty dissection of host tissue with intrastromal air injection. Cornea 1984;3:217-8.

9. Chau GK, Dilly SA, Sheard CE, Rostron CK. Deep lamellar keratoplasty on air with lyophilised tissue. Br J Ophthalmol 1992;76:646 -50.

10. Melles GR, Lander F, Rietveld FJ, et al. A new surgical technique for deep stromal, anterior lamellar keratoplasty. Br J Ophthalmol 1999;83:327-33.

11. Anwar M, Teichmann KD. Deep lamellar keratoplasty: surgical techniques for anterior lamellar keratoplasty with and without baring of Descemet's membrane. Cornea 2002;21: 374-83.

12. Anwar M, Teichmann KD. Big-bubble technique to bare Descemet's membrane in anterior lamellar keratoplasty. J Cataract Refract Surg 2002;28:398-403.

13. Vajpayee RB, Tyagi J, Sharma N, et al. Deep anterior lamellar keratoplasty by big-bubble technique for treatment corneal stromal opacities. Am J Ophthalmol 2007;143:954 -7.

14. Fogla R, Padmanabhan P. Results of deep lamellar keratoplasty using the big-bubble technique in patients with keratoconus. Am J Ophthalmol 2006;141:254 -9.

15. Fontana L, Parente G, Tassinari G. Clinical outcomes after deep anterior lamellar keratoplasty using the big-bubble technique in patients with keratoconus. Am J Ophthalmol 2007; 143:117-24.

16. Anshu A, Parthasarathy A, Mehta JS, et al. Outcomes of therapeutic deep lamellar keratoplasty and penetrating keratoplasty for advanced infectious keratitis: a comparative study. Ophthalmology 2009;116:615-23.

17. Pels E, Schuchard Y. Organ-culture preservation of human corneas. Doc Ophthalmol 1983;56:14753.

18. Panda A, Bageshwar LM, Ray M, et al. Deep lamellar keratoplasty versus penetrating keratoplasty for corneal lesions. Cornea 1999;18:172-5.

19. Trimarchi F, Poppi E, Klersy C, Piacentini C. Deep lamellar keratoplasty. Ophthalmologica 2001;215:389-93.

20. Ing JJ, Ing HH, Nelson LR, et al. Ten-year postoperative results of penetrating keratoplasty. Ophthalmology 1998;105:1855-65.

21. Shimazaki J, Shimmura S, Ishioka M, Tsubota K. Randomized clinical trial of deep lamellar keratoplasty vs penetrating keratoplasty. Am J Ophthalmol 2002;134:159-65. 
22. Morris E, Kirwan JF, Sujatha S, Rostron CK. Corneal endothelial specular microscopy following deep lamellar keratoplasty with lyophilised tissue. Eye (Lond) 1998;12:619-22.

23. Reijo A, Antti V, Jukka M. Endothelial cell loss in herpes zoster keratouveitis. Br J Ophthalmol 1983;67:751- 4 .

24. Bahar I, Kaiserman I, Srinivasan S, et al. Comparison of three different techniques of corneal transplantation for keratoconus. Am J Ophthalmol 2008;146:905-12.

25. Krumeich JH, Knulle A, Krumeich BM. Deep anterior lamellar (DALK) vs. penetrating keratoplasty (PKP): a clinical and statistical analysis [in German]. Klin Monbl Augenheilkd 2008;225:637-48.

26. Borderie VM, Werthel AL, Touzeau O, et al. Comparison of techniques used for removing the recipient stroma in anterior lamellar keratoplasty. Arch Ophthalmol 2008;126:31-7.

27. van Dooren BT, Mulder PG, Nieuwendaal CP, et al. Endothelial cell density after deep anterior lamellar keratoplasty (Melles technique). Am J Ophthalmol 2004;137:397-400.

28. Kawashima M, Kawakita T, Den S, et al. Comparison of deep lamellar keratoplasty and penetrating keratoplasty for lattice and macular corneal dystrophies. Am J Ophthalmol 2006;142: 304-9.

29. Silva CA, Schweitzer de Oliveira E, de Sena Junior MP, Barbosa de Sousa L. Contrast sensitivity in deep anterior lamellar keratoplasty versus penetrating keratoplasty. Clinics (Sao Paulo) 2007;62:705-8.

30. Watson SL, Ramsay A, Dart JK, et al. Comparison of deep lamellar keratoplasty and penetrating keratoplasty in patients with keratoconus. Ophthalmology 2004;111:1676-82.

31. Han DC, Mehta JS, Por YM, et al. Comparison of outcomes of lamellar keratoplasty and penetrating keratoplasty in keratoconus. Am J Ophthalmol 2009;148:744 -51.

32. Funnell CL, Ball J, Noble BA. Comparative cohort study of the outcomes of deep lamellar keratoplasty and penetrating keratoplasty for keratoconus. Eye (Lond) 2006;20:527-32.

33. Low U, Kohlhof JK, Ruprecht KW, Hille K. Development of visual acuity and refraction in deep lamellar and penetrating keratoplasty (KPL) [in German]. Klin Monbl Augenheilkd 2006;223:289 -93.

34. Al-Torbak AA, Al-Motowa S, Al-Assiri A, et al. Deep anterior lamellar keratoplasty for keratoconus. Cornea 2006;25:408-12.

35. Noble BA, Agrawal A, Collins C, et al. Deep anterior lamellar keratoplasty (DALK): visual outcome and complications for a heterogeneous group of corneal pathologies. Cornea 2007;26: 59-64. 


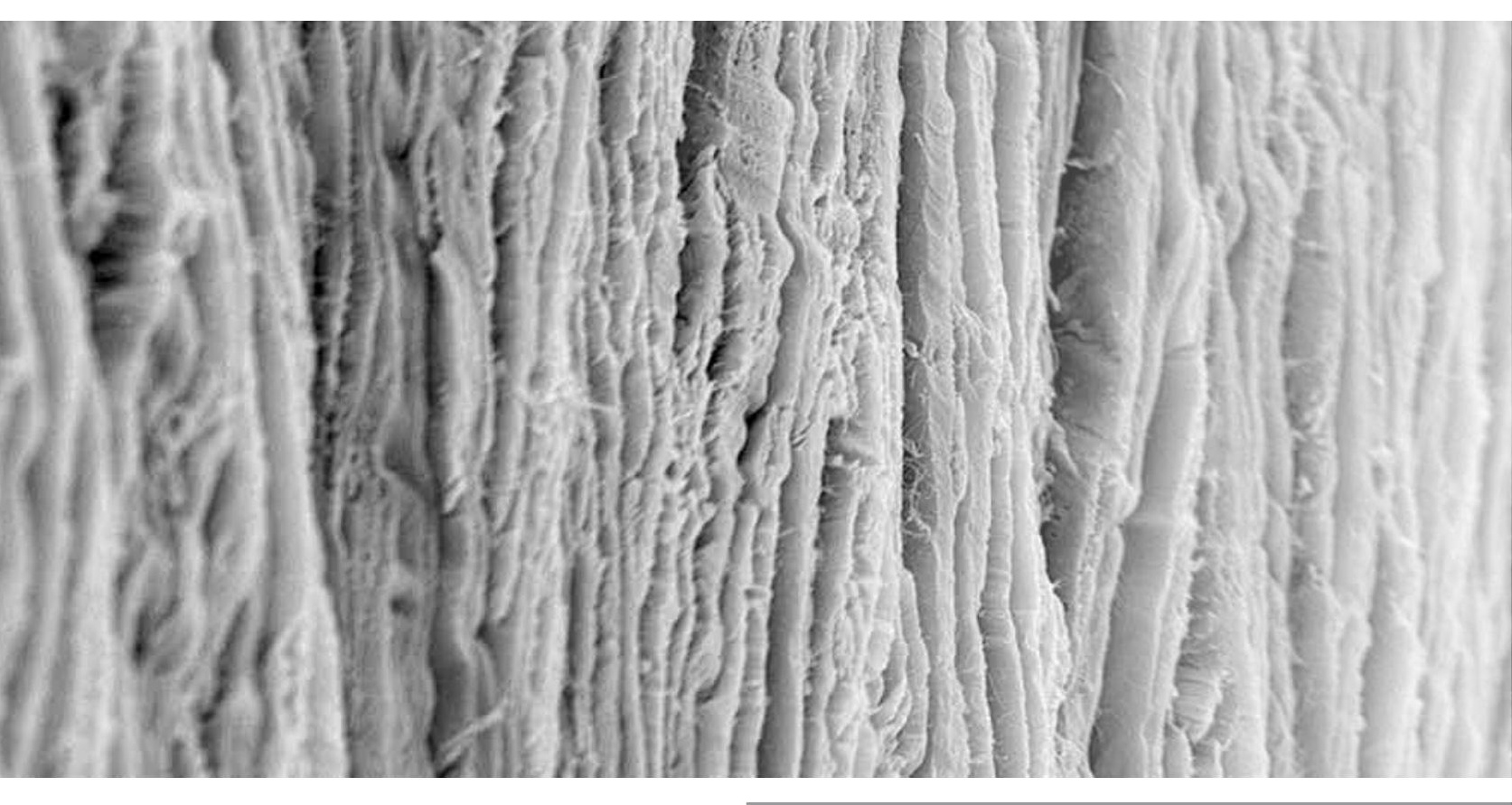




\section{CHAPTER 11}

Quality of Vision after Deep

Anterior Lamellar Keratoplasty

and Penetrating Keratoplasty: A

Randomized Multicenter Clinical

Trial

Yanny Y.Y. Cheng

Tom J.T.P. van den Berg

Robert-Jan Wijdh

Catharina A. Eggink

Wilhelmina J. Rijneveld

Rudy M.M.A. Nuijts

Submitted 


\section{ABSTRACT}

Aim: To compare the quality of vision after deep anterior lamellar keratoplasty (DALK) using the big-bubble technique and penetrating keratoplasty (PK).

Methods: Fifty-six eyes of 56 patients with a corneal stromal pathology and healthy endothelium were included and were randomized to DALK or PK. The surgical procedure in the DALK group was performed according to Anwar's big-bubble technique. Patients underwent an extensive ophthalmic examination prior to surgery and 3, 6 and 12 months postoperatively.

Results: Straylight was not significantly different after DALK compared to PK at 12 months follow-up (1.34 \pm 0.3 logarithm of straylight vs $1.38 \pm 0.3$ logarithm of straylight, $P=0.642$ ). During the 12 months follow-up, there was a significant improvement in straylight and contrast sensitivity after DALK and PK. The change in straylight was significantly correlated with the change of best spectacle-corrected visual acuity (BSCVA) in the DALK, but not in the PK group. The change in contrast sensitivity correlated significantly with the change of BSCVA in both groups.

Conclusions: Straylight and contrast sensitivity showed a significant improvement after both DALK and PK, whereas BSCVA was comparable between groups. Our results show that the quality of vision, measured by straylight, contrast sensitivity, and visual acuity, after DALK is comparable with that attained after PK. 


\section{INTRODUCTION}

Penetrating keratoplasty (PK) has been the standard treatment for diseases of the corneal stroma that not affect the endothelium, such as keratoconus, infectious keratitis and stromal keratitis. ${ }^{1}$ PK has been shown to be a safe technique, achieving good visual outcomes. However, graft failure remains a problem, and is primarily caused by endothelial rejection and endothelial failure which account for more than $50 \%$ of graft failures. $^{2}$

In recent years, deep anterior lamellar keratoplasty (DALK) has become increasingly popular. The major advantage of DALK is that the host's healthy endothelium can be preserved, which avoids the risk of immunologic rejection against the endothelium. Furthermore, long-term endothelial cell loss is lower following DALK as compared to PK. ${ }^{3}$ Other advantages of DALK include shorter postoperative steroid therapy, a reduced risk of intraocular infection due to a "closed eye" procedure, and earlier removal of sutures." 4

In a recent randomized clinical trial (RCT) of DALK vs PK we showed that the best spectacle-corrected visual acuity (BSCVA) was significantly better in the PK group at 3 and 6 months follow-up, but was not significantly different from the DALK group at 12 months follow-up. ${ }^{5}$ We hypothesized that the lower visual acuity may be due to the formation of interface haze at the donor-recipient stromal interface. This haze might result in an increase in intraocular straylight and a decrease in contrast sensitivity. ${ }^{6,7}$

The present study was performed to evaluate intraocular straylight, and contrast sensitivity after DALK and PK, and to correlate these quality of vision parameters to the visual outcomes.

\section{MATERIALS AND METHODS}

This randomized multicentre trial was conducted at 5 ophthalmic centers in the Netherlands. The institutional review boards of all participating centres have approved the study. The trial was performed in accordance to the principles of the Declaration of Helsinki, and informed consent was obtained from all patients.

Inclusion criteria were patients with keratoconus intolerant for contact lens wear and stromal opacifications not reaching Descemet's membrane and without concomitant endothelial disease. Exclusion criteria were best-corrected visual acuity greater than or equal to 20/50, keratoconus with previous hydrops or Descemet's rupture, or corneal stromal opacification reaching Descemet's membrane. The medical history was recorded and all patients underwent a comprehensive ophthalmic examination. Preoperative data included patient age, gender, refractive error, preoperative lens status and ocular comorbidities. 
All included eyes were randomly assigned to either the DALK or the PK group. The randomization code was generated using a permuted block size of 2 . The surgical techniques for DALK and PK have been described previously. ${ }^{5}$

Straylight was measured using a straylight meter (C-Quant, Oculus $\mathrm{GmbH}$, Wetzlar, Germany), which uses a compensation comparison method with a forced-choice technique. ${ }^{8}$ Clinical straylight measurement is a relatively new development to quantify quality of vision and was originally developed for visual acuities better than 0.7 logarithm of the minimum angle of resolution (logMAR). The straylight value was expressed as a logarithmic intraocular straylight $(\log (s))$ value. Higher values indicate more straylight and an increased sensitivity to glare. ${ }^{9}$ Two consecutive straylight measurements of the study eye and the non-study eye were performed, after which an average amount of $\log (\mathrm{s})$ was calculated. Straylight values were also compared to a control group obtained from a previous database, consisting of age-matched subjects with a clear cornea and no cataract. ${ }^{9}$ Eyes that were unable to perform the straylight test preoperatively due to turbidity and limited visual acuity were substituted by the highest preoperative log(s) plus 0.1 assigning a straylight value of $\log (\mathrm{s})=2.33$.

Contrast sensitivity was measured using the Pelli-Robson chart (Clement Clarke Ltd, Harlow, United Kingdom). ${ }^{10}{ }^{11}$ Patients were tested both monocularly and binocularly, using the best spectacle correction for distance vision, a testing distance of 1 meter and a chart luminance of 85 candelas $/ \mathrm{m}^{2}$. The last triplet of letters of which at least 2 letters were correctly seen was recorded and expressed as a logarithmic contrast sensitivity $(\log (c))$ value. Higher values indicate a better contrast sensitivity.

The uncorrected visual acuity (UCVA) and BSCVA were determined using the Early Treatment of Diabetic Retinopathy Study letter charts and were converted to logMAR values. Vision levels of counting fingers, hand movements, light perception, and no light perception were substituted by logMAR values of 1.7, 2.0, 2.5 and 3.0, respectively. All outcome measures were measured preoperatively and at 3,6 and 12 months follow-up.

\section{Sample size and statistical analysis}

The sample size calculation of the main outcome of this randomized clinical trial has been described previously. ${ }^{5}$ Data were described as mean \pm standard deviation for continuous variables and as individual counts and percentages for categorical variables. Differences between groups were analyzed using a Student's $t$ test for continuous data. The Pearson's chi-square test was used to compare categorical data. Comparisons of preoperative data and postoperative data within a group were performed using a linear regression model. Correlations were assessed using the Pearson correlation coefficient in case of normal distributed data, and using the Spearman test in case of abnormal distributed data. A $P$ value of less than 0.05 was considered to be statistically significant. 
Statistical analysis was performed using SPSS for Windows (version 16.0, SPSS, Inc., Chicago, IL).

\section{RESULTS}

Fifty-six eyes of 56 patients were included, with 28 patients in both the DALK and PK groups (figure 1). Five patients in the DALK group did not receive the allocated treatment and were converted to a PK due to Descemet's membrane perforations.Patient characteristics of the DALK and PK group are listed in table 1.

Preoperatively, 10 patients (35.7\%) in the DALK group and 8 patients (28.6\%) in the PK group required spectacle correction for distance vision. In both groups there were 5 patients (17.9\%) who required rigid contact lenses and 2 patients (7.1\%) who required sclera lenses for distance vision. The remaining patients used no correction for

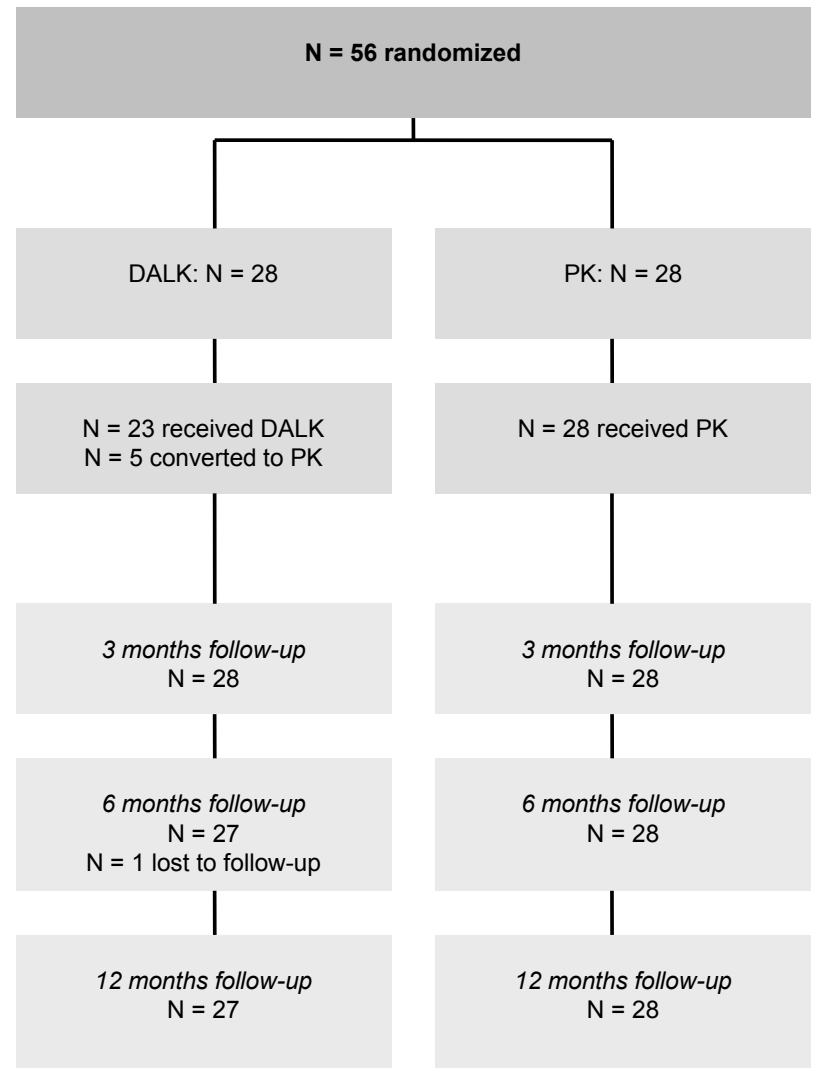

Figure 1. Participant Flow

DALK = deep anterior lamellar keratoplasty, $\mathrm{PK}=$ penetrating keratoplasty 


\begin{tabular}{llll}
\hline & DALK & PK & $P$ value \\
\hline Eyes $(\mathrm{N})$ & 28 & 28 & 0.899 \\
Age in years (mean $\pm \mathrm{SD})$ & $43.4 \pm 15.9$ & $42.9 \pm 14.1$ & $13(46 \%)$ \\
Women (N, \%) & $13(46 \%)$ & \\
Diagnosis & & $15(53.6 \%)$ \\
Keratoconus & & $9(32.1 \%)$ \\
Herpes simplex virus keratitis & $15(53.6 \%)$ & $1(3.6 \%)$ \\
Corneal ulcer & $7(25.0 \%)$ & $2(7.1 \%)$ \\
Pre-Descemet dystrophy & $6(21.4 \%)$ & $1(3.6 \%)$ \\
Groenouw stromal dystrophy & 0 & \\
Recipient lens status & 0 & $25(89.0 \%)$ \\
Phakic & & $3(11 \%)$ \\
Pseudophakic & $26(92.9 \%)$ & \\
\hline
\end{tabular}

DALK = deep anterior lamellar keratoplasty; $\mathrm{PK}=$ penetrating keratoplasty; $\mathrm{N}=$ number of eyes; $\mathrm{SD}=$ standard deviation

distance vision. Twelve months postoperatively, 8 patients $(28.6 \%)$ in the DALK group used spectacles, 1 patient (3.6\%) used rigid contact lenses, 2 patients $(7.1 \%)$ used sclera lenses, and 17 patients (60.7\%) did not use a correction for distance vision, with 7 of the 17 being unable to wear a correction because of anisometropia. In the PK group, 13 patients (46.4\%) used spectacles, 1 patient (3.6\%) used sclera lenses, and 14 patients $(50.0 \%)$ did not use a correction for distance vision with 7 of the 14 being unable to wear a correction because of anisometropia.

Preoperatively, $43 \%$ of patients had a visual acuity lower than the advised limit for straylight measurement (0.7 logMAR). Twelve of 28 patients (42.9\%) in both the DALK and PK groups were unable to complete the straylight test. The mean logMAR BSCVA in these DALK eyes was significantly higher in comparison with eyes of patients who did complete the test $(1.21 \pm 0.3 \log M A R$ vs $0.66 \pm 0.3 \log M A R, P<0.001)$. In the PK group the mean logMAR BSCVA of the eyes that were unable to complete the test was higher in comparison with eyes of patients who did complete the test, but this difference was not significant $(0.98 \pm 0.4 \log M A R$ vs $0.69 \pm 0.4 \log M A R, P=0.089)$.

In the present study population, preoperative straylight values were not significantly different between the DALK and PK groups (1.82 $\pm 0.5 \log (\mathrm{s})$ vs $1.80 \pm 0.5 \log (\mathrm{s})$, respectively, $P=0.917)$. During follow-up straylight values between the DALK and PK groups 

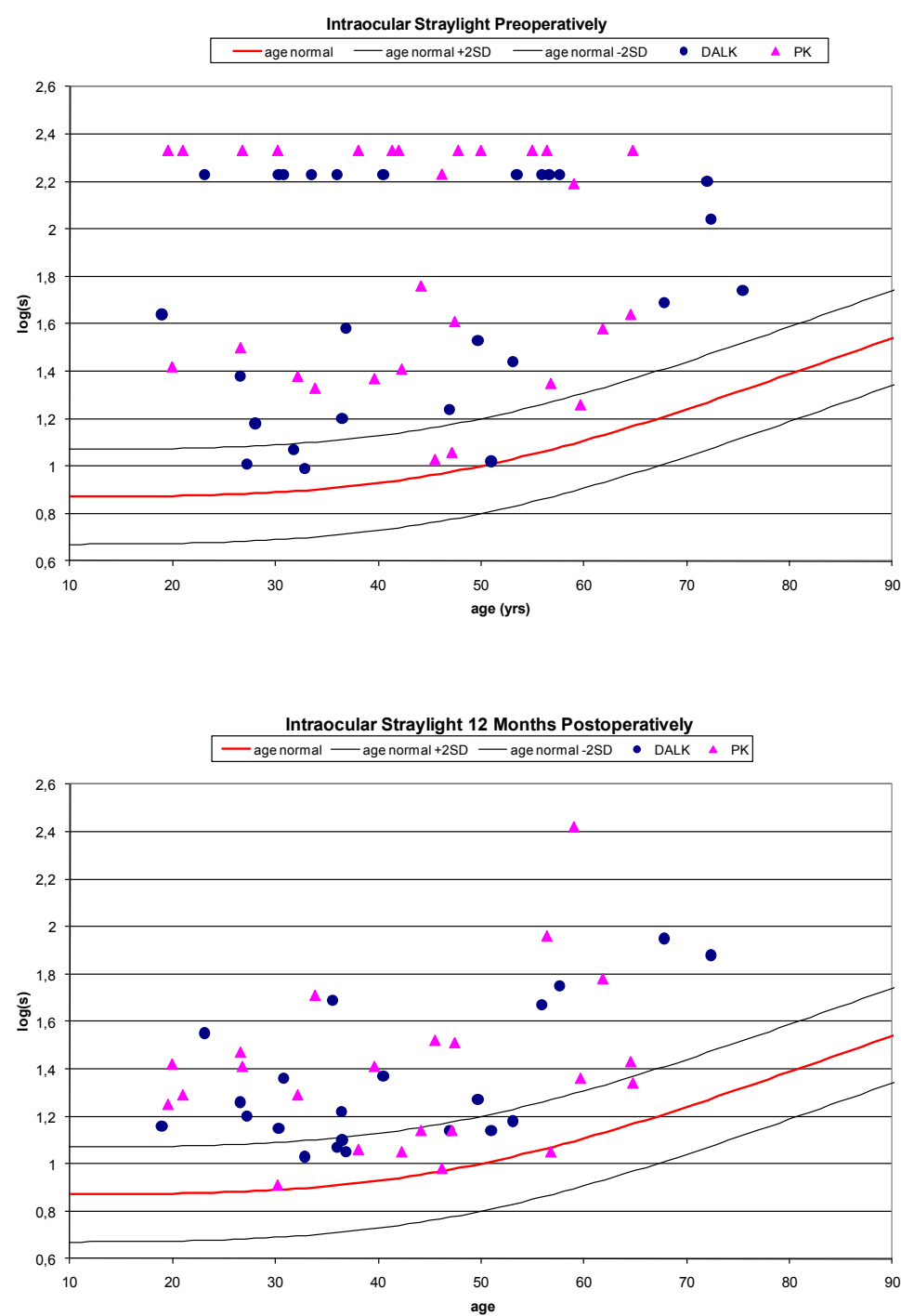

Figure 2. Intraocular Straylight Values as Function of Age for Deep Anterior Lamellar Keratoplasty versus Penetrating Keratoplasty

The lines represent mean levels of straylight \pm 2 SDs. DALK = deep anterior lamellar keratoplasty, PK = penetrating keratoplasty, $\mathrm{SD}=$ standard deviation, $\log (\mathrm{s})=$ logarithmic intraocular straylight value. 
were comparable at 3 months ( $1.32 \pm 0.2 \log (\mathrm{s})$ vs $1.27 \pm 0.2 \log (\mathrm{s}), P=0.443), 6$ months $(1.33 \pm 0.3 \log (\mathrm{s})$ vs $1.31 \pm 0.2 \log (\mathrm{s}), P=0.835)$, and 12 months $(1.34 \pm 0.3 \log (\mathrm{s})$ vs 1.38 $\pm 0.3 \log (\mathrm{s}), P=0.642)$. In both groups, there was a significant improvement in straylight from preoperatively to 12 months follow-up (DALK, $P<0.001$ and $P K, P<0.001$ ).

Straylight value as function of age is shown in figure 2. Preoperatively, 4 patients (14.3\%) in the DALK group and 3 patients (10.7\%) in the PK group had straylight values within the normal age-matched range, while remaining patients had higher straylight values. Twelve months postoperatively, $25.9 \%(n=7)$ of DALK eyes and $28.6 \%(n=8)$ of PK eyes had straylight values within the normal age-matched range, whereas other patients had higher straylight values. The change in intraocular straylight and BSCVA values from baseline to 12 months postoperatively showed a correlation in the DALK group $(r=0.694, P<0.001)$, but not in the PK group $(r=0.330, P=0.124)$.

Contrast sensitivity for both groups are shown in table 2. At baseline, 6 patients (21.4\%) in the DALK group and 5 patients (17.9\%) in the PK group were unable to see the highest contrast at 1 meter distance. In both the DALK and PK groups, contrast sensitivity of the study eye improved significantly after surgery. Preoperatively, there was a correlation between BSCVA and contrast sensitivity in the DALK group $(r=-0.725, P<0.01)$ and PK group ( $r=-0.477, P=0.021$ ). The change in contrast sensitivity from baseline to 12 months postoperatively showed a correlation with the change in BSCVA for both the DALK and PK groups $(r=-0.480, P=0.038$, and $r=-0.725, P<0.001$, respectively).

Refractive and visual outcomes are shown in table 3. In the DALK and PKP group, the refractive and topographic astigmatism were not correlated with straylight during the follow-up. 


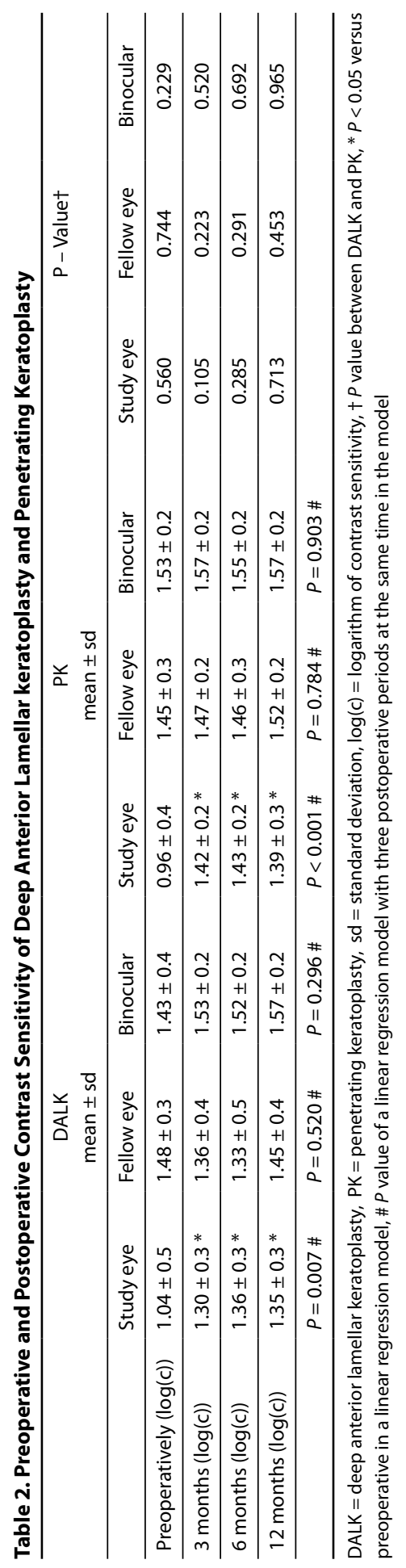




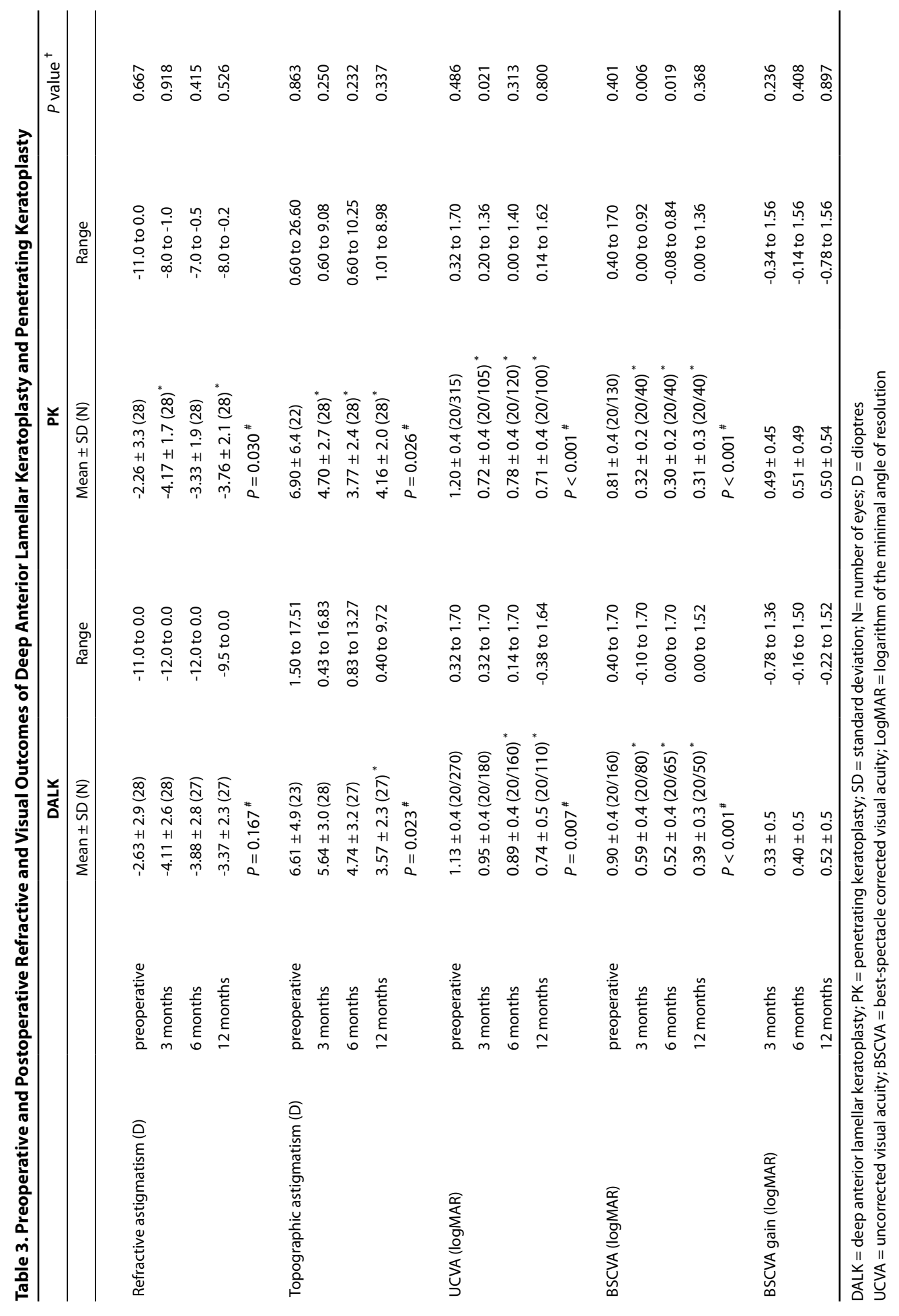




\section{DISCUSSION}

The purpose of this randomized multicenter trial was to evaluate the quality of vision (intraocular straylight and contrast sensitivity) after DALK in comparison with PK. Previous studies reported that the donor-recipient interface in lamellar keratoplasty may contribute to increased intraocular straylight, which may limit visual outcomes. ${ }^{7}$ Some studies reported no significant differences in visual acuity between DALK and PK, whereas others report better visual acuity after DALK. ${ }^{1,4}$, 5, 12-14 The main conclusion of our study is that both DALK and PK resulted in lower intraocular straylight values, better contrast sensitivity and comparable visual acuity after 12 months follow-up.

As has been explained on basic optical grounds, straylight and visual acuity derive from very different aspects of optical disturbances. ${ }^{15}$ Visual acuity is dominated by disturbances of aberrometric nature, whereas straylight is dominated by light scattering originating from volumetric scattering processes. Only, if an underlying disease process affects both aspects, a correlation will be found. ${ }^{9}$

Our results indicate that both DALK and PK are successful at improving intraocular straylight and result in comparable straylight values. The major improvement was seen from preoperatively to 3 months postoperatively, after which straylight values remained stable up to 12 months postoperatively. However, postoperative straylight values in both groups are still increased compared with age-matched controls.

From preoperatively to 12 months follow-up, the percentage of patients with straylight values within the normal age-matched range improved from $14.3 \%$ to $25.9 \%$ in the DALK group and from $10.7 \%$ to $28.6 \%$ in the PK group. Although there was a significant improvement in straylight after DALK and PK at 12 months follow-up, the majority of patients in both groups did not return to normal straylight levels. This finding was consistent with the results of patients after Descemet stripping endothelial keratoplasty and PK in comparison with normal eyes. ${ }^{16,}{ }^{17}$ Postoperative straylight values were compared with those of normal age-matched controls, but the age of the donor cornea was not age-matched with the patient. Our hypothesis was that the older transplant age probably could explain the increased postoperative straylight values of the study eyes. However, further analysis showed that an additional 3 eyes in the DALK group and 2 eyes in the PK group had straylight values within the band width of an age-matched population. At 12 months, there was a marked outlier (figure 2) in the PK group. The straylight value of this outlier may be explained by an increase in visually significant cataract during 12 months follow-up. After cataract surgery the straylight values of this patient decreased to $1.24 \log (\mathrm{s})$.

It is suggested that BSCVA after DALK may be limited as a result of increased straylight values due to the lamellar interface. ${ }^{18}$ In our study, the gain in BSCVA and the gain in straylight was comparable between the 2 groups. The correlation between the changes 
in straylight and BSCVA from baseline to 12 months postoperatively was statistically significant in the DALK group, but not in the PK group, which was comparable with the results of a previous study. ${ }^{6}$ Also, in a RCT of endothelial keratoplasty (manual technique vs mechanical microkeratome) and an RCT of Femtosecond laser assisted endothelial keratoplasty vs PK the interface did not result in different straylight levels between groups. $^{6,} 19$

Contrast sensitivity may be influenced by ocular pathologies, such as corneal edema, a corneal scar or cataract. In this study, we observed a significant improvement in contrast sensitivity following DALK and PK in a heterogeneous group of stromal disorders during the 12 months follow-up. The difference between the 2 groups was not significant. Other randomized and nonrandomized studies on DALK and PK also showed similar improvements in contrast sensitivity., 18, 20, 21 Furthermore, the contrast sensitivity in our study improved during follow-up, but remained lower in comparison with normal agematched controls. ${ }^{22}$ Other studies evaluating PK in patients with keratoconus reported similar results. ${ }^{23}$ Improvements in BSCVA correlated with improvements in contrast sensitivity after DALK and PK. The lamellar interface in the DALK group did not result in significant differences in contrast sensitivity in comparison with the PK group. The largest improvement in contrast sensitivity was seen in the first 3 months postoperatively, which was also in accordance with improvements in other visual outcomes (UCVA, BSCVA) and improvements in straylight.

The refractive and topographic astigmatism were comparable between the DALK and PK groups, and there was no significant correlation between straylight and astigmatism in both groups. These results were consistent with those of Rozema et al. who did not find a correlation between straylight and astigmatism in healthy eyes. ${ }^{24}$

Initially, the visual outcomes in our study were significantly better in the PK group compared with the DALK group. However, at 12 months postoperatively the BSCVA in the DALK and PK groups was comparable. This indicates that Anwar's big-bubble technique, which achieves a deep dissection down to the level of the Descemet's membrane, does not lead to extensive interface haze. Earlier stabilization of visual outcomes after PK, compared with DALK, has been suggested by a previous study. ${ }^{13}$ Even though a significantly better BSCVA after DALK has been reported, the majority of studies reported similar results in DALK eyes compared with PK eyes." ${ }^{1,14}$ Some studies have reported better visual outcomes following PK compared with DALK, but this might be due to incomplete stromal removal in DALK eyes. ${ }^{20,25}$

In conclusion, this randomized clinical trial showed that DALK resulted in similar improvements in straylight and contrast sensitivity compared with PK. Our results show that straylight, contrast sensitivity, and visual acuity, after DALK is comparable with that attained after PK and that the interface in DALK does not result in a decreased quality of vision. 


\section{REFERENCES}

1. Bahar I, Kaiserman I, Srinivasan S, et al. Comparison of three different techniques of corneal transplantation for keratoconus. Am J Ophthalmol 2008;146:905-12 e901.

2. Patel SV, Hodge DO, Bourne WM. Corneal endothelium and postoperative outcomes 15 years after penetrating keratoplasty. Am J Ophthalmol 2005;139:311-19.

3. Borderie VM, Sandali O, Bullet J, et al. L. Long-term results of deep anterior lamellar versus penetrating keratoplasty. Ophthalmology 2012;119:249-55.

4. Shimazaki J, Shimmura S, Ishioka M, et al. Randomized clinical trial of deep lamellar keratoplasty vs penetrating keratoplasty. Am J Ophthalmol 2002;134:159-65.

5. Cheng YY, Visser N, Schouten JS, et al. Endothelial cell loss and visual outcome of deep anterior lamellar keratoplasty versus penetrating keratoplasty: a randomized multicenter clinical trial. Ophthalmology 2011;118:302-9.

6. Patel SV, McLaren JW, Hodge DO, et al. Scattered light and visual function in a randomized trial of deep lamellar endothelial keratoplasty and penetrating keratoplasty. Am J Ophthalmol 2008;145:97-105.

7. Rich LF. Expanding the scope of lamellar keratoplasty. Trans Am Ophthalmol Soc 1999;97:771-14.

8. Franssen L, Coppens JE, van den Berg TJ. Compensation comparison method for assessment of retinal straylight. Invest Ophthalmol Vis Sci 2006;47:768-76.

9. Van Den Berg TJ, Van Rijn LJ, Michael R, et al. Straylight effects with aging and lens extraction. Am J Ophthalmol 2007;144:358-63.

10. Elliott DB, Sanderson K, Conkey A. The reliability of the Pelli-Robson contrast sensitivity chart. Ophthalmic Physiol Opt 1990;10:21-4.

11. Pelli DG RJ, Wilkens AJ. The design of a new letter chart of measuring contrast sensitivity. Clinical Vision Science 1988;2:187-99.

12. Reinhart WJ, Musch DC, Jacobs DS, et al. Deep anterior lamellar keratoplasty as an alternative to penetrating keratoplasty a report by the american academy of ophthalmology. Ophthalmology 2011;118:209-18.

13. Kawashima M, Kawakita T, Den S, et al. Comparison of deep lamellar keratoplasty and penetrating keratoplasty for lattice and macular corneal dystrophies. Am J Ophthalmol 2006;142:304-9.

14. Tan DT, Anshu A, Parthasarathy A, et al. Visual acuity outcomes after deep anterior lamellar keratoplasty: a case-control study. Br J Ophthalmol 2010;94:1295-99.

15. van den Berg TJ FLaCJ. Clarity and Straylight. Oxford, 2010:173-83.

16. Patel SV, Baratz KH, Hodge DO, et al. The effect of corneal light scatter on vision after Descemet stripping with endothelial keratoplasty. Arch Ophthalmol 2009;127:153-60.

17. Patel SV, McLaren JW, Hodge DO, et al. The effect of corneal light scatter on vision after penetrating keratoplasty. Am J Ophthalmol 2008;146:913-19.

18. Fontana L, Parente $G$, Sincich A, et al. Influence of graft-host interface on the quality of vision after deep anterior lamellar keratoplasty in patients with keratoconus. Cornea 2011;30:497-2.

19. Cheng YY, van den Berg TJ, Schouten JS, et al. Quality of vision after femtosecond laser-assisted descemet stripping endothelial keratoplasty and penetrating keratoplasty: a randomized, multicenter clinical trial. Am J Ophthalmol 2011;152:556-66 e551.

20. Ardjomand N, Hau S, McAlister JC, et al. Quality of vision and graft thickness in deep anterior lamellar and penetrating corneal allografts. Am J Ophthalmol 2007;143:228-35. 
21. Akdemir MO, Kandemir B, Sayman IB, et al. Comparison of contrast sensitivity and visual acuity between deep anterior lamellar keratoplasty and penetrating keratoplasty in patients with keratoconus. International journal of ophthalmology 2012;5:737-41.

22. Mantyjarvi M, Laitinen T. Normal values for the Pelli-Robson contrast sensitivity test. J Cataract Refract Surg 2001;27:261-66.

23. Pesudovs $\mathrm{K}$, Schoneveld $\mathrm{P}$, Seto RJ, et al. Contrast and glare testing in keratoconus and after penetrating keratoplasty. Br J Ophthalmol 2004;88:653-57.

24. Rozema JJ, Van den Berg TJ, Tassignon MJ. Retinal straylight as a function of age and ocular biometry in healthy eyes. Invest Ophthalmol Vis Sci 2010;51:2795-99.

25. Funnell CL, Ball J, Noble BA. Comparative cohort study of the outcomes of deep lamellar keratoplasty and penetrating keratoplasty for keratoconus. Eye (Lond) 2006;20:527-32. 



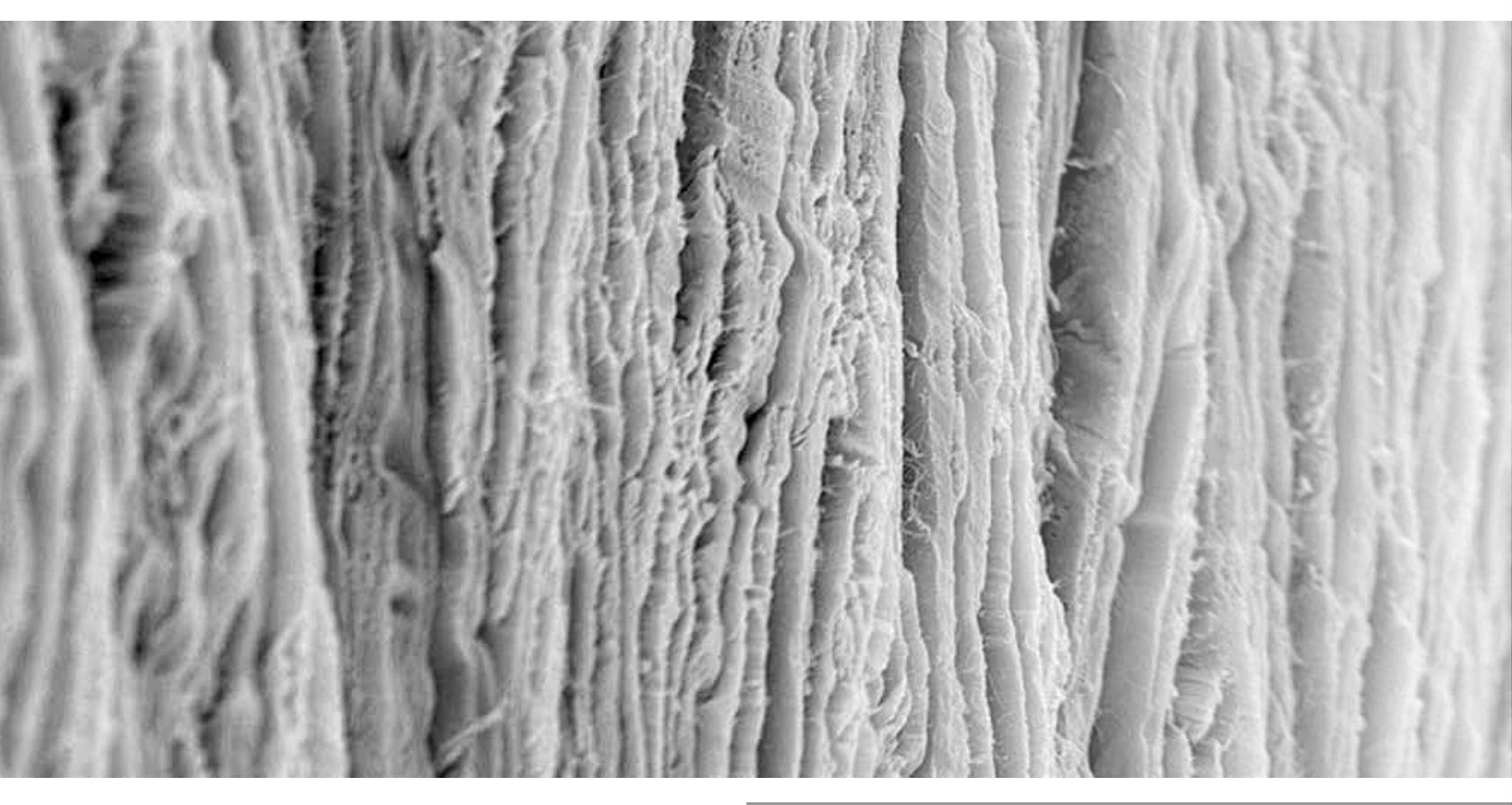




\title{
CHAPTER 12
}

Economic Evaluation of Deep

Anterior Lamellar Keratoplasty

Versus Penetrating Keratoplasty in

The Netherlands

\author{
Frank van den Biggelaar \\ Yanny Y.Y. Cheng \\ Rudy M.M.A. Nuijts \\ Jan S. Schouten \\ Robert-Jan Wijdh \\ Elisabeth Pels \\ Hugo van Cleynenbreugel \\ Catharina A. Eggink \\ Michel J.W. Zaal \\ Wilhelmina J. Rijneveld \\ Carmen D. Dirksen
}

Am J Ophthalmol. 2011 Mar;151(3):449-59 


\section{ABSTRACT}

Purpose: To evaluate the cost effectiveness of deep anterior lamellar keratoplasty (DALK) versus penetrating keratoplasty (PK) in The Netherlands.

Design: Cost-effectiveness analysis alongside a randomized, multicenter clinical trial.

Methods: Fifty-three patients with corneal stromal pathologic features not affecting the endothelium were included with 28 patients in the DALK group and 25 in the PK group. Quality of life was measured before surgery and 3,6, and 12 months after surgery. The main outcome measures were incremental cost-effectiveness ratios per clinically improved patient on the 25-item National Eye Institute Visual Functioning Questionnaire and per patient with endothelial cell loss of maximally $20 \%$ within the first year.

Results: Mean total bootstrapped costs per patient were $€ 7607$ (US\$10 498) in the DALK group and $€ 6552$ (US\$9042) in the PK group. The incremental cost-effectiveness ratios were $€ 9977$ (US\$13 768) per clinically improved patient on the 25-item National Eye Institute Visual Functioning Questionnaire and $€ 6900$ (US\$9522) per patient with cell loss of maximally $20 \%$. In patients without perforation of the Descemet membrane, the incremental cost-effectiveness ratio was $€ 5250$ (US\$7245) per patient.

Conclusions: This study shows that DALK is more costly and more effective as compared with PK. Results on the 25-item National Eye Institute Visual Functioning Questionnaire were in favor of DALK, and endothelial cell loss in DALK patients remained stable after 6 months, whereas cell loss in PK patients continued. Furthermore, DALK procedures performed without perforation of the Descemet membrane were more effective. However, because it is unknown what society is willing to pay for an additional improved patient, cost effectiveness of DALK within a limited follow-up period of 12 months is unclear. Cost effectiveness of DALK may improve over time because of lower graft failure. 


\section{INTRODUCTION}

For many years, penetrating keratoplasty (PK) has been the standard treatment for diseases of corneal stroma with unaffected endothelium such as keratoconus, infectious keratitis, and stromal dystrophies. ${ }^{1-4}$ Although it has been proven to be a safe and effective technique that achieves good visual outcomes, ${ }^{5}$ graft failure is reported in $18 \%$ to $34 \%$ of the patients. ${ }^{5-7}$ Graft failure is caused mainly by endothelial rejection and endothelial failure, which account for more than $50 \%$ of graft failures. ${ }^{7}$ After PK, endothelial cell counts have been shown to drop by approximately $70 \%$ at 5 to 10 years, $^{7,8}$ having a major impact on long-term graft survival.

In the last decade, interest has grown in lamellar transplantation techniques in which only the anterior side of the cornea is transplanted. The main clinical aim to develop lamellar techniques was to preserve the endothelial layer, assuming that this will reduce the risk of endothelial rejection and will prevent endothelial cell loss of the corneal graft. Postoperative visual function should be comparable with PK to justify lamellar techniques. ${ }^{9,10}$ In deep anterior lamellar keratoplasty (DALK), the corneal stroma tissue is removed down to the Descemet membrane or a thin layer of stroma is left on top of the Descemet membrane. The corneal stroma is replaced by donor tissue. The healthy Descemet membrane and endothelium of the recipient is not grafted, which reduces the risk of graft failure. Furthermore, long-term endothelial cell loss is lower after DALK as compared with $\mathrm{PK}^{4,9,11}$ However, the procedure is technically challenging and may result in perforation of the Descemet membrane in a significant number of cases (range, $4 \%$ to $39 \%))^{3,4,12-14}$

Although several studies evaluated the efficacy and visual outcomes of DALK versus $\mathrm{PK}^{3,4,9,15}$ the efficiency of the techniques has not yet been established. An economic evaluation is a method to compare outcomes and costs of interventions ${ }^{16}$ with the aim to improve resource allocation decisions by policy makers and insurers. One type of economic evaluation is the cost-effectiveness analysis, in which the costs of an intervention are related to a single outcome measure, for example clinical success. ${ }^{17}$ In this study, a cost-effectiveness analysis was performed, comparing DALK with PK.

\section{METHODS}

\section{Study population}

The trial-based economic evaluation was conducted alongside the Dutch Lamellar Corneal Transplantation Study, a randomized multicenter clinical trial that evaluated the efficacy and safety of DALK versus PK. Patients were included between July 2005 and 
February 2008 at 5 clinical centers in The Netherlands. Informed consent was obtained from all patients.

Inclusion criteria were patients with keratoconus intolerant of contact lens wear (without prior hydrops or Descemet rupture) and stromal opacifications not reaching the Descemet membrane and without concomitant endothelial disease. Exclusion criteria were best-corrected visual acuity more of $20 / 50$ or worse, keratoconus with previous hydrops or Descemet rupture, or corneal stromal opacification reaching the Descemet membrane.

In each medical center, consecutive cases were assigned randomly to treatment by DALK or by PK. A permuted block size of 2 was used to generate the randomization code. All patients underwent a comprehensive ophthalmic examination and completed a vision-related quality-of-life questionnaire. Data were obtained at randomization and at 3,6 , and 12 months after the transplantation, which is $4.5,7.5$, and 13.5 months after baseline measurement, respectively.

In the Dutch Lamellar Corneal Transplantation Study, the sample size was calculated based on an endothelial cell loss of $8 \%$ after DALK and $45 \%$ after PK in 1 year, based on our preliminary experiences and reported amounts of endothelial cell loss in previous studies. ${ }^{11,18,19}$ Assuming an a of 0.05 , a power of $90 \%$, and $10 \%$ loss to follow-up, 28 patients were needed in each treatment group.

\section{Surgical procedures}

In the DALK group, the surgical procedure was performed using the big-bubble technique described previously. ${ }^{20,21}$ Partial thickness trephination of approximately $60 \%$ depth was performed,followed by a partial-thickness anterior keratectomy. An air bubble was inserted into the remaining stroma using a 30-gauge needle. An incision was made in the anterior wall of the bubble and a blunt spatula was used to form 4 sections in the anterior stromal layers that then were excised with blunt-tipped microscissors. A donor button subsequently was sutured into the bed using 8 interrupted 10-0 nylon sutures and a continuous 11-0 nylon suture.

In the PK group, the recipient cornea was trephined using a 7.75- or 8.0-mm HessburgBarron vacuum trephine (Jed Med, St Louis, Missouri), and the donor cornea was trephined with an 8.0- or 8.25-mm Hessburg-Barron trephine. In all patients, a combined suturing technique of 8 interrupted 10-0 nylon sutures with a continuous $11-0$ nylon suture was used. Selective suture removal was based on the topographic astigmatism pattern.

\section{Economic evaluation}

The economic evaluation was performed from a health care perspective with a time horizon of 13.5 months, from randomization (approximately 1.5 months before surgery) 
up to 12 months after surgery. All relevant resources consumed within the health care sector were taken into account. Effectiveness was expressed in 2 ways. First, the 25 -item National Eye Institute Visual Functioning Questionnaire (NEI VFQ-25) was used, which is specified for vision related quality-of-life. ${ }^{22}$ Second, because preservation of the host endothelium is the main advantage of DALK, the amount of endothelial cell loss was used as an outcome measure. Incremental cost-effectiveness ratios (ICERs) were calculated by dividing the difference in costs (DALK minus PK) by the difference in effectiveness (DALK minus PK) between the 2 treatments.

Twenty-five-item National Eye Institute Visual Functioning Questionnaire. The NEI VFQ25 measures generic health dimensions and task-oriented domains related to daily visual functioning that are most important for patients with a chronic eye disease. The NEI VFQ-25 consists of 25 questions that can be expanded with 13 additional items. The questions comprise 12 subscales, of which 11 eventually can be converted into a single score. The remaining subscale (general health) is included in the questionnaire to provide robust information about an individual's general health status. After recoding, all scores range from 0 (worst possible value) to 100 (best possible value).

Several studies have suggested that a 10-point change in the composite score is clinically important. ${ }^{23-25}$ Therefore, a patient with a minimum gain of 10 points in the composite score at 12 months after surgery was considered to be clinically improved. An ICER was calculated that expressed the incremental costs per clinically improved patient.

Endothelial cell loss. Endothelial cell densities were measured at randomization and at 3, 6, and 12 months after surgery using the specular microscopy Noncon Robo SP 8000 (Konan, Hyogo, Japan). To reduce the sampling error, 3 specular photographs were obtained at each measurement and each image was analyzed by selecting 50 cells in the center of the image. Studies show an endothelial cell loss in the first year ranging from $6 \%$ to $20 \%$ after DALK surgery ${ }^{4,26-28}$ and from $20 \%$ to $43 \%$ after PK surgery. ${ }^{4,9,29,30}$ In this study, an ICER was calculated that expressed the incremental costs per patient with an endothelial cell loss of maximally $20 \%$ within the first year after surgery.

\section{Cost analysis}

All direct medical costs of the 2 interventions were assessed according to the Dutch guidelines for cost calculations. ${ }^{31}$ Because direct nonmedical costs and indirect costs were assumed to be equal in both intervention groups, these costs were excluded from analyses. Costs were calculated by multiplying the volumes of resource use by the cost price per resource unit. All costs were converted to 2008 Euros, and costs occurring after 12 months were discounted at an annual rate of $4 \%$. All costs are reported in Euros $(€)$ and United States dollars (US\$) ( $€ 1=$ US\$1.38). ${ }^{32}$

Resources included preparation, preservation, allocation, and transportation of the donor corneas, which were provided by the Bio Implant Services Foundation; outpatient 
visits; diagnostic procedures; surgical procedures; hospitalization; and postoperative drugs use. Data for outpatient visits, surgical and diagnostic procedures, and hospitalization were obtained from the registries of the participating hospitals.

The services of the Bio Implant Services Foundation were valued using Dutch reimbursement rates, because no unit prices were available. Costs of outpatient visits, surgical procedures, hospitalization, and diagnostic procedures were valued using standardized integral unit prices (consisting of personnel, material, capacity, and overhead costs) as calculated by Maastricht University Medical Centre in which $68 \%$ of the patients were included. Integral costs prices of surgical procedures depended on the operation time per patient and were allocated to 2 cost drivers, namely general operating room costs and specific ophthalmology costs. Costs of postoperative drugs were obtained from the Dutch Pharmacotherapeutic Compass. ${ }^{33}$

\section{Statistical analysis}

Outcomes were analyzed according to the intention-to-treat principle. Incomplete data of the NEI VFQ-25 (5\%), incomplete cost data (3\%), and incomplete data considering the endothelial cell density (22\%) and visual acuity (0.4\%) were imputed using SPSS Multiple Imputation software version 17.0 for Windows (SPSS, Inc, Chicago, Illinois, USA), under the assumption that the data were missing at random. Patients who had not completed the baseline NEI VFQ-25 were not included in the economic evaluation. A linear regression model was used with a total run length of 100 iterations. Covariates included in the imputation model were manifest refraction, refractive astigmatism, and topographic astigmatism, which were obtained in the Dutch Lamellar Corneal Transplantation Study at randomization and at 3, 6, and 12 months after surgery. Furthermore, age, sex, randomization group, and results on additional questions to measure patient satisfaction after a corneal transplantation were included in the model. Five imputed data sets were obtained. All analyses were performed with each of these data sets and the results were pooled.

Because cost data generally are highly skewed, traditional parametric and nonparametric statistical methods are not appropriate to analyze the difference in mean costs between groups. ${ }^{34}$ Therefore, to estimate the uncertainty in costs and effects, nonparametric bootstrapping with 1000 replications was performed on the incremental costs and effects, using Microsoft Excel for Windows (Microsoft Corp, Redmond, Washington, USA). The bootstrapped incremental cost-effectiveness ratios were plotted in a cost-effectiveness plane in which the horizontal axis represents the difference in effects between the 2 treatment groups and the vertical axis represents the difference in costs between the 2 treatment groups (Figure 1). Cost-effectiveness pairs can be located in 1 of 4 areas: (1) in the southeast area, DALK is dominant over PK (less costly and more effective); (2) in the northwest area, DALK is inferior over PK (more costly and less effec- 


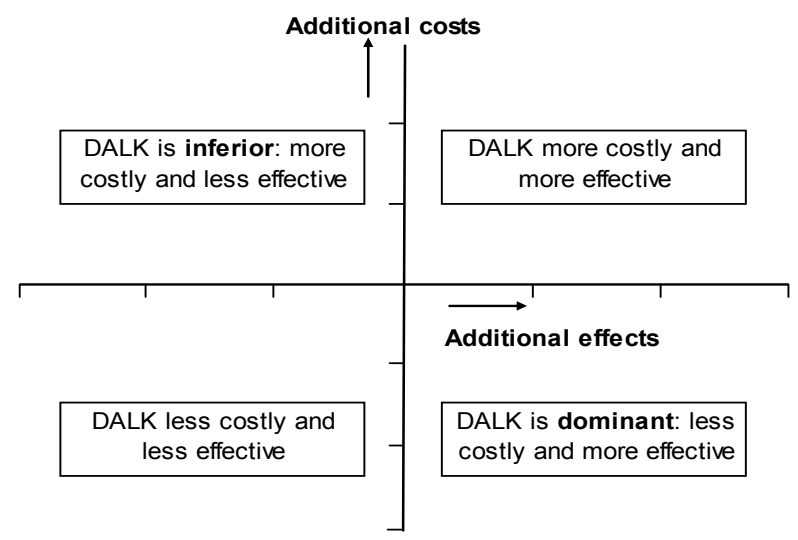

FIGURE 1. Cost-effectiveness plane for deep anterior lamellar keratoplasty (DALK) versus penetrating keratoplasty (PK).

tive); (3) in the southwest area, DALK is less costly and less effective as compared with PK; (4) in the northeast area, DALK is more costly and more effective as compared with PK. In addition, cost-effectiveness acceptability curves were created, showing the probability that DALK is cost-effective using a range of ceiling ratios, which represents the maximum amount of money that the decision maker is willing to pay for an additional health effect (ie, a clinically improved patient).

\section{Secondary analyses}

To test the robustness of the results, secondary analyses were performed. First, an additional analysis was performed in which we excluded the patients with perforation of the Descemet membrane. Second, we replaced the cost prices as determined in the Maastricht University Medical Centre by standardized Dutch unit prices-if available- determined by Oostenbrink and associates. ${ }^{31}$ Third, several studies stated that a 5-point change in the composite score of the NEI VFQ-25 is clinically relevant. ${ }^{23,24}$ Therefore, we considered a patient with a gain of 5 points in the composite score at 12 months after surgery to be clinically improved. Finally, 5 patients who were randomized to DALK required conversion to PK. Therefore, instead of an intention-to-treat analysis, an as-treated analysis was conducted in our secondary analysis, in which the converted patients were analyzed in the PK group.

\section{RESULTS}

A total of 56 patients were eligible for inclusion in the study. In 3 patients (5.4\%), baseline data on the NEI VFQ-25 were missing. Consequently, a total of 53 eyes in 53 patients were included in the economic evaluation, with 28 patients in the DALK group and 25 
in the PK group. Because of perforations of the Descemet membrane, 5 patients in the DALK group did not receive the allocated treatment and were converted to PK. However, according to the intention-to-treat principle, these patients were analyzed in the DALK group. In the PK group, all patients received the allocated treatment.

In Table 1, the baseline characteristics of the patients included in the study are shown. The mean age was $43.4 \pm 15.9$ years and $42.9 \pm 14.1$ years in the DALK group and the PK group, respectively $(P=.9)$. Twenty-seven patients in the DALK group and 22 patients in the PK group were phakic, which is not significantly different $(P=.26)$. In both treatment groups, the main reason for keratoplasty was keratoconus and corneal scarring caused by herpes simplex virus keratitis. The mean baseline composite score on the NEI VFQ-25 was 63.5 and 68.3 in the DALK and PK groups, respectively $(P=.33)$.

In Table 2, visual outcomes are displayed, showing comparable preoperative UCVA and BSCVA in the DALK and PK group. At 12 months after surgery, UCVA, BSCVA, and BSCVA gain were not significantly different between both groups. Further details of this population have been described elsewhere. ${ }^{35}$

\section{Effectiveness}

In Figure 2, mean outcomes on the NEI VFQ-25 composite scale and mean endothelial cell losses are displayed for the 2 treatment groups at the four measurements. At 12 months after surgery, pooled data showed that $59 \%$ of the patients were clinically improved (minimum gain of 10 points in the NEI VFQ-25 composite score) in the DALK group and $48 \%$ in the PK group. An endothelial cell loss of maximally $20 \%$ within the first

Table 1 . Baseline Characteristics of Study Population $(n=53)$ DALK versus PK

\begin{tabular}{llll}
\hline & DALK & PK & P value \\
\hline Eyes $(\mathbf{n})$ & 28 & 25 & 0.9 \\
Age in yrs (mean \pm SD) & $43.4 \pm 15.9$ & $42.9 \pm 14.1$ & 0.86 \\
Women (n, \%) & $13(46 \%)$ & $11(44 \%)$ & 0.26 \\
Recipient lens status & & & \\
Phakic & $27(96 \%)$ & $22(88 \%)$ & $3(12 \%)$ \\
Pseudophakic & $1(4 \%)$ & & \\
Diagnosis & & $14(56 \%)$ & \\
Keratoconus & $15(54 \%)$ & $1(4 \%)$ & \\
Herpes simplex virus keratitis & $7(25 \%)$ & $2(8 \%)$ & \\
Corneal ulcer & $6(21 \%)$ & 6.33 \\
Pre-Descemet dystrophy & 0 & $68.3 \pm 15.2$ \\
Groenouw stromal dystrophy & 0 & $63.5 \pm 20.1$ & \\
Composite score NEI VFQ-25 (mean \pm SD) & & & \\
\hline
\end{tabular}

DALK = deep anterior lamellar keratoplasty; NEI VFQ-25 = 25-item National Eye Institute Visual Functioning Questionnaire; $\mathrm{PK}=$ penetrating keratoplasty; $\mathrm{SD}=$ standard deviation. 
Table 2. Preoperative and Postoperative Visual Outcomes for DALK versus PK (Pooled Data)

\begin{tabular}{|c|c|c|c|}
\hline & DALK & PK & $P$ value $^{\mathrm{a}}$ \\
\hline & Mean \pm SD & Mean \pm SD & \\
\hline \multicolumn{4}{|c|}{ UCVA (logMAR) } \\
\hline Preoperative & $1.13 \pm 0.07(20 / 270)$ & $1.20 \pm 0.07(20 / 315)$ & 0.49 \\
\hline 3 months & $0.95 \pm 0.07(20 / 180)$ & $0.72 \pm 0.07(20 / 105)^{b}$ & 0.02 \\
\hline 6 months & $0.89 \pm 0.08(20 / 160)^{b}$ & $0.78 \pm 0.08(20 / 120)^{b}$ & 0.32 \\
\hline 12 months & $0.74 \pm 0.08(20 / 110)^{b}$ & $0.71 \pm 0.08(20 / 100)^{b}$ & 0.79 \\
\hline \multicolumn{4}{|c|}{ BSCVA (logMAR) } \\
\hline Preoperative & $0.90 \pm 0.08(20 / 160)$ & $0.81 \pm 0.08(20 / 130)$ & 0.4 \\
\hline 3 months & $0.59 \pm 0.08(20 / 80)^{b}$ & $0.32 \pm 0.04(20 / 40)^{b}$ & 0.01 \\
\hline 6 months & $0.52 \pm 0.07(20 / 65)^{b}$ & $0.30 \pm 0.05(20 / 40)^{b}$ & 0.02 \\
\hline 12 months & $0.39 \pm 0.06(20 / 50)^{b}$ & $0.31 \pm 0.07(20 / 40)^{b}$ & 0.37 \\
\hline \multicolumn{4}{|c|}{ BSCVA gain (logMAR) } \\
\hline 3 months & $0.32 \pm 0.1$ & $0.49 \pm 0.09$ & 0.19 \\
\hline 6 months & $0.39 \pm 0.09$ & $0.51 \pm 0.1$ & 0.34 \\
\hline 12 months & $0.52 \pm 0.08$ & $0.50 \pm 0.11$ & 0.92 \\
\hline
\end{tabular}

BSCVA = best spectacle-corrected visual acuity; DALK = deep anterior lamellar keratoplasty; logMAR = logarithm of the minimal angle of resolution; $\mathrm{PK}=$ penetrating keratoplasty; $\mathrm{UCVA}=$ uncorrected visual acuity.

${ }^{\mathrm{a}} P$ value between DALK and PK (t test).

${ }^{\mathrm{b}} P<.05$ between one of the postoperative visits and the preoperative visit (linear regression model).

year was found in $39 \%$ of the patients in the DALK group and $24 \%$ of the patients in the PK group. In Table 3, the outcomes on the NEI VFQ-25 questionnaire are shown. In both groups, scores on all subscales increased at 12 months after surgery as compared with randomization. 


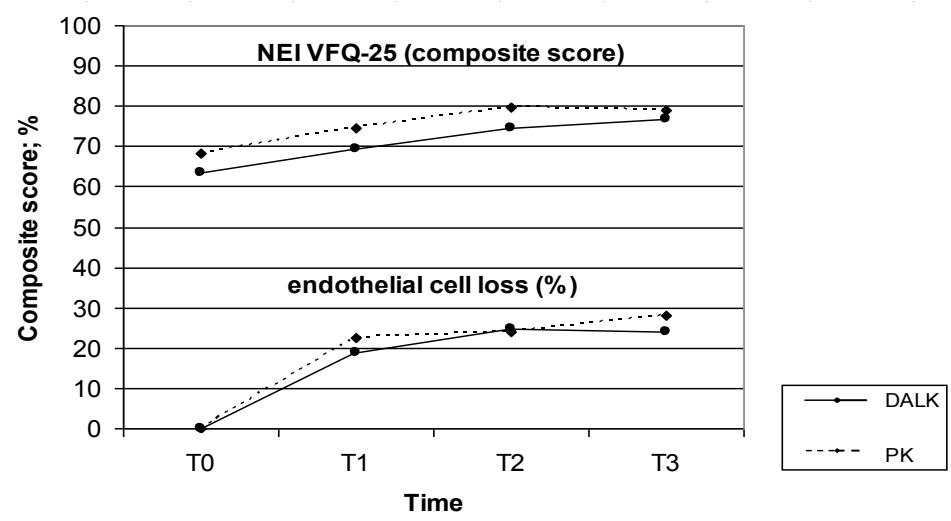

FIGURE 2. Graph showing the effectiveness outcomes for deep anterior lamellar keratoplasty (DALK) versus penetrating keratoplasty (PK). NEI VFQ-25 = 25-item National Eye Institute Visual Functioning Questionnaire; T0 = preoperative measurement; $\mathrm{T} 1$ = 3-month postoperative measurement; $\mathrm{T} 2$ = 6-month postoperative measurement; $\mathrm{T} 3$ = 12-month postoperative measurement.

Table 3. Outcomes on NEI VFQ-25 for DALK versus PK

\begin{tabular}{lcccccccccccc}
\hline & \multicolumn{2}{c}{ T0 } & \multicolumn{2}{c}{ T1 } & \multicolumn{2}{c}{ T2 } & & T3 & \multicolumn{3}{c}{$\begin{array}{c}\text { Change scores } \\
\text { T3 - T0 }\end{array}$} \\
\hline & DALK & PK & DALK & PK & DALK & PK & DALK & PK & DALK & PK \\
\hline NEI VFQ-25 & & & & & & & & & & \\
subscales & & & & & & & & & & \\
General vision & 50.4 & 51.4 & 53.2 & 56.2 & 59.7 & 61.4 & 58.1 & 60.2 & 7.8 & 8.8 \\
Ocular pain & 51.8 & 59.5 & 59.2 & 70.0 & 70.3 & 75.5 & 69.9 & 76.5 & 18.1 & 17.0 \\
Near activities & 64.3 & 67.8 & 70.8 & 79.7 & 76.2 & 81.8 & 79.2 & 82.0 & 14.9 & 14.2 \\
Distance activities & 61.9 & 67.0 & 69.1 & 73.5 & 75.7 & 79.7 & 77.3 & 80.5 & 15.4 & 13.5 \\
Social functioning & 81.8 & 89.3 & 85.1 & 91.0 & 88.2 & 92.3 & 90.7 & 92.7 & 8.8 & 3.3 \\
Mental health & 60.2 & 63.4 & 68.7 & 72.2 & 73.3 & 77.8 & 74.6 & 72.4 & 14.4 & 9.0 \\
Role difficulties & 55.1 & 56.8 & 60.8 & 66.8 & 67.7 & 73.3 & 71.3 & 68.3 & 16.2 & 11.5 \\
Dependency & 77.9 & 83.5 & 84.1 & 87.5 & 85.7 & 89.8 & 89.4 & 89.0 & 11.5 & 5.5 \\
Driving & 50.1 & 56.9 & 53.7 & 54.8 & 55.6 & 71.7 & 65.5 & 70.9 & 15.4 & 14.0 \\
Color vision & 88.9 & 89.0 & 90.0 & 92.0 & 93.4 & 95.0 & 91.6 & 96.0 & 2.7 & 7.0 \\
Peripheral vision & 58.9 & 67.0 & 66.3 & 76.0 & 74.6 & 79.0 & 77.0 & 79.0 & 18.0 & 12.0 \\
Composite score & 63.6 & 68.3 & 69.2 & 74.5 & 74.6 & 79.8 & 76.8 & 78.9 & 13.2 & 10.5 \\
General health & 62.9 & 61.4 & 65.3 & 63.4 & 64.8 & 65.8 & 64.1 & 60.5 & 1.2 & -0.9 \\
\hline DAlK=d
\end{tabular}

DALK = deep anterior lamellar keratoplasty; NEI VFQ-25 = 25-item National Eye Institute Visual Functioning Questionnaire; $\mathrm{PK}=$ penetrating keratoplasty; $\mathrm{T} 0=$ preoperative measurement $; \mathrm{T} 1=3-$ month postoperative measurement; $\mathrm{T} 2=6$-month postoperative measurement; $\mathrm{T} 3=12$-month postoperative measurement. 


\section{Costs}

Table 4 shows the mean resource use andpooled costs per patient. Mean total costs per patient were $€ 7607$ (US\$10 498) in the DALK group compared with $€ 6552$ (US\$9042) in the PK group. Differences in costs were caused mainly by the costs in the surgical procedures. Preoperative costs accounted for the highest costs in both groups, but were comparable.

Table 4: Mean resource use and pooled costs per patient $(n=53)$ for DALK versus PK

\begin{tabular}{|c|c|c|c|c|c|}
\hline & \multirow[b]{2}{*}{$\begin{array}{l}\text { Costs per } \\
\text { unit }(€)\end{array}$} & \multicolumn{2}{|c|}{ Resources } & \multicolumn{2}{|c|}{$\begin{array}{c}\text { Costs } \\
(\epsilon, \text { standard error of mean) }\end{array}$} \\
\hline & & $\begin{array}{c}\text { DALK } \\
(n=28)\end{array}$ & $\begin{array}{c}\text { PK } \\
(n=25)\end{array}$ & $\begin{array}{l}\text { DALK } \\
(n=28)\end{array}$ & $\begin{array}{c}\text { PK } \\
(n=25)\end{array}$ \\
\hline \multicolumn{6}{|l|}{ Preoperative costs } \\
\hline BIS foundation services & 3599/cornea & 1 & 1 & 3599 & 3599 \\
\hline Out-patient visits & 27/visit & 1 & 0.6 & $28(4)$ & $16(3)$ \\
\hline Diagnostic procedures & variable & 1 & 0.9 & $18(4)$ & $17(4)$ \\
\hline Subtotal & & & & 3645 & 3632 \\
\hline \multicolumn{6}{|l|}{ Surgical procedures } \\
\hline \multicolumn{6}{|l|}{ Transplantation } \\
\hline Operating room costs & $9.50 /$ minute & 148 & 115 & $1407(51)$ & $1096(68)$ \\
\hline Ophthalmology costs & $6.20 /$ minute & 117 & 85 & 725 (29) & $529(39)$ \\
\hline \multicolumn{6}{|l|}{ Additional procedures } \\
\hline Operating room costs & $9.50 /$ minute & 20 & 4 & $193(63)$ & $39(23)$ \\
\hline Ophthalmology costs & $6.20 /$ minute & 17 & 2 & $107(31)$ & $13(7)$ \\
\hline Subtotal & & & & 2432 & 1677 \\
\hline \multicolumn{6}{|l|}{ Hospitalization } \\
\hline Day care & 229/day & 0.3 & 0.2 & $71(20)$ & $49(19)$ \\
\hline Admission & 248/day & 3.4 & 2.7 & $848(113)$ & $679(55)$ \\
\hline Laboratory tests & variable & 0.9 & 0.9 & $48(7)$ & $32(5)$ \\
\hline Subtotal & & & & 967 & 760 \\
\hline \multicolumn{6}{|l|}{ Follow-up visits } \\
\hline Outpatient visits & 27/visit & 11.5 & 10.2 & $311(18)$ & $276(18)$ \\
\hline Diagnostic procedures & variable & 9.6 & 6.8 & $212(24)$ & $167(22)$ \\
\hline Subtotal & & & & 523 & 443 \\
\hline Postoperative drugs & 10/bottle & 4 & 4 & 40 & 40 \\
\hline Total costs $(€)$ & & & & $7607(232)$ & $6552(128)$ \\
\hline
\end{tabular}

BIS = Bio Implant Services; DALK = deep anterior lamellar keratoplasty; PK = penetrating keratoplasty. 


\section{Cost-effectiveness}

Costs per clinically improved patient on the 25-item National Eye Institute Visual Functioning Questionnaire. The ICER is $€ 9977$ (US\$13 768) per clinically improved patient on the NEI VFQ-25. In Figure 3, the cost-effectiveness plane of the bootstrapped results is shown to estimate the uncertainty in costs and effects. Ninety-four percent of the incremental cost-effectiveness pairs is located in the northeast quadrant (more costly, more effective), whereas $6 \%$ is situated in the inferior northwest quadrant. The cost-effectiveness acceptability curve (Figure 4) shows that the probability of DALK being cost-effective ranges from $4 \%$ at a ceiling ratio of $€ 5000$ (US\$6900) to $79 \%$ at a ceiling ratio of $€ 20000$ (US\$27 600).

Costs per patient with an endothelial cell loss of maximally $20 \%$ within the first year. The ICER is $€ 6900$ (US\$9522) per patient with an endothelial cell loss of maximally $20 \%$ within the first year. The cost-effectiveness plane of the bootstrapped results shows that almost all incremental cost-effectiveness pairs are located in the northeast quadrant (Figure 5). In Figure 6, the cost-effectiveness acceptability curve shows that the probability that DALK is cost effective is $15 \%$ at a ceiling ratio of $€ 5000$ (US\$6900), increasing to $96 \%$ at a ceiling ratio of $€ 20000$ (US\$27 600).

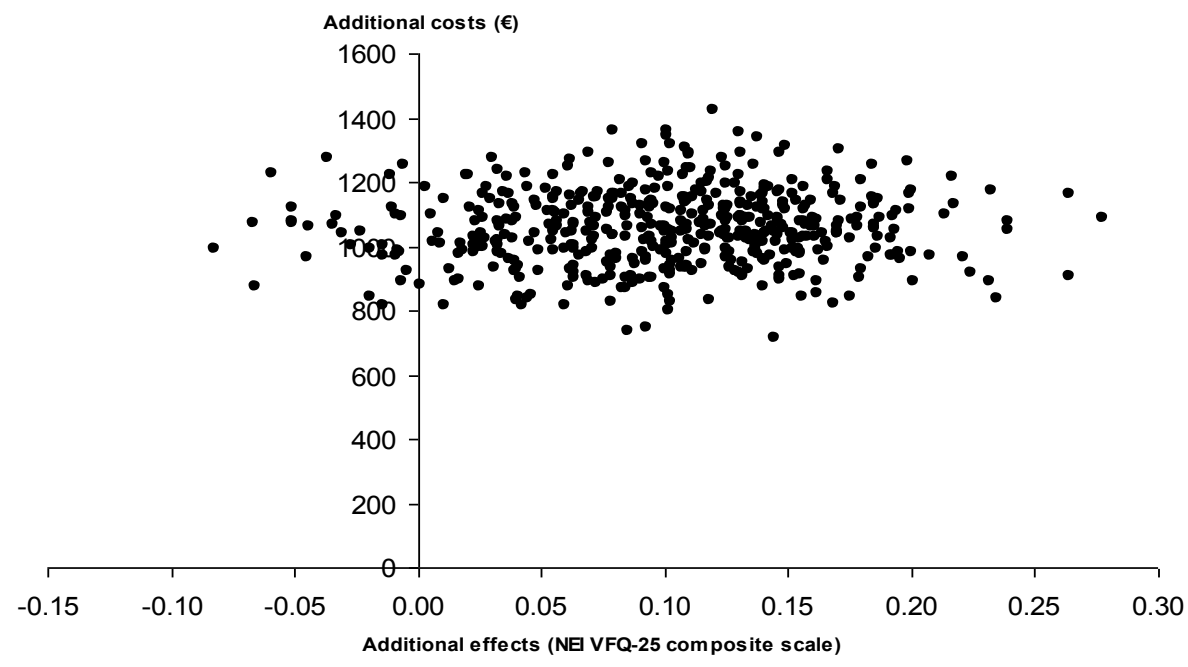

FIGURE 3. Scatterplot showing cost-effectiveness plane for the costs per clinically improved patient on the 25-item National Eye Institute Visual Functioning Questionnaire (NEI VFQ-25) for deep anterior lamellar keratoplasty (DALK) versus penetrating keratoplasty (PK). Costs are plotted on the vertical axis and effects are plotted on the horizontal axis, so an incremental cost-effectiveness pair in the northeast quadrant means that DALK is more effective and more costly than PK for that pair. 


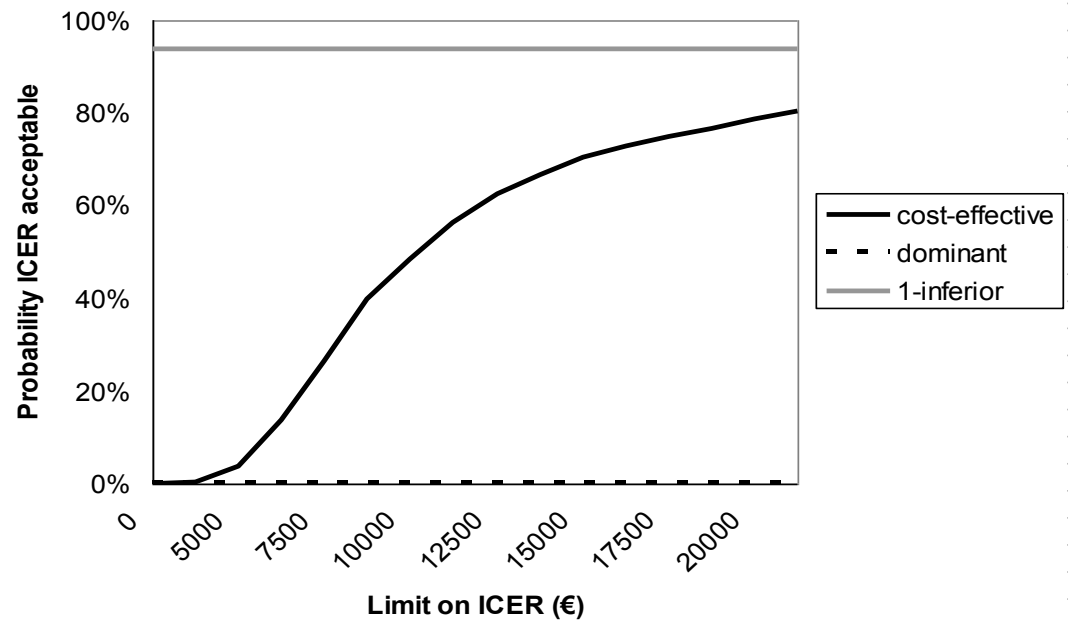

FIGURE 4. Cost-effectiveness acceptability curve for the costs per clinically improved patient on the 25 -item National Eye Institute Visual Functioning Questionnaire composite score for deep anterior lamellar keratoplasty (DALK) versus penetrating keratoplasty (PK). The curve shows the probability (vertical axis) that DALK is cost-effective compared with PK over a range of values for the maximum acceptable ceiling ratio (horizontal axis). ICER = incremental cost-effectiveness ratio.

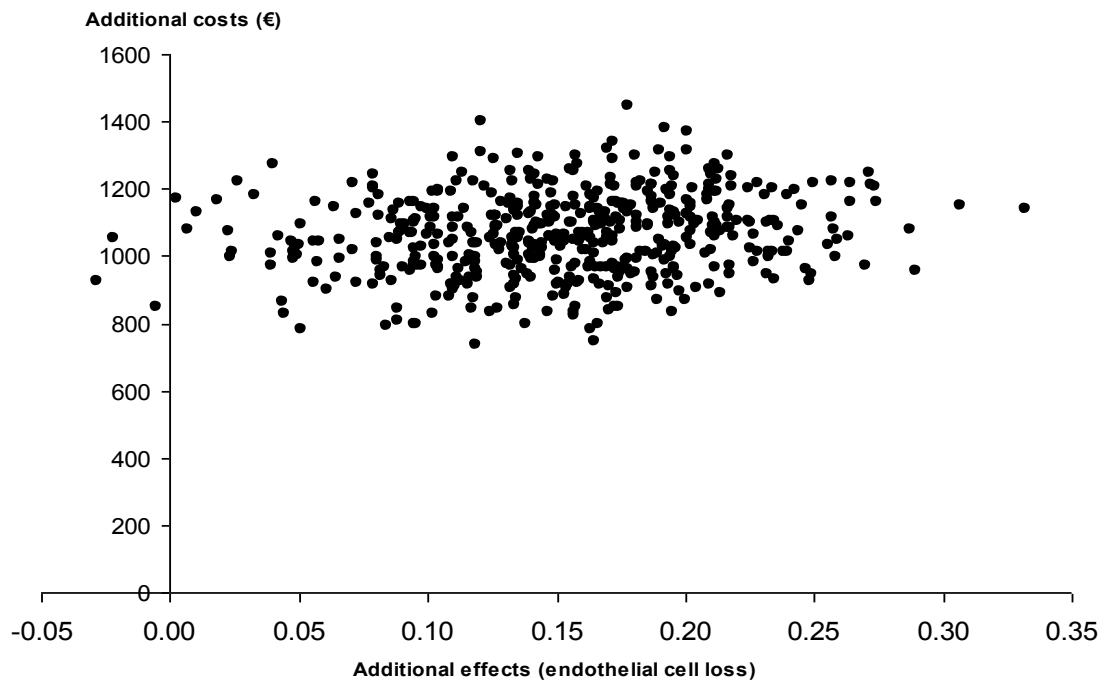

FIGURE 5. Scatterplot showing cost-effectiveness plane for the costs per patient with an endothelial cell loss of maximally $20 \%$ within the first year for deep anterior lamellar keratoplasty (DALK) versus penetrating keratoplasty (PK). Costs are plotted on the vertical axis and effects are plotted on the horizontal axis, so an incremental cost-effectiveness pair in the northeast quadrant means that DALK is more effective and more costly than PK for that pair. 


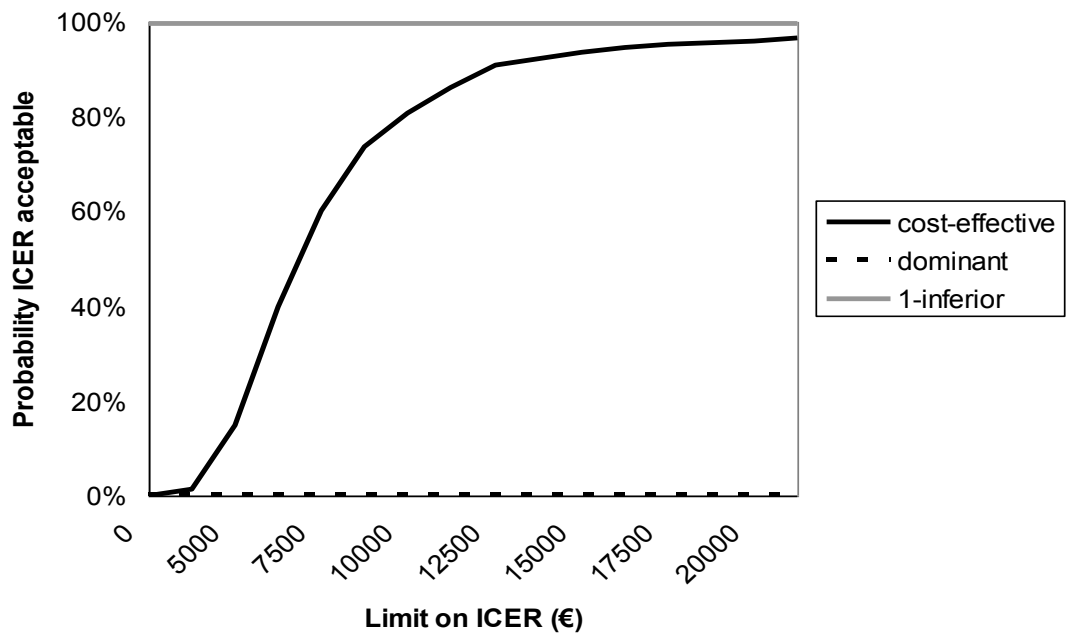

FIGURE 6. Cost-effectiveness acceptability curve for the costs per patient with an endothelial cell loss of maximally $20 \%$ within the first year for deep anterior lamellar keratoplasty (DALK) versus penetrating keratoplasty (PK). The curve shows the probability (vertical axis) that DALK is costeffective compared with PK over a range of values for the maximum acceptable ceiling ratio (horizontal axis). ICER $=$ incremental cost-effectiveness ratio.

\section{Secondary analyses}

Table 5 shows the results of the primary and secondary analyses. Costs per clinically improved patient ranged from $€ 7917$ (US\$10 925) in the secondary analysis of exclusion of patients with perforation of the Descemet membrane to $€ 18095$ (US\$24 971) in the secondary analysis of a 5-point change on the NEI VFQ-25, compared with €9977 (US\$13 768) in the primary analysis. The probability that DALK is cost effective at a maximum ceiling ratio of $€ 20000$ (US\$27 600) ranged from $55 \%$ to $88 \%$, compared with $79 \%$ in the primary analysis. Costs per patient with an endothelial cell loss of maximally $20 \%$ within the first year ranged from $€ 5250$ (US\$7245) in the secondary analysis of exclusion of patients with perforation of the Descemet membrane to $€ 8975$ (US\$12 385) in the secondary analysis of standardized Dutch unit prices, compared with $€ 6900$ (US\$9522) in the primary analysis. The probability that DALK is cost effective at a maximum ceiling ratio of $€ 20000$ (US\$27 600) ranged from $91 \%$ to $100 \%$, compared with $96 \%$ in the primary analysis. These results of the secondary analyses show that the main costeffectiveness data are robust. 
Table 5: Results of primary and secondary analyses for DALK versus PK

\begin{tabular}{|c|c|c|c|c|c|c|}
\hline \multirow[t]{2}{*}{ Analysis } & \multicolumn{2}{|c|}{ Costs $(€)$} & \multicolumn{2}{|c|}{ Effects $(€)$} & \multirow[t]{2}{*}{$\operatorname{ICER}(€)$} & \multirow{2}{*}{$\begin{array}{l}\text { Probability DALK } \\
\text { cost-effective at } \\
\text { a ceiling ratio of } \\
€ 5000 / € 10000 / \\
€ 20000(\%)\end{array}$} \\
\hline & DALK & PK & DALK & PK & & \\
\hline \multicolumn{7}{|l|}{ Primary analysis } \\
\hline Clinically improved on the NEI VFQ-25 & 7607 & 6552 & 0.59 & 0.48 & 9977 & $4 / 49$ / 79 \\
\hline Endothelial cell loss of maximally $20 \%$ & 7607 & 6552 & 0.39 & 0.24 & 6900 & 15 / $81 / 96$ \\
\hline \multicolumn{7}{|c|}{ Exclusion of patients with perforation of the Descemet's membrane } \\
\hline Clinically improved on the NEI VFQ-25 & 7502 & 6552 & 0.60 & 0.48 & 7917 & 14 / 66 / 88 \\
\hline Endothelial cell loss of maximally $20 \%$ & 7502 & 6552 & 0.42 & 0.24 & 5250 & $46 / 95 / 100$ \\
\hline \multicolumn{7}{|l|}{ Standardized Dutch unit prices } \\
\hline Clinically improved on the NEI VFQ-25 & 9465 & 8093 & 0.59 & 0.48 & 12977 & $0 / 29 / 74$ \\
\hline Endothelial cell loss of maximally $20 \%$ & 9465 & 8093 & 0.39 & 0.24 & 8975 & $2 / 59$ / 91 \\
\hline \multicolumn{7}{|l|}{ 5-point change on NEI VFQ-25 } \\
\hline Clinically improved on the NEI VFQ-25 & 7607 & 6552 & 0.71 & 0.66 & 18095 & $0 / 19 / 55$ \\
\hline \multicolumn{7}{|l|}{ As treated } \\
\hline Clinically improved on the NEI VFQ-25 & 7654 & 6692 & 0.58 & 0.50 & 11645 & $5 / 43 / 74$ \\
\hline Endothelial cell loss of maximally $20 \%$ & 7654 & 6692 & 0.39 & 0.27 & 7718 & $13 / 72$ / 92 \\
\hline
\end{tabular}

DALK = deep anterior lamellar keratoplasty; NEI VFQ-25 = 25-item National Eye Institute Visual Functioning Questionnaire; $\mathrm{PK}=$ penetrating keratoplasty.

\section{DISCUSSION}

This economic evaluation comparing DALK with PK demonstrated that DALK was more costly than PK. However, change scores on the NEI VFQ-25 composite score were higher in the DALK group, resulting in an ICER of $€ 9977$ (US\$13 768) per clinically improved patient on the NEI VFQ-25. Furthermore, endothelial cell loss was lower in DALK patients as compared with PK patients, resulting in an ICER of $€ 6900$ (US\$9522) per patient with an endothelial cell loss of maximally $20 \%$ within the first year. In the additional analysis with patients without perforation of the Descemet membrane, an ICER of $€ 7917$ (US\$10 925) per clinically improved patient on the NEI VFQ-25 and an ICER of $€ 5250$ (US\$7245) per patientwith an endothelial cell loss of maximally $20 \%$ was found.Visual outcomes were comparable between DALK and PK patients.

Cost-effectiveness analysis aims to improve decisions on allocation of resources by policy makers and insurers. To compare 2 treatments, an ICER can be calculated, indicating the additional costs per extra unit of effect. This study shows that DALK costs society $€ 9977$ (US\$13 768) per additional clinically improved patient and €6900 (US\$9522) per additional patient with an endothelial cell loss of maximally $20 \%$ within the first year. 
However, what society is willing to pay for an additional improved patient has not yet been evaluated, and no studies are found that evaluated cost effectiveness using the NEI VFQ-25 or endothelial cell loss as an outcome measure. Therefore, it is difficult to interpret these results. However, compared with the mean total costs per patient, which were $€ 7607$ (US\$10 498) and $€ 6552$ (US\$9042) in the DALK group and the PK group, respectively, the ICERs seem considerably high.

It is known that the cost effectiveness of health technologies may vary between countries caused by so-called transferability factors, such as methodologic characteristics (i.e., medical cost approach), health care system characteristics (i.e., characteristics of evaluated technology), and population characteristics (i.e., case mix). ${ }^{36,37}$ Because this study was conducted in The Netherlands, the results therefore may not be directly transferable to the United States. However, because the study data and methods are presented in a transparent and reproducible way, we believe that simple adaptations may suffice. For example, as in Table 4, volumes of resource use and prices are presented separately, readers are able to use different prices and recalculate costs.

In our study, endothelial cell loss at 12 months after surgery was $24.1 \%$ in the DALK group and $28.1 \%$ in the PK group. The higher endothelial cell loss in PK-treated eyes is in accordance with previous studies. ${ }^{4,9,11,13,18,38}$ The endothelial cell loss in the DALK group is somewhat higher compared with cell loss in other studies ranging from $6 \%$ to $20 \%$ in the first year. ${ }^{4,26-28}$ However, in the DALK group, endothelial cell loss was higher at 6 months compared with 12 months, indicating cell loss stabilization within 12 months. This is in concordance with a study by Shimazaki and associates, who found a stabilization of endothelial cell loss 6 months after a DALK procedure. ${ }^{9}$

A limitation of the study is the follow-up period, which was only 12 months. During the first year, the mean total costs per patient were $€ 7607$ (US\$10 498) in the DALK group and $€ 6552$ (US\$9042) in the PK group. Costs differences were related mainly to the transplantation procedure and the additional surgical procedures. The higher costs regarding the transplantation procedure can be explained by the technically challenging and time-consuming big-bubble technique that is used in the DALK group, which increases the time needed for surgery as compared with PK. It needs to be mentioned that the patients included in this study are among the first treated with DALK in the participating hospitals and that the procedure has a definite learning curve. For example, 3 of the 5 patients in the DALK group who were converted to PK were among the first 5 patients included in the study. Clearly, this increased the surgery time, resulting in higher costs. In addition, this may have increased the time and costs of hospitalization in the DALK group. Furthermore, in the DALK group, more money was spent on additional surgical procedures. This is mainly caused by additional surgery as a result of conversion to $\mathrm{PK}$ and postoperative air injection in the anterior chamber. 
The differences in costs of follow-up visits between DALK and PK can be explained by the higher complication rate in the DALK group. Besides perforations of the Descemet membrane and conversion to $\mathrm{PK}$, more suture reactions and elevated intraocular pressure was found in the DALK group. Further details of the complication profile have been described elsewhere. ${ }^{35}$ Although costs within 1 year after surgery were higher in DALK patients, it could be argued that long-term costs are higher in PK patients because of the higher risk of graft failure and need for retransplantation.

In DALK patients, the preservation of healthy endothelium reduces the incidence of endothelial rejection, which is one of the major reasons for graft failure. Several studies showed a graft failure of $10 \%$ to $38 \%$ at 5 years ${ }^{6,8,13,39-42}$ and $18 \%$ to $37 \%$ at 10 years ${ }^{6,13,43}$ in PK patients. In DALK patients, a graft failure of $3 \%$ after 5 years has been reported. ${ }^{13}$ In a study by Borderie and associates, the predicted graft survival was $41 \%$ at 20 years and $3 \%$ at 30 years for PK patients. ${ }^{13}$ For DALK patients, this was $63 \%$ and $11 \%$, respectively. These results indicate that in the long term, graft survival is higher in DALK patients as compared with PK patients. Therefore, the need for retransplantation will be less in DALK patients as compared with PK patients, which will have a positive effect on the long-term cost effectiveness of DALK.

In addition, the composite score on the NEI VFQ-25 shows a downward trend over time in the PK group between 6 and 12 months, whereas in the DALK group, an increasing trend is displayed (Figure 2). Although a longer follow-up period is needed to evaluate this further, this may indicate that DALK is more effective in the long term.

The results of this study show that DALK is more costly and more effective as compared with PK. Results on theNEI VFQ-25 were in favor of DALK, and endothelial cell loss in DALK patients remained stable after 6 months after surgery, whereas cell loss in PK patients continued. Furthermore, it is shown that DALK procedures performed without perforation of the Descemet membrane were more effective. However, because it is unknown what society is willing to pay for an additional improved patient, the cost effectiveness of DALK within a limited follow-up period of 12 months is unclear. Because cost effectiveness of DALK may improve over time because of lower graft failure, studies with a longer follow-up period are necessary. Furthermore, further surgical improvements to reduce the perforation rate will improve the cost effectiveness of DALK. 


\section{REFERENCES}

1. Pramanik S, Musch DC, Sutphin JE, Farjo AA. Extended long-term outcomes of penetrating keratoplasty for keratoconus. Ophthalmology 2006;113(9):1633-1638.

2. Zadok D, Schwarts S, Marcovich A, et al. Penetrating keratoplasty for keratoconus: long-term results. Cornea 2005; 24(8):959 -961.

3. Anshu A, Parthasarathy A, Mehta JS, Htoon HM, Tan DT. Outcomes of therapeutic deep lamellar keratoplasty and penetrating keratoplasty for advanced infectious keratitis: a comparative study. Ophthalmology 2009;116(4): 615-623.

4. Bahar I, Kaiserman I, Srinivasan S, Ya-Ping J, Slomovic AR, Rootman DS. Comparison of three different techniques of corneal transplantation for keratoconus. Am J Ophthalmol 2008;146(6):905-912.

5. Beckingsale P, Mavrikakis I, AI-Yousuf N, Mavrikakis E, Daya SM. Penetrating keratoplasty: outcomes from a corneal unit compared to national data. Br J Ophthalmol 2006;90(6):728 -731.

6. Thompson RW Jr, Price MO, Bowers PJ, Price FW Jr. Long-term graft survival after penetrating keratoplasty. Ophthalmology 2003;110(7):1396-1402.

7. Patel SV, Hodge DO, Bourne WM. Corneal endothelium and postoperative outcomes 15 years after penetrating keratoplasty. Am J Ophthalmol 2005;139(2):311-319.

8. Lass JH, Gal RL, Dontchev M, et al. Donor age and corneal endothelial cell loss 5 years after successful corneal transplantation. Specular microscopy ancillary study results. Ophthalmology 2008;115(4):627-632.

9. Shimazaki J, Shimmura S, Ishioka M, Tsubota K. Randomized clinical trial of deep lamellar keratoplasty vs penetrating keratoplasty. Am J Ophthalmol 2002;134(2): 159 -165.

10. Sutphin JE, Goins KM, Wagoner MD. Deep anterior lamellar keratoplasty: when should it replace penetrating keratoplasty? Am J Ophthalmol 2009;148(5):629-631.

11. Panda A, Bageshwar LM, Ray M, Singh JP, Kumar A. Deep lamellar keratoplasty versus penetrating keratoplasty for corneal lesions. Cornea 1999;18(2):172-175.

12. Sugita J, Kondo J. Deep lamellar keratoplasty with complete removal of pathological stroma for vision improvement. Br J Ophthalmol 1997;81(3):184-188.

13. Borderie VM, Boelle PY, Touzeau O, Allouch C, Boutboul S, Laroche L. Predicted long-term outcome of corneal transplantation. Ophthalmology 2009;116(12):2354-2360.

14. Sarnicola V, Toro P. Deep anterior lamellar keratoplasty in herpes simplex corneal opacities. Cornea 2010;29(1):60-64.

15. Han DC, Mehta JS, Por YM, Htoon HM, Tan DT. Comparison of outcomes of lamellar keratoplasty and penetrating keratoplasty in keratoconus. Am J Ophthalmol 2009;148(5):744-751.

16. Drummond MF, Sculpher MJ, Torrance GW, O'Brien BJ, Stoddart GL. Methods for the economic evaluation of health care programmes, 3th ed. Oxford: Oxford University Press,2005.

17. Brown MM, Brown GC, Sharma S, Landy J. Health care economic analyses and value-based medicine. Surv Ophthalmol 2003;48(2):204-223.

18. Trimarchi F, Poppi E, Klersy C, Piacentini C. Deep lamellar keratoplasty. Ophthalmologica 2001;215(6):389-393.

19. Ing JJ, Ing HH, Nelson LR, Hodge DO, Bourne WM. Ten-year postoperative results of penetrating keratoplasty. Ophthalmology 1998;105(10):1855-1865.

20. Anwar M, Teichmann KD. Big-bubble technique to bare Descemet's membrane in anterior lamellar keratoplasty. J Cataract Refract Surg 2002;28(3):398-403. 
21. Vajpayee RB, Tyagi J, Sharma N, Kumar N, Jhanji V, Titiyal JS. Deep anterior lamellar keratoplasty by big-bubble technique for treatment corneal stromal opacities. Am J Ophthalmol 2007;143(6):954 $-957$.

22. Mangione CM, Lee PP, Gutierrez PR, Spritzer K, Berry S, Hays RD. Development of the 25-item National Eye Institute Visual Function Questionnaire. Arch Ophthalmol 2001;119(7):1050 -1058.

23. Lindblad AS, Clemons TE. Responsiveness of the National Eye Institute Visual Function Questionnaire to progression to advanced age-related macular degeneration, vision loss, and lens opacity: AREDS report no. 14. Arch Ophthalmol 2005;123(9):1207-1214.

24. Miskala PH, Bass EB, Bressler NM, et al. Surgery for subfoveal choroidal neovascularization in age-related macular degeneration: quality-of-life findings: SST report no. 12. Ophthalmology 2004;111(11):1981-1992.

25. Miskala PH, Hawkins BS, Mangione CM, et al. Responsiveness of the National Eye Institute Visual Function Questionnaire to changes in visual acuity: findings in patients with subfoveal choroidal neovascularization-SST report no. 1. Arch Ophthalmol 2003;121(4):531-539.

26. Fontana L, Parente G, Tassinari G. Clinical outcomes after deep anterior lamellar keratoplasty using the big-bubble technique in patients with keratoconus. Am J Ophthalmol 2007;143(1):117-124.

27. Kawashima M, Kawakita T, Den S, Shimmura S, Tsubota K, Shimazaki J. Comparison of deep lamellar keratoplasty and penetrating keratoplasty for lattice and macular corneal dystrophies. Am J Ophthalmol 2006;142(2):304-309.

28. Morris E, Kirwan JF, Sujatha S, Rostron CK. Corneal endothelial specular microscopy following deep lamellar keratoplasty with lyophilised tissue. Eye (Lond) 1998;12 (Pt 4):619-622.

29. Chung SH, Kim HK, Kim MS. Corneal endothelial cell loss after penetrating keratoplasty in relation to preoperative recipient endothelial cell density. Ophthalmologica 2009; 224(3):194 -198.

30. Bertelmann E, Pleyer U, Rieck P. Risk factors for endothelial cell loss post-keratoplasty. Acta Ophthalmol Scand 2006; 84(6):766 -770.

31. Oostenbrink JB, Bouwmans CAM, Koopmanschap MA, Rutten FFH. Handleiding voor kostenonderzoek: methoden en standaard kostprijzen voor economische evaluaties in de gezondheidszorg. Diemen: College voor Zorgverzekeringen, 2004.

32. Organization for Economic Co-operation and Development. Table 4: PPP and exchange rates. Available at: http://Stats.Oecd.Org/Index.Aspx?Datasetcode=Sna_Table4. Accessed June 1, 2010.

33. Dutch Health Care Insurance Board. Pharmacotherapeutic Compass [in Dutch]. Amstelveen: College voor Zorgverzekeringen, 2008.

34. Barber JA, Thompson SG. Analysis of cost data in randomized trials: an application of the nonparametric bootstrap. Stat Med 2000;19(23):3219 -3236.

35. Cheng YYY, Visser N, Schouten JS, et al. Endothelial cell loss and visual outcome of deep anterior lamellar keratoplasty versus penetrating keratoplasty-a randomized multicenter clinical trial. Ophthalmology 2010. Ophthalmology. 2011 Feb;118(2):302-309.

36. Drummond M, Barbieri M, Cook J, et al. Transferability of economic evaluations across jurisdictions: ISPOR Good Research Practices Task Force report. Value Health 2009; 12(4):409-418.

37. Welte R, Feenstra T, Jager H, Leidl R. A decision chart for assessing and improving the transferability of economic evaluation results between countries. Pharmacoeconomics 2004;22(13):857-876.

38. Krumeich JH, Knulle A, Krumeich BM. [Deep anterior lamellar (DALK) vs. penetrating keratoplasty (PKP): a clinical and statistical analysis]. Klin Monbl Augenheilkd 2008;225(7):637- 648.

39. Gal RL, Dontchev M, Beck RW, et al. The effect of donor age on corneal transplantation outcome results of the cornea donor study. Ophthalmology 2008;115(4):620-626. 
40. Tan DT, Janardhanan P, Zhou H, et al. Penetrating keratoplasty in Asian eyes: the Singapore Corneal Transplant Study. Ophthalmology 2008;115(6):975-982.

41. Williams KA, Esterman AJ, Bartlett C, Holland H, Hornsby NB, Coster DJ. How effective is penetrating corneal transplantation? Factors influencing long-term outcome in multivariate analysis. Transplantation 2006;81(6):896 -901.

42. Sugar A, Tanner JP, Dontchev M, et al. Recipient risk factors for graft failure in the cornea donor study. Ophthalmology 2009;116(6):1023-1028.

43. Inoue K, Kimura C, Amano S, et al. Long-term outcome of systemic cyclosporine treatment following penetrating keratoplasty. Jpn J Ophthalmol 2001;45(4):378 -382. 



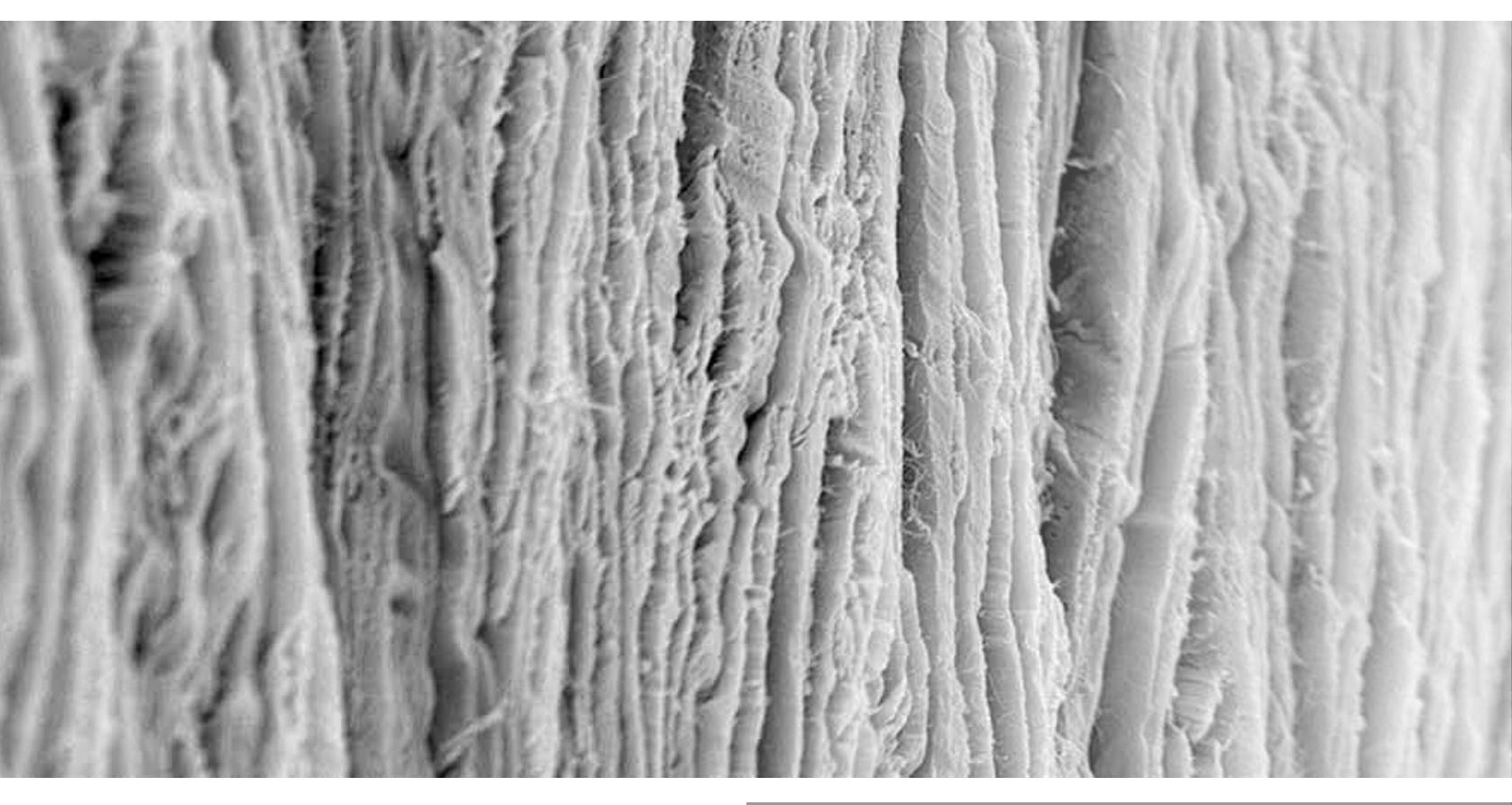




\section{CHAPTER 13}

General Discussion 

In 1906, the first successful human penetrating keratoplasty (PK) was performed by Dr. Eduard Zirm. ${ }^{1}$ Worldwide, human corneas are the most commonly transplanted tissue and most successful tissue transplantation type. Until the last decade, PK has been the golden standard surgical technique for most corneal diseases. Several important innovative techniques and instruments have been introduced with a transformation to selective lamellar keratoplasty.

\section{Penetrating keratoplasty versus deep anterior lamellar keratoplasty}

In about $30 \%$ of keratoplasty cases, the indications are corneal pathologies with a normal endothelium. Among these, keratoconus is the most common indication. ${ }^{2}$ In the past, PK was considered the standard surgical keratoplasty technique. However, endothelial allograft rejection was one of the most important reasons for graft failure in these patients. ${ }^{3}$

Deep anterior lamellar keratoplasty (DALK) aims to replace the recipient epithelium and corneal stroma, leaving Descemet's membrane and the endothelium intact, thus eliminating endothelial allograft rejection. In a comparative study by Borderie et al, the median predicted graft survival was 49.0 years in the DALK group versus 17.3 years in the PK group. ${ }^{4}$ Therefore, the need for re-transplantation will be less in DALK patients as compared to PK patients.

Since endothelial allograft rejection is eliminated in DALK patients, topical corticosteroid treatment can be discontinued earlier than in PK patients, thereby reducing the incidence of glaucoma and/or cataract. ${ }^{5}$ Another advantage of the DALK technique is the prevention of long-term endothelial cell loss. In this thesis, endothelial cell loss after DALK without perforation of Descemet's membrane was $12.9 \%$ and $27.7 \%$ after PK with a follow-up of 12 months. ${ }^{6}$ During the 1 year follow-up of our study, endothelial cell loss did not show evident stabilization after DALK. However, several studies showed stabilization of endothelial cell loss 6 months after a DALK procedure., ${ }^{5}$ A longer follow-up is needed to evaluate this further.

Furthermore, in our study we found a comparable visual outcome between the DALK and PK groups at 6 and 12 months follow-up. ${ }^{6}$ This finding indicates that DALK with Anwar's big-bubble technique is able to achieve such a deep dissection up to the level of Descemet's membrane that it does not lead to visually disturbing interface haze. Although another study did find a significantly better best-corrected visual acuity after DALK, ${ }^{8}$ the majority of studies have reported similar visual outcome values in DALK versus PK eyes. ${ }^{7}$

Our cost-effectiveness analysis showed that DALK is both more expensive but also more effective than a PK. ${ }^{9}$ However, this study was done over a limited follow-up time of 12 months. To evaluate the cost-effectiveness more accurately and whether it will 
improve over time (e.g. due to lower graft failure), studies with a longer follow-up are necessary.

It is known that many countries suffer from a shortage in available and suitable donor corneas. Another important advantage of the DALK technique is that more donor corneas will be available for transplantation, whereas for PK techniques they would have been discarded due to their low endothelial cell density.

Despite these advantages, the number of anterior lamellar keratoplasty techniques performed in the United States remain low compared to PK techniques for keratoconus. ${ }^{2}$ This can be explained by the technical challenges in and surgical learning curve in separating the anterior stromal layer from Descemet's membrane and endothelium.

Furthermore, the main complication of the DALK technique is intraoperative perforation of Descemet's membrane, which has been reported with a range of $4 \%$ to $39 \%{ }^{7}$ There is substantial risk of required conversion to PK during a DALK procedure, so most corneal surgeons tend to order a donor cornea that is suitable for both DALK and PK, except for hospitals with an eye bank who can supply a back-up donor cornea with high endothelial quality (in case of perforation).

More effective use of donor corneas in PK patients can be achieved by further surgical improvements in order to reduce risk of perforation (and thus conversion to PK), and by close collaboration between the surgical team and their eye bank, especially in countries with corneal graft shortages.

\section{Penetrating keratoplasty versus endothelial keratoplasty}

In the USA and Europe, endothelial keratoplasty (EK) techniques constitute about 45$50 \%$ of all transplant procedures. ${ }^{2}$ In the early twentieth century, EK has replaced PK as the golden standard surgical treatment for endothelial cell dysfunction.

Currently, Descemet's stripping automated endothelial keratoplasty (DSAEK) is the most popular EK procedure. The major advantage of DSAEK is that no sutures are required to keep the donor tissue in place, thereby preventing high irregular astigmatism and suture-related events, resulting in faster visual rehabilitation and better wound stability. ${ }^{10}$ Furthermore, DSAEK is a 'closed eye' surgery, which also reduces the risk of expulsive hemorrhage.

Several techniques have been described in order to harvest donor lenticules for EK. The manual lamellar dissection techniques was not very popular due to the amount of technical challenges. ${ }^{11,} 12$ Subsequently, the use of an automated microkeratome and a Femtosecond laser were reported for preparation of the donor lenticule. ${ }^{13}$

This thesis aims to demonstrates the feasibility of Femtosecond laser technology in order to prepare a standardized posterior lamellar lenticule for EK. ${ }^{14}$ The clinical outcome of our randomized study showed lower postoperative astigmatism and absence of wound healing related problems in the group of patients after Femtosecond laser- 
assisted Descemet's stripping endothelial keratoplasty (FS-DSEK). ${ }^{15}$ However, visual acuity is lower as compared to the conventional PK, and the high percentage of endothelial cell loss.

The results of our cost-effectiveness analysis between FS-DSEK, PK and DSAEK showed that FS-DSEK was not cost-effective compared to PK and DSAEK. ${ }^{16}$ DSAEK, on the other hand, was more costly but also more effective. With the increasing amount of cornea banks that are able to prepare lamellar transplant buttons for the various endothelial keratoplasty techniques, it may significantly lower the costs per patients, thereby improving the cost-effectiveness of endothelial keratoplasty.

In 2006, Melles described the technique of Descemet membrane endothelial keratoplasty (DMEK). ${ }^{17}$ The use of DMEK is currently still limited because of major challenges in donor preparation, difficulty to unfold the thin donor lenticule in the anterior chamber, and the high rebubbling rate. ${ }^{18}$ Several studies have shown faster and better visual rehabilitation after DMEK compared to DSAEK. ${ }^{18-20}$ Thickness of the donor lenticule is correlated with better visual acuity. The study of Neff et al showed that a thinner endothelial graft $(\leq 131 \mu \mathrm{m})$ is correlated with a significantly better postoperative visual acuity compared to a thicker endothelial graft $(\geq 131 \mu \mathrm{m}) .{ }^{21}$ Busin et al introduced the ultrathin (UT-) DSAEK technique to prepare a thin donor lenticule $(<130 \mu \mathrm{m})$ with a standardized method. ${ }^{22}$ UT-DSAEK visual outcomes were comparable with reported DMEK studies and with less surgical challenges than DMEK. ${ }^{18,23}$ A study by Dickman et al. found a correlation between a thicker donor graft and greater asymmetry of the posterior corneal surface, which associated with higher posterior corneal higher-order aberrations (HOAs). ${ }^{24}$ This means that thinner grafts result in significantly less posterior corneal (HOAs), probably resulting in higher visual acuity. ${ }^{25}$ Another benefit of a thinner donor graft is reduced allograft rejection compared to DSAEK or PK. ${ }^{26}$

Further randomized clinical research is needed to compare the clinical results of different types of EK (DSAEK, UT-DSAEK and DMEK), and longer-term follow-up is required to analyze the graft survival. 


\section{REFERENCES}

1. Zirm EK. Eine erfolgreiche totale Keratoplastik (A successful total keratoplasty). 1906. Refract Corneal Surg 1989;5(4):258-261.

2. 2012 Eye Banking Statistical Report Eye Bank Association of America. www.restoresight.org

3. Ing JJ, Ing HH, Nelson LR, Hodge DO, Bourne WM. Ten-year postoperative results of penetrating keratoplasty. Ophthalmology 1998;105(10):1855-1865.

4. Borderie VM, Sandali O, Bullet J, Gaujoux T, Touzeau O, Laroche L. Long-term results of deep anterior lamellar versus penetrating keratoplasty. Ophthalmology 2012;119(2):249-255.

5. Shimazaki J, Shimmura S, Ishioka M, Tsubota K. Randomized clinical trial of deep lamellar keratoplasty vs penetrating keratoplasty. Am J Ophthalmol 2002;134(2):159-165.

6. Cheng YY, Visser N, Schouten JS, et al. Endothelial cell loss and visual outcome of deep anterior lamellar keratoplasty versus penetrating keratoplasty: a randomized multicenter clinical trial. Ophthalmology 2011;118(2):302-309.

7. Reinhart WJ, Musch DC, Jacobs DS, Lee WB, Kaufman SC, Shtein RM. Deep anterior lamellar keratoplasty as an alternative to penetrating keratoplasty a report by the american academy of ophthalmology. Ophthalmology 2011;118(1):209-218.

8. Anshu A, Parthasarathy A, Mehta JS, Htoon HM, Tan DT. Outcomes of therapeutic deep lamellar keratoplasty and penetrating keratoplasty for advanced infectious keratitis: a comparative study. Ophthalmology 2009;116(4):615-623.

9. van den Biggelaar FJ, Cheng YY, Nuijts RM, et al. Economic evaluation of deep anterior lamellar keratoplasty versus penetrating keratoplasty in The Netherlands. Am J Ophthalmol 2011;151(3):449459 e442.

10. Anshu A, Price MO, Tan DT, Price FW, Jr. Endothelial keratoplasty: a revolution in evolution. Surv Ophthalmol 2012;57(3):236-252.

11. Melles GR, Eggink FA, Lander F, et al. A surgical technique for posterior lamellar keratoplasty. Cornea 1998;17(6):618-626.

12. Terry MA, Ousley PJ. Deep lamellar endothelial keratoplasty in the first United States patients: early clinical results. Cornea 2001;20(3):239-243.

13. Gorovoy MS. Descemet-stripping automated endothelial keratoplasty. Cornea 2006;25(8):886-889.

14. Cheng YY, Pels E, Nuijts RM. Femtosecond-laser-assisted Descemet's stripping endothelial keratoplasty. J Cataract Refract Surg 2007;33(1):152-155.

15. Cheng YY, Schouten JS, Tahzib NG, et al. Efficacy and safety of femtosecond laser-assisted corneal endothelial keratoplasty: a randomized multicenter clinical trial. Transplantation 2009;88(11):12941302.

16. van den Biggelaar FJ, Cheng YY, Nuijts RM, et al. Economic evaluation of endothelial keratoplasty techniques and penetrating keratoplasty in the Netherlands. Am J Ophthalmol 2012;154(2):272281 e272.

17. Melles GR, Ong TS, Ververs B, van der Wees J. Descemet membrane endothelial keratoplasty (DMEK). Cornea 2006;25(8):987-990.

18. Tourtas T, Laaser K, Bachmann BO, Cursiefen C, Kruse FE. Descemet membrane endothelial keratoplasty versus descemet stripping automated endothelial keratoplasty. Am J Ophthalmol 2012;153(6):1082-1090 e1082.

19. Dirisamer M, Ham L, Dapena I, et al. Efficacy of descemet membrane endothelial keratoplasty: clinical outcome of 200 consecutive cases after a learning curve of 25 cases. Arch Ophthalmol 2011;129(11):1435-1443. 
20. Goldich Y, Showail M, Avni-Zauberman N, et al. Contralateral eye comparison of descemet membrane endothelial keratoplasty and descemet stripping automated endothelial keratoplasty. Am J Ophthalmol 2015;159(1):155-159 e151.

21. Neff KD, Biber JM, Holland EJ. Comparison of central corneal graft thickness to visual acuity outcomes in endothelial keratoplasty. Cornea 2011;30(4):388-391.

22. Busin M, Patel AK, Scorcia V, Ponzin D. Microkeratome-assisted preparation of ultrathin grafts for descemet stripping automated endothelial keratoplasty. Invest Ophthalmol Vis Sci 2012;53(1):521524.

23. Busin M, Madi S, Santorum P, Scorcia V, Beltz J. Ultrathin descemet's stripping automated endothelial keratoplasty with the microkeratome double-pass technique: two-year outcomes. Ophthalmology 2013;120(6):1186-1194.

24. Dickman MM, Cheng YY, Berendschot TT, van den Biggelaar FJ, Nuijts RM. Effects of graft thickness and asymmetry on visual gain and aberrations after descemet stripping automated endothelial keratoplasty. JAMA ophthalmology 2013;131(6):737-744.

25. Rudolph M, Laaser K, Bachmann BO, Cursiefen C, Epstein D, Kruse FE. Corneal higher-order aberrations after Descemet's membrane endothelial keratoplasty. Ophthalmology 2012;119(3):528-535.

26. Anshu A, Price MO, Price FW, Jr. Risk of corneal transplant rejection significantly reduced with Descemet's membrane endothelial keratoplasty. Ophthalmology 2012;119(3):536-540. 


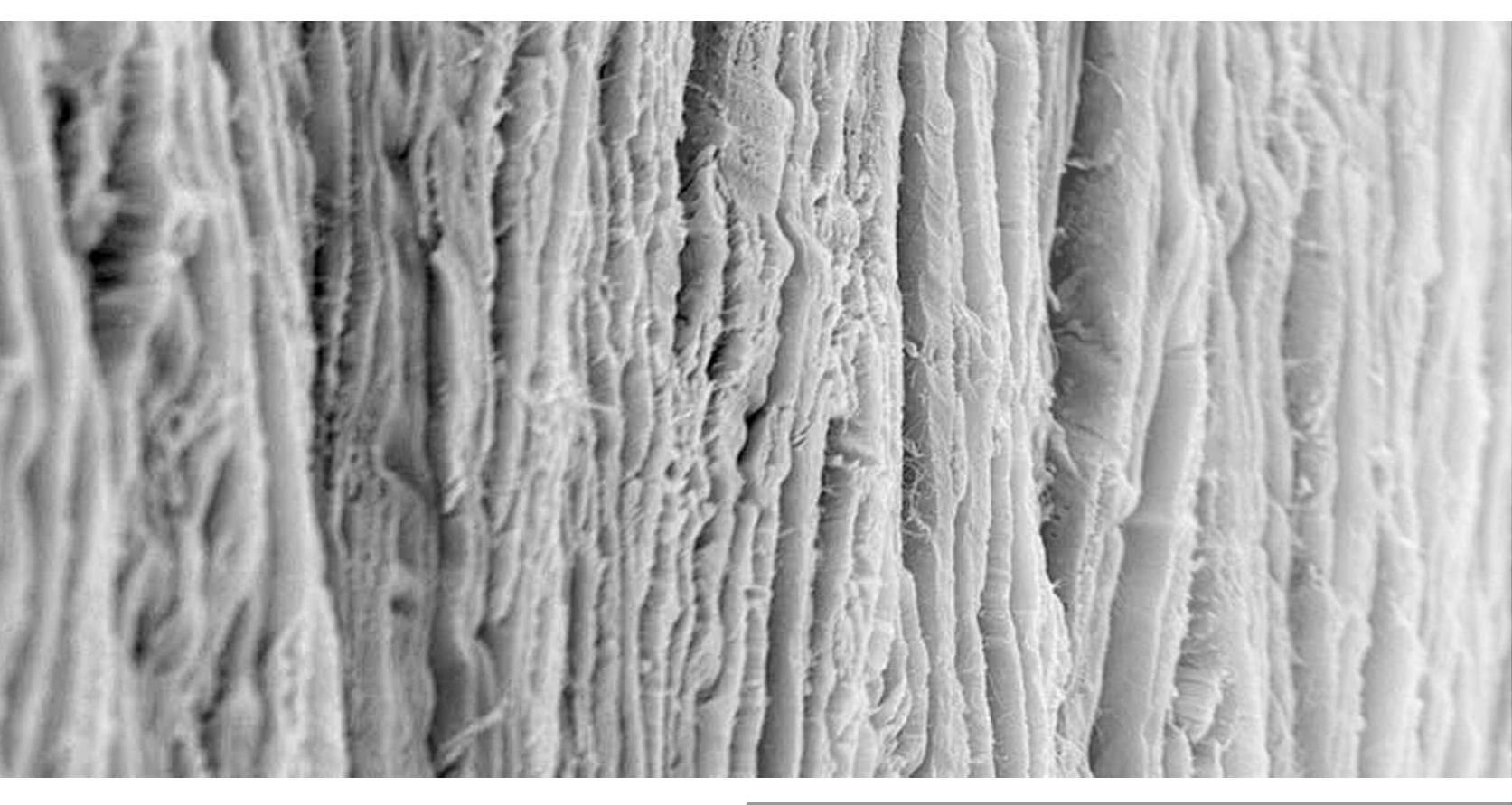


CHAPTER 14

Valorization 

Knowledge valorization refers to implementation of scientific knowledge for social or economic use or by making knowledge for new products, services, system and research.

For many years, full thickness penetrating keratoplasty (PK) was the gold standard treatment for various corneal diseases. In PK, the recipient full-thickness corneal tissue is replaced with full-thickness donor corneal tissue. The goals of PK were visual rehabilitation, tectonic support, reduction of pain, prevention of infection and cosmetic restoration. The leading indications were keratoconus, pseudophakic bullous keratopathy, Fuchs' endothelial dystrophy and regrafts. Other less common indications were post-infectious scarring, diverse corneal dystrophies, perforating corneal injury or chemical burns. ${ }^{1}$

During the years 1998-2001 (prior to starting this thesis research) the percentage of donated eye tissue in the Netherlands issued for corneal grafting decreased from $50.8 \%$ (1998) to 35.2\% (2001). In 2001 the Cornea Bank N.O.R.I. Amsterdam processed 2955 donor eyes of which 1040 were actually grafted. The major reasons for the discard rate of $64.8 \%$ of donated eye tissue were abnormalities of the anterior corneal stroma and a decreased vitality of the corneal endothelium. The shortage of donor tissue had resulted in an average waiting time for corneal transplantation of 6 months.

Lamellar corneal transplantation techniques that only transplant the anterior side of the cornea (deep anterior lamellar keratoplasty, DALK) or the posterior side (endothelial keratoplasty, EK) may use previously discarded tissue by transplantation of only the healthy part of the donated corneal tissue. The use of DALK and EK may lead to a more efficient use of donor material and could theoretically decrease the discard rate of donor tissue and shorten the waiting time. In the current thesis, we studied the clinical outcomes of innovative lamellar corneal transplantation surgery versus PK in a randomized multicenter clinical trial. Further, economic evaluation had been performed between lamellar keratoplasty (DALK and EK) versus PK.

In this chapter of valorization, we discuss the practical implications of the presented studies and the impact at the social and economic level.

In about $30 \%$ of keratoplasty cases, the indications are corneal pathologies with a normal endothelium. The most common indication is keratoconus. Endothelial allograft rejection was one of the most important reasons for graft failure in these patients. The main clinical aim to develop DALK was to preserve the endothelial layer, and to leave Descemet's membrane and the endothelium intact, thus eliminating endothelial allograft rejection. In the study of Borderie et al. the median predicted graft survival was 
49.0 years in the DALK group and 17.3 years in the PK group. ${ }^{2}$ Therefore, the need for re-transplantation will be less in DALK patients as compared to PK patients.

In this thesis the mean age of DALK and PK group is 43 years, and the most common indication for DALK is keratoconus. In these young patients visual impairment has a impact on their social and financial life since they are not able to perform the normal daily working activities. Our cost-effectiveness analysis showed that during the first year, the mean total costs per patient were $€ 7607$ in the DALK group and $€ 6552$ in the PK group. ${ }^{3}$ Costs differences were related mainly to the transplantation procedure and the additional surgical procedures. The higher costs regarding the transplantation procedure can be explained by the technically challenging and time-consuming big-bubble technique that is used in the DALK group, which increases the time needed for surgery as compared with PK. DALK is both more expensive but also more effective than a PK procedure. However, this study was done with a follow-up of 1 year, and it could be argued that long-term costs are higher in PK patients because of higher risk of graft failure and need for re-transplantation.

The main complication of the DALK technique is intraoperative rupture of Descemet's membrane, which required conversion to PK during the DALK procedure. This means that most corneal surgeons will order a donor cornea that is suitable for both DALK and PK. So the concept of using a donor cornea with low endothelial cell density is not widely accepted. More effective use of donor corneas in PK patients can be achieved by further surgical improvements in order to reduce risk of perforation.

In our study we showed that visual acuity was improved after DALK or PK procedure, but the costs were also very high. In cases of keratoconus, which is the most common indication for surgery, corneal crosslinking treatment had been described to stop the progression of the keratoconus. A randomized clinical trial with 3 years follow-up showed that corneal crosslinking was effective to stabilize the progression. ${ }^{4}$ In 2014 , the Dutch Zorginstituut Nederland (ZiNL) decided that epi-off corneal treatment is in line with current standards of care and science and therefore is a treatment option for progressive keratoconus and should be reimbursed by all health insurances.

In the Netherlands, the cost of corneal crosslinking is around $€ 1500$ which is much lower than the cost of corneal transplantation. Long term analysis is needed to determine whether corneal crosslinking will be capable to decrease the need for corneal transplantation in keratoconus patients. Further, it is important for ophthalmologist, optician or eye care professional to diagnose progressive keratoconus in an early stage and to refer patients timely for corneal crosslinking. 
For many years , PK has been the gold-standard technique for corneal transplantation resulting from endothelial disease or endothelial dysfunction. In recent years, in the USA and Europe, EK constitutes for about $45-50 \%$ of all transplant procedures. The major advantage of EK is that no sutures are required to keep the donor tissue in place, thereby preventing high irregular astigmatism and suture related events, resulting in faster visual rehabilitation and better wound stability. ${ }^{5}$

In EK, different techniques have been developed for the preparation of the donor cornea. Several techniques have been described in order to harvest donor lenticules for EK. The manual lamellar dissection techniques was not very popular due to the amount of technical challenges. Subsequently, the use of an automated microkeratome and a Femtosecond laser were reported for preparation of the donor lenticule. ${ }^{6}$

In our study, we showed the feasibility of the Femtosecond laser to prepare a standardized posterior lamellar lenticule for EK. The clinical outcome of our randomized study showed lower postoperative astigmatism and absence of wound healing related problems in the group of patients after Femtosecond laser-assisted Descemet's stripping endothelial keratoplasty (FS-DSEK). ${ }^{7}$ However, visual acuity is lower as compared to the conventional PK, and there was a high percentage of endothelial cell loss.

The results of our cost-effectiveness analysis between FS-DSEK, PK and Descemet's stripping automated endothelial keratoplasty (DSAEK) showed that FS-DSEK was not cost-effective compared to PK and DSAEK. ${ }^{8}$ DSAEK, on the other hand, was more costly but also more effective. The costs within 1 year follow-up were higher in EK patients, but it could be argued that long-term costs are lower and long-term effects are higher as compared to $\mathrm{PK}$, because of the effects of suture removal in the PK group and a reduced risk of complications in the EK groups, with fewer hospital visits and emergency surgical interventions to be expected. However, long-term follow-up is needed to evaluate this potentially positive effect on the long-term cost-effectiveness of EK.

Due to the lower visual outcomes and higher costs of FS-DSEK, the Femtoscond laser technology is not widely use for the preparation of donor lenticules for EK. Currently, DSAEK is the most popular EK procedure and the donor lenticules were prepared with an automated microkeratome. In the beginning the donor lenticules were prepared by the surgeon in the operating room during the surgery. Recently, cornea banks in the Netherlands are able to supply pre-cut donor cornea for DSAEK or Descemet's membrane endothelial keratoplasty (DMEK). This can significantly lower the mean costs per patients for the preparation of the lamellar disks. Further, it will avoid the risk of tissue 
loss during the stromal separation step in the surgery room, and the time needed for surgery has also decreased by approximately fifty percent.

With the automated microkeratome placed at the cornea bank it is now possible to use donor corneas with abnormalities of the anterior stroma and good corneal endothelium for EK procedures. This can lead to more donor corneas available for transplantation, a reduction of discard rate, and a shorter waiting list.

Several studies have shown that thickness of the donor lenticule is correlated with better visual acuity. ${ }^{9}{ }^{10}$ In the Netherlands, a randomized multicenter study is now evaluating the clinical outcomes between ultrathin (UT)-DSAEK and DSAEK. The preliminary results showed that the visual outcomes are significantly better in the UT-DSAEK group (personal communication with R.M.M.A. Nuijts, MD, PhD). Further, several studies have shown faster and better visual rehabilitation after DMEK compared to DSAEK. ${ }^{11}$ As far as we know, there is no randomized clinical trial that has evaluated the clinical outcome of DMEK versus UT-DSAEK. The DMEK procedure is at this moment not accepted as standard treatment by ZiNL because it is not yet in line with current standards of care and science. Further randomized clinical research is needed to compare DMEK with UTDSAEK, and long-term follow up is required to analyze graft survival.

In summary, this thesis presents the clinical outcomes and cost effectiveness of lamellar keratoplasty with the golden standard treatment PK. DALK is a promising surgical technique, but further surgical improvements are needed to be widely accepted. In cases of endothelial dysfunction, the EK procedure is now the first choice of surgical treatment. Nowadays, cornea banks in the Netherlands can provide pre-cut donor corneas and donor cornea with good corneal endothelium and abnormality of the anterior corneal stroma can now be used for EK procedure. 


\section{REFERENCES}

1. Patel SV, Hodge DO, Bourne WM. Corneal endothelium and postoperative outcomes 15 years after penetrating keratoplasty. Am J Ophthalmol 2005;139(2):311-319.

2. Borderie VM, Sandali O, Bullet J, Gaujoux T, Touzeau O, Laroche L. Long-term results of deep anterior lamellar versus penetrating keratoplasty. Ophthalmology 2012;119(2):249-255.

3. van den Biggelaar FJ, Cheng YY, Nuijts RM, et al. Economic evaluation of deep anterior lamellar keratoplasty versus penetrating keratoplasty in The Netherlands. Am J Ophthalmol 2011;151(3):449459 e442.

4. Wittig-Silva C, Chan E, Islam FM, Wu T, Whiting M, Snibson GR. A randomized, controlled trial of corneal collagen cross-linking in progressive keratoconus: three-year results. Ophthalmology 2014;121(4):812-821.

5. Anshu A, Price MO, Tan DT, Price FW, Jr. Endothelial keratoplasty: a revolution in evolution. Surv Ophthalmol 2012;57(3):236-252.

6. Gorovoy MS. Descemet-stripping automated endothelial keratoplasty. Cornea 2006;25(8):886-889.

7. Cheng YY, Schouten JS, Tahzib NG, et al. Efficacy and safety of femtosecond laser-assisted corneal endothelial keratoplasty: a randomized multicenter clinical trial. Transplantation 2009;88(11):12941302.

8. van den Biggelaar FJ, Cheng YY, Nuijts RM, et al. Economic evaluation of endothelial keratoplasty techniques and penetrating keratoplasty in the Netherlands. Am J Ophthalmol 2012;154(2):272281 e272.

9. Neff KD, Biber JM, Holland EJ. Comparison of central corneal graft thickness to visual acuity outcomes in endothelial keratoplasty. Cornea 2011;30(4):388-391.

10. Dickman MM, Cheng YY, Berendschot TT, van den Biggelaar FJ, Nuijts RM. Effects of graft thickness and asymmetry on visual gain and aberrations after descemet stripping automated endothelial keratoplasty. JAMA ophthalmology 2013;131(6):737-744.

11. Goldich Y, Showail M, Avni-Zauberman N, et al. Contralateral eye comparison of descemet membrane endothelial keratoplasty and descemet stripping automated endothelial keratoplasty. Am J Ophthalmol 2015;159(1):155-159 e151. 


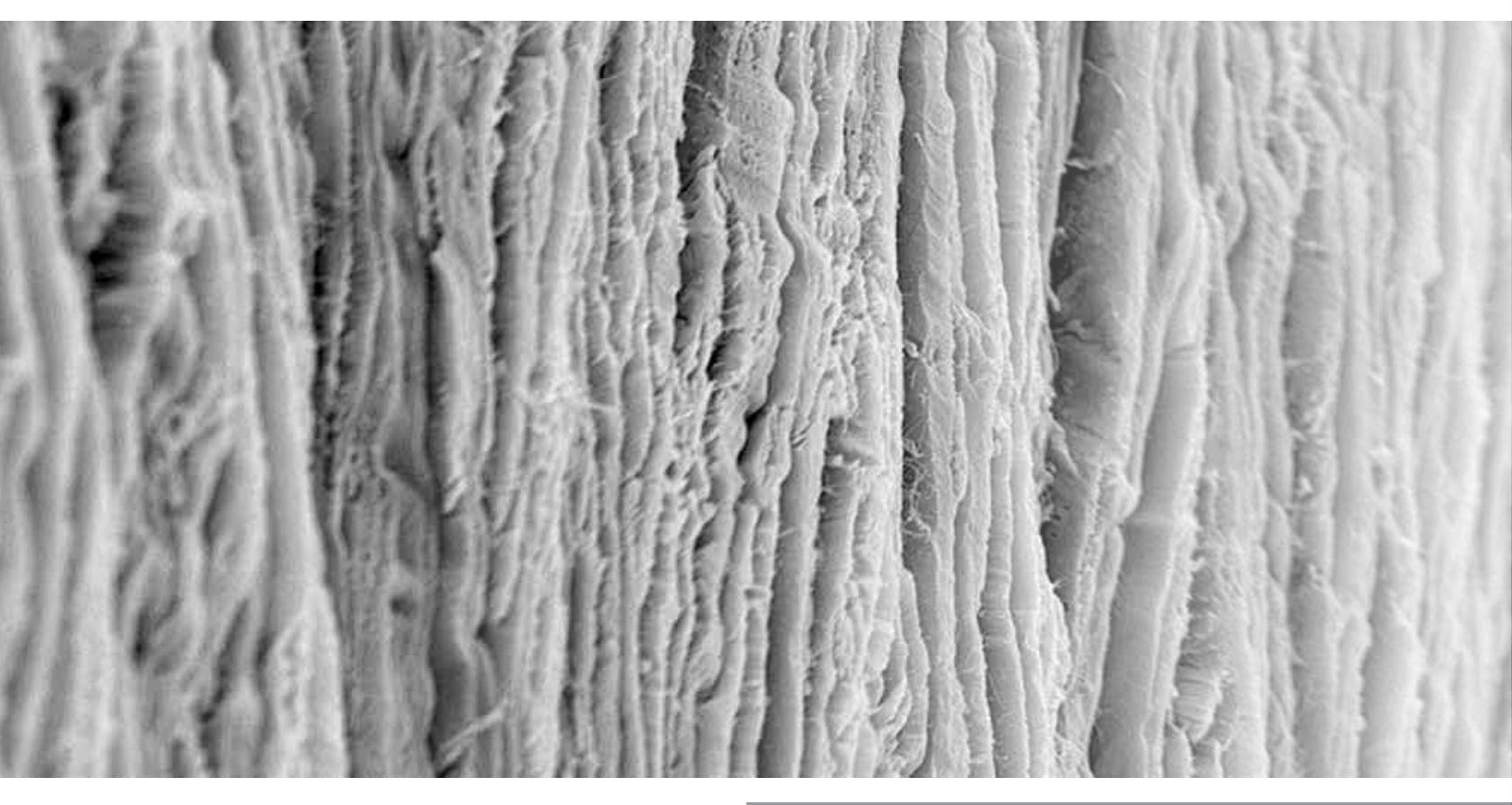




\section{Summary}



In this thesis the clinical outcome of femtosecond laser-assisted Descemet's stripping endothelial keratoplasty (FS-DSEK) and deep anterior lamellar keratoplasty (DALK) versus penetrating keratoplasty (PK) is described and the application of the femtosecond laser in corneal transplantation surgery is evaluated.

Chapter 1 is a general introduction and describes the aim and outline of the thesis.

Chapter 2 describes the smoothness of the stromal bed and the accuracy of the predicted depth after femtosecond laser preparation of posterior lamellar disc (PLD). Nineteen human donor eyes unsuitable for corneal transplantation were used for histologic evaluation. The femtosecond laser is able to prepare a deep horizontal cut in a standardized method with a relatively smooth surface, without extensive adjacent tissue damage.

In Chapter $\mathbf{3}$ we evaluate the effect of the femtosecond laser on endothelial cell viability. There was no significant effect of laser frequency on endothelial cell loss. The dissection of a PLD from the anterior cornea using a blunt dissection technique does not result in significant endothelial cell loss.

Chapter 4 describes a case report of the first patient with pseudophakic bullous keratoplasty treated by FS-DSEK. After 4 months, the PLD was clear and the induced astigmatism was 2.1 diopters (D), demonstrating a functional corneal endothelial layer.

In Chapter $\mathbf{5}$ we describe the preliminary visual results and endothelial cell density of FS-DSEK in 20 eyes with endothelial cell dysfunction. In our study, at the 6-month follow-up, $50 \%$ of patients with a normal visual potential showed a significant improvement of their best spectacle-corrected visual acuity (BSCVA). We also found a hyperopic shift, which can be explained by the meniscus shape of the PLD, which was comparable with previously reported results.

Chapter 6 and Chapter 7 discuss the results of our randomized multicenter clinical trial comparing FS-DSEK and PK of patients with endothelial cell dysfunction. Chapter 6 reports on the clinical outcomes and incidence of postoperative complications between FS-DSEK versus PK. Eighty eyes of 80 patients were treated. At the 1 year follow-up, the percentage of patients with astigmatism lower than $3 \mathrm{D}$ was higher in the FS-DSEK group compared to the PK group. After 1 year, the mean BSCVA was better after PK compared to FS-DSEK, but BSCVA gain was not significantly different between the two groups. Endothelial cell loss is significantly higher in the FS-DSEK group (65\%) compared to the PK group (23\%), which may be explained by the several surgical steps in the FS-DSEK procedure. The most common postoperative complication in the FS-DSEK group was graft dislocation and in the PK group this was wound healing related problems. Chapter 7 reports on the results of straylight and contrast sensitivity between FS-DSEK versus PK. We found a significant improvement of straylight and contrast sensitivity after both FS-DSEK and PK. 
Chapter 8 evaluates the cost-effectiveness of PK, FS-DSEK and Descemet stripping automated endothelial keratoplasty (DSAEK). Data of 118 patients with corneal endothelial cell dysfunction were analyzed. To evaluate cost-effectiveness, incremental cost-effectiveness ratios (ICERs) were calculated. The primary ICER was the incremental costs per clinically improved patient, defined as a patient with a combined effectiveness of both a clinically improved BSCVA (defined as an improvement of $\geq 2$ lines) and a clinically acceptable refractive astigmatism (defined as $\leq 3.0 \mathrm{D}$ ). In our results, FS-DSEK was less effective and more costly compared to both PK and DSAEK. On the other hand, DSAEK was more costly but also more effective compared to PK.

Chapter 9 describes the results of a case series of 5 eyes undergoing femtosecond laser-assisted inverted mushroom keratoplasty. The femtosecond laser was used to create a 'top-hat' configuration in the donor and recipient cornea. After 1 year, all corneal grafts were clear and showed an excellent adaptation of the lamellar donor and recipient wound surfaces. Fast wound healing was noted in 2 cases in which all sutures were removed at 19 and 21 weeks.

Chapter 10 and Chapter 11 describe the results of our randomized multicenter clinical trial comparing DALK and PK in eyes with corneal stromal pathology without endothelial disease. Chapter 10 reports on endothelial cell loss and visual outcome after DALK versus PK. One year after DALK, we found a significantly lower endothelial cell loss in comparison to PK, whereas BSCVA was comparable between the two groups. Descemet's membrane perforation remains a major challenge in DALK, necessitating further surgical improvements. In Chapter 11 we discuss the results of straylight and contrast sensitivity after DALK and PK. At the 1-year follow-up, straylight was not significantly different after DALK compared to PK. In addition, there was a significant improvement in straylight and contrast sensitivity 1 year after DALK and PK.

Chapter 12 evaluates the cost-effectiveness of DALK versus PK in our randomized multicenter clinical trial. The main outcome measures were incremental cost-effectiveness ratios per clinically improved patient on the 25-item National Eye Institute Visual Functioning Questionnaire and per patient with endothelial cell loss of maximally $20 \%$ within the first year. In our study, we found that DALK is more costly and more effective as compared with PK. Results on the 25-item National Eye Institute Visual Functioning Questionnaire were in favor of DALK, and the endothelial cell loss in DALK patients remained stable after 6 months, whereas endothelial cell loss in PK patients continued. 



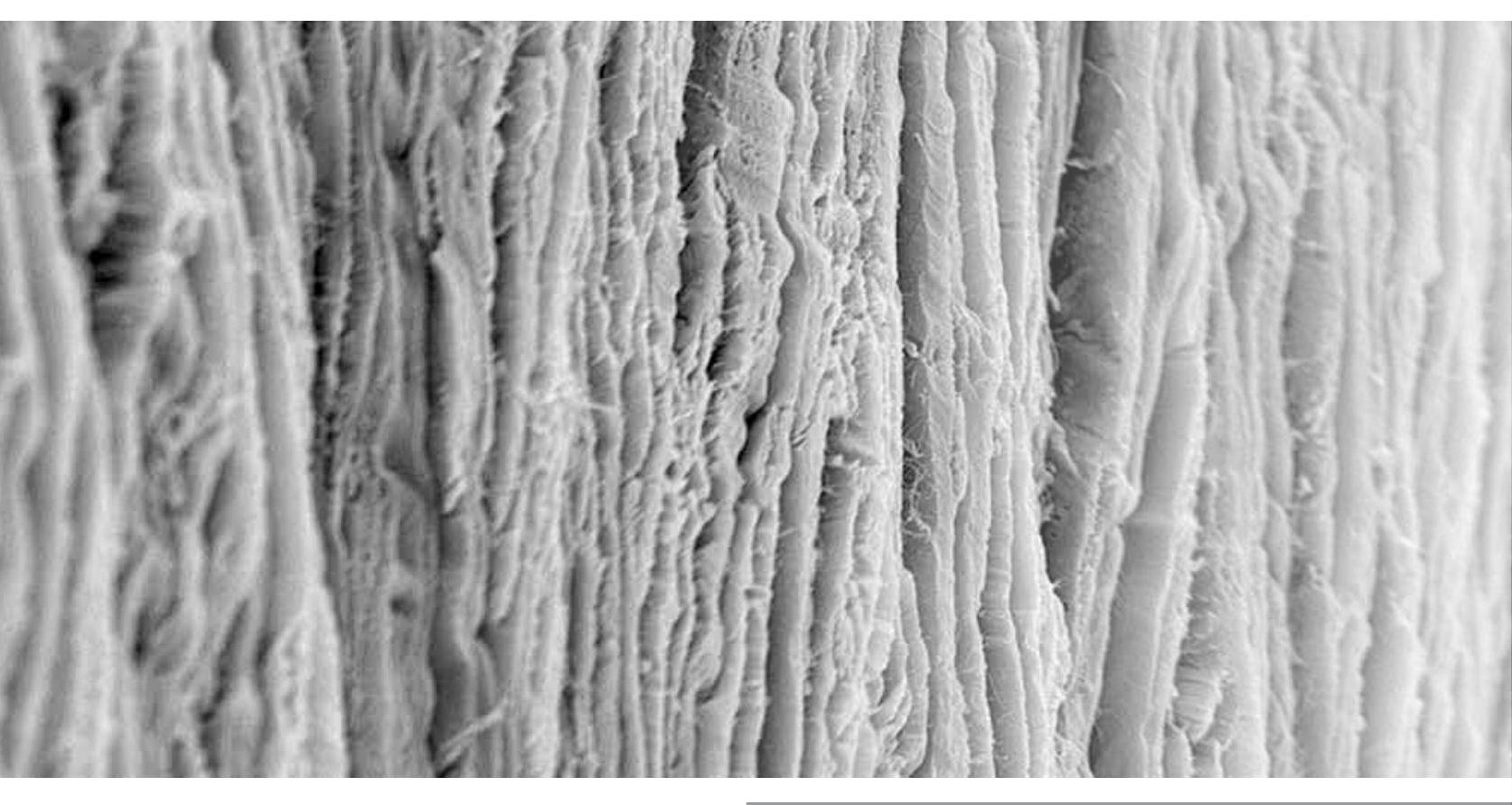



Dit proefschrift beschrijft de klinische resultaten van femtosecond laser Descemet's stripping endothelial keratoplastiek (FS-DSEK) en diep anterieure lamellaire keratoplastiek (DALK) versus perforerende keratoplastiek (PK) en evalueert de rol van de femtosecond laser bij corneatransplantaties.

Hoofdstuk 1 is een algemene introductie en beschrijft de doelstellingen van dit proefschrift.

Hoofdstuk 2 beschrijft de gladheid, nauwkeurigheid en de voorspellende diepte van het stromale bed van de posterieure lamel zoals geprepareerd met de femtosecond laser. Negentien humane donor ogen die ongeschikt waren voor transplantatie werden gebruikt voor histologische evaluatie. Met de femtosecond laser is het mogelijk op gestandaardiseerde wijze een diepe horizontale snijvlak te creëren met een relatief glad oppervlak en weinig weefsel schade.

In hoofdstuk 3 wordt het effect van de femtosecond laser op endotheelcel kwaliteit geëvalueerd. Er was geen significant effect van de laser frequentie op endotheelcel verlies. Het los prepareren van de posterieure lamel met een canule gaf geen significant endotheelcel verlies.

Hoofdstuk 4 beschrijft de eerste casus van een patiënt met pseudofake bulleuze keratopathie die behandeld werd met FS-DSEK. Vier maanden postoperatief was de posterieure lamel helder en het geïnduceerde astigmatisme was 2.1 dioptrie (D), en was er een functionerende endotheelcel laag.

In hoofdstuk 5 beschrijven we de voorlopige visuele resultaten en de endotheelcel dichtheid na FS-DSEK bij 20 ogen met een endotheel dysfunctie. Zes maanden postoperatief vertoonden $50 \%$ van de patiënten (zonder andere visusbeperkende factoren) een verbetering van hun best gecorrigeerde visus (BSCVA). Postoperatief was er sprake van een hyperope verandering, verklaard door de meniscus-vorm van de posterieure lamel.

Hoofdstuk 6 en hoofdstuk 7 bespreken de resultaten van onze gerandomiseerde multicenter studie van FS-DSEK en PK bij patiënten met een endotheel dysfunctie. Hoofdstuk 6 rapporteert de klinische resultaten en incidentie van postoperatieve complicaties na FS-DSEK en PK. Tachtig ogen van 80 patiënten werden behandeld. Een jaar postoperatief was het percentage patiënten met astigmatisme $<3$ D hoger in de FSDSEK groep dan in de PK groep. De gemiddelde BSCVA was beter in de PK groep, maar de visuswinst was niet significant verschillend tussen beide groepen. Endotheelcel verlies was significant hoger in de FS-DSEK groep (65\%) in vergelijking met de PK groep (23\%). Dit verschil wordt mogelijk verklaard door de verschillende chirurgische stappen bij de FS-DSEK procedure. De meest voorkomende postoperatieve complicatie in de FS-DSEK groep was een afliggende donor lamel; in de PK groep waren dit wond gerelateerde problemen. Hoofdstuk $\mathbf{7}$ bespreekt de resultaten van strooilicht en contrastsensitiviteit na FS-DSEK versus PK. Wij vonden een significante verbetering van strooilicht en contrast sensitiviteit na zowel FS-DSEK als PK. 
Hoofdstuk 8 evalueert de kosteneffectiviteit van PK, FS-DSEK en Descemet's stripping automated endothelial keratoplastiek (DSAEK). De data van 118 patiënten met endotheel dysfunctie werden geanalyseerd. Om de kosteneffectiviteit te evalueren, werden incrementele kosteneffectiviteitsratios (ICERs) berekend. De primaire ICER was de incrementele kosten per klinische verbetering bij de patiënt; dit betekent een gecombineerde effectiviteit van klinische verbetering van BSCVA (verbetering van $>1$ Snellen visus regel) en acceptabele refractie astigmatisme ( $\leq 3.0 \mathrm{D})$. In onze resultaten was FS-DSEK minder effectief en meer kostbaar in vergelijking met PK en DSAEK. DSAEK is effectiever in vergelijking met PK, maar leidt ook tot hogere kosten.

Hoofdstuk 9 beschrijft de resultaten van 5 ogen die met femtosecond laser-assisted inverted mushroom keratoplastiek waren behandeld. Met behulp van de femtosecond laser werd een 'top-hat' configuratie gecreëerd in de donor en recipiënt cornea. Een jaar postoperatief waren alle donor corneae helder, met een uitstekende adaptatie tussen de donor en recipiënt cornea. Bij 2 ogen zagen we een snelle wondgenezing optreden waardoor alle hechtingen al na 19 en 21 weken verwijderd konden worden.

Hoofdstuk 10 en 11 bespreken de resultaten van een gerandomiseerde multicenter studie van DALK versus PK bij ogen met cornea pathologie zonder endotheel dysfunctie. Hoofdstuk 10 rapporteert het endotheelcel verlies en de visuele uitkomsten na DALK versus PK. Een jaar na DALK, zagen we significant minder endotheelcelverlies na DALK in vergelijking met PK. De BSCVA was vergelijkbaar tussen de twee groepen. Perforatie van het Descemet membraan blijft een belangrijke uitdaging bij DALK en vergt nog verdere chirurgische verbeteringen. In Hoofdstuk 11 werden de resultaten van strooilicht en contrastsensitiviteit na DALK en PK besproken. Bij de 1 jaar follow-up zagen we geen significant verschil in strooilicht tussen DALK en PK. In beide groepen zagen we een significante verbetering van strooilicht en contrast sensitiviteit na 1 jaar bij zowel DALK als PK.

Hoofdstuk 12 evalueert de kosteneffectiviteit van DALK versus PK in onze gerandomiseerde multicenter studie. De belangrijkste uitkomst maten van ICER waren verbetering per klinische patiënt gemeten met behulp van de 25-item item National Eye Institute Visual Functioning Questionnaire en per patiënt met maximale endotheelcelverlies van $20 \%$ in het eerste postoperatieve jaar. In onze studie leverde DALK meer kosten op, maar was het wel effectiever dan PK. De scores van de 25-item item National Eye Institute Visual Functioning Questionnaire waren beter in de DALK groep en het endotheelcel verlies in de DALK groep bleef na 6 maanden stabiel, terwijl in de PK groep er nog sprake was van toenemend endotheelcel verlies. 



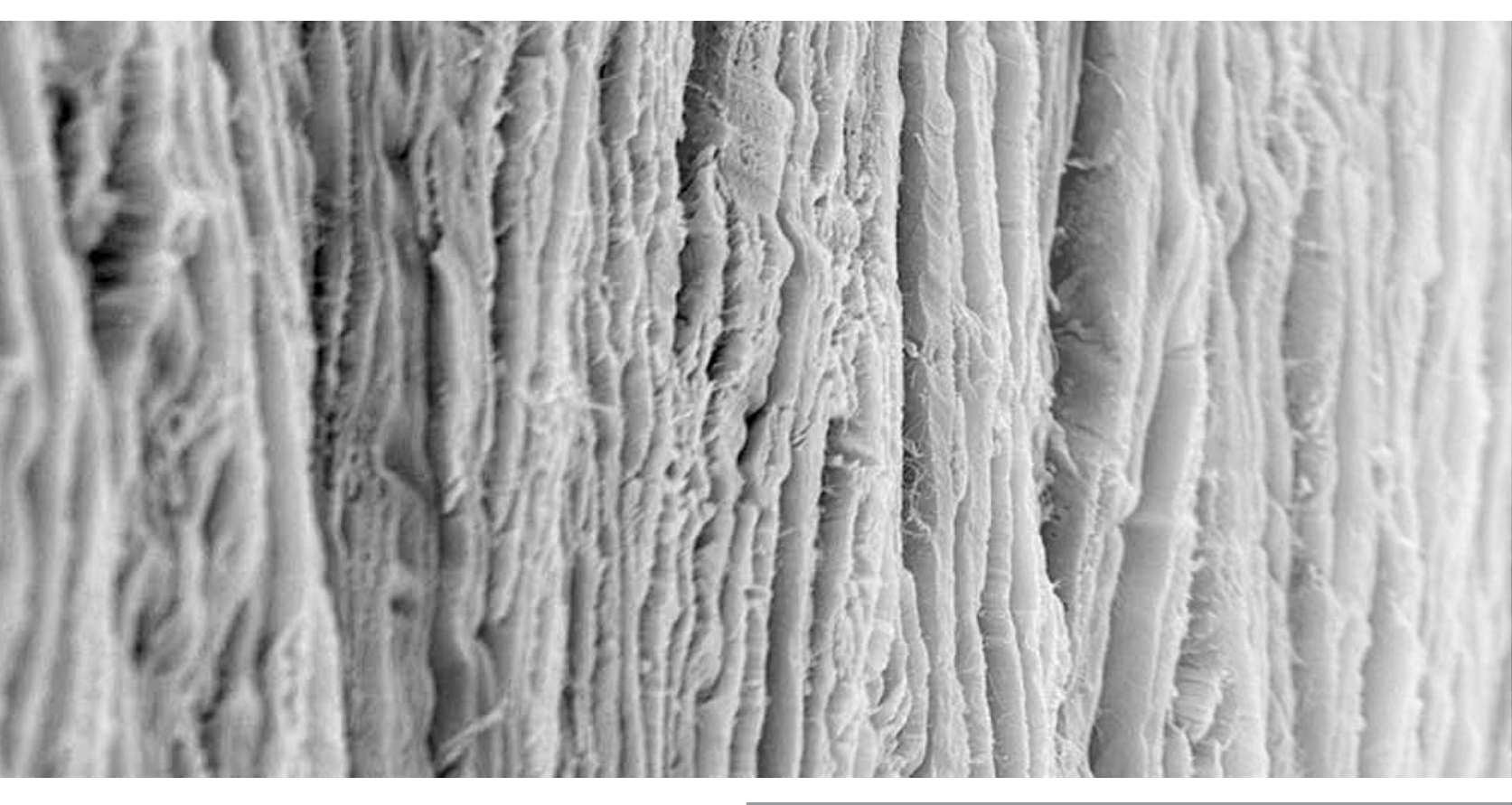



Het avontuur van mijn promotieonderzoek heeft uiteindelijk geleid tot dit proefschrift. Bij de voltooiing van dit proefschrift waren veel mensen direct en indirect betrokken geweest. Een aantal mensen zou ik graag persoonlijk hier willen bedanken.

Allereerst wil ik mijn promotor Prof. dr. R.M.M.A. Nuijts bedanken. Beste Rudy, tijdens mijn sollicitatie stelde je mij voornamelijk vragen met betrekking tot onderzoek doen. Ik kreeg uiteindelijk een zeer interessant project van je aangeboden. Ik verbaasde me weer telkens hoe veel energie je hebt. Na elke congres kwam je weer met nieuwe ideeën en plannen. Ik wil je bedanken voor de negen jaar begeleiding die ik van je heb mogen ontvangen. Verder wil ik je ook bedanken dat je mij hebt willen opleiden als cornea specialist. Ik heb daarom ook een stelling ter ere aan jou gewijd. Je bent een goede leermeester geweest, die ik nooit zal vergeten.

Mijn co-promotor Jan Schouten; beste Jan, ik wil je bedanken voor jouw hulp en kritische blik op onze statische analyse in dit proefschrift. Het was in het begin soms een beetje lastig om je te volgen, je bent zo bevlogen in de statistiek. Het lukte je altijd de reviewers van artikelen te overtuigen met hun lastige vragen, daar heb ik altijd bewondering voor gehad.

De leden van de beoordelingscommissie, Prof. dr. H.W.M. Steinbusch, Prof. dr. S.M. Imhof, Prof. dr. G.P.M. Luyten, Prof. dr. P.M. Steijlen en Prof. dr. M.G.J. Tilanus wil ik bedanken voor hun bereidheid om mijn proefschrift te lezen en beoordelen.

Het voormalig hoofd van de Universiteitskliniek voor Oogheelkunde Maastricht, Prof. dr. Fred Hendrikse, mijn eerste opleider, die mij heeft aangenomen om te starten met mijn promotieonderzoek en vervolgens voor de opleiding tot oogarts. Bedankt voor uw vertrouwen in mij.

Prof. dr. Carroll Webers, mijn tweede opleider, en Astrid Hacking wil ik graag bedanken voor de faciliteiten en steun die ik heb gekregen tijdens mijn opleiding en cornea fellowship. Alle medewerkers van de afdeling Oogheelkunde in het Maastricht Universiteit Medisch centrum wil ik bedanken voor alle jaren van steun en hulp.

Alle onderzoekers (Aukje, Andrea, Rob, Annelie, Lukas, Muriël, Paul, Nienke, Margriet, Mari, Elton, Frenne, Frank) van de afgelopen jaren wil ik bedanken voor de serieuze discussies over onze onderzoeken, maar ook voor de gezellige lunch meetings, tea-breaks en happy hours. 
Thea van Weersch, veel dank voor het regelen van alles in St. Annadal en dat ik daar altijd terecht kon met mijn donor ogen voor de studie.

Alle medewerkers van de voormalige Cornea Bank in Amsterdam en in het bijzonder Liesbeth Pels; ik wil jullie graag bedanken voor de medewerking aan de studie. Liesbeth, jij bent altijd bereid geweest met ons mee te denken. Jouw kennis over donor cornea was erg belangrijk voor ons.

I would like to thank Hans Grossniklaus for his contribution to our article and the beautiful histologic photographs.

Ook dank aan mijn andere mede-auteurs, Shin J. Kang, Hans Duimel, Peter Frederik, Jack Cleuntjes, Robert J. van Suylen, Frank van den Biggelaar, Carmen Dirksen, Nayyirih Tahzib en Nienke Visser. Tom van de Berg, bedankt voor de analyse van de strooilicht metingen en Frank ook erg bedankt van de mooie economische berekeningen. Alle oogartsen die hebben deelgenomen aan de "Dutch Lamellar Corneal Transplantation Study": RobertJan Wijdh, Cathrien Eggink, Prof. Riel van Rij, Annemiek Rijneveld, Michel Zaal en Hugo van Cleynenbreugel; veel dank voor jullie bijdrage aan deze belangrijke studie.

Mijn huidige leidinggevende Prof. dr. Gre Luyten. Beste Gre, bedankt voor je steun tijdens het laatste stukje van mijn proefschrift. Ook dank aan iedereen van de afdeling Oogheelkunde van het Leids Universitair Medisch Centrum voor hun leuke ideeën en tips voor mijn proefschrift.

Mijn paranimfen: Pei-Yu en Nayyirih. Pei-Yu, we kennen elkaar al vanaf jongs af aan. We hebben veel samen meegemaakt en op jou kan ik rekenen. Na mijn terugkomst in de Randstad kunnen we weer vaker gezellige dingen doen. Nayyirih, je was een van de eerste personen aan wie Rudy me had voorgesteld. Ik kan me bijna niet voorstellen hoe ik het allemaal zonder jou had moeten doen. Je bent een belangrijke support voor mij geweest, want we weten allebei hoe zwaar het is om onderzoek bij Rudy te doen. Je hebt mij de afgelopen maanden veel geholpen om het laatste stukje van mijn proefschrift af te maken. Ik ben erg dankbaar voor jullie steun !

Tenslotte wil ik mijn ouders en broers bedanken. Bedankt voor jullie steun en vertrouwen in mij. 



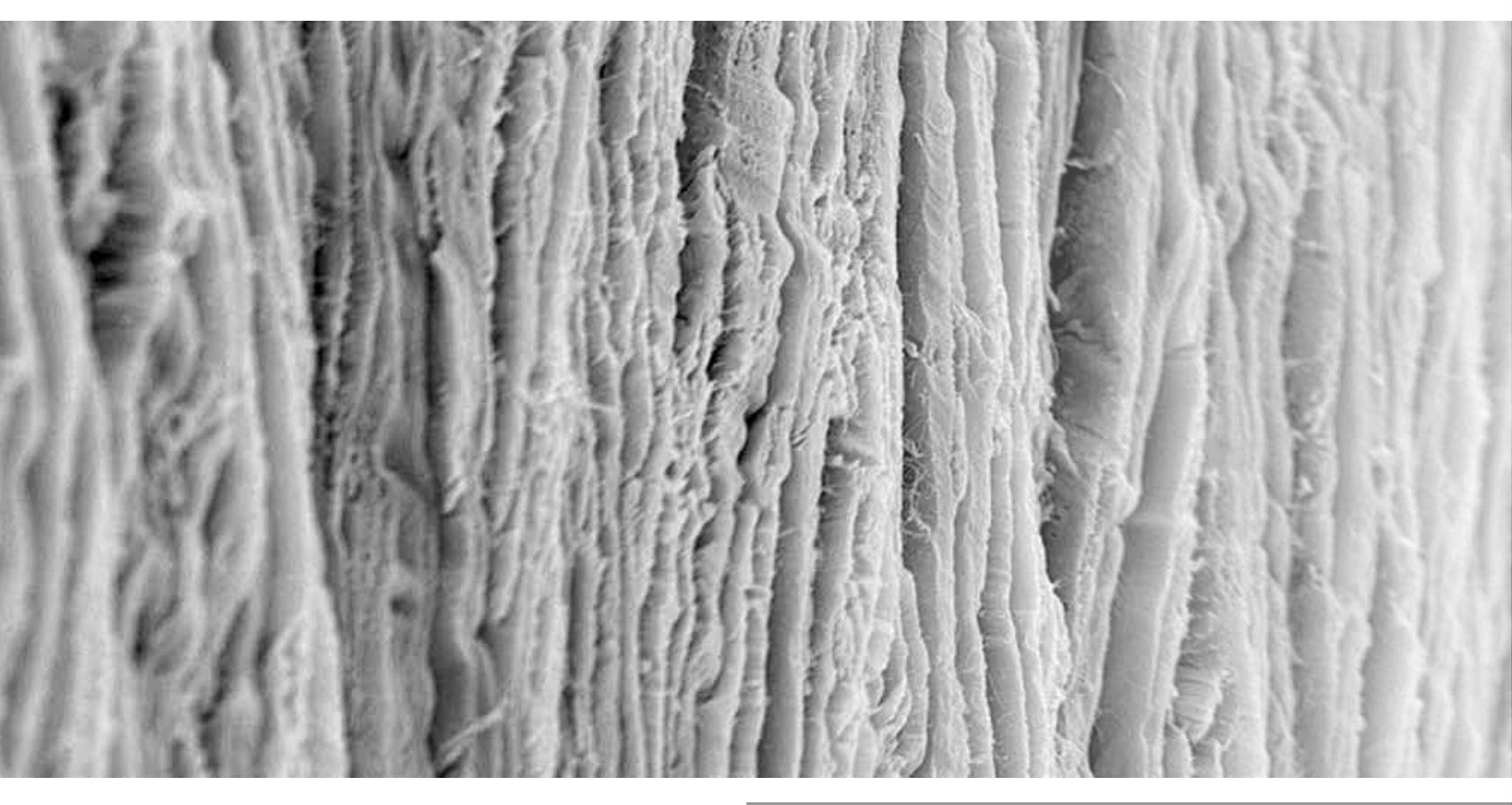


Curriculum Vitae 



\section{CURRICULUM VITAE}

Yanny Ying-Yee Cheng werd geboren op 5 mei 1979 te Rotterdam. Na het behalen van haar Atheneum diploma aan de Christelijke Scholengemeenschap Calvijn te Rotterdam in 1998, startte zij met haar studie geneeskunde aan de Erasmus MC Universiteit in Rotterdam, waar zij op 20 augustus 2004 haar artsendiploma behaalde. In januari 2005 ving haar promotieonderzoek in het Maastricht Universitair Medisch Centrum aan, waarvan de resultaten in dit proefschrift zijn beschreven. Vervolgens startte ze onder leiding van Prof. dr. F. Hendrikse en Prof. dr. C.A.B. Webers in januari 2008 haar opleiding tot oogarts, waarna zij van januari 2013 tot januari 2014 onder leiding van Prof. dr. R.M.M.A. Nuijts als cornea fellow werkte. Gedurende dit cornea fellowship, werkte zij tevens als oogarts in de nevenklinieken in Heerlen en Brunssum. Vanaf 2014 tot juli 2015 verhuisde zij naar Leiden en startte zij als oogarts en corneaspecialist in het Leids Universiteit Medisch Centrum en Bronovo Ziekenhuis te Den Haag. Momenteel werkt zij fulltime in het Leids Universiteit Medisch Centrum. 



\section{LIST OF PUBLICATIONS}

Bhagwandien, A.C., Cheng, Y.Y., Wolfs, R.C., van Meurs, J.C., Luyten, G.P.M. Relationship between retinal detachment and biometry in 4262 cataractous eyes. Ophthalmology, 2006;113(4):643-49.

Tahzib NG, Cheng YY, Nuijts RM. Three-year follow-up analysis of Artisan toric lens implantation for correction of postkeratoplasty ametropia in phakic and pseudophakic eyes. Ophthalmology. 2006 Jun;113(6):976-84.

Cheng, Y.Y.Y, Pels, E., Nuijts,R.M.M.A. Femtosecond laser assisted Descemet Stripping Endothelial Keratoplasty. Journal of Cataract and Refractive Surgery, 2007; 33 (1):152155.

Cheng, Y.Y.Y., Pels, E., Cleutjens, J.P.M., van Suylen, R.J., Hendrikse, F., Nuijts, R.M.M.A. Corneal endothelial viability after femtosecond laser preparation of posterior lamellar discs for Descemet-stripping endothelial keratoplasty. Cornea, 2007;26(9):1118-22.

de Vries NE, Franssen L, Webers CA, Tahzib NG, Cheng YY, Hendrikse F, Tjia KF, van den Berg TJ, Nuijts RM. Intraocular straylight after implantation of the multifocal AcrySof ReSTOR SA60D3 diffractive intraocular lens. J Cataract Refract Surg. 2008 Jun;34(6):95762.

Cheng, Y.Y.Y., Tahzib, N.G., van Rij, G., van Cleynenbreugel, H., Pels, E., Hendrikse, F., Nuijts, R.M.M.A. Femtosecond Laser-assisted Inverted Mushroom Keratoplasty. Cornea, Cornea. 2008 Jul;27(6):679-85.

de Vries NE, Webers CA, Montés-Micó R, Tahzib NG, Cheng YY, de Brabander J, Hendrikse F, Nuijts RM. Long-term follow-up of a multifocal apodized diffractive intraocular lens after cataract surgery. J Cataract Refract Surg. 2008 Sep;34(9):1476-82.

Cheng YY, Hendrikse F, Pels E, Wijdh RJ, van Cleynenbreugel H, Eggink CA, van Rij G, Rijneveld WJ, Nuijts RM. Preliminary results of femtosecond laser-assisted descemet stripping endothelial keratoplasty. Arch Ophthalmol. 2008 Oct;126(10):1351-6.

Cheng YY, Kang SJ, Grossniklaus HE, Pels E, Duimel HJ, Frederik PM, Hendrikse F, Nuijts RM. Histologic evaluation of human posterior lamellar discs for femtosecond laser Descemet's stripping endothelial keratoplasty. Cornea. 2009 Jan;28(1):73-9. 
Cheng YY, Schouten JS, Tahzib NG, Wijdh RJ, Pels E, van Cleynenbreugel H, Eggink CA, Rijneveld WJ, Nuijts RM. Efficacy and safety of femtosecond laser-assisted corneal endothelial keratoplasty: a randomized multicenter clinical trial. Transplantation. 2009 Dec 15;88(11):1294-302.

de Vries NE, Webers CA, Verbakel F, de Brabander J, Berendschot TT, Cheng YY, Doors $M$, Nuijts RM. Visual outcome and patient satisfaction after multifocal intraocular lens implantation: aspheric versus spherical design. J Cataract Refract Surg. 2010 Nov;36(11):1897-904.

Cheng YY, Visser N, Schouten JS, Wijdh RJ, Pels E, van Cleynenbreugel H, Eggink CA, Zaal MJ, Rijneveld WJ, Nuijts RM. Endothelial Cell Loss and Visual Outcome of Deep Anterior Lamellar Keratoplasty versus Penetrating Keratoplasty: A Randomized Multicenter Clinical Trial. Ophthalmology. 2011 Feb;118(2):302-9.

Van den Biggelaar FJ, Cheng YY, Nuijts RM, Schouten JS, Wijdh RJ, Pels E, van Cleynenbreugel H, Eggink CA, Zaal MJ, Rijneveld WJ, Dirksen CD. Economic evaluation of deep anterior lamellar keratoplasty versus penetrating keratoplasty in The Netherlands. Am J Ophthalmol. 2011 Mar;151(3):449-59.e2.

Cheng YY, van den Berg TJ, Schouten JS, Pels E, Wijdh RJ, van Cleynenbreugel H, Eggink CA, Rijneveld WJ, Nuijts RM. Quality of vision after femtosecond laser-assisted descemet stripping endothelial keratoplasty and penetrating keratoplasty: a randomized, multicenter clinical trial. Am J Ophthalmol. 2011 Oct;152(4):556-566.e1.

van den Biggelaar FJ, Cheng YY, Nuijts RM. Deep anterior lamellar keratoplasty. Ophthalmology. 2011 Nov;118(11):2305-6; author reply 2307.

van den Biggelaar FJ, Cheng YY, Nuijts RM, Schouten JS, Wijdh RJ, Pels E, van Cleynenbreugel H, Eggink CA, Rijneveld WJ, Dirksen CD. Economic evaluation of endothelial keratoplasty techniques and penetrating keratoplasty in the Netherlands. Am J Ophthalmol. 2012 Aug;154(2):272-81.e2

Dickman MM, Cheng YY, Berendschot TT, van den Biggelaar FJ, Nuijts RM. Effects of graft thickness and asymmetry on visual gain and aberrations after descemet stripping automated endothelial keratoplasty. JAMA Ophthalmol. 2013 Jun 1;131(6):737-44. 

